\title{
MEASUREMENT OF ROOM CONDITIONS AND RESPONSE OF SPRINKLERS AND SMOKE DETECTORS DURING A SIMULATED TWO-BED HOSPITAL PATIENT ROOM FIRE
}

Kathy A. Notarianni

Building and Fire Research Laboratory

Gaithersburg, Maryland 20899

$-Q C$

100

.056

110.5240

1993 



\section{MEASUREMENT OF ROOM CONDITIONS AND RESPONSE OF SPRINKLERS AND SMOKE DETECTORS DURING A SIMULATED TWO-BED HOSPITAL PATIENT ROOM FIRE}

Kathy A. Notarianni

July 1993

Building and Fire Research Laboratory

National Institute of Standards and Technology

Gaithersburg, MD 20899

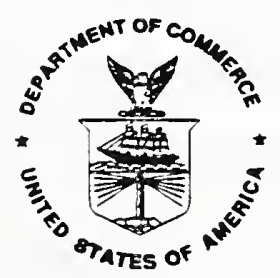

U.S. Department of Commerce

Ronald H. Brown, Secretary

Technology Administration

Mary L. Good, Under Secretary for Technology

National Institute of Standards and Technology

Arati Prabhakar, Director 


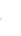


List of Figures

Abstract

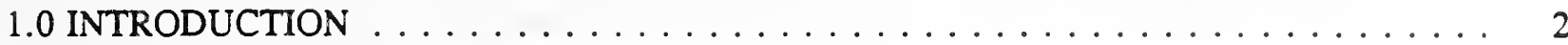

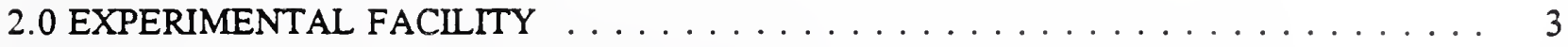

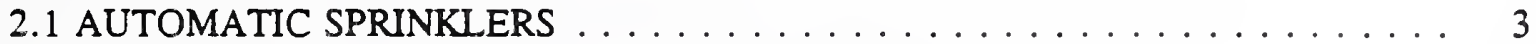

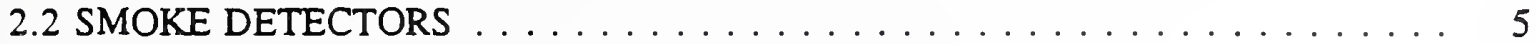

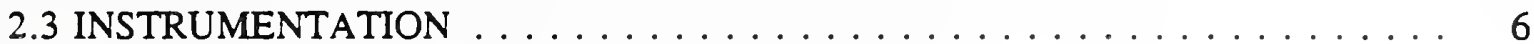

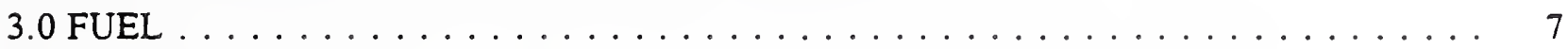

4.0 TEST SCENARIOS $\ldots \ldots \ldots \ldots \ldots \ldots \ldots \ldots \ldots \ldots \ldots \ldots$

5.0 TEST DESCRIPTIONS $\ldots \ldots \ldots \ldots \ldots \ldots \ldots \ldots \ldots \ldots$

5.1 CLOSED DOOR TEST $\ldots \ldots \ldots \ldots \ldots \ldots \ldots$

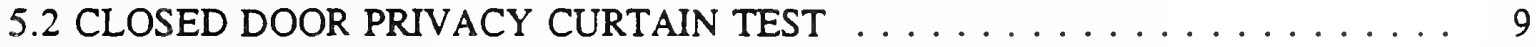

5.3 OPEN DOOR PRIVACY CURTAIN TEST $\ldots \ldots \ldots \ldots \ldots \ldots \ldots$

5.4 CLOSED DOOR SHIELDED FIRE TEST $\ldots \ldots \ldots \ldots \ldots \ldots \ldots$

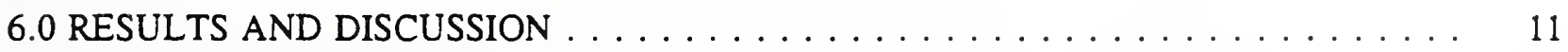

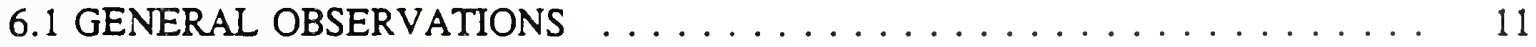

6.2 TIME AND TEMPERATURE AT TIME OF ACTIVATION $\ldots \ldots \ldots \ldots \ldots$

6.2 .1 QUICK RESPONSE SPRINKLERS . . . . . . . . . . . . . . . . . 12

6.2 .2 CONCEALED QUICK RESPONSE SPRINKLERS . . . . . . . . . 13

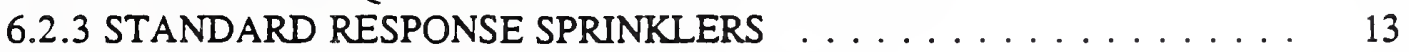

6.2 .4 IONIZATION DETECTORS . . . . . . . . . . . . . . . . . . 14

6.2.5 PHOTOELECTRIC DETECTORS/SENSORS . . . . . . . . . . . . . 14

6.3 GAS CONCENTRATIONS . . . . . . . . . . . . . . . . . . . . . 14

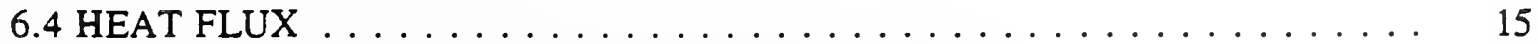

6.5 TENABILITY, HAZARD I, AND THE N-GAS MODEL . . . . . . . . . . 15

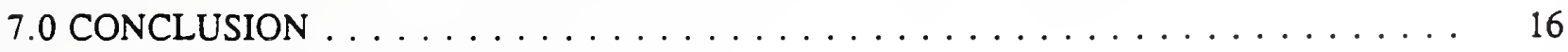

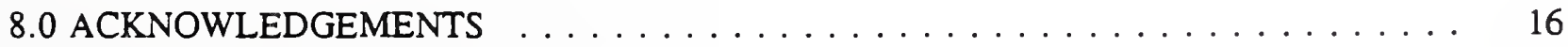

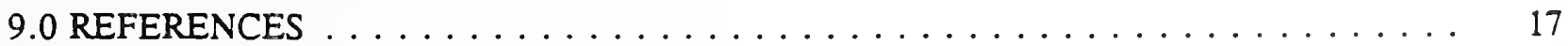

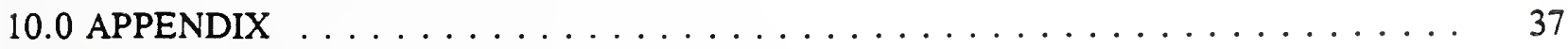




\section{List of Tables}

Table 1. Activation times, closed door test ........................ Page

Table 2. Activation times, closed door privacy curtain test . . . . . . . . . . . . . . . . . . 19

Table 3. Activation times, open door privacy curtain test . . . . . . . . . . . . . . . . 20

Table 4. Activation times, closed door shielded fire test . . . . . . . . . . . . . . . 21

Table 5. Comparison of response time: concealed quick response, quick response, and standard

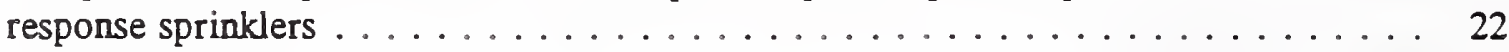

Table 6. Summary of test results: Gas sampling and heat flux data . . . . . . . . . . . . 23 
List of Figures

Figure 1. Plan view of simulated hospital patient room and corridor . . . . . . . . . . . . 24

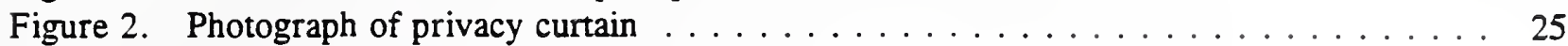

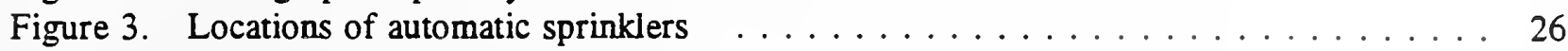

Figure 4. Locations of smoke detectors . . . . . . . . . . . . . . . . . . . . 27

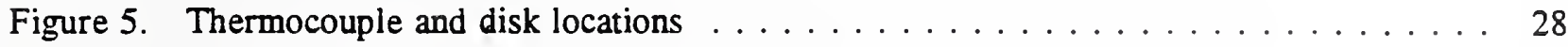

Figure 6. Gas sampling and heat flux meter locations . . . . . . . . . . . . . . . . . . . . . . . 29

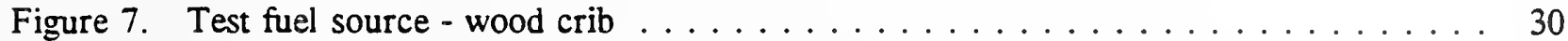

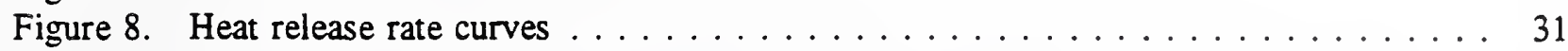

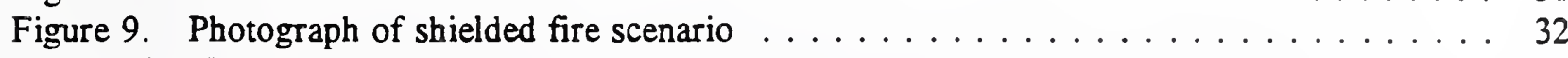

Figure 10. Temperatures in room, closed door test $\ldots \ldots \ldots \ldots \ldots \ldots$

Figure 11. Temperatures in room, closed door privacy curtain test . . . . . . . . . . . . . . 34

Figure 12. Temperatures in room, open door privacy curtain test $\ldots \ldots \ldots \ldots \ldots$

Figure 13. Temperatures in room, closed door shielded fire test . . . . . . . . . . . . . . 36 
' 


\title{
MEASUREMENT OF ROOM CONDITIONS AND RESPONSE OF SPRINKLERS AND SMOKE DETECTORS DURING A SIMULATED TWO-BED HOSPITAL PATIENT ROOM FIRE
}

\author{
Kathy A. Notarianni \\ Building and Fire Research Laboratory \\ National Institute of Standards and Technology \\ Gaithersburg, MD 20899
}

\begin{abstract}
A series of experiments are reported in which a wood crib was burned within a simulated two bed hospital patient room in order to measure the activation times of various types of quick and standard response sprinklers and ionization and photoelectric smoke detectors at several locations in the room simulating multiple options for protection of the space. Gas and surface temperatures, heat flux, carbon dioxide, carbon monoxide, and oxygen concentrations were continuously measured in order to access the tenability of the room. Of the parameters measured, temperature was the best indicator of the tenability of the space. Temperature at time of activation of the quick response sprinklers was at or below $77{ }^{\circ} \mathrm{C}$ $\left(171{ }^{\circ} \mathrm{F}\right)$ at the five foot level and at or below $48^{\circ} \mathrm{C}\left(118^{\circ} \mathrm{F}\right)$ at the three foot level between the patient beds, in all tests with the exception of the shielded fire scenario where temperatures at the five and three foot levels reached $111^{\circ} \mathrm{C}\left(232^{\circ} \mathrm{F}\right)$, and $78^{\circ} \mathrm{C}\left(172{ }^{\circ} \mathrm{F}\right)$ respectively. An initial detector activation was received between 232-377 seconds prior to activation of the first sprinkler, and 552-722 seconds prior to activation of the QR-EC sidewall sprinkler.
\end{abstract}

Key words: Sprinklers; fire research; fire tests; sprinkler response; smoke detectors; hospital fires; life safety 


\subsection{INTRODUCTION}

Advances in sprinkler technology have led to the development of quick response (QR) sprinklers which, due to their lower thermal inertia, actuate earlier in the fire growth than standard response sprinklers of the same temperature rating. By initiating earlier fire suppression, quick response sprinklers, when properly applied, can reduce the quantity of heat and combustion products generated, thus offering the potential for providing both life safety and property protection.

The National Institute of Standards and Technology (NIST), formally the National Bureau of Standards, performed studies of sprinklered patient room fires in the late 1970's as part of a five year life safety/fire safety research program. $[1,2]$ Some of the experiments simulated a link with lower thermal inertia than existed commercially for sprinklers at that time. ${ }^{1}$ The results showed that the amount of smoke and other products of combustion was greatly reduced by means of the low thermal inertia sprinkler. More recent studies utilized quick response sprinklers in office configurations [3] and hotels.[4] These experiments showed that quick response sprinklers can substantially improve life safety and property protection in these occupancies as well.

In occupancies such as hospitals, where the patient may be non-ambulatory, rapid fire detection and suppression may be able to suppress fire growth sufficiently to maintain survivable conditions even in the room in which the fire originated. Options for protection of a hospital patient room include the use of conventional sprinklers, quick response sprinklers, smoke detectors, or a combination of sprinklers and smoke detectors. Requirements for installation of sprinklers and/or smoke detectors in these facilities are being considered with in the National Fire Protection Association (NFPA) standards development process. The Life Safety Code, NFPA 101 [5], currently requires automatic sprinkler protection utilizing quick response sprinklers for patient sleeping rooms of new health care occupancies. Automatic sprinkler protection is also required in some existing health care occupancies depending of the type of building construction and the number of floors in the building. In these cases, either standard or quick response sprinklers are acceptable. [5]

The National Institutes of Health (NIH) has expressed a need to develop criteria which ensure adequate, cost effective fire protection for hospital patient rooms. The Building and Fire Research Laboratory (BFRL) of NIST under the sponsorship of NIH conducted a series of experiments in a full-scale simulated two-bed hospital patient room. The experiments provided information for evaluating the effectiveness of automatic sprinklers and smoke detectors in response to a nominally $60 \mathrm{~kW}$ flaming fire. Smoldering fires were not included in this study, as these have been addressed in previous work.[6] A data bank of measured fire conditions and activation times of various automatic sprinklers and smoke detectors at several locations within the room was generated. The tests addressed the type and location of sprinkler heads in the hospital patient room, demonstrated the effect of privacy curtains around the beds, the effect of the door opening position, and the effect of a shielded fire.

${ }^{1}$ To simulate the rapid response, the gas temperature near the sprinkler was continuously monitored during the fire test. The sprinkler valve was opened at $10 \mathrm{~s}$ after the time the center ceiling gas temperature reached the operating temperature of the sprinkler. 
This research effort was expanded in scope beyond the original study of sprinkler response through the cooperation of Simplex Time Recorder Co. who, on behalf of the detector industry, provided for the measured smoke detector performance in these hospital patient room fires.

\subsection{EXPERIMENTAL, FACILITY}

A typical two-bed hospital patient room at the National Institutes of Health (NIH) hospital was selected with the assistance of the NIH fire protection staff as a model for this study. The simulated room constructed at NIST maintained the major features and dimensions of the NIH room. The simulated room included the primary patient area, as well as an entrance way and a bathroom.

A plan view of the room and corridor is shown in figure 1 . The room and corridor were fabricated using $92 \mathrm{~mm}$ (3-5/8 in) 25 gauge steel wall studs, and $152 \mathrm{~mm}(6 \mathrm{in}) 25$ gauge steel ceiling joists. The walls and ceiling were $16 \mathrm{~mm}$ ( $5 / 8 \mathrm{in})$ fire rated drywall, covered with a layer of $13 \mathrm{~mm}(1 / 2 \mathrm{in})$ noncombustible calcium silicate board, in place of the paper-covered gypsum board used in the hospital. This fully non-combustible board allowed the use of the simulated patient room for repeated fire tests.

An opening in the ceiling $1.03 \mathrm{~m} \mathrm{(40} \mathrm{1/2} \mathrm{in)} \mathrm{wide} \mathrm{at} \mathrm{the} \mathrm{north} \mathrm{end} \mathrm{of} \mathrm{the} \mathrm{corridor} \mathrm{was} \mathrm{provided} \mathrm{to} \mathrm{allow}$ smoke and products of combustion to vent to a plenum. The plenum volume was sufficient to prevent buildup of combustion products in the corridor outside the patient room door, thereby simulating a much longer corridor than constructed.

A $47.0 \mathrm{l} / \mathrm{s}(100 \mathrm{~cm})$ ceiling exhaust fan was installed in the bathroom as shown in figure 1. Fresh air was drawn through a $0.15 \mathrm{~m}$ (6 in) diameter pipe penetrating the west wall of the patient room. The air in-flow was diffused by a $0.28 \mathrm{~m}$ (11 in) high $\times 0.24 \mathrm{~m}(9-1 / 2 \mathrm{in})$ wide flat plate downstream of the pipe outlet, $9.5 \mathrm{~mm}$ ( $3 / 8 \mathrm{in})$ from the wall. Patient beds were simulated using bed frames provided by NIH with a $13 \mathrm{~mm}$ (1/2 in) non-combustible calcium silicate board used in place of mattress and bedding.

A privacy curtain was installed in the primary patient area of the hospital room midway between the two patient beds. A picture of the privacy curtain is shown in figure 2. The privacy curtain was made of a woven polyester, and was a solid fabric curtain with $48 \mathrm{~cm}$ (19 in) of mesh at the top. Each mesh rectangle measured $0.95 \times 0.6 \mathrm{~cm}(3 / 8 \times 1 / 4 \mathrm{in})$ The privacy curtain was hung from a metal track attached to the ceiling using plastic hooks. This installation is in accordance with NFPA 13, Standard for the Installation of Sprinkler Systems. [7] When the privacy curtain was drawn between the beds, it separated thermocouple tree TR1, and sprinkler S3, the quick response pendent on the ceiling in the center of the room, from the crib fire.

\subsection{Automatic Sprinklers}

In accordance with NFPA 13, there are a number of options for the type and location of sprinklers which may be used in a hospital patient room. Sprinkler location is a function of the type of sprinkler used and the size of the area to be protected. In consultation with the sponsor and an ad hoc advisory committee, six options utilizing quick response sprinklers were selected for inclusion in this study. These options are as follows, with the locations of the automatic sprinklers shown in figure 3 :

1) two quick response pendent sprinklers on the ceiling, one above the foot of each patient bed at locations S2 and S4; 
2) one quick response extended coverage (QR-EC) pendent sprinkler on the ceiling in the center of the room at location S3;

3) two quick response sidewall sprinklers on the south wall, one over the head of each patient bed at locations S11 and S12;

4) two quick response sidewall sprinklers on the north wall, one across from the foot of each patient bed at locations S5 and S7;

5) one quick response extended coverage sidewall sprinkler on the east wall furthest from the fire at location S1;

6) two concealed quick response pendent sprinklers on the ceiling, one above the foot of each patient bed, at locations S9 and S10.

In addition. two standard response sprinklers were installed to obtain information about relative sprinkler response times.

1) one standard response pendent sprinkler on the ceiling, above the foot of patient bed \#1, at location S8;

2) one standard response sidewall sprinkler on the north wall, across from the foot of patient bed $\# 2$, at location S6.

All sprinklers were installed in accordance with NFPA 13 and the listing information provided by the manufacturer. Pendent sprinklers were installed so that the distance from the sprinkler deflector to the ceiling was $5 \mathrm{~cm}$ ( 2 in), except for the concealed pendents whose assemblies were mounted flush with the ceiling. All sidewall sprinklers were installed with the deflector $15 \mathrm{~cm}(6 \mathrm{in})$ down from the ceiling and approximately $5 \mathrm{~cm}(2 \mathrm{in})$ out from the wall.

Sprinklers were $1.27 \mathrm{~cm}(1 / 2 \mathrm{in})$ orifice sprinklers with a $\mathrm{K}$ factor of $0.79(\ell / \mathrm{min} / \mathrm{kPa})^{1 / 2}(5.5$ $\left.(\mathrm{gpm} / \mathrm{psi})^{1 / 2}\right)$. All sprinklers utilized frangible glass bulbs for sensing elements and, with the exception of the concealed quick response sprinklers, had operating temperatures of $68^{\circ} \mathrm{C}\left(155^{\circ} \mathrm{F}\right)$. The quick response and extended coverage sprinklers had $2.8 \mathrm{~mm}(0.11 \mathrm{in})$ diameter bulbs with a response time index (RTI) of $39 \mathrm{~m}^{1 / 2} \cdot \mathrm{s}^{1 / 2}\left(70 \mathrm{ft}^{1 / 2} \cdot \mathrm{s}^{1 / 2}\right)$. The standard response sprinklers had $8.0 \mathrm{~mm}(0.31$ in) diameter bulbs with an RTI of $167 \mathrm{~m}^{1 / 2} \cdot \mathrm{s}^{1 / 2}\left(300 \mathrm{ft}^{1 / 2} \cdot \mathrm{s}^{1 / 2}\right)$. The extended coverage sidewall was a QREC sidewall sprinkler equipped with a fast response link and was Underwriters Laboratories (UL) listed as a quick response sprinkler.

Concealed sprinklers were recessed into the ceiling and concealed by a flat cover plate. The model used in the test series has a $57^{\circ} \mathrm{C}\left(135^{\circ} \mathrm{F}\right), 3.0 \mathrm{~mm}$ diameter glass bulb in a standard style sprinkler frame with a drop down deflector. The cover plate is attached to the skirt using $57^{\circ} \mathrm{C}\left(135^{\circ} \mathrm{F}\right)$ temperature solder. When the ceiling temperature rises, the solder holding the cover plate fuses, thus releasing the plate and exposing the sprinkler inside to the hot ceiling flow. The subsequent shattering of the sprinkler bulb opens the waterway and causes the deflector to drop into position to distribute the discharging water. Concealed quick response sprinklers were U.L. listed as quick response sprinklers. The RTI was not available from the manufacturer for these sprinklers. 
The scope of this study did not permit a sufficient number of experiments to fully examine all of the sprinkler installation options. In consultation with the sponsor and an ad hoc advisory committee, it was decided that only the extended coverage sidewall sprinkler on the east wall of the patient room furthest from the fire (location S1) would be connected to the water supply. Gas burner tests previously performed in the room indicated that this was the slowest of the quick response sprinklers to activate, and therefore it provided for the greatest buildup of heat and products of combustion in the room prior to the initiation of fire suppression. In each of the tests, the QR-EC sidewall sprinkler was charged with water. The coverage area for the QR-EC sidewall sprinkler was the entire living area of the hospital patient room, $4.57 \times 3.53 \mathrm{~m}(15 \times 11.5 \mathrm{ft})$. The flow to the sprinkler was set at $1.64 \mathrm{l} / \mathrm{s}(29 \mathrm{gpm})$ at $191 \mathrm{kPa}$ (27.8 psi) as specified by the manufacturer. Flow was manually controlled using a rotameter and a pressure gauge. The activation time for the QR-EC sidewall sprinkler was determined by the observation of the initiation of water flow.

The activation times of the remaining eleven sprinklers, which were not connected to the water supply, were measured using the air pressure drop in the sprinkler supply lines. Each of the sprinklers was individually piped to an air pressure detection switch. The pressure detection switch for each sprinkler was electrically connected to an elapsed time clock. Prior to the start of an experiment, the piping to each of these sprinklers was pressurized with air at $172 \mathrm{kPa}(25 \mathrm{psig})$. During testing, the activation of a sprinkler connected to an air pressure detection switch resulted in the discharge of the air in the piping and operation of the pressure switch, which stopped the elapsed time clock.

Water application densities for the QR-EC sidewall sprinkler were measured at the fire location (on the load cell), using two collection pans with dimensions of 0.36 by $0.36 \mathrm{~m}$ (14 by 14 in). The average water application density in each of the pans was measured with the QR-EC sidewall sprinkler operating at a flow rate of $1.64 \mathrm{l} / \mathrm{s}(29 \mathrm{gpm})$ as specified by the manufacturer. Average water application densities at the fire location were $162 \mathrm{~mm} / \mathrm{s}\left(0.07 \mathrm{gpm} / \mathrm{ft}^{2}\right)$ without the privacy curtain, and $138 \mathrm{~mm} / \mathrm{s}(0.06$ $\mathrm{gpm} / \mathrm{ft}^{2}$ ) with the privacy curtain drawn between the beds.

\subsection{Smoke Detectors}

To protect this room in accordance with NFPA 72E, Standard on Automatic Fire Detectors [8], one smoke detector is needed in the room. This detector could have an ionization or a photoelectric sensor and could be located on the ceiling or on a sidewall. If the detector is installed on the ceiling, there must not be less than $100 \mathrm{~mm}$ ( 4 in) between a sidewall and the near edge. If the detector is installed on a sidewall, there must be between $100 \mathrm{~mm}$ and $300 \mathrm{~mm}$ ( 4 in and $12 \mathrm{in}$ ) from the ceiling to the top of the detector. In consultation with the sponsor and an ad hoc advisory committee, four detector locations were selected for inclusion in this study. These locations are listed below and shown in figure 4 .

A) On the south wall between the beds

B) On the east wall furthest from the fire

C) On the ceiling near the room door

D) On the ceiling in the center of the room

At each of the four locations selected for study, a group of detectors were installed. Each group consisted of an ionization detector, a photoelectric detector, and a photoelectric sensor that produced an analog signal. Installation, calibration and test data from smoke detector groups were provided by Simplex Time Recorder Co., on behalf of the detector industry. Groups A and B were mounted on sidewalls below the ceiling; groups $\mathrm{C}$ and $\mathrm{D}$ were mounted on the ceiling. Odd numbered detectors, D1, 
D3, D5, and D7 were photoelectric detectors with a sensitivity of nominally 8.6 percent per meter (2.6 percent per foot). Even numbered detectors, D2, D4, D6, and D8, were ionization detectors with a sensitivity of nominally 3.3 percent per meter (1 percent per foot). DSA, DSB, DSC, and DSD were the photoelectric sensors with a sensitivity of nominally 3.3 percent per meter ( 1 percent per foot), reporting an analog signal to the fire alarm panel.

\subsection{Instrumentation}

Thermocouples were installed to measure gas and surface temperatures within the room. Thermocouples, type $\mathrm{K}$ chromel-alumel, 28 gauge, were installed as shown in figure 5. One vertical array of ten thermocouples, TR1, was located between the patient beds, close to the head of patient bed number two, with thermocouples located at $0.61,0.76,0.91,1.2,1.4,1.5,1.7,2.0,2.3$, and 2.4 meters above the floor. A second vertical array of six thermocouples, TR2, was located approximately $1.1 \mathrm{~m}(3 \mathrm{ft} 7 \mathrm{in})$ from the doorway to the corridor with thermocouples located at $0.46,0.76,1.1,1.4,1.7$, and 2.0 meters above the floor. A thermocouple was placed at each patient head location, at the center of the bed, 0.15 $\mathrm{m}$ (6 in) above the surface. At each of the eight sprinkler locations shown in figure 3 , a thermocouple and thermocouple attached to a brass disk of known $\mathrm{RTI}=58(\mathrm{~m}-\mathrm{s})^{1 / 2}\left((105 \mathrm{ft}-\mathrm{s})^{1 / 2}\right)$, were placed to measure gas temperature at the sprinkler link and to obtain continuous thermal response data from a simulated sprinkler link of known RTI, respectively. A thermocouple was placed at each detector group. Another thermocouple was placed $0.05 \mathrm{~m}$ ( $2 \mathrm{in}$ ) below the ceiling in the center of the bathroom, and one thermocouple was placed $0.05 \mathrm{~m}$ ( 2 in) below the ceiling directly over the fire plume. The uncertainty in the thermocouple readings is $\pm 2.2^{\circ} \mathrm{C}$. [9]

Continuous measurements for carbon monoxide $(\mathrm{CO})$, carbon dioxide $\left(\mathrm{CO}_{2}\right)$, and oxygen $\left(\mathrm{O}_{2}\right)$ gas concentrations were made at elevations above the floor of $1.52 \mathrm{~m}(5 \mathrm{ft}$ ) (approximate head height for a person standing in the room), and at $0.91 \mathrm{~m}$ ( $3 \mathrm{ft}$ ) (approximate head height for a person lying in bed), between the patient beds as shown in figure 6 . At both locations, gas sampling occurred at a rate of approximately $0.04 \mathrm{l} / \mathrm{s}(5 \mathrm{cfh})$. The gas samples were drawn to analyzers through $6 \mathrm{~mm}(1 / 4$ in) copper tubing. Gases were passed through dry ice and desiccant traps to remove condensable vapors and particulate matter before entering $\mathrm{CO}, \mathrm{CO}_{2}$, and $\mathrm{O}_{2}$ analyzers in series. $\mathrm{CO}$ and $\mathrm{CO}_{2}$ concentrations were measured with non-dispersive IR meters manufactured by Infrared Industries, with a range of 0-2 percent $\mathrm{CO}$, and $0-20$ percent $\mathrm{CO}_{2} \cdot \mathrm{O}_{2}$ concentrations were measured with paramagnetic $\mathrm{O}_{2}$ meters manufactured by Sybron Taylor with a range of 0-25 percent. ${ }^{2}$

Water-cooled heat flux meters measured the total heat flux to each patient head location. The meter at the patient bed \#1, nearest the fire, was installed facing the fire, whereas the heat flux meter at patient bed \#2 was installed pointing towards the ceiling, since it was expected that this location would receive a higher flux from the hot layer than from the flames. A radiometer was located between the patient bed

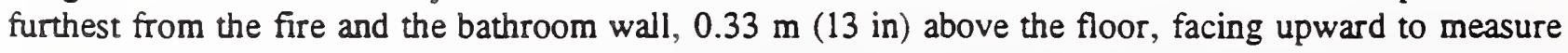
radiation only from the ceiling and the hot gas layer. Locations of heat flux meters are shown in figure 6.

2 Certain commercial equipment, instruments, or materials are identified in this paper in order to adequately specify the experimental procedure. Such identification does not imply recommendation or endorsement by the National Instiute of Standards and Technology, nor does it imply that the materials or equipment identified are necessarily the best available for the purpose. 


\subsection{FUEL}

A wood crib was chosen as the fuel package for the series of flaming fire tests. Wood cribs are used frequently in fire suppression research and standard testing. Cribs provide a challenge to the sprinkler in that they contain both shielded and exposed fuel surfaces.[10,11]

In selecting a fire, it was theorized that there exists a flaming fire size that challenges the tenability of the space, in that it burns for a while allowing the accumulation of smoke and toxic gases before the temperature is sufficient to activate the sprinklers. A large fire would activate the sprinklers rapidly when conditions were less severe in the room, and a small fire would not endanger the room occupants. It was determined through gas burner tests that a $60 \mathrm{~kW}$ steady state fire with the door closed posed the greatest challenge to the tenability of the space. An open door case was run to show a comparison, although such a fire probably would not reach untenable conditions in a ventilated room.

Oxygen consumption calorimetry tests in an instrumented hood [12] were used to determine what size wood crib would produce a nominal $60 \mathrm{~kW}$ rate of heat release. Before testing, each wood crib was oven dried until the moisture content was between 4 and 5 percent. Heptane, $(100 \mathrm{~m} \ell)$, with a burning time of approximately 300 seconds, was used to ignite the wood crib. The selected wood crib fuel array is illustrated in figure 7. Each crib used measured $0.61 \times 0.46 \times 0.15 \mathrm{~m}$ high $(24 \times 18 \times 6$ in high $)$, weighed between $7.5-8.2 \mathrm{~kg}$ (16.5-18 lbs), and was constructed of $38 \mathrm{~mm}(1-1 / 2$ in) sticks, spaced 38 $\mathrm{mm}$ (1-1/2 in) apart. The fuel was chosen to provide a prescribed amount of heat (not smoke) for the sprinkler study.

Cribs were burned on a load cell to obtain mass loss rate data for each test; this data was later used to estimate the rate of heat released from each fire over the course of the test. A value for the heat release rate was calculated by multiplying the mass loss rate by the net heat of combustion of wood, 12 $\mathrm{MJ} / \mathrm{kg}$. [13] This assumes that the heat of combustion does not change with the charring of the wood or the heat feedback from the upper layer, which are adequate assumptions for these fires. The heat release rate was similar for all tests, peaking around $90 \mathrm{~kW}$. Heat release rate curves are given in figure 8. The rate of heat release was similar for all fires. In the experiments, most sprinklers operated well before the fire reached its peak heat release rate. Heat release rates from the room fires are compared to a free burn of the wood crib fuel package. A heat release rate curve was not obtained for the shielded fire test.

\subsection{TEST SCENARIOS}

Test scenarios were selected in consultation with the sponsor and the ad hoc advisory committee. Test scenarios were selected to address the location and type of sprinklers in the hospital patient room, the effect of privacy curtains around the beds, the effect of a shielded fire, the performance of a sidewall sprinkler, and the effect of the room door position on small flaming fires in a hospital patient room. In all four tests, the bathroom door remained fully open. In the closed door tests, there was still a space of $2.0 \mathrm{~mm}$ under the door, providing for some leakage.

The four test scenarios are identified as follows:

1) Closed door test 
In the first test conducted, the room door remained closed throughout the test duration. The room was protected by an extended coverage sidewall sprinkler installed on the east wall furthest from the wood crib fire at location S1, as shown in figure 3. This test was designed to provide activation orders of the various sprinklers and detectors in terms of location and type, and conditions in the room at time of activation of each sprinkler.

\section{2) Closed door privacy curtain test}

In the second test, the room door also remained fully closed throughout the test duration. The privacy curtain midway between the beds was closed. Details of the privacy curtain installation are given above in the experimental facility section. The room was protected by an extended coverage sidewall sprinkler installed on the east wall furthest from the fire at location S1, as shown in figure 3 . This test was designed to measure the effect of a privacy curtain on the activation time of the various sprinklers, the ability of the extended coverage sidewall sprinkler to extinguish a fire on the other side of the curtain, and the effect of the privacy curtain on the conditions within the room at time of activation.

\section{3) Open door privacy curtain test}

This test set-up replicated that of the previous test, with the same curtain between the beds, except the room door was fully open instead of fully closed. The room was protected by an extended coverage sidewall sprinkler installed on the east wall furthest from the crib fire at location S1 as shown in figure 3. This test was designed to measure the effect of the room door opening position on the activation times of the sprinklers and detectors and on the conditions at activation.

\section{4) Closed door shielded fire test}

Test four was conducted with the room door closed. A non-combustible horizontal shield was placed $0.23 \mathrm{~m}$ ( $9 \mathrm{in}$ ) above the crib, to measure the effect of the shield on crib suppression and conditions within the room. The shield is pictured in figure 9 . The shield was $68 \mathrm{~cm}$ (27 in) above floor level, which is $18 \mathrm{~cm}(7 \mathrm{in})$ above the top of the crib. It measured $0.76 \times 0.91 \mathrm{~m}(2.5 \times 3 \mathrm{ft})$, and completely covered the top of the crib. The room was protected by an extended coverage sidewall sprinkler installed on the east wall furthest from the crib fire at location S1 as shown in figure 3. The purpose of the closed door shielded fire test was to determine the performance of the sidewall sprinkler in the case where there was no direct water impingement on the burning fuel. The room conditions were measured before and after water flow from the QR-EC sidewall sprinkler.

\subsection{TEST DESCRIPTIONS}

\subsection{Closed door test}

Table 1 lists the order of sprinkler and smoke detector actuation and the temperatures measured on thermocouple array TR1, between the patient beds at elevations of $1.5 \mathrm{~m}(5 \mathrm{ft})$ and $0.91 \mathrm{~m}(3 \mathrm{ft})$ above the floor. Figure 10 shows temperatures in the room measured on the thermocouple array between the patient beds and on the thermocouple array in the patient room entrance way.

The heptane pool fire was ignited between the two sticks furthest from the bed lengthwise and in the center of the crib width wise as shown in figure 7. The ignition location remained the same for all four tests. Test observations show that at the time of alarm of the first detector, an ionization type on the 
south wall between the beds, the flame height was $0.15 \mathrm{~m}(0.5 \mathrm{ft})$ above the surface of the crib, the heptane was burning but the crib had not yet become involved. The smoke layer was not yet visible.

At the time of activation of the first sprinkler, a QR sidewall over the head of the patient bed closest to

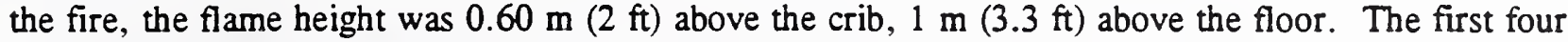
of nine sticks in the crib were fully involved, and the heptane had burned out. The visible smoke layer had not yet reached the position in the room being visually monitored, $1.5 \mathrm{~m}(5 \mathrm{ft})$ above the floor.

The visible smoke layer reached $1.5 \mathrm{~m}(5 \mathrm{ft})$ above floor level between 380 and 400 seconds after ignition, prior to activation of both the QR-EC sidewall sprinkler, on the east wall near the bathroom, and the first photoelectric smoke detector which activated at 439 seconds.

The QR-EC sidewall sprinkler activated 579 seconds after ignition. The visible smoke layer was $0.36 \mathrm{~m}$ (1.2 ft) above floor level at the time of activation of the QR-EC sidewall sprinkler. Two-thirds of the crib was fully involved at this time, the flame height was approximately $0.61 \mathrm{~m}(2 \mathrm{ft})$ above the surface of the crib.

Visual test records indicate that water flow from the QR-EC sidewall sprinkler reduced the fire to approximately half its size within the first 20 seconds, and provided full extinguishment of visible flaming of the crib in approximately 120 seconds. The temperature in the room reached a maximum of $133^{\circ} \mathrm{C}$ $\left(271^{\circ} \mathrm{F}\right)$, just prior to water flow, measured on thermocouple array TR1, between the beds, at an elevation of $2.3 \mathrm{~m}(7.5 \mathrm{ft})$ above the floor. The temperature at $1.5 \mathrm{~m}(5 \mathrm{ft})$ above the floor reached $76{ }^{\circ} \mathrm{C}\left(169^{\circ} \mathrm{F}\right)$ and the temperature at $0.91 \mathrm{~m}(3 \mathrm{ft})$ above the floor reached $40^{\circ} \mathrm{C}\left(104{ }^{\circ} \mathrm{F}\right)$ just prior to water flow.

\subsection{Closed door privacy curtain test}

Table 2 lists the order of sprinkler and smoke detector operation and the temperatures measured on the thermocouple array TR1, between the patient beds at elevations of $0.91 \mathrm{~m}(3 \mathrm{ft})$ and $1.5 \mathrm{~m}(5 \mathrm{ft})$ above the floor. Figure 11 shows temperanures in the room measured on the thermocouple array between the patient beds and the thermocouple array in the patient room entrance way. With the privacy curtain drawn, thermocouple array TR1, between the beds, was located on the opposite side of the privacy curtain from the fire.

The heptane pool fire was ignited as shown in figure 7. Test observations show that at the time of alarm of the first detector, an ionization type on the south wall between the beds, the flame height was $0.23 \mathrm{~m}$ $(0.75 \mathrm{ft})$ above the surface of the crib, $0.74 \mathrm{~m}(3.2 \mathrm{ft})$ above floor level. The fire was mostly heptane with a small amount of crib involvement.

At the time of activation of the first sprinkler, a QR sidewall over the head of the patient bed closest to the fire, three sticks of the crib were fully involved, the flame height was $0.61 \mathrm{~m}(2 \mathrm{ft})$ above the crib and the heptane had burned out.

At the time of activation of the photoelectric detectors, all of which alarmed within 16 seconds of each other, five sticks of the crib were fully involved. The visible smoke layer had reached the floor just prior, about 570 seconds after ignition. 
The QR-EC sidewall sprinkler (S1), activated 762 seconds after ignition, slightly more than 180 seconds after the smoke layer reached the floor. At this time, 6 of the nine crib sticks were fully involved, and the flame height was approximately $0.61 \mathrm{~m}(2 \mathrm{ft})$ above the crib surface.

Visual test records indicate that water flow from the QR-EC sidewall sprinkler reduced the fire to approximately half its size within the first 30 seconds, in 210 seconds, the fire was reduced to approximately 10 percent, and in a little over 240 seconds, the sprinkler was able to extinguish the fire. This was about twice the time to extinguishment of visible flame without the privacy curtain. The temperature in the room reached a maximum of $133^{\circ} \mathrm{C}\left(271{ }^{\circ} \mathrm{F}\right)$, measured on thermocouple array TR1, between the beds, $2.3 \mathrm{~m}(7.5 \mathrm{ft})$ above floor level just prior to water flow. The temperature at $1.5 \mathrm{~m}(5$ $\mathrm{ft}$ ) above the floor reached $77^{\circ} \mathrm{C}\left(171^{\circ} \mathrm{F}\right)$ and the temperature at $0.91 \mathrm{~m}(3 \mathrm{ft})$ above the floor reached $48^{\circ} \mathrm{C}\left(118^{\circ} \mathrm{F}\right)$ just prior to water flow.

\subsection{Open door privacy curtain test}

Table 3 lists the order of sprinkler and smoke detector operation and the temperatures measured on the thermocouple array TR1, between the patient beds at elevations of $0.91 \mathrm{~m}(3 \mathrm{ft})$ and $1.5 \mathrm{~m}(5 \mathrm{ft})$ above the floor. Figure 12 shows temperatures in the room measured on the thermocouple array between the patient beds and the thermocouple array in the patient room entrance way. With the privacy curtain drawn, thermocouple array TR1, between the beds, was located on the opposite side of the privacy curtain as the fire.

The heptane pool fire was ignited as shown in figure 7. Test observations show that at the time of alarm of the first detector, an ionization type on the south wall between the beds, the flame height was 0.38 $0.46 \mathrm{~m}(1.25-1.5 \mathrm{ft})$ above the surface of the crib, $0.88-0.97 \mathrm{~m}(2.9-3.2 \mathrm{ft})$ above floor level. The fire was mostly heptane with very little crib involvement.

At the time of activation of the first sprinkler, a QR sidewall over the head of the patient bed closest to the fire, three sticks of the crib were fully involved, the flame height was $0.68 \mathrm{~m}(2.25 \mathrm{ft})$ above the crib and the heptane had burned out. At the time of activation of the first photoelectric detector, on the ceiling in the center of the room, four sticks of the crib were fully involved.

The QR-EC sidewall sprinkler activated 820 seconds after ignition. At this time, six of the nine crib sticks were fully involved, and the flame height was approximately $0.61 \mathrm{~m}(2 \mathrm{ft})$ above the crib surface. Visual test records indicate that water flow from the QR-EC sidewall sprinkler reduced the fire to half the visible flame within 210 seconds after the sprinkler activated, the flame was reduced to 10 percent, and in a little over 240 seconds, the sprinkler was able to extinguish the fire. The temperature in the room reached a maximum of $131^{\circ} \mathrm{C}\left(268{ }^{\circ} \mathrm{F}\right)$, just prior to water flow, measured on the thermocouple array, TR1 between the beds, $2.3 \mathrm{~m}(7.5 \mathrm{ft})$ above floor level. The temperature at $1.5 \mathrm{~m}(5 \mathrm{ft})$ above the floor reached $59{ }^{\circ} \mathrm{C}\left(138{ }^{\circ} \mathrm{F}\right)$ and the temperature at $0.91 \mathrm{~m}(3 \mathrm{ft})$ above the floor reached $31{ }^{\circ} \mathrm{C}$ $\left(88{ }^{\circ} \mathrm{C}\right)$ just prior to water flow.

\subsection{Closed door shielded fire test}

Table 4 lists the order of sprinkler and smoke detector operation and the temperatures measured on the thermocouple array TR1, between the patient beds at elevations of $0.91 \mathrm{~m}(3 \mathrm{ft})$ and $1.5 \mathrm{~m}(5 \mathrm{ft})$ above the floor. Figure 13 shows the temperatures in the room measured on the thermocouple array between the patient beds and the thermocouple array in the patient room entrance way. 
The heptane pool fire was ignited as shown in figure 7. The flame reached the top of the shield in under 10 seconds, and started to extend out and around the shield in approximately 90 seconds. At the time of activation of the first smoke detector, an ionization type on the south wall between the beds, the fire was mostly heptane. At this time the visible smoke layer had not yet reached the position in the room being visually monitored, $1.5 \mathrm{~m}(5 \mathrm{ft})$ above the floor.

At the time of activation of the first sprinkler, a QR sidewall over the head of the patient bed closest to the fire, the heptane had burned out, the first four of the nine sticks were fully involved. The visible smoke layer was just above the position in the room being visually monitored, $1.5 \mathrm{~m}(5 \mathrm{ft})$ above the floor.

The smoke layer reached the $1.5 \mathrm{~m}(5 \mathrm{ft})$ position at 390 seconds, and by 450 seconds, the visible smoke reached the top of the foot boards on the beds, $0.76 \mathrm{~m}(2.5 \mathrm{ft})$ above the floor. At approximately 480 seconds from ignition, the smoke layer had reached the bottom of the foot boards, $0.36 \mathrm{~m}(1.2 \mathrm{ft})$ off the floor, and by 570 seconds the smoke layer reached the floor.

The QR-EC sidewall sprinkler (S1), activated 700 seconds after ignition. At this time, the smoke layer had dropped to the floor level. The flame height at this time was $0.61 \mathrm{~m}(2 \mathrm{ft})$ above the surface of the crib which was fully involved. The heptane had burned out 300 seconds after ignition.

Visual test records indicate that water flow from the QR-EC sidewall sprinkler reduced the fire to approximately half its size, the QR-EC sidewall sprinkler, however, was not able to extinguish the fire. Sprinkler flow was allowed to continue for 1260 seconds after activation and then the fire was manually extinguished. The temperature in the room reached a maximum of $135^{\circ} \mathrm{C}\left(275^{\circ} \mathrm{F}\right)$ just prior to water flow, measured on thermocouple array TR1 between the patient beds, $2.3 \mathrm{~m}(7.5 \mathrm{ft})$ above the floor. The temperature at $1.5 \mathrm{~m}(5 \mathrm{ft})$ above the floor reached $111^{\circ} \mathrm{C}\left(232^{\circ} \mathrm{F}\right)$ and the temperature at $0.91 \mathrm{~m}$ $(3 \mathrm{ft})$ above the floor reached $78^{\circ} \mathrm{C}\left(172^{\circ} \mathrm{F}\right)$ just prior to water flow.

\subsection{RESULTS AND DISCUSSION}

Temperature, heat flux, carbon dioxide, carbon monoxide, and oxygen concentrations were continuously measured in order to assess tenability of the hospital patient room. Temperature at head height for a person standing in the room $(1.5 \mathrm{~m})$, and for a person lying in bed $(0.91 \mathrm{~m})$ are discussed relative to the activation times of each device. A discussion of the gas concentrations and heat flux follows. Gas concentrations are presented in terms of maximum values reached prior to sprinkler activation and after water flow from the extended coverage sidewall sprinkler. Heat flux is examined in terms of maximum values achieved at time of activation. Overall tenability of the room, in terms of each sprinkler is then evaluated with TENAB, a subroutine of HAZARD I.[14] Time/temperature data at the sprinkler location from preignition to completion of the test is reported in the Appendix. Numbering of thermocouples and disks at sprinkler locations follows that of figure 5.

\subsection{General observations}

1) In all cases except the completely shielded fire, the QR-EC sidewall sprinkler was able to extinguish the fire. In the closed door test, the sprinkler was able to completely extinguish the fire in approximately 120 seconds, in each of the privacy curtain tests, extinguishment of visible flame was achieved in a little over 240 seconds. In the shielded fire scenario, the sprinkler was not able to extinguish the fire. The fire was manually extinguished 1260 seconds after the initiation of water flow from the sprinkler. 
2) The ionization detectors in all locations responded well in advance of any of the automatic sprinklers. The initial detector activation was received between 232-377 seconds prior to activation of the first sprinkler, and 552-722 seconds prior to activation of the QR-EC sidewall sprinkler.

3) Concealed quick response sprinklers $(S 9, S 10)$, UL listed as quick response sprinklers, took between 55 and 158 seconds longer to operate than their quick response counterparts (S2, S4). Concealed sprinklers took between 60 to 90 seconds to operate after the release of the cover plate from the sprinkler assembly.

4) Concealed quick response sprinkler, S10, operated between 8 and 99 seconds in advance of the standard response sprinkler $0.1 \mathrm{~m}$ away (S8), except in the case of the shielded fire test, where sprinkler S8 responded 16 seconds in advance of sprinkler S10.

5) The standard response pendent sprinkler (S8), operated in advance of the QR-EC sidewall sprinkler (S1) in all fire tests.

6) In these tests, photoelectric detectors responded much slower than the ionization detectors, and in some cases, one or more quick response sprinklers activated prior to response of any of the photoelectric detectors.

7) Oxygen concentrations did not drop below $19 \%$, carbon dioxide concentrations did not go above $2 \%$, and carbon monoxide concentrations did not exceed $500 \mathrm{ppm}$.

8) Temperature at time of activation of the quick response sprinklers was at or below $77{ }^{\circ} \mathrm{C}\left(171^{\circ} \mathrm{F}\right)$ at the five foot level and below $48^{\circ} \mathrm{C}\left(118^{\circ} \mathrm{F}\right)$ at the three foot level between the patient beds, in all tests with the exception of the shielded fire scenario where temperatures at the five and three foot levels reached $111^{\circ} \mathrm{C}\left(232{ }^{\circ} \mathrm{F}\right)$, and $78^{\circ} \mathrm{C}\left(172{ }^{\circ} \mathrm{F}\right)$ respectively.

\subsection{Time and Temperature at Time of Activation}

\subsubsection{Quick response sprinklers}

Test results show that the first sprinkler to operate was always S12, the quick response (QR) sidewall sprinkler on the south wall, over the head of the patient bed closest to the fire (figure 3). The QR-EC sidewall sprinkler, S1, on the east wall at the far end of the room furthest from the flaming wood crib fire, was in all four tests the slowest of the quick response sprinklers to operate. In all four tests, the QR-EC sidewall sprinkler at location S1 operated after the standard pendent at S8 above the foot of patient bed number one. S6, the standard sidewall, across the foot of patient bed $\# 2$, did not activate prior to water flow from the QR-EC sidewall, except in the shielded fire test.

For most of the quick response sprinklers tested the temperature at the $1.5 \mathrm{~m}(5 \mathrm{ft})$ elevation at the time of activation of the sprinkler did not exceed $77^{\circ} \mathrm{C}\left(171{ }^{\circ} \mathrm{F}\right)$, and did not exceed $48{ }^{\circ} \mathrm{C}\left(118^{\circ} \mathrm{F}\right)$ at the

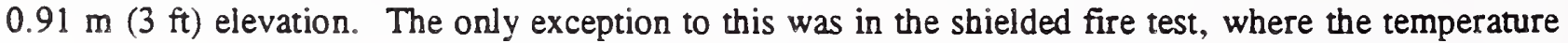
at the time of activation of the QR-EC sidewall sprinkler was $111^{\circ} \mathrm{C}\left(232^{\circ} \mathrm{F}\right)$ at the $1.5 \mathrm{~m}(5 \mathrm{ft})$ elevation, and $78^{\circ} \mathrm{C}\left(172{ }^{\circ} \mathrm{F}\right)$ at the $0.91 \mathrm{~m}(3 \mathrm{ft})$ elevation. 


\subsubsection{Concealed quick response sprinklers}

Table 5 shows the comparison of response times between the standard response pendant at location S8, and the concealed quick response sprinkler at location S10. It also shows the difference in response times between the concealed quick response sprinklers at locations $S 10$ and S9, and their quick response exposed sprinklers at locations S4 and S2. In general, the concealed quick response sprinklers took between 55 seconds and 158 seconds longer to operate in response to this fire than their quick response counterparts. In the open door privacy curtain test, however, the concealed quick response sprinkler at location S10 operated 23 seconds faster than its quick response counterpart at location S4. This can be attributed to the fact that the concealed quick response sprinklers have operating temperatures of $57^{\circ} \mathrm{C}$ $\left(135^{\circ} \mathrm{F}\right)$ for each the bulb and cover plate solder, whereas both the quick response pendent and the standard response pendent have operating temperatures of $68^{\circ} \mathrm{C}\left(155^{\circ} \mathrm{F}\right)$. In the open door scenario where there is a slower build-up of temperature in the room than in the closed door test, the sprinkler link has time to heat to $57{ }^{\circ} \mathrm{C}$ after the cover plate drops off before the $\mathrm{QR}$ link heats to $68{ }^{\circ} \mathrm{C}$. The concealed quick response pendent sprinkler at S10 operated between 8 and 99 seconds in advance of its standard response counterpart at S8. An exception to this can be seen in the shielded fire test, where the standard response sprinkler activated 16 seconds in advance of the concealed quick response pendent 0.10 m (4 in) away. The concealed quick response sprinklers in the closed door tests took approximately 60 seconds to operate after their cover plates dropped off, this time increased to approximately 90 seconds in the open door test.

The largest difference in temperature at the time of activation of the concealed quick response sprinklers compared to their quick response counterparts occurred in the closed door test. The $1.5 \mathrm{~m}(5 \mathrm{ft})$ temperature at the time of activation of the concealed quick response sprinkler at location S 10 was $64{ }^{\circ} \mathrm{C}$ $\left(146^{\circ} \mathrm{F}\right)$ compared to $49^{\circ} \mathrm{C}\left(121^{\circ} \mathrm{F}\right)$ at the time of activation of the quick response sprinkler at location S4. The corresponding $0.91 \mathrm{~m}(3 \mathrm{ft})$ temperatures were $35^{\circ} \mathrm{C}\left(95^{\circ} \mathrm{F}\right)$ and $29^{\circ} \mathrm{C}\left(84^{\circ} \mathrm{F}\right)$ respectively. At the time of activation of the concealed quick response sprinkler at location $\mathrm{S} 9$, the $1.5 \mathrm{~m}(5 \mathrm{ft})$ temperature was $75^{\circ} \mathrm{C}\left(168^{\circ} \mathrm{F}\right)$ compared to $53^{\circ} \mathrm{C}\left(128^{\circ} \mathrm{F}\right)$ at the time of activation of S2 its quick response counterpart. The corresponding $0.91 \mathrm{~m}(3 \mathrm{ft})$ temperatures were $40^{\circ} \mathrm{C}\left(103{ }^{\circ} \mathrm{F}\right)$ and $31{ }^{\circ} \mathrm{C}$ $\left(88^{\circ} \mathrm{F}\right)$ respectively as can be seen in table 1.

\subsubsection{Standard response sprinklers}

The standard response pendent sprinkler at S8 operated 76 to 142 seconds slower than its quick response counterpart at S4, and 8 to 99 seconds slower than it's concealed quick response counterpart at $S 10$ as shown in table 5. It is interesting to note that in all four tests, the standard pendent at $\mathbf{8}$ responded prior to the QR-EC sidewall sprinkler at S1. In all but the shielded fire test, the standard response sidewall sprinkler (S6) on the north wall across from the foot of the patient bed furthest from the fire, did not activate during the test due to cooling by water flow from the prior activating QR-EC sidewall sprinkler on the east wall at $S 1$.

The standard pendent at location S8 operated after its quick response and concealed quick response counterparts, sprinklers S4 and S10 respectively in all except the shielded fire test. The greatest difference in temperature occurred in the closed door test. The temperature at the $1.5 \mathrm{~m}(5 \mathrm{ft})$ level at the time of activation of the standard response sprinkler at location $S 8$ was $70^{\circ} \mathrm{C}\left(158{ }^{\circ} \mathrm{F}\right)$ compared to 
$49^{\circ} \mathrm{C}\left(120^{\circ} \mathrm{F}\right)$ at the time of activation of $\mathrm{S} 4$, it's quick response counterpart. The corresponding 0.91 m (3 ft) temperatures were $39^{\circ} \mathrm{C}\left(102^{\circ} \mathrm{F}\right)$ and $29^{\circ} \mathrm{C}\left(84^{\circ} \mathrm{F}\right)$ respectively as can be seen in table 1.

\subsubsection{Ionization Detectors}

Data for smoke detector/sensor response presented in tables 1-4 showed that in all of the fire tests, the ionization detectors in all locations responded in advance of any of the automatic sprinklers. The initial detector activation was received between 232-377 seconds prior to activation of the first sprinkler, and 552-722 seconds prior to activation of the QR-EC sidewall sprinkler. The first detector to respond was the ionization detector at Group A, located on the south wall between the beds. This was followed by the ionization detector at Group D, located on the ceiling in the center of the room. This sequence remained the same for all tests. There was a 77 to 130 second time span from the time of activation of the first ionization detector to the time of activation of the fourth ionization detector.

Temperature at the time of activation of the ionization smoke detectors was at or below $34{ }^{\circ} \mathrm{C}\left(93{ }^{\circ} \mathrm{F}\right)$ at the $1.5 \mathrm{~m}(5 \mathrm{ft})$ elevation and at or below $29{ }^{\circ} \mathrm{C}\left(84{ }^{\circ} \mathrm{F}\right)$ at the $0.91 \mathrm{~m}(3 \mathrm{ft})$ elevation for all four detector locations tested in all four fire tests. The temperature of the gas stream in the detector area at the time of activation of the ionization detectors was only $3{ }^{\circ} \mathrm{C}-7^{\circ} \mathrm{C}$ above ambient temperature, which was $22{ }^{\circ} \mathrm{C}$.

\subsubsection{Photoelectric Detectors/Sensors}

The photoelectric detectors/sensors were slower to respond than the ionization detectors, and did not in all cases actuate in advance of the quick response sprinklers. This is in part due to the ignition source/fuel type. Heptane was used to ignite the wood crib which was chosen to provide a prescribed amount of heat not smoke. The first photoelectric detector to respond was, in two out of the four tests, $D 7$, on the ceiling in the center of the room. In each of the four tests, there was between 16 to 56 seconds between the time to alarm of the first photoelectric detector and the time to alarm of the fourth photoelectric detector.

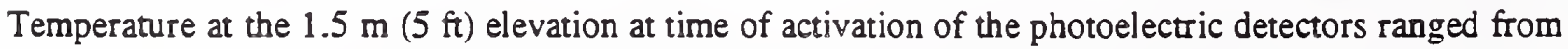
$32{ }^{\circ} \mathrm{C}\left(89^{\circ} \mathrm{F}\right)$, at the time of activation of $\mathrm{D} 7$, on the ceiling in the center of the room, during the open door, sprinkler protection privacy curtain test, to $56^{\circ} \mathrm{C}\left(133^{\circ} \mathrm{F}\right)$, at the time of activation of D5, on the ceiling, near room door during the closed door test. Temperatures at the $0.91 \mathrm{~m}(3 \mathrm{ft})$ elevation at the time of activation of the photoelectric detectors ranged from $27^{\circ} \mathrm{C}\left(81 \mathrm{~F}^{\circ}\right)$, at the time of activation of all four photoelectric detectors in the open door privacy curtain test, to $40^{\circ} \mathrm{C}\left(104{ }^{\circ} \mathrm{F}\right)$ at the time of activation of D5, on the ceiling, near the room door, during the shielded fire test.

\subsection{Gas Concentrations}

Continuous measurements of oxygen, carbon monoxide, and carbon dioxide were taken at the $1.5 \mathrm{~m}$ (5 $\mathrm{ft})$ and $0.91 \mathrm{~m}(3 \mathrm{ft})$ elevations between the patient beds as shown in figure 6 . Concentrations of oxygen and carbon dioxide followed the expected trends, with the carbon dioxide concentration increasing in relationship to the decrease in oxygen concentration. Oxygen concentration remained greater than 19 percent in all of the fire tests, and the carbon dioxide concentrations reached a maximum of two percent. Carbon dioxide and carbon monoxide concentrations are reported in table 6, before and after sprinkler activation. Carbon monoxide levels were at the baseline, within the measurable uncertainty of the gas 
meters which is less than $100 \mathrm{ppm}$, for the closed door test, and the closed door privacy curtain test. In the open door privacy curtain test, $\mathrm{CO}$ reached a maximum of $100 \mathrm{ppm}$ before water flow and a maximum of $400 \mathrm{ppm}$ just after water flow from the QR-EC sidewall sprinkler. In the closed door shielded fire test, $C O$ levels reached a maximum of $400 \mathrm{ppm}$ before water flow and a maximum of $500 \mathrm{ppm}$ after water flow from the QR-EC sidewall sprinkler.

\subsection{Heat Flux}

Measured heat flux at each patient location is presented in table 6. The fluxes reported for the patient beds are total flux, radiation and convection. The flux meter over patient bed \#1 was installed facing the fire, whereas the flux meter placed over patient bed $\# 2$, was installed facing the ceiling. Flux is specific to the location relative to the fire. The total flux measured at patient bed $\# 2$, facing the ceiling, is lower for the open door test than the closed door tests, as there is little development of a hot layer in the open door case. Heat flux is somewhat a function of the fuel. The radiation measurement from the ceiling hot gas layer was $0.1 \mathrm{~kW} / \mathrm{m}^{2}$ for the closed door tests. No ceiling radiation was measured for the open door test.

\subsection{Tenability, HAZARD I, and the N-Gas model}

The tenability of the room can be determined from the above presented data in terms of which sprinklers actuated before the patient's life was threatened. To determine this, one must know the effect of temperature, heat flux, and gas concentration on the patient.

In 1989, the Center for Fire Research at the National Institute of Standards and Technology released HAZARD I, a method for quantifying the hazards to occupants of buildings from fires. HAZARD I uses a sequence of computer software procedures to calculate the development of hazardous conditions over time, to calculate the time needed by building occupants to escape under those conditions, and to estimate the resulting loss of life based on assumed occupant behavior and tenability criteria. The module of HAZARD I which calculates the impact of exposure to the occupants is called TENAB.

Years of research and review of current literature went into determining the tenability criteria for TENAB. This report does not review that literature. However, a summary of previous research into tenability criteria, an extensive list of references, and the basis for the threshold values used and the derivation of the equations on which the toxicity calculation is based, are provided in the technical reference volume in the chapter on tenability limits.[14] In TENAB, lethality is determined from temperature and toxicity, along with potential incapacitation from burns due to radiant heat exposure.

In HAZARD I, death from temperature exposure is taken to occur at $100{ }^{\circ} \mathrm{C}$. Heat flux is not a lethality condition. The fractional exposure dose (FED), a toxicity parameter, is introduced. The FED represents the fraction of the lethal concentration that an individual has been exposed to over time. The FED parameter combines the effects and interactions of the gases $\mathrm{CO}, \mathrm{CO}_{2}$, and $\mathrm{HCN}$, along with the effect of diminished oxygen. The model on which the FED calculation is based is referred to as the N-Gas model. The FED parameter is calculated as a function of the average concentrations of carbon monoxide, carbon dioxide, and oxygen over the time interval of exposure. Incapacitation of a human being is taken to occur when the FED parameter equals 0.5 , and death is taken to occur when the FED parameter equals 1.0. The uncertainty in the FED values determined from animal experiments range from no deaths observed at FED values below 0.9, to death of all animals exposed at FED values greater than 1.3. 
FED values calculated with TENAB are zero for all tests in this series. This means that TENAB does not predict any deaths due to toxicity for these room fires. This is due to the fact that the measured concentration of carbon monoxide is less than $1700 \mathrm{ppm}$, which is the threshold value. The carbon dioxide term is a "correction" to the carbon monoxide term, and becomes zero when the carbon monoxide term is zero. In TENAB, the oxygen concentration must drop below 9.2 percent to affect the breathing of the patient.

The temperature criterion for death, $100{ }^{\circ} \mathrm{C}$, is exceeded only in the shielded fire test. In this test, the temperature criterion is exceeded at the $1.52 \mathrm{~m}(5 \mathrm{ft})$ level, by the sprinkler at location $S 6$, the standard sidewall across the foot of bed \#2, and the sprinkler at location $\mathrm{S1}$, the EC sidewall on the east wall near the bathroom. At these sprinkler locations, the temperature reaches $108^{\circ} \mathrm{C}$, and $111^{\circ} \mathrm{C}$, respectively. Temperatures at the $0.91 \mathrm{~m}(3 \mathrm{ft})$ level at these sprinkler locations remained below $100{ }^{\circ} \mathrm{C}$, at $75^{\circ} \mathrm{C}$ and $78^{\circ} \mathrm{C}$, respectively.

\subsection{CONCLUSION}

Sprinklers in all locations tested actuated before the patient's life would be threatened by this nominally $60 \mathrm{~kW}$ fire for the closed door, closed door privacy curtain, and open door privacy curtain tests. However, in the shielded fire test, the sprinklers at locations S6 and S1, the standard sidewall across from the foot of patient bed $\# 2$, and the EC sidewall, on the east wall near the bathroom, respectively, activated after the life safety criterion in HAZARD I with regard to temperature was exceeded. Ionization and photoelectric detectors in all locations alarmed before the patient's life would be threatened for all fire test scenarios conducted.

\subsection{ACKNOWLEDGEMENTS}

The author wishes to acknowledge the National Institutes of Health (NIH) for support of this research. Appreciation is extended to Simplex Time Recorder Co., who on behalf of the detector industry, donated the equipment and professional staff to allow the scope of this project to be expanded to include smoke detection. Appreciation also is extended to The Reliable Automatic Sprinkler Co. and The Viking Corporation for donations of sprinkler products, and to Tamatex Inc. for donation of the privacy curtains.

The author gratefully acknowledges J. Morehart, (NIH), D. Evans, W. Walton, Building and Fire Research Laboratory (BFRL), and H.E. Nelson, Hughes Associates, for their guidance and encouragement, as well as W. Rinkinen (BFRL), who assumed primary responsibility for construction and instrumentation of the test facility, and L. Desmarais and R. Roux, SET (Simplex) for their guidance and assistance in instrumentation, data acquisition and data analysis of the smoke detector portion of this study.

Thanks also are extended to J. Milke of the University of Maryland; K. Faulstich of the Veterans Administration; R. Fleming and K. Isman of the National Fire Sprinkler Association; and J. Drouin and L. Stanley of Simplex Time Recorder Co. for serving on an ad-hoc advisory committee. 


\subsection{REFERENCES}

1. O'Neil, J.G., and Hayes, W.D., "Full-Scale Fire Tests with Automatic Sprinklers in a Patient Room," National Bureau of Standards (U.S.) NBSIR 79-1749; June 1979.

2. O'Neil, J.G., and Hayes, W.D., "Full-Scale Fire Tests with Automatic Sprinklers in a Patient Room. Phase II," National Bureau of Standards (U.S.) NBSIR 80-2097; July 1980.

3. Walton, W. D., and Budnick, E.K., "Quick Response Sprinklers in Office Configurations: Fire Test Results," National Bureau of Standards (U.S.) NBSIR 88-3695; January 1988.

4. Bill, R. G., Jr., "An Evaluation of Extended Coverage, Sidewall Sprinklers and Smoke Detectors in a Hotel Occupancy," FMRC OM3N5.RA, Factory Mutual Research; Norwood, MA, May 1988.

5. NFPA 101, "Life Safety Code," in National Fire Codes, National Fire Protection Association, Quincy MA, 1991.

6. Bill, R. G., Jr., "The Response of Smoke Detectors to Smoldering-Started Fires in a Hotel Occupancy," FMRC OQOR4.RA, Factory Mutual Research; Norwood, MA, 1988.

7. NFPA 13, "Standard for the Installation of Sprinkler Systems," National Fire Codes, National Fire Protection Association, Quincy MA, 1989.

8. NFPA 72E, "Standard on Automatic Fire Detectors," National Fire Codes, National Fire Protection Association, Quincy MA, 1990.

9. The Temperature Handbook, Omega Engineering Inc., Stamford, CT, 1989.

10. Walton, W. D., "Suppression of Wood Crib Fires With Sprinkler Sprays: Test Results," National Bureau of Standards (U.S.) NBSIR 88-3696; January 1988.

11. UL 199, "Standard for Automatic Sprinklers for Fire Protection Service," Underwriters Laboratories Inc., Northbrook IL, Revision February 1990.

12. Parker, W.J., "Calculations of the Heat Release Rate by Oxygen Consumption for Various Applications." National Bureau of Standards (U.S.) NBSIR 81-2427-1; March, 1982.

13. Babrauskas, V., "Burning Rates," in DiNenno, P.J., ed.-in-chief, The Handbook of Fire Protection Engineering, National Fire Protection Association, Quincy MA, and Society of Fire Protection Engineers, Boston MA, $1^{\text {st }}$ edition, 1988.

14. Peacock, R. D., Jones, W.W., Bukowski, R.W., and Forney, L.C., "Technical Reference Guide for the HAZARD I Fire Hazard Assessment Method" National Institute of Standards and Technology (U.S.) NIST Handbook 146, Volume II, June 1991. 
Table 1 - Activation times, Closed door test

\begin{tabular}{|c|c|c|c|c|c|}
\hline $\begin{array}{l}\text { Device } \\
\text { Number }\end{array}$ & Description & $\begin{array}{c}\text { Activation } \\
\text { Time } \\
\text { min:s }\end{array}$ & $\begin{array}{c}\text { Activation } \\
\text { Time } \\
\text { s }\end{array}$ & $\begin{array}{l}1.5 \mathrm{~m}(5 \mathrm{ft}) \\
\text { Elevation } \\
\text { Between Beds } \\
\text { Temp }{ }^{\circ} \mathrm{C}\end{array}$ & $\begin{array}{l}0.91 \mathrm{~m}(3 \mathrm{ft}) \\
\text { Elevation } \\
\text { Between Beds } \\
\text { Temp }{ }^{\circ} \mathrm{C}\end{array}$ \\
\hline D2 & ion, south wall, between beds & $0: 27$ & 27 & 22 & 22 \\
\hline DE & ion, ceiling, center of room & $0: 36$ & 36 & 22 & 22 \\
\hline D4 & ion, east wall, bathroom & $2: 13$ & 133 & 24 & 22 \\
\hline D6 & ion, ceiling, near room door & $2: 37$ & 157 & 25 & 23 \\
\hline$S 12$ & QR sidewall, overhead bed \#1 & $5: 58$ & 358 & 38 & 26 \\
\hline S4 & QR pendent, above foot bed $\# 1$ & $7: 02$ & 422 & 49 & 29 \\
\hline S11 & QR sidewall, overhead bed $\sharp 2$ & $7: 11$ & 439 & 50 & 29 \\
\hline D7 & photo, ceiling, center of room & $7: 40$ & 439 & 52 & 30 \\
\hline DSC & $\begin{array}{l}\text { photo sensor, ceiling, near room } \\
\text { door }\end{array}$ & $7: 20$ & 440 & 52 & 30 \\
\hline S3 & QR-EC pendent, center of room & $7: 20$ & $44 ?$ & 52 & 30 \\
\hline D3 & photo, east wall, bathroom & $7: 21$ & 441 & 53 & 31 \\
\hline$S 2$ & QR pendent, above foot bed $\# 2$ & $7: 29$ & 449 & 53 & 31 \\
\hline DSE & photo sensor, ceiling, center of room & $7: 33$ & 453 & 54 & 31 \\
\hline DSB & photo sensor, east wall, bathroom & $7: 37$ & 457 & 55 & 31 \\
\hline D5 & photo, ceiling, near room door & $7: 40$ & 460 & 56 & 31 \\
\hline S5 & QR sidewall, across foot bed $\sharp 1$ & $8: 27$ & 507 & 63 & 35 \\
\hline S10 & $\begin{array}{l}\text { QR concealed pendent, above foot } \\
\text { bed } \# 1\end{array}$ & $8: 28$ & 508 & 64 & 35 \\
\hline 57 & QR sidewall, across foot bed $\# 2$ & $8: 59$ & 539 & 49 & 37 \\
\hline S8 & standard pendent, above foot bed $\# 1$ & $9: 09$ & 539 & 70 & 39 \\
\hline S9 & $\begin{array}{l}\text { QR concealed pendent, above foot } \\
\text { bed } \# 2\end{array}$ & $9: 22$ & 562 & 75 & 40 \\
\hline $\mathrm{SI}$ & QR-EC sidewall, east wall, bathroom & $9: 39$ & 579 & 76 & 40 \\
\hline S6 & standard sidewall, across foot bed $\# 2$ & - & - & - & - \\
\hline D1 $\mathrm{n} / \mathrm{a}$ & photo, south wall, between beds & - & - & - & - \\
\hline DSA & $\begin{array}{l}\text { photo sensor, south wall, between } \\
\text { beds }\end{array}$ & - & - & - & - \\
\hline
\end{tabular}


Table 2 - Activation times, Closed door privacy curtain test

\begin{tabular}{|c|c|c|c|c|c|}
\hline $\begin{array}{l}\text { Device } \\
\text { Number }\end{array}$ & Description & $\begin{array}{l}\text { Activation } \\
\text { Time } \\
\text { min: s }\end{array}$ & $\begin{array}{c}\text { Activation } \\
\text { Time } \\
\text { s }\end{array}$ & $\begin{array}{c}1.5 \mathrm{~m}(5 \mathrm{ft}) \\
\text { Elevation } \\
\text { Between Beds } \\
\text { Temp }{ }^{\circ} \mathrm{C}\end{array}$ & $\begin{array}{c}0.91 \mathrm{~m}(3 \mathrm{ft}) \\
\text { Elevation } \\
\text { Between Beds } \\
\text { Temp }{ }^{\circ} \mathrm{C}\end{array}$ \\
\hline D2 & ion, south wall, between beds & $0: 40$ & 40 & 25 & 25 \\
\hline D8 & ion, ceiling, center of room & $2: 03$ & 123 & 27 & 25 \\
\hline D4 & ion, east wall, bathroom & 2:05 & 125 & 27 & 25 \\
\hline D6 & ion, ceiling, near room door & $2: 44$ & 164 & 28 & 25 \\
\hline S12 & QR sidewall, overhead bed \#1 & $6: 57$ & 619 & 42 & 30 \\
\hline D5 & photo, ceiling, near room door & 9:29 & 569 & 54 & 31 \\
\hline$S 11$ & QR sidewall, overhead bed \#2 & $9: 35$ & 570 & 54 & 32 \\
\hline D7 & photo, ceiling, center of room & $9: 35$ & 575 & 54 & 35 \\
\hline D4 & photo, east wall, bathroom & $9: 41$ & 581 & 54 & 36 \\
\hline D1 & photo, south wall, between beds & $9: 45$ & 585 & 68 & 36 \\
\hline S4 & QR pendent, above foot bed $\# 1$ & $10: 19$ & 619 & 58 & 37 \\
\hline DSD & photo sensor, ceiling, center of room & $10: 33$ & 633 & 58 & 38 \\
\hline DSC & photo sensor, ceiling, near room door & $10: 48$ & 648 & 61 & 39 \\
\hline S5 & QR sidewall, across foot bed $\# 1$ & $10: 50$ & 695 & 61 & 39 \\
\hline DSA & $\begin{array}{l}\text { photo sensor, south wall, between } \\
\text { beds }\end{array}$ & $10: 52$ & 652 & 61 & 39 \\
\hline S3 & QR-EC pendent, center of room & $10: 59$ & 659 & 62 & 40 \\
\hline DSB & photo sensor, east wall, bathroom & $11: 05$ & 665 & 63 & 40 \\
\hline S2 & QR pendent, above foot bed $\# 2$ & $11: 12$ & 672 & 63 & 40 \\
\hline S10 & $\begin{array}{l}\text { QR concealed pendent, above foot } \\
\text { bed } \# 1\end{array}$ & $11: 27$ & 687 & 67 & 41 \\
\hline S7 & QR sidewall across foot bed $\# 2$ & $11: 34$ & 694 & 67 & 41 \\
\hline S\& & standard pendent, above foot bed $\# 1$ & $11: 35$ & 695 & 68 & 41 \\
\hline S9 & $\begin{array}{l}\text { QR concealed pendent, above foot } \\
\text { bed } \# 2\end{array}$ & $12: 33$ & 753 & 77 & 48 \\
\hline SI & QR-EC sidewall, east wall, bathroom & $12: 42$ & 762 & 77 & 48 \\
\hline S6 n/a & standard sidewall, across foot bed $\sharp 2$ & - & - & - & - \\
\hline
\end{tabular}


Table 3 - Activation times, Open door privacy curtain test

\begin{tabular}{|c|c|c|c|c|c|}
\hline $\begin{array}{l}\text { Device } \\
\text { Number }\end{array}$ & Description & $\begin{array}{l}\text { Activation } \\
\text { Time } \\
\text { min: s } \\
\end{array}$ & $\begin{array}{c}\text { Activation } \\
\text { Time } \\
\text { s }\end{array}$ & $\begin{array}{l}1.5 \mathrm{~m}(5 \mathrm{ft}) \\
\text { Elevation } \\
\text { Between } \\
\text { Beds } \\
\text { Temp }{ }^{\circ} \mathrm{C}\end{array}$ & $\begin{array}{c}0.91 \mathrm{~m}(3 \mathrm{ft}) \\
\text { Elevation } \\
\text { Between Beds } \\
\text { Temp }{ }^{\circ} \mathrm{C}\end{array}$ \\
\hline D2 & ion, south wall, between beds & $1: 57$ & 117 & 27 & 26 \\
\hline D8 & ion, ceiling, center of room & $2: 19$ & 139 & 27 & 26 \\
\hline D3 & ion, east wall, bathroom & $2: 24$ & 144 & 28 & 26 \\
\hline D6 & ion, ceiling, near room door & $3: 14$ & 194 & 28 & 26 \\
\hline$\$ 12$ & QR sidewall, overhead bed \#1 & $5: 49$ & 349 & 31 & 27 \\
\hline D7 & photo, ceiling, center of room & $6: 53$ & 413 & 32 & 27 \\
\hline D1 & photo, south wall, between beds & 7:01 & 421 & 32 & 27 \\
\hline DSA & photo sensor, south wall, between beds & $7: 18$ & 438 & 32 & 29 \\
\hline DSD & photo sensor, ceiling, center of room & $7: 22$ & 442 & 32 & 27 \\
\hline D8 & photo, ceiling, near room door & $7: 25$ & 445 & 32 & 27 \\
\hline DSB & photo sensor, east wall, bathroom & $7: 27$ & 447 & 33 & 27 \\
\hline D3 & photo, east wall, bathroom & $7: 49$ & 469 & 33 & 27 \\
\hline DSC & photo sensor, ceiling, near room door & $8: 02$ & 482 & 34 & 27 \\
\hline $\mathrm{S} 10$ & $\begin{array}{l}\text { QR concealed pendent, above foot bed } \\
\$ 1\end{array}$ & $8: 17$ & 497 & 35 & 28 \\
\hline S4 & QR pendent, above foot bed \#1 & $9: 49$ & 520 & 35 & 28 \\
\hline S11 & QR sidewall, overhead bed $\sharp 2$ & $9: 03$ & 543 & 36 & 28 \\
\hline S4 & QR-EC pendent, center of room & $9: 17$ & 557 & 35 & 28 \\
\hline S2 & QR pendent, above foot bed \#2 & $9: 49$ & 596 & 33 & 28 \\
\hline S8 & standard pendent, above foot bed $\# 1$ & $9: 56$ & 596 & 37 & 39 \\
\hline S7 & QR sidewall, across foot bed $\# 2$ & $10: 32$ & 632 & 39 & 29 \\
\hline S5 & QR sidewall, across foot bed $\# 1$ & $10: 34$ & 634 & 39 & 29 \\
\hline S9 & $\begin{array}{l}\text { QR concealed pendent, above foot bed } \\
\text { \#2 }\end{array}$ & $10: 56$ & 656 & 41 & 29 \\
\hline S1 & QR-EC sidewall, east wall, bathroom & 13.40 & 820 & 59 & 31 \\
\hline $\mathrm{S} 6 \mathrm{n} / \mathrm{a}$ & standard sidewall, across foot bed $\# 2$ & - & - & - & - \\
\hline
\end{tabular}


Table 4 - Activation times, Closed door shielded fire test

\begin{tabular}{|c|c|c|c|c|c|}
\hline $\begin{array}{l}\text { Device } \\
\text { Number }\end{array}$ & Description & $\begin{array}{l}\text { Activation } \\
\text { Time } \\
\text { min: s }\end{array}$ & $\begin{array}{c}\text { Activation } \\
\text { Time } \\
\mathrm{s}\end{array}$ & $\begin{array}{c}1.5 \mathrm{~m} 5(\mathrm{ft}) \\
\text { Elevation } \\
\text { Between } \\
\text { Beds } \\
\text { Temp }^{\circ} \mathrm{C}\end{array}$ & $\begin{array}{c}0.91 \mathrm{~m}(3 \mathrm{ft}) \\
\text { Elevation } \\
\text { Between Beds } \\
\text { Temp }{ }^{\circ} \mathrm{C}\end{array}$ \\
\hline D2 & ion, south wall, between beds & $1: 07$ & 67 & 29 & 29 \\
\hline D3 & ion, ceiling, center of room & $1: 39$ & 99 & 31 & 27 \\
\hline D3 & ion, east wall, bathroom & $2: 20$ & 140 & 30 & 27 \\
\hline D6 & ion, ceiling, near room door & $2: 47$ & 167 & 34 & 27 \\
\hline D1 & photo, south wall, between beds & $5: 01$ & 301 & 42 & 33 \\
\hline D7 & photo ceiling, center of room & $\mathbf{8 : 1 5}$ & 315 & 46 & 34 \\
\hline $\mathrm{DSA}$ & photo sensor, south wall. between beds & $5: 16$ & 316 & 47 & 34 \\
\hline DSD & photo sensor, ceiling, center of room & $5: 20$ & 320 & 48 & 34 \\
\hline DSB & photö sensor, east wall, bathroom & $5: 20$ & 320 & 48 & 34 \\
\hline D3 & photo, east wall, bathroom & $5: 23$ & 323 & 38 & 34 \\
\hline D5 & photo, ceiling, near room door & $5: 53$ & 353 & 53 & 40 \\
\hline DSC & photo sensor, ceiling, near room door & $6: 00$ & 360 & 51 & 38 \\
\hline S12 & QR sidewall, overhead bed \#1 & $6: 06$ & 366 & 53 & 38 \\
\hline S4 & QR pendent, above foot bed \#1 & $6: 18$ & 378 & 57 & 38 \\
\hline S4 & QR-EC pendent, center of room & $8: 15$ & 495 & 53 & 53 \\
\hline S8 & standard pendent, above foot bed $\# 1$ & $6: 00$ & 520 & 72 & 59 \\
\hline S7 & QR sidewall, across foot bed $\sharp 2$ & $8: 44$ & 524 & 71 & 54 \\
\hline$\$ 10$ & $\begin{array}{l}\text { QR concealed pendent, above foot bed } \\
\# 1\end{array}$ & $8: 56$ & 536 & 72 & 58 \\
\hline S11 & QR sidewall, overhead bed $\# 2$ & $8: 56$ & 548 & 72 & 58 \\
\hline S2 & QR pendent, above foot bed $\sharp 2$ & 9:04 & 544 & 74 & 59 \\
\hline S5 & QR sidewall, across foot bed \#1 & 9:08 & 548 & 47 & 59 \\
\hline S9 & $\begin{array}{l}\text { QR concealed pendent, above foot bed } \\
\# 2\end{array}$ & $9: 59$ & 599 & 89 & 66 \\
\hline S6 & standard sidewall, across foot bed $\# 2$ & $11: 15$ & 675 & 108 & 75 \\
\hline $\mathrm{S} 1 \mathrm{n} / \mathrm{a}$ & QR-EC sidewall, east wall, bathroom & $11: 40$ & 700 & 111 & 78 \\
\hline
\end{tabular}




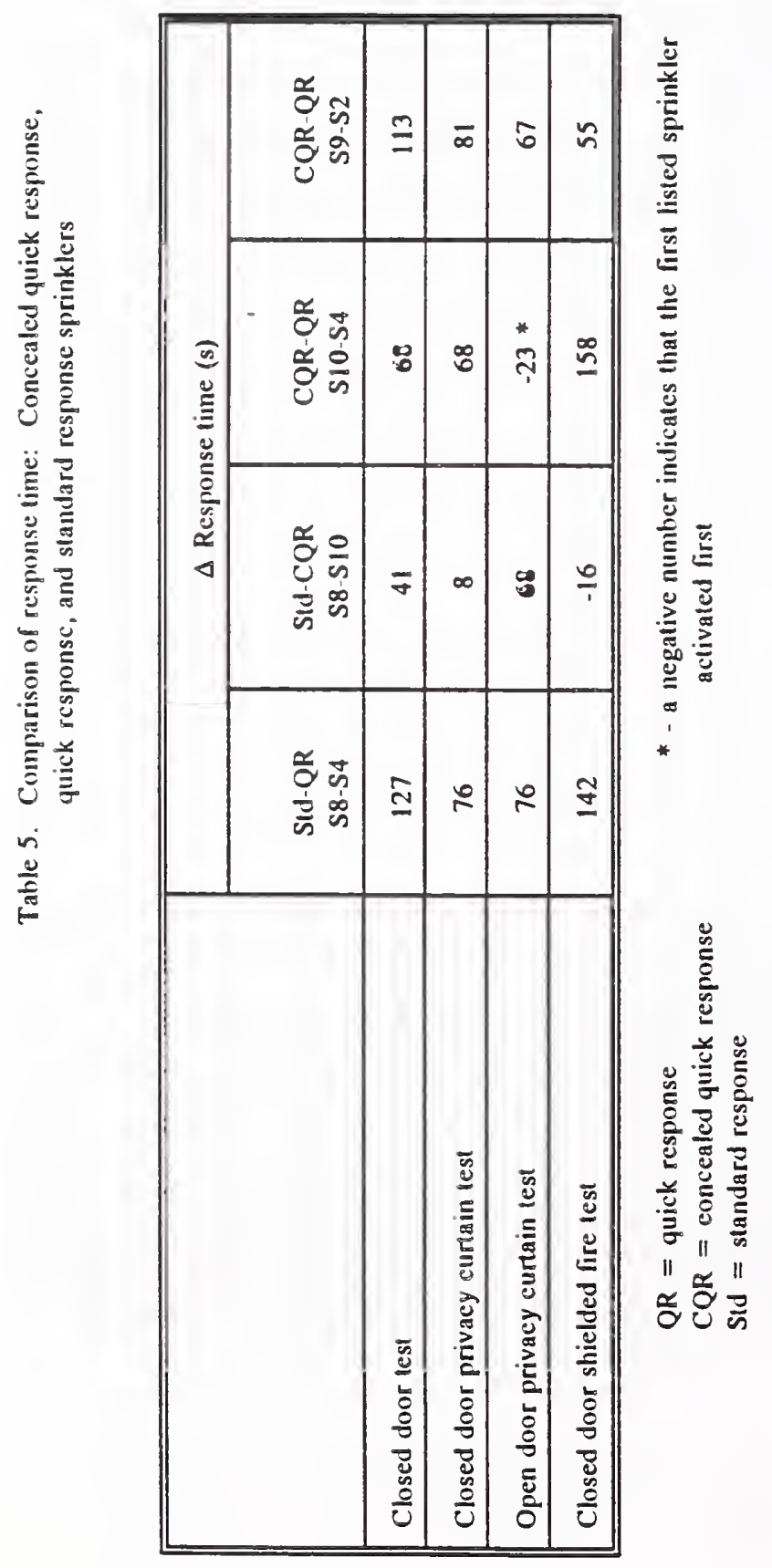




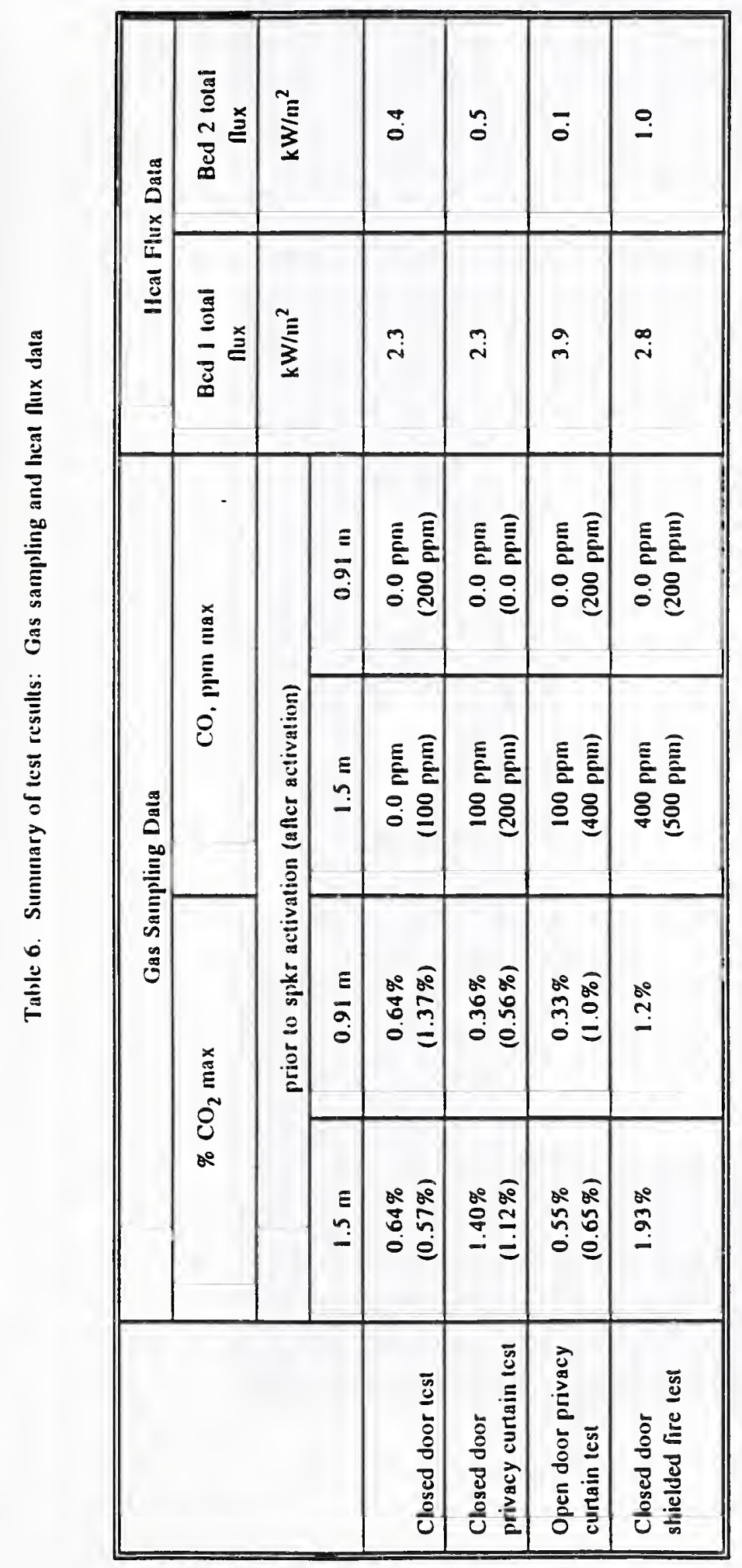




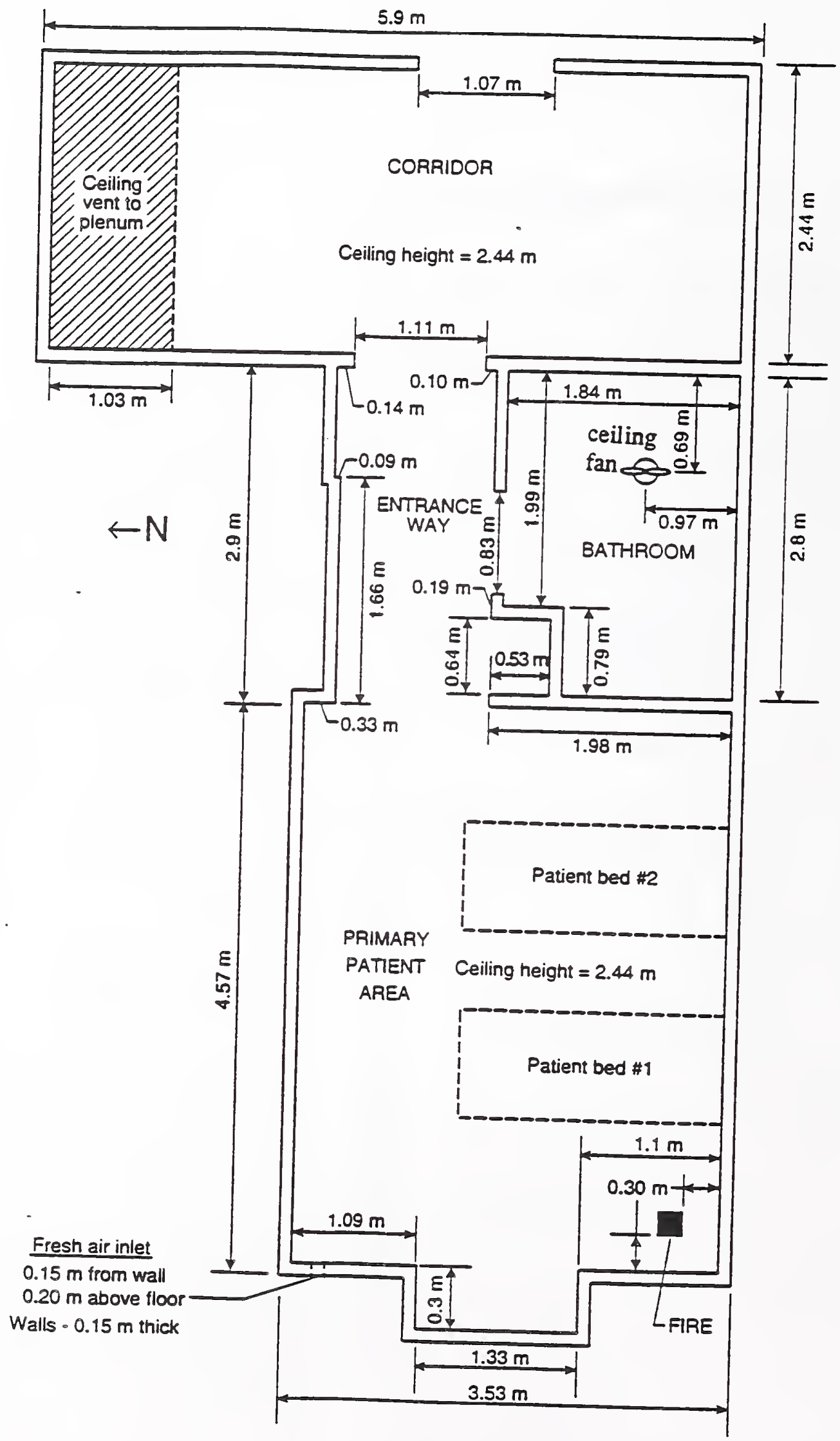

Figure 1. Plan view of simulated hospital patient room and corridor 


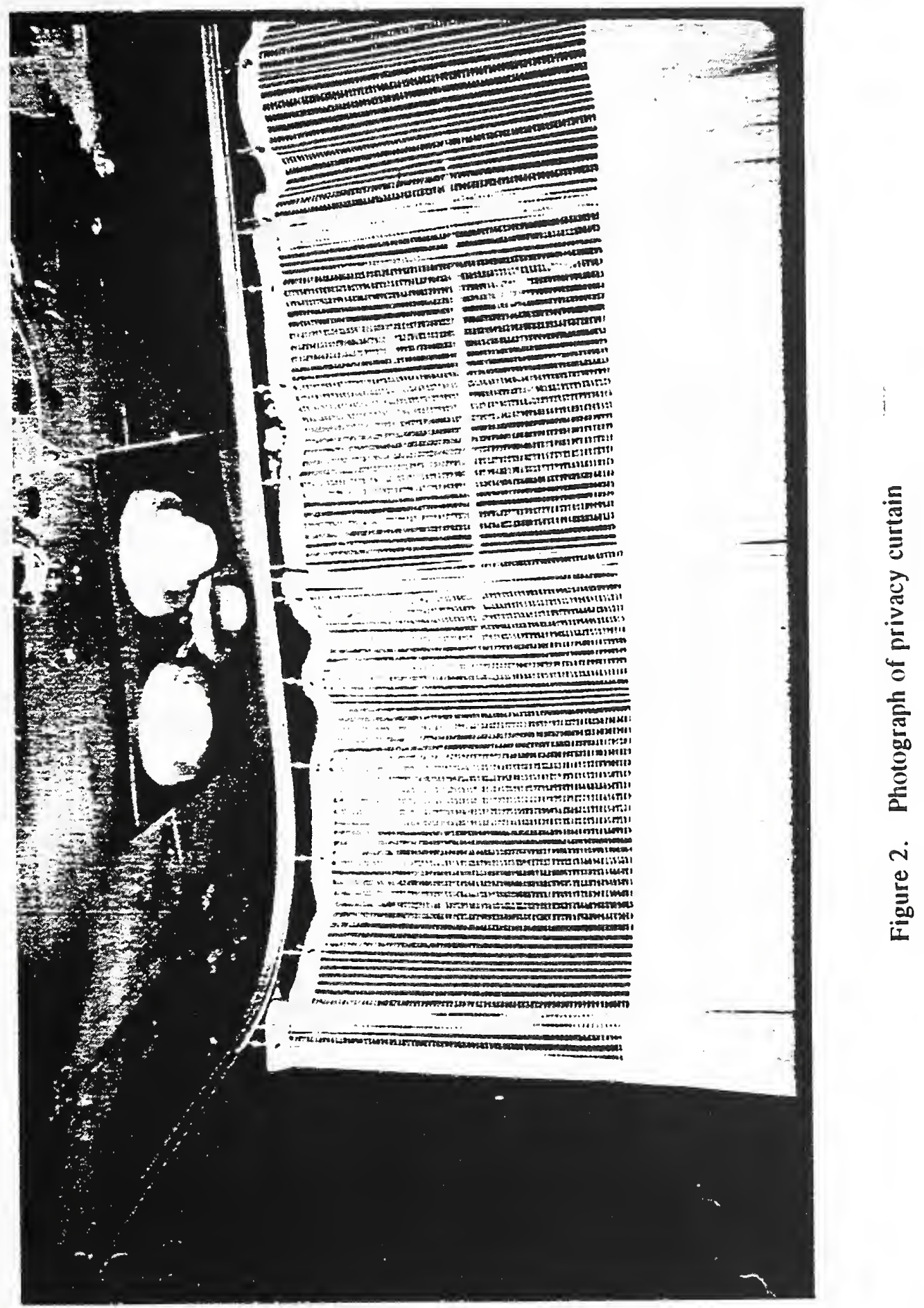




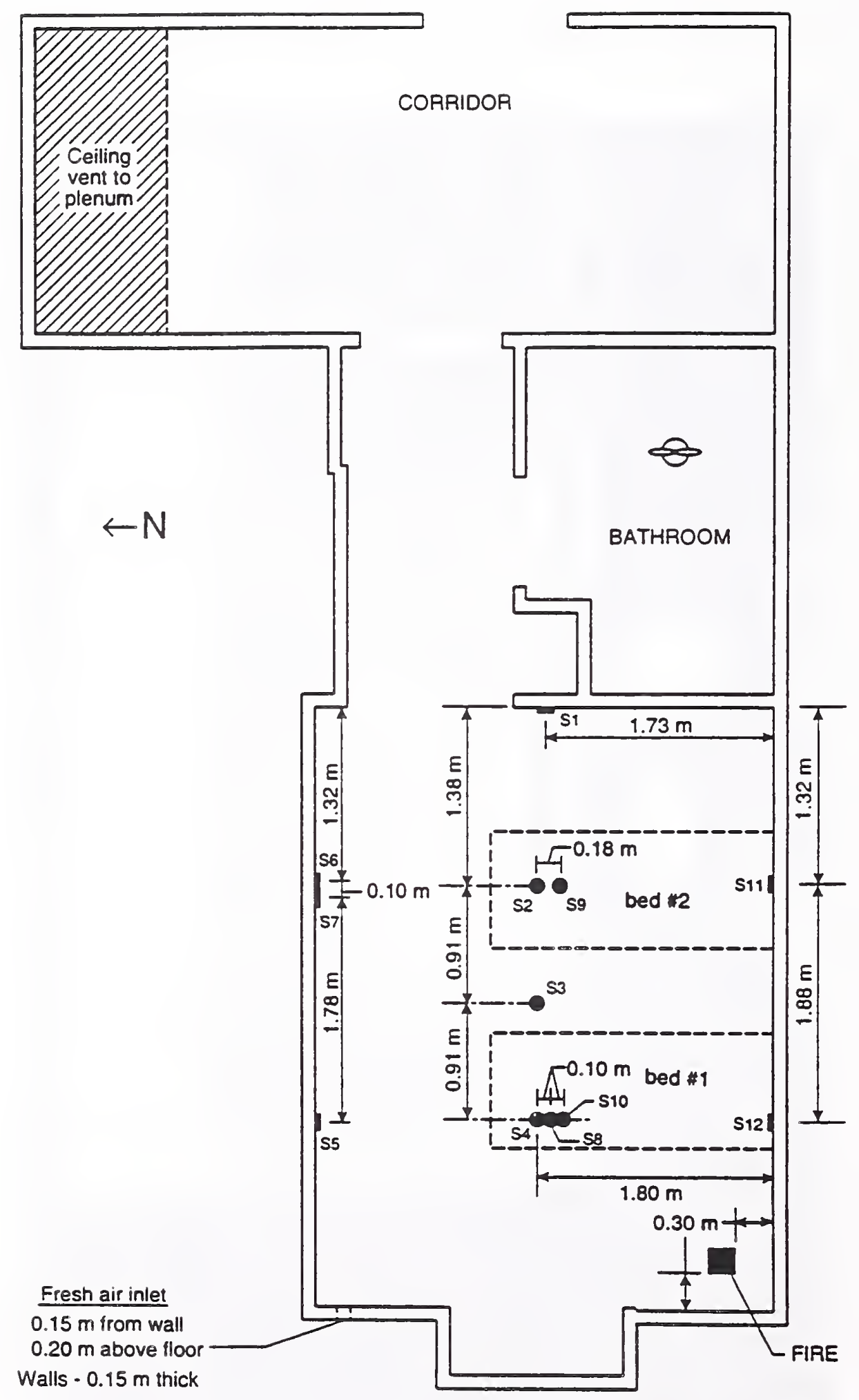

Figure 3. Locations of automatic sprinklers 


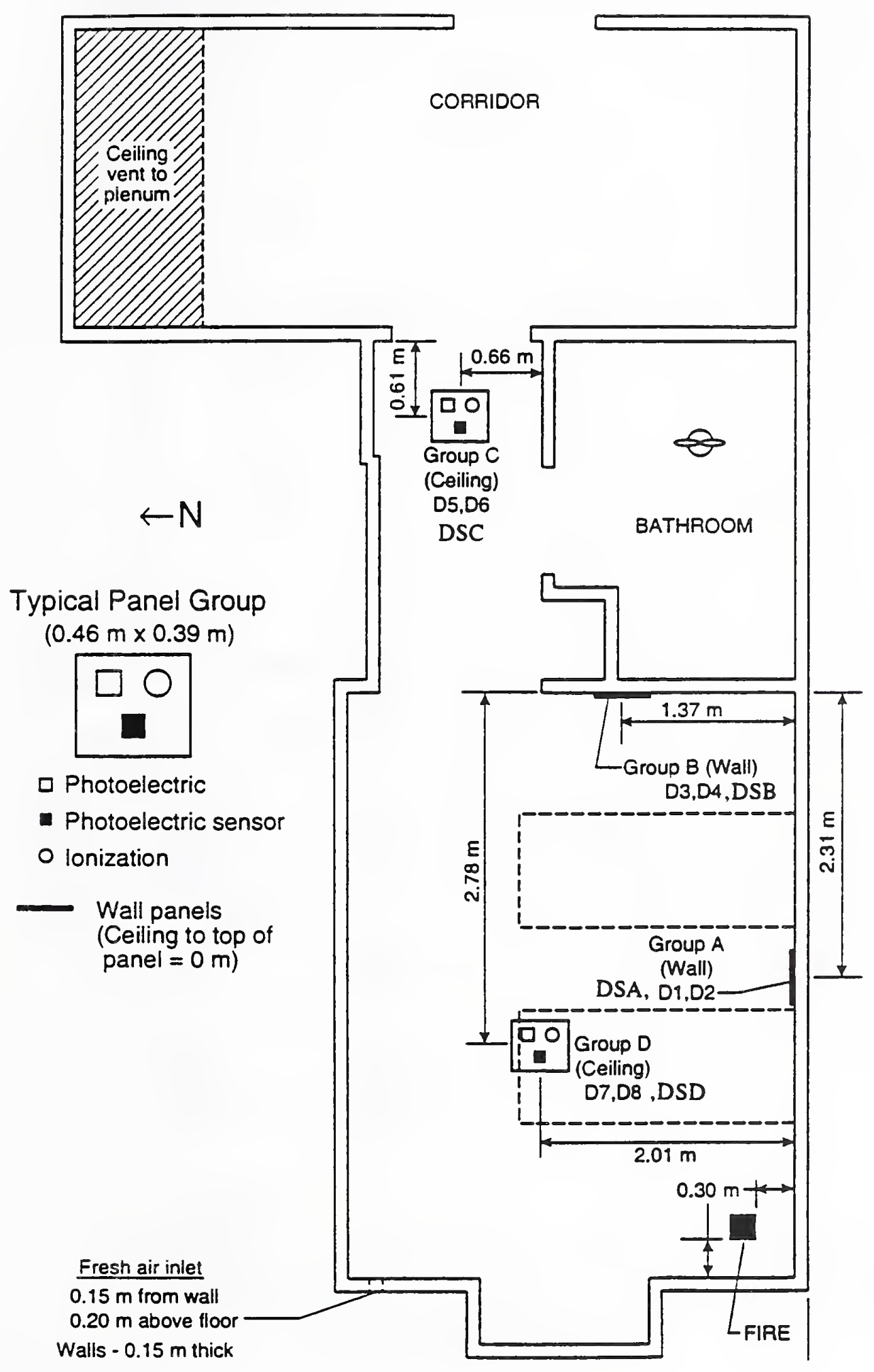

Figure 4. Locations of smoke detectors 


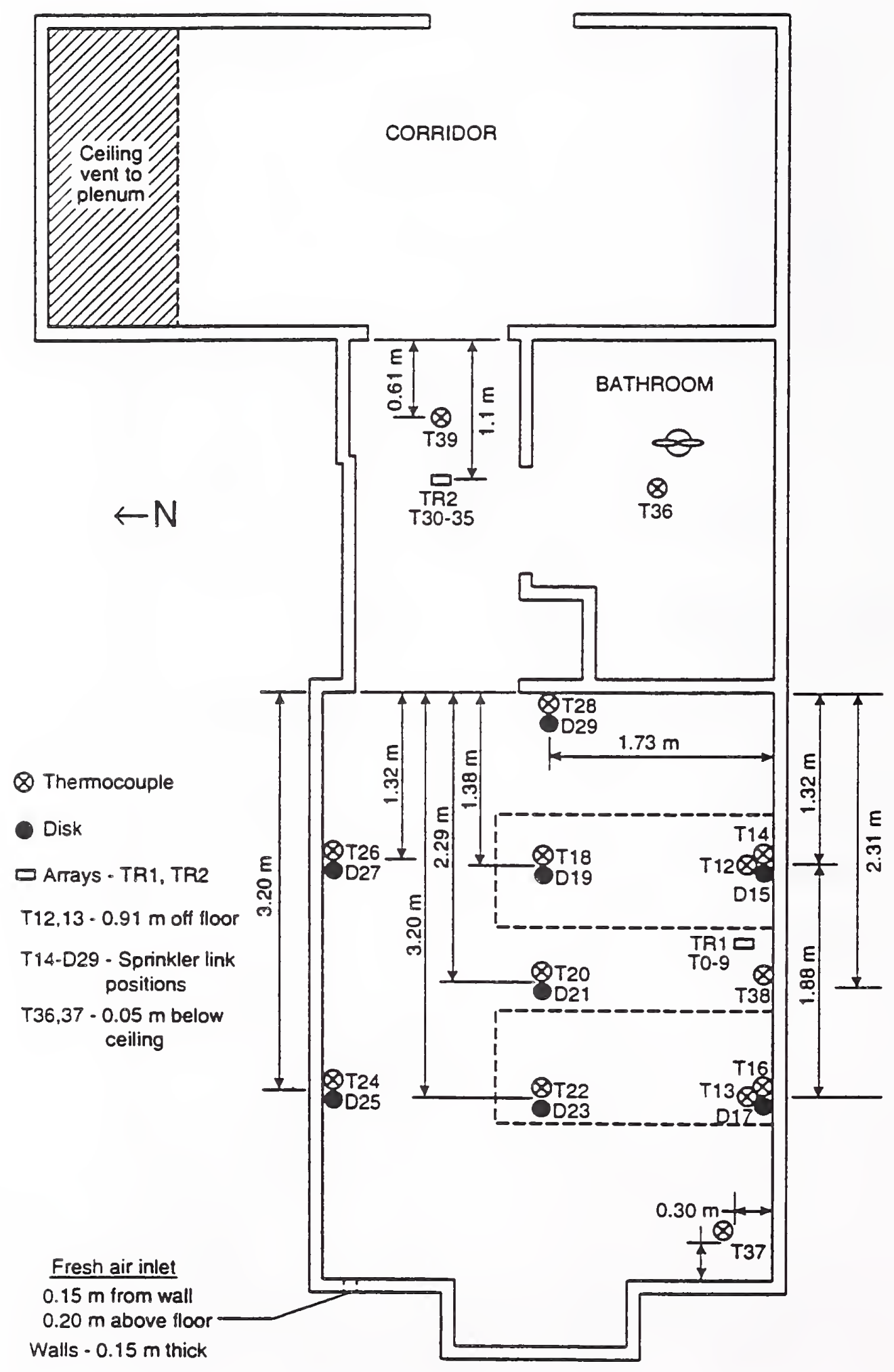

Figure 5. Thermocouple and disk locations 


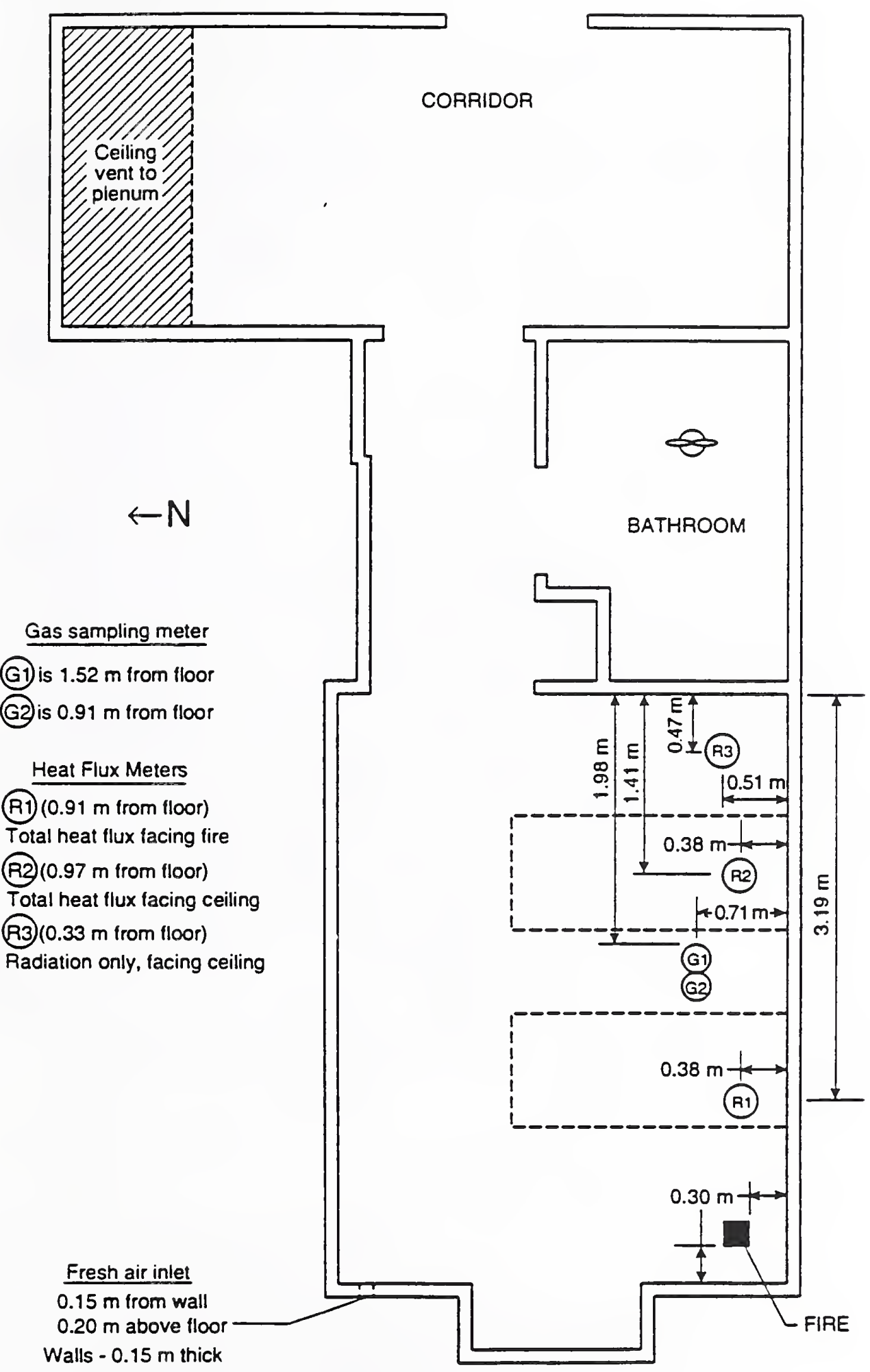

Figure 6. Gas sampling and heat flux meter locations 


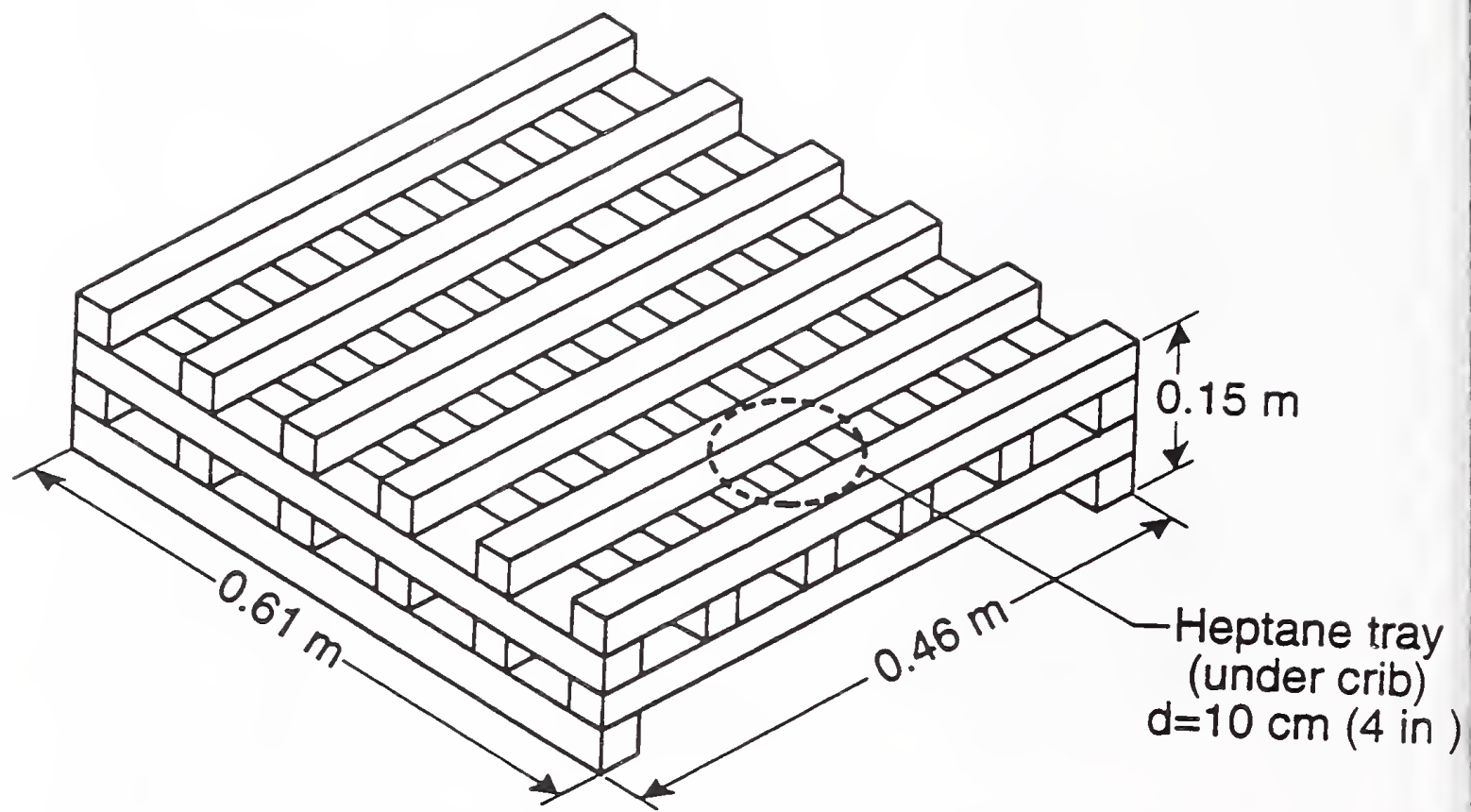

Figure 7. Test fuel source - wood crib 


\section{FLAMING WOOD CRIB FIRES}

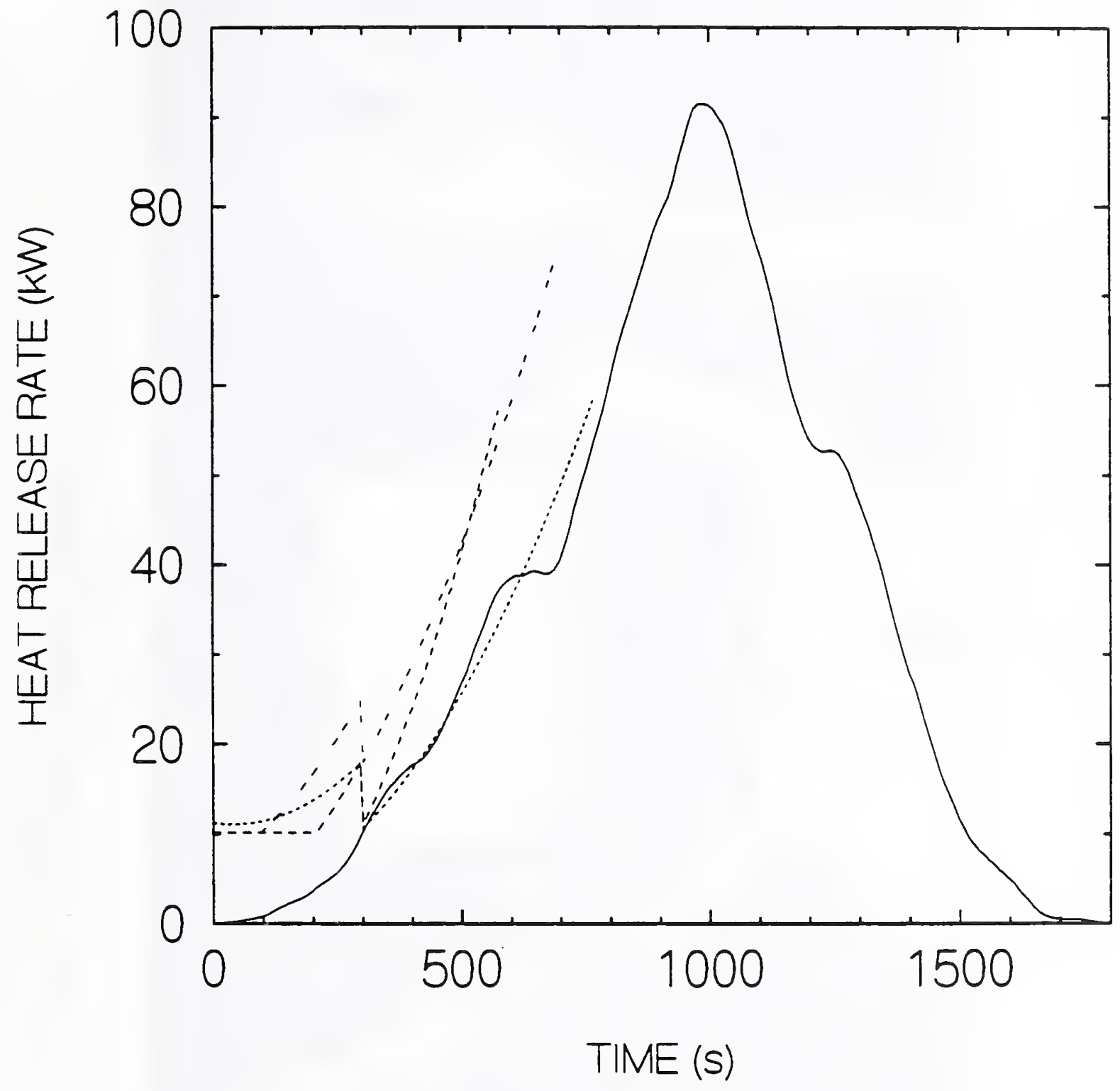

Freeburn (measured)

Closed door test (calculated)

Closed door privacy curtain test (calculated)

-. . . - . - Open door privacy curtain test (calculated)

Figure 8. Heat release rate curves 


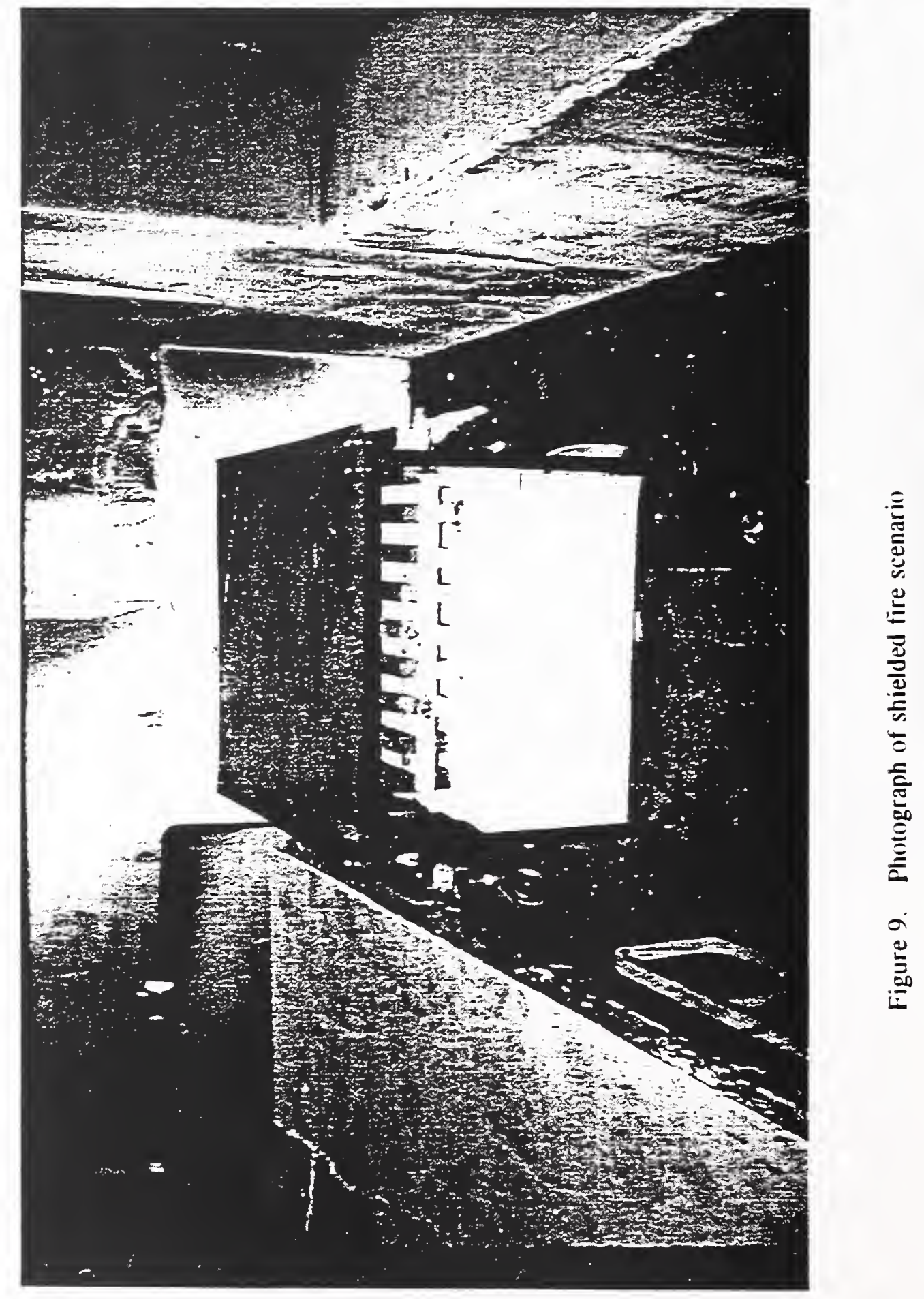




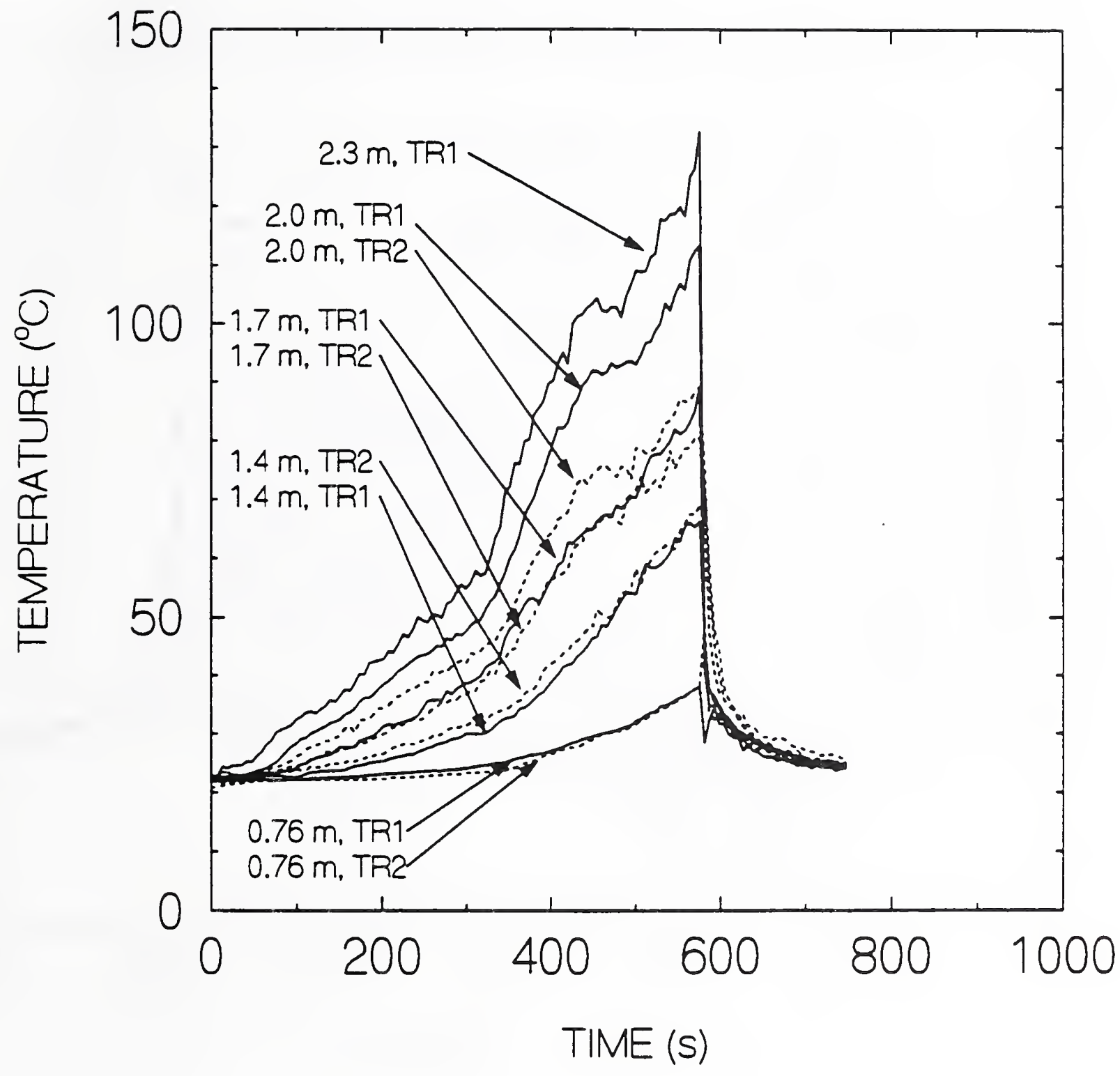

- TR1 - Thermocouple array between beds

-------- TR2 - Thermocouple array room entrance way

Figure 10. Temperatures in room, closed door test 


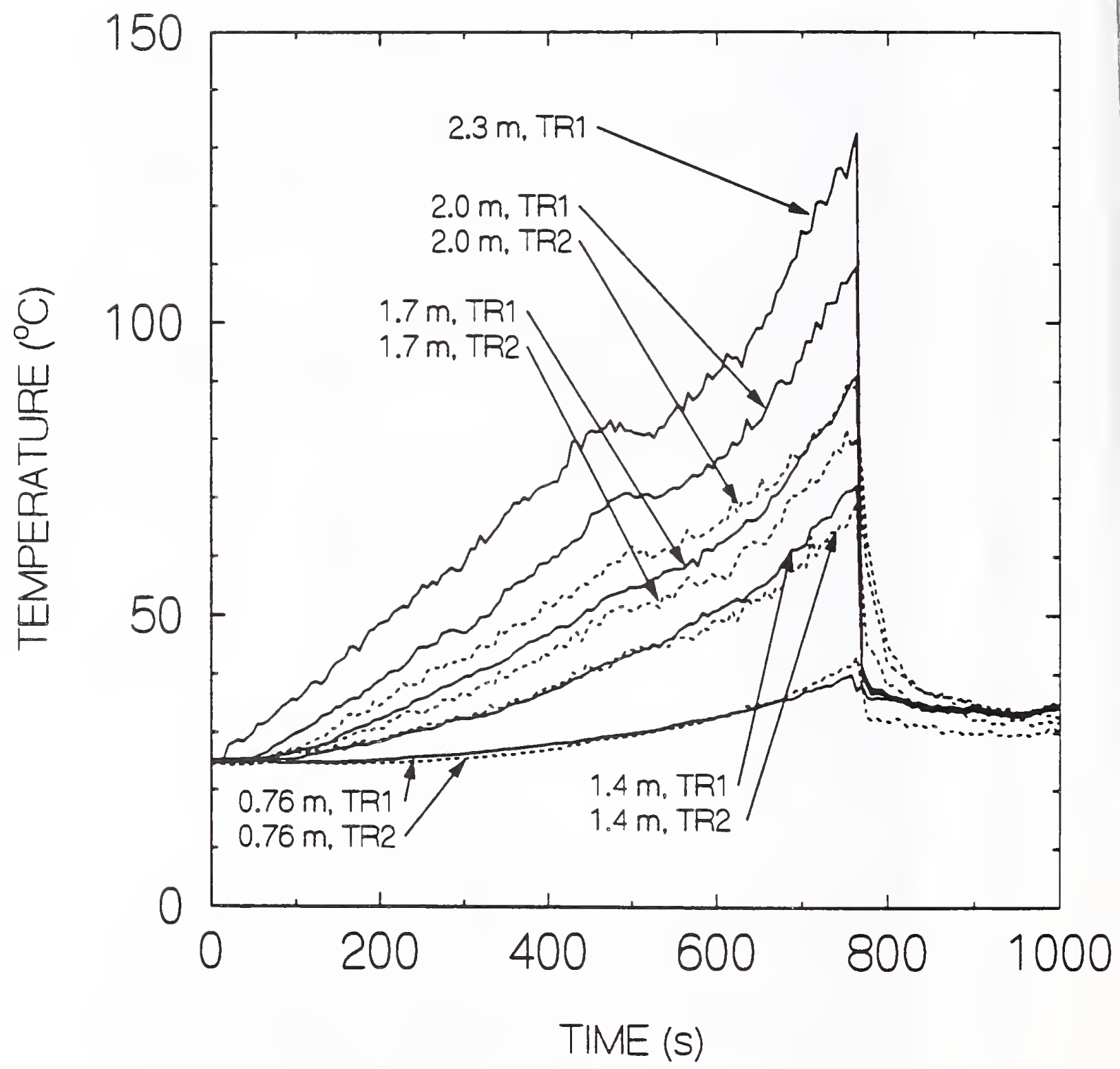

- TR1 - Thermocouple array between beds

-.---.-- TR2 - Thermocouple array room entrance way

Figure 11. Temperatures in room, closed door privacy curtain test 


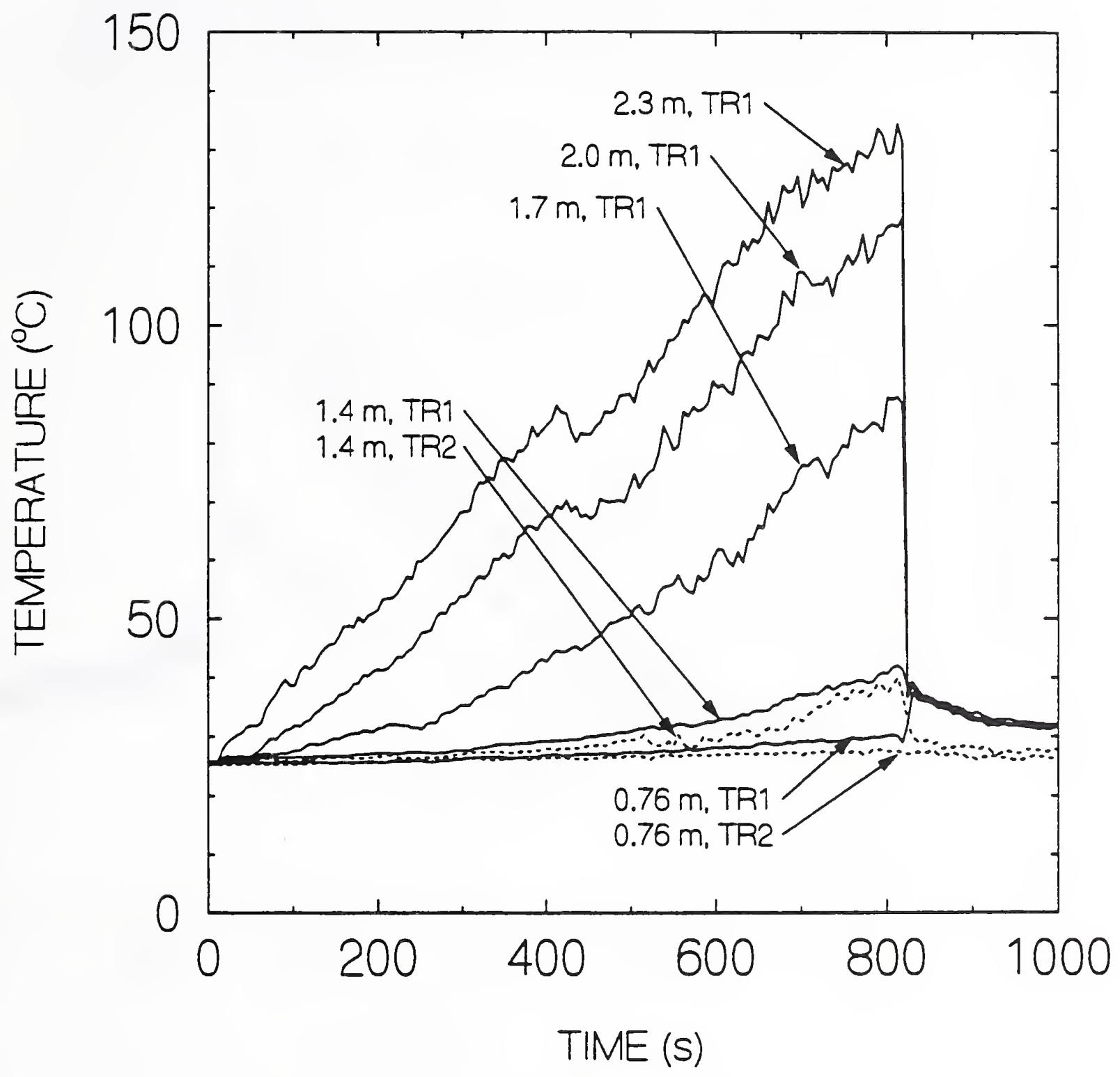

_ TR1 - Thermocouple array between beds

-----.-- TR2 - Thermocouple array room entrance way

Figure 12. Temperatures in room, open door privacy curtain test 


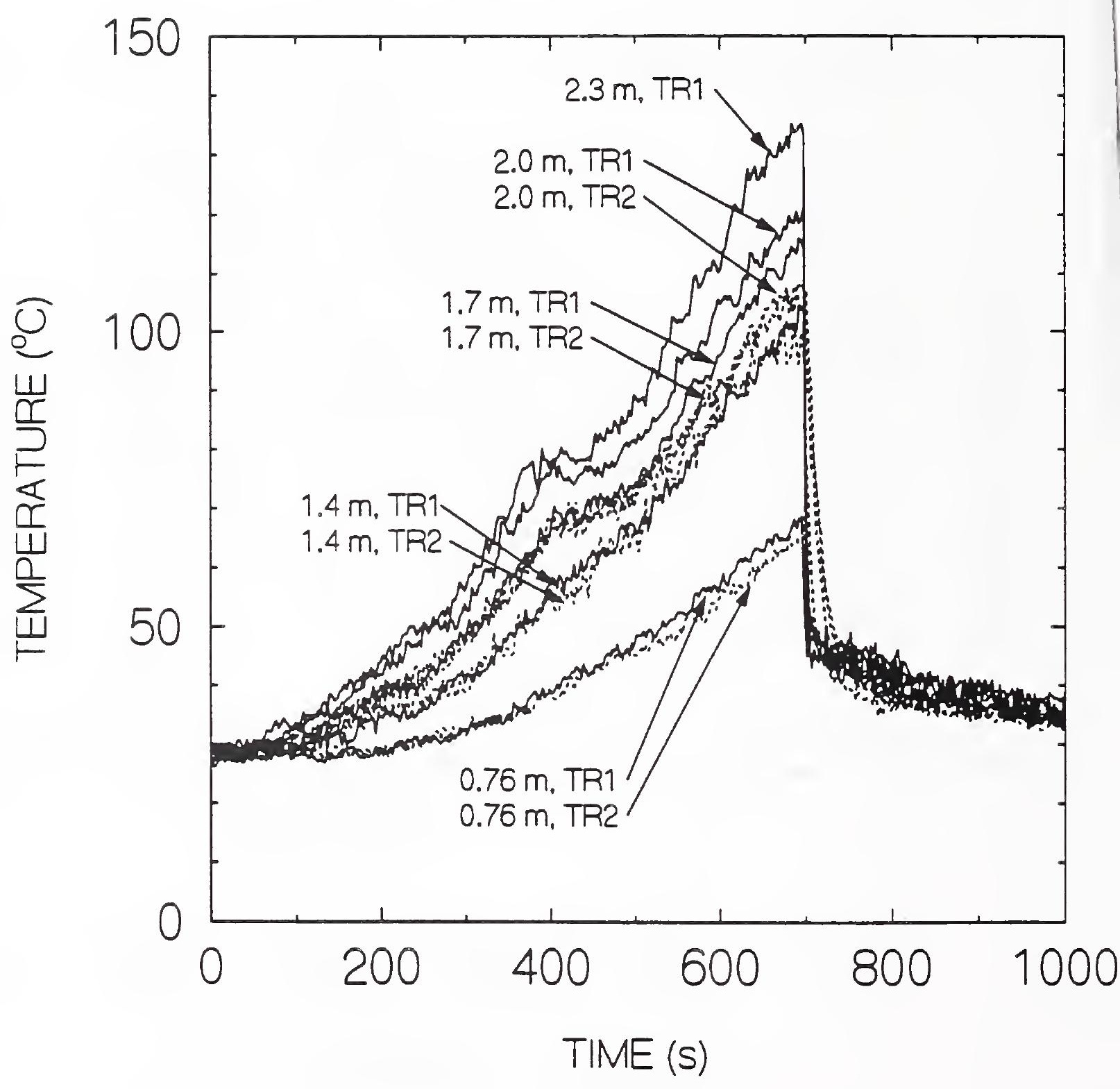

- TR1 - Thermocouple array between beds

.......- TR2 - Thermocouple array room entrance way

Figure 13. Temperatures in room, closed door shielded fire test 


\subsection{APPENDIX}

The Appendix contains time/temperature data at sprinkler locations from preignition to completion of the test. Numbering of thermocouples and disks at sprinkler locations follows the nomenclature of figure 5. Also included are the time/temperature data at the four smoke detector groups shown in figure 4. Smoke detector groups A - D are denoted PAN A - PAN D in the Appendix respectively. 

CLOSED DOOR TEST

THERMOCOUPLES AND DISKS AT SPRINKLER AND DETECTOR LOCATIONS - ${ }^{\circ} \mathrm{C}$

\begin{tabular}{|c|c|c|c|c|c|c|c|c|c|c|}
\hline TIME & $\mathrm{T} 14$ & D15 & $\mathrm{T} 16$ & D17 & T18 & D19 & T20 & $\mathrm{D} 21$ & T22 & $\mathrm{D} 23$ \\
\hline-45 & 22 & 22 & 22 & 22 & 22 & 22 & 21 & 22 & 22 & 22 \\
\hline-38 & 22 & 22 & 22 & 22 & 23 & 22 & 22 & 22 & 22 & 22 \\
\hline-32 & 22 & 22 & 22 & 22 & 23 & 22 & 22 & 22 & 22 & 22 \\
\hline-26 & 22 & 22 & 22 & 22 & 22 & 22 & 22 & 22 & 22 & 22 \\
\hline-20 & 22 & 22 & 22 & 22 & 23 & 22 & 22 & 22 & 22 & 22 \\
\hline-15 & 22 & 22 & 22 & 22 & 23 & 22 & 22 & 22 & 22 & 22 \\
\hline-9 & 22 & 22 & 22 & 22 & 23 & 22 & 22 & 22 & 22 & 22 \\
\hline-3 & 22 & 22 & 22 & 22 & 23 & 22 & 22 & 22 & 22 & 22 \\
\hline 1 & 22 & 22 & 22 & 22 & 23 & 22 & 22 & 22 & 22 & 22 \\
\hline 7 & 22 & 22 & 24 & 23 & 23 & 22 & 22 & 22 & 22 & 22 \\
\hline 13 & 22 & 22 & 24 & 23 & 23 & 22 & 22 & 22 & 24 & 22 \\
\hline 19 & 23 & 22 & 25 & 24 & 23 & 22 & 23 & 22 & 24 & 23 \\
\hline 24 & 24 & 23 & 26 & 24 & 23 & 23 & 23 & 23 & 24 & 23 \\
\hline 30 & 24 & 23 & 27 & 25 & 23 & 23 & 25 & 23 & 25 & 23 \\
\hline 36 & 25 & 23 & 28 & 26 & 24 & 23 & 26 & 23 & 26 & 23 \\
\hline 42 & 25 & 23 & 29 & 26 & 25 & 23 & 26 & 24 & 26 & 24 \\
\hline 47 & 25 & 23 & 30 & 27 & 26 & 24 & 27 & 24 & 27 & 25 \\
\hline 53 & 25 & 24 & 31 & 28 & 26 & 24 & 27 & 25 & 27 & 25 \\
\hline 59 & 27 & 24 & 32 & 29 & 27 & 25 & 29 & 25 & 29 & 26 \\
\hline 65 & 28 & 25 & 34 & 30 & 28 & 25 & 30 & 26 & 30 & 26 \\
\hline 70 & 28 & 25 & 34 & 31 & 29 & 26 & 31 & 27 & 29 & 27 \\
\hline 76 & 29 & 26 & 35 & 32 & 30 & 27 & 31 & 27 & 31 & 28 \\
\hline 82 & 29 & 26 & 36 & 33 & 30 & 27 & 32 & 28 & 32 & 28 \\
\hline 87 & 31 & 27 & 37 & 34 & 31 & 28 & 33 & 29 & 32 & 29 \\
\hline 93 & 31 & 27 & 37 & 35 & 31 & 28 & 33 & 29 & 33 & 30 \\
\hline
\end{tabular}


THERMOCOUPLES AND DISKS AT SPRINKLER AND DETECTOR LOCATIONS - ${ }^{\circ} \mathrm{C}$

\begin{tabular}{|c|c|c|c|c|c|c|c|c|c|c|}
\hline TIME & $T_{14}$ & D15 & T16 & D17 & T18 & D19 & T20 & D21 & T22 & D23 \\
\hline 99 & 31 & 28 & 38 & 35 & 31 & 29 & 33 & 30 & . 34 & 30 \\
\hline 105 & 32 & 29 & 39 & 36 & 32 & 29 & 34 & 30 & 34 & 31 \\
\hline 110 & 33 & 29 & 39 & 37 & 32 & 30 & 34 & 31 & 35 & 32 \\
\hline 116 & 33 & 30 & 41 & 38 & 32 & 30 & 35 & 31 & 35 & 32 \\
\hline 122 & 34 & 30 & 40 & 38 & 33 & 31 & 35 & 32 & 36 & 33 \\
\hline 128 & 34 & 31 & 41 & 39 & 33 & 31 & 36 & 32 & 34 & 33 \\
\hline 133 & 34 & 31 & 42 & 40 & 34 & 32 & 36 & 33 & 35 & 34 \\
\hline 939 & 35 & 32 & 42 & 40 & 36 & 32 & 37 & 34 & 36 & 34 \\
\hline 145 & 35 & 32 & 43 & 41 & 35 & 33 & 37 & 34 & 37 & 35 \\
\hline 151 & 36 & 33 & 44 & 42 & 36 & 33 & 38 & 35 & 38 & 35 \\
\hline 156 & 37 & 33 & 46 & 43 & 36 & 34 & 40 & 35 & 39 & 36 \\
\hline 162 & 37 & 34 & 46 & 43 & 37 & 34 & 41 & 36 & 42 & 37 \\
\hline 168 & 38 & 34 & 47 & 44 & 38 & 35 & 41 & 37 & 39 & 37 \\
\hline 173 & 39 & 35 & 48 & 45 & 38 & 35 & 41 & 37 & 42 & 38 \\
\hline 179 & 40 & 36 & 50 & 46 & 39 & 36 & 42 & 38 & 42 & 39 \\
\hline 185 & 41 & 37 & 50 & 47 & 40 & 37 & 43 & 39 & 45 & 40 \\
\hline 191 & 41 & 37 & 51 & 48 & 40 & 37 & 44 & 39 & 44 & 41 \\
\hline 196 & 42 & 38 & 52 & 49 & 40 & 38 & 44 & 40 & 44 & 42 \\
\hline 202 & 42 & 38 & 52 & 50 & 42 & 38 & 44 & 41 & 47 & 43 \\
\hline 208 & 43 & 39 & 53 & 51 & 43 & 39 & 46 & 41 & 45 & 43 \\
\hline 213 & 44 & 40 & 54 & 51 & 43 & 40 & 49 & 42 & 48 & 44 \\
\hline 219 & 43 & 40 & 56 & 53 & 44 & 41 & 48 & 43 & 48 & 45 \\
\hline 225 & 45 & 41 & 57 & 54 & 45 & 41 & 47 & 44 & 50 & 46 \\
\hline 231 & 45 & 42 & 56 & 54 & 45 & 42 & 49 & 44 & 51 & 47 \\
\hline 237 & 47 & 42 & 59 & 55 & 45 & 42 & 49 & 45 & 51 & 48 \\
\hline
\end{tabular}


THERMOCOUPLES AND DISKS AT SPRINKLER AND DETECTOR LOCATIONS - ${ }^{\circ} \mathrm{C}$

\begin{tabular}{|c|c|c|c|c|c|c|c|c|c|c|}
\hline TIME & T14 & D15 & T16 & D17 & T18 & D19 & T20 & D21 & T22 & D23 \\
\hline 242 & 48 & 43 & 59 & 56 & 45 & 43 & 49 & 46 & 53 & 49 \\
\hline 248 & 48 & 44 & 59 & 57 & 45 & 43 & 52 & 46 & 54 & 50 \\
\hline 254 & 48 & 44 & 61 & 58 & 45 & 44 & 52 & 47 & 51 & 50 \\
\hline 259 & 49 & 45 & 62 & 59 & 48 & 45 & 51 & 47 & 53 & 50 \\
\hline 265 & 48 & 45 & 62 & 59 & 48 & 45 & 52 & 48 & 54 & 51 \\
\hline 271 & 50 & 46 & 64 & 60 & 48 & 46 & 53 & 49 & 55 & 52 \\
\hline 277 & 52 & 47 & 63 & 61 & 49 & 46 & 53 & 49 & 54 & 52 \\
\hline 282 & 52 & 48 & 65 & 62 & 49 & 47 & 54 & 50 & 55 & 53 \\
\hline 288 & 51 & 48 & 67 & 63 & 50 & 47 & 55 & 50 & 56 & 53 \\
\hline 294 & 53 & 49 & 69 & 64 & 50 & 48 & 57 & 51 & 58 & 55 \\
\hline 299 & 53 & 49 & 69 & 65 & 51 & 48 & 55 & 52 & 56 & 55 \\
\hline 305 & 55 & 50 & 72 & 67 & 52 & 49 & 60 & 53 & 59 & 56 \\
\hline 311 & 56 & 51 & 74 & 68 & 55 & 50 & 59 & 54 & 61 & 57 \\
\hline 317 & 57 & 52 & 74 & 69 & 55 & 51 & 60 & 54 & 65 & 59 \\
\hline 322 & 58 & 53 & 78 & 71 & 56 & 52 & 61 & 55 & 63 & 60 \\
\hline 328 & 61 & 54 & 78 & 73 & 59 & 53 & 67 & 57 & 69 & 62 \\
\hline 334 & 63 & 56 & 83 & 75 & 60 & 55 & 70 & 59 & 72 & 64 \\
\hline 340 & 67 & 57 & 86 & 78 & 63 & 56 & 70 & 61 & 73 & 66 \\
\hline 345 & 69 & 59 & 90 & 82 & 65 & 58 & 72 & 63 & $\pi$ & 69 \\
\hline 351 & 72 & 61 & 97 & 86 & 66 & 60 & 74 & 65 & 83 & 72 \\
\hline 357 & 73 & 63 & 96 & 89 & 70 & 62 & 78 & 67 & 82 & 74 \\
\hline 363 & 75 & 65 & 99 & 91 & 74 & 64 & 80 & 69 & 84 & 76 \\
\hline 368 & 78 & 68 & 104 & 95 & 73 & 66 & 79 & 70 & 85 & 78 \\
\hline 374 & 77 & 69 & 106 & 97 & 75 & 68 & 83 & 72 & 89 & 81 \\
\hline 380 & 82 & 72 & 105 & 99 & 78 & 70 & 84 & 74 & 93 & 83 \\
\hline
\end{tabular}


THERMOCOUPLES AND DISKS AT SPRINKLER AND DETECTOR LOCATIONS - ${ }^{\circ} \mathrm{C}$

\begin{tabular}{|c|c|c|c|c|c|c|c|c|c|c|}
\hline TIME & T14 & D15 & $T_{16}$ & D17 & T18 & D19 & T20 & D21 & T22 & $\mathrm{D} 23$ \\
\hline 385 & 83 & 73 & 110 & 102 & 75 & 71 & 89 & 76 & 95 & 86 \\
\hline 391 & 87 & 76 & 111 & 103 & 76 & 73 & 88 & 77 & 92 & 87 \\
\hline 397 & 90 & 78 & 911 & 105 & 78 & 74 & 88 & 79 & 94 & 89 \\
\hline 403 & 89 & 80 & 116 & 107 & 81 & 76 & 91 & 81 & 97 & 91 \\
\hline 408 & 92 & 82 & 118 & 109 & 84 & 78 & 96 & 83 & 102 & 93 \\
\hline 414 & 93 & 84 & 121 & 112 & 84 & 79 & 96 & 85 & 100 & 95 \\
\hline 420 & 92 & 85 & 120 & 113 & 86 & 81 & 97 & 87 & 102 & 96 \\
\hline 426 & 96 & 87 & 120 & 115 & 88 & 83 & 98 & 88 & 104 & 98 \\
\hline 431 & 96 & 89 & 123 & 116 & 87 & 85 & 101 & 90 & 102 & 99 \\
\hline 437 & 99 & 90 & 125 & 918 & 88 & 86 & 98 & 91 & 104 & 101 \\
\hline 443 & 100 & 92 & 126 & 120 & 90 & 87 & 97 & 91 & 105 & 102 \\
\hline 448 & 99 & 93 & 121 & 118 & 85 & 87 & 100 & 92 & 104 & 103 \\
\hline 454 & 99 & 93 & 117 & 117 & 87 & 87 & 97 & 93 & 104 & 103 \\
\hline 460 & 100 & 94 & 121 & 118 & 90 & 88 & 99 & 93 & 102 & 103 \\
\hline 466 & 98 & 94 & 123 & 118 & 88 & 87 & 97 & 93 & 100 & 102 \\
\hline 471 & 99 & 95 & 119 & 118 & 91 & 88 & 99 & 93 & 103 & 103 \\
\hline 477 & 99 & 95 & 121 & 117 & 88 & 88 & 101 & 94 & 108 & 104 \\
\hline 483 & 101 & 96 & 125 & 118 & 91 & 88 & 101 & 95 & 106 & 105 \\
\hline 489 & 103 & 97 & 128 & 120 & 91 & 88 & 103 & 95 & 108 & 106 \\
\hline 494 & 104 & 98 & 131 & 122 & 93 & 89 & 102 & 96 & 110 & 107 \\
\hline 500 & 106 & 99 & 131 & 124 & 92 & 89 & 100 & 96 & 109 & 107 \\
\hline 506 & 108 & 101 & 133 & 126 & 94 & 90 & 104 & 97 & 110 & 108 \\
\hline 512 & 107 & 102 & 137 & 128 & 97 & 91 & 112 & 99 & 118 & 111 \\
\hline 517 & 110 & 103 & 135 & 129 & 97 & 92 & 111 & 101 & 198 & 112 \\
\hline 523 & 115 & 105 & 137 & 131 & 99 & 93 & 111 & 102 & 118 & 113 \\
\hline
\end{tabular}


THERMOCOUPLES AND DISKS AT SPRINKLER AND DETECTOR LOCATIONS - ${ }^{\circ} \mathrm{C}$

\begin{tabular}{|c|c|c|c|c|c|c|c|c|c|c|}
\hline TIME & T14 & D15 & T16 & D17 & T18 & D19 & T20 & D21 & T22 & D23 \\
\hline 529 & 113 & 106 & 143 & 133 & 100 & 94 & 113 & 104 & 125 & 115 \\
\hline 535 & 117 & 107 & 146 & 136 & 107 & 97 & 115 & 105 & 121 & 117 \\
\hline 540 & 118 & 109 & 147 & 138 & 106 & 98 & 118 & 107 & 122 & 119 \\
\hline 546 & 117 & 110 & 146 & 140 & 106 & 99 & 118 & 109 & 132 & 122 \\
\hline 552 & 119 & 111 & 152 & 142 & 108 & 101 & 120 & 110 & 132 & 124 \\
\hline 557 & 120 & 113 & 149 & 143 & 109 & 102 & 124 & 112 & 129 & 125 \\
\hline 563 & 123 & 114 & 153 & 145 & 107 & 103 & 122 & 113 & 133 & 126 \\
\hline 569 & 123 & 115 & 155 & 147 & 109 & 104 & 124 & 115 & 134 & 128 \\
\hline 575 & 127 & 117 & 159 & 149 & 111 & 105 & 125 & 116 & 137 & 130 \\
\hline 580 & 49 & 80 & 45 & 81 & 33 & 32 & 35 & 44 & 111 & 125 \\
\hline 586 & 37 & 46 & 40 & 45 & 36 & 32 & 42 & 45 & 83 & 113 \\
\hline 592 & 35 & 38 & 37 & 41 & 34 & 32 & 42 & 45 & 80 & 104 \\
\hline 598 & 32 & 34 & 33 & 36 & 31 & 30 & 42 & 45 & 69 & 95 \\
\hline 603 & 32 & 32 & 32 & 33 & 31 & 29 & 40 & 43 & 64 & 88 \\
\hline 609 & 31 & 31 & 32 & 32 & 29 & 28 & 41 & 41 & 55 & 80 \\
\hline 615 & 29 & 30 & 30 & 30 & 30 & 28 & 47 & 40 & 52 & 73 \\
\hline 620 & 29 & 29 & 29 & 30 & 28 & 27 & 44 & 39 & 53 & 68 \\
\hline 626 & 28 & 29 & 30 & 30 & 28 & 27 & 46 & 38 & 50 & 64 \\
\hline 632 & 27 & 28 & 29 & 30 & 27 & 26 & 42 & 37 & 47 & 60 \\
\hline 638 & 27 & 28 & 28 & 29 & 26 & 25 & 44 & 36 & 50 & 58 \\
\hline 643 & 27 & 27 & 27 & 28 & 27 & 25 & 42 & 35 & 45 & 55 \\
\hline 649 & 26 & 27 & 27 & 28 & 26 & 25 & 42 & 35 & 44 & 52 \\
\hline 655 & 26 & 27 & 27 & 27 & 25 & 25 & 39 & 34 & 42 & 50 \\
\hline 661 & 26 & 26 & 26 & 27 & 25 & 24 & 34 & 33 & 44 & 49 \\
\hline 666 & 25 & 26 & 26 & 26 & 26 & 24 & 33 & 32 & 40 & 47 \\
\hline
\end{tabular}


CLOSED DOOR TEST

THERMOCOUPLES AND DISKS AT SPRINKLER AND DETECTOR LOCATIONS - ${ }^{\circ} \mathrm{C}$

\begin{tabular}{|c|c|c|c|c|c|c|c|c|c|c|}
\hline TME & T14 & D15 & T16 & 017 & T18 & D19 & T20 & $\mathrm{D} 21$ & T22 & $\mathrm{D} 23$ \\
\hline 672 & 25 & 26 & 26 & 26 & 25 & 24 & 32 & 31 & 40 & 45 \\
\hline 678 & 25 & 26 & 25 & 26 & 25 & 24 & 34 & 31 & 39 & 44 \\
\hline 683 & 25 & 25 & 27 & 26 & 24 & 23 & 32 & 29 & 36 & 42 \\
\hline 689 & 25 & 25 & 24 & 25 & 24 & 23 & 29 & 29 & 35 & 41 \\
\hline 695 & 24 & 25 & 25 & 25 & 24 & 23 & 26 & 27 & 35 & 40 \\
\hline 701 & 25 & 24 & 25 & 25 & 24 & 23 & 27 & 27 & 34 & 39 \\
\hline 706 & 24 & 24 & 24 & 24 & 24 & 23 & 28 & 27 & 33 & 38 \\
\hline 712 & 24 & 24 & 24 & 24 & 24 & 23 & 28 & 28 & 33 & 37 \\
\hline 718 & 24 & 24 & 24 & 24 & 24 & 23 & 25 & 27 & 32 & 36 \\
\hline 724 & 24 & 24 & 23 & 24 & 24 & 23 & 25 & 26 & 32 & 35 \\
\hline 729 & 24 & 24 & 24 & 23 & 24 & 23 & 25 & 25 & 31 & 34 \\
\hline 735 & 24 & 24 & 24 & 24 & 24 & 23 & 25 & 25 & 31 & 34 \\
\hline 741 & 23 & 24 & 23 & 23 & 24 & 23 & 25 & 25 & 30 & 33 \\
\hline 746 & 24 & 24 & 23 & 24 & 24 & 23 & 25 & 25 & 30 & 33 \\
\hline
\end{tabular}


CLOSED DOOR TEST

THERMOCOUPLE AND DISKS AT SPRINKLER AND DETECTOR LOCATIONS - ${ }^{\circ} \mathrm{C}$

\begin{tabular}{|c|c|c|c|c|c|c|c|c|c|c|}
\hline TIME & T24 & $\mathrm{D} 25$ & T26 & $\mathrm{D} 27$ & $T 28$ & D29 & PAN A & PAN B & PAN C & PAN D \\
\hline-45 & 22 & 22 & 22 & 22 & 22 & 22 & 22 & 22 & 22 & 21 \\
\hline-38 & 22 & 22 & 22 & 22 & 22 & 22 & 22 & 22 & 22 & 22 \\
\hline-32 & 22 & 22 & 22 & 22 & 22 & 22 & 22 & 22 & 22 & 22 \\
\hline-26 & 22 & 22 & 22 & 22 & 22 & 22 & 22 & 22 & 22 & 22 \\
\hline-20 & 22 & 22 & 22 & 22 & 22 & 22 & 22 & 22 & 22 & 22 \\
\hline-15 & 22 & 22 & 22 & 22 & 22 & 22 & 22 & 22 & 22 & 22 \\
\hline-9 & 22 & 22 & 22 & 22 & 22 & 22 & 22 & 22 & 22 & 22 \\
\hline-3 & 22 & 22 & 22 & 22 & 22 & 22 & 22 & 22 & 22 & 22 \\
\hline 1 & 22 & 22 & 22 & 22 & 22 & 22 & 22 & 22 & 22 & 22 \\
\hline 7 & 22 & 22 & 22 & 22 & 22 & 22 & 23 & 22 & 22 & 22 \\
\hline 13 & 22 & 22 & 22 & 22 & 22 & 22 & 24 & 22 & 22 & 22 \\
\hline 19 & 22 & 22 & 22 & 22 & 22 & 22 & 24 & 22 & 22 & 23 \\
\hline 24 & 23 & 22 & 22 & 22 & 22 & 22 & 25 & 22 & 22 & 23 \\
\hline 30 & 24 & 22 & 23 & 22 & 22 & 22 & 25 & 22 & 22 & 25 \\
\hline 36 & 25 & 23 & 24 & 22 & 23 & 22 & 26 & 23 & 22 & 26 \\
\hline 42 & 25 & 23 & 25 & 22 & 23 & 22 & 27 & 23 & 22 & 26 \\
\hline 47 & 26 & 23 & 25 & 23 & 23 & 22 & 27 & 23 & 23 & 27 \\
\hline 53 & 27 & 24 & 26 & 23 & 24 & 23 & 28 & 24 & 23 & 27 \\
\hline 59 & 28 & 24 & 26 & 23 & 25 & 23 & 29 & 25 & 23 & 29 \\
\hline 65 & 28 & 25 & 27 & 24 & 25 & 23 & 31 & 25 & 23 & 30 \\
\hline 70 & 29 & 25 & 27 & 24 & 26 & 24 & 31 & 26 & 23 & 31 \\
\hline 76 & 30 & 25 & 28 & 24 & 27 & 24 & 31 & 27 & 24 & 31 \\
\hline 82 & 30 & 26 & 28 & 25 & 27 & 25 & 32 & 27 & 25 & 32 \\
\hline 87 & 30 & 26 & 29 & 25 & 28 & 25 & 33 & 28 & 25 & 33 \\
\hline 93 & 30 & 26 & 29 & 25 & 29 & 26 & 33 & 29 & 25 & 33 \\
\hline
\end{tabular}


CLOSED DOOR TEST

THERMOCOUPLE AND DISKS AT SPRINKLER AND DETECTOR LOCATIONS - ${ }^{\circ} \mathrm{C}$

\begin{tabular}{|c|c|c|c|c|c|c|c|c|c|c|}
\hline TIME & T24 & D25 & T26 & $\mathrm{D} 27$ & T28 & D29 & PAN A & PAN B & PAN C & PAN D \\
\hline 99 & 31 & 27 & 30 & 26 & 29 & 26 & 34 & 29 & 26 & 33 \\
\hline 105 & 31 & 27 & 30 & 26 & 29 & 26 & 34 & 29 & 26 & 34 \\
\hline 110 & 32 & 28 & 30 & 27 & 29 & 27 & 35 & 29 & 26 & 34 \\
\hline 116 & 32 & 28 & 31 & 27 & 30 & 27 & 36 & 30 & 26 & 35 \\
\hline 122 & 33 & 28 & 31 & 27 & 30 & 27 & 35 & 30 & 27 & 35 \\
\hline 128 & 34 & 29 & 32 & 28 & 30 & 28 & 36 & 30 & 27 & 36 \\
\hline 133 & 34 & 29 & 33 & 28 & 30 & 28 & 37 & 30 & 27 & 36 \\
\hline 139 & 35 & 30 & 33 & 29 & 31 & 28 & 37 & 31 & 27 & 37 \\
\hline 145 & 36 & 30 & 33 & 29 & 32 & 29 & 37 & 32 & 28 & 37 \\
\hline 151 & 36 & 31 & 34 & 29 & 32 & 29 & 39 & 32 & 28 & 38 \\
\hline 156 & 37 & 31 & 34 & 30 & 33 & 30 & 39 & 33 & 28 & 40 \\
\hline 162 & 38 & 32 & 35 & 30 & 33 & 30 & 40 & 33 & 28 & 41 \\
\hline 168 & 39 & 33 & 36 & 31 & 34 & 30 & 40 & 34 & 29 & 41 \\
\hline 173 & 40 & 33 & 36 & 31 & 34 & 31 & 42 & 34 & 30 & 41 \\
\hline 179 & 40 & 34 & 37 & 32 & 35 & 31 & 43 & 35 & 29 & 42 \\
\hline 185 & 42 & 35 & 38 & 32 & 36 & 32 & 43 & 36 & 30 & 43 \\
\hline 191 & 41 & 35 & 39 & 33 & 37 & 32 & 44 & 37 & 30 & 44 \\
\hline 196 & 41 & 36 & 39 & 33 & 36 & 33 & 45 & 36 & 30 & 44 \\
\hline 202 & 41 & 36 & 39 & 34 & 38 & 33 & 45 & 38 & 31 & 44 \\
\hline 208 & 44 & 37 & 40 & 34 & 38 & 34 & 46 & 38 & 32 & 46 \\
\hline 213 & 45 & 37 & 41 & 35 & 38 & 34 & 47 & 38 & 32 & 49 \\
\hline 219 & 44 & 38 & 42 & 35 & 38 & 35 & 48 & 38 & 33 & 48 \\
\hline 225 & 45 & 39 & 43 & 36 & 40 & 35 & 48 & 40 & 33 & 47 \\
\hline 231 & 46 & 39 & 43 & 36 & 40 & 36 & 49 & 40 & 34 & 49 \\
\hline 237 & 47 & 40 & 43 & 37 & 39 & 36 & 51 & 39 & 33 & 49 \\
\hline
\end{tabular}


THERMOCOUPLE AND DISKS AT SPRINKLER AND DETECTOR LOCATIONS - ${ }^{\circ} \mathrm{C}$

\begin{tabular}{|c|c|c|c|c|c|c|c|c|c|c|}
\hline TIME & T24 & $\mathrm{D} 25$ & $T 26$ & $\mathrm{D} 27$ & $T 28$ & D29 & PAN A & PAN B & PAN C & PAN D \\
\hline 242 & 46 & 40 & 44 & 37 & 40 & 37 & 51 & 40 & 34 & 49 \\
\hline 248 & 47 & 40 & 44 & 38 & 41 & 37 & 51 & 41 & 35 & 52 \\
\hline 254 & 48 & 41 & 44 & 38 & 42 & 38 & 53 & 42 & 35 & 52 \\
\hline 259 & 48 & 41 & 46 & 39 & 42 & 38 & 53 & 42 & 35 & 51 \\
\hline 265 & 49 & 42 & 45 & 39 & 44 & 39 & 53 & 44 & 36 & 52 \\
\hline 271 & 48 & 42 & 46 & 40 & 44 & 39 & 54 & 44 & 37 & 53 \\
\hline 277 & 50 & 43 & 47 & 40 & 45 & 40 & 55 & 45 & 37 & 53 \\
\hline 282 & 50 & 43 & 47 & 41 & 45 & 41 & 55 & 45 & 37 & 54 \\
\hline 288 & 51 & 44 & 47 & 41 & 45 & 41 & 57 & 45 & 38 & 55 \\
\hline 294 & 53 & 45 & 48 & 41 & 46 & 42 & 59 & 46 & 38 & 57 \\
\hline 299 & 52 & 45 & 50 & 42 & 46 & 42 & 59 & 46 & 38 & 55 \\
\hline 305 & 54 & 46 & 50 & 43 & 47 & 43 & 61 & 47 & 39 & 60 \\
\hline 311 & 57 & 47 & 51 & 43 & 47 & 43 & 61 & 47 & 40 & 59 \\
\hline 317 & 56 & 48 & 53 & 44 & 50 & 44 & 63 & 50 & 41 & 60 \\
\hline 322 & 58 & 49 & 53 & 45 & 49 & 44 & 66 & 49 & 40 & 61 \\
\hline 328 & 59 & 50 & 54 & 45 & 51 & 45 & 67 & 51 & 41 & 67 \\
\hline 334 & 62 & 51 & 57 & 47 & 53 & 47 & 70 & 53 & 42 & 70 \\
\hline 340 & 65 & 53 & 58 & 48 & 54 & 48 & 74 & 54 & 43 & 70 \\
\hline 345 & 68 & 54 & 61 & 49 & 58 & 49 & 75 & 58 & 45 & 72 \\
\hline 351 & 68 & 55 & 63 & 50 & 58 & 50 & 79 & 58 & 46 & 74 \\
\hline 357 & 73 & 57 & 64 & 52 & 61 & 52 & 78 & 61 & 47 & 78 \\
\hline 363 & 73 & 59 & 70 & 53 & 62 & 53 & 83 & 62 & 48 & 80 \\
\hline 368 & 77 & 61 & 71 & 55 & 63 & 55 & 84 & 63 & 51 & 79 \\
\hline 374 & 79 & 63 & 73 & 57 & 64 & 56 & 88 & 64 & 52 & 83 \\
\hline 380 & 78 & 64 & 74 & 58 & 64 & 57 & 90 & 64 & 51 & 84 \\
\hline
\end{tabular}


THERMOCOUPLE AND DISKS AT SPRINKLER AND DETECTOR LOCATIONS - ${ }^{\circ} \mathrm{C}$

\begin{tabular}{|c|c|c|c|c|c|c|c|c|c|c|}
\hline TIME & T24 & $\mathrm{D} 25$ & T26 & $\mathrm{D} 27$ & T28 & D29 & PAN A & PAN B & PAN C & PAN D \\
\hline 385 & 83 & 67 & 74 & 60 & 68 & 58 & 91 & 68 & 53 & 89 \\
\hline 391 & 84 & 68 & 75 & 61 & 68 & 60 & 91 & 68 & 54 & 88 \\
\hline 397 & 86 & 70 & 79 & 63 & 71 & 61 & 93 & 71 & 56 & 88 \\
\hline 403 & 88 & 72 & 79 & 64 & 73 & 63 & 96 & 73 & 57 & 91 \\
\hline 408 & 91 & 74 & 83 & 66 & 73 & 64 & 97 & 73 & 58 & 96 \\
\hline 414 & 93 & 76 & 87 & 67 & 76 & 66 & 101 & 76 & 57 & 96 \\
\hline 420 & 95 & 78 & 82 & 69 & 78 & 67 & 102 & 78 & 60 & 97 \\
\hline 426 & 95 & 79 & 85 & 70 & 78 & 69 & 103 & 78 & 61 & 98 \\
\hline 431 & 95 & 81 & 87 & 71 & 79 & 70 & 104 & 79 & 62 & 101 \\
\hline 437 & 95 & 82 & 89 & 73 & 79 & 71 & 107 & 79 & 62 & 98 \\
\hline 443 & 96 & 83 & 88 & 74 & 82 & 72 & 104 & 82 & $\approx 2$ & 97 \\
\hline 448 & 95 & 84 & 89 & 75 & 81 & 73 & 103 & 81 & 65 & 100 \\
\hline 454 & 98 & 85 & 88 & 75 & 81 & 74 & 103 & 81 & 63 & 97 \\
\hline 460 & 97 & 86 & 89 & 76 & 80 & 74 & 101 & 80 & 65 & 99 \\
\hline 466 & 94 & 86 & 87 & 76 & 81 & 74 & 103 & 81 & 65 & 97 \\
\hline 471 & 93 & 86 & 86 & 76 & 80 & 75 & 104 & 80 & 63 & 99 \\
\hline 477 & 97 & 86 & 87 & 77 & 80 & 75 & 104 & 80 & 63 & 101 \\
\hline 483 & 98 & 87 & 86 & 77 & 81 & 76 & 108 & 81 & 61 & 101 \\
\hline 489 & 99 & 87 & 90 & 78 & 83 & 77 & 108 & 83 & 64 & 103 \\
\hline 494 & 100 & 88 & 93 & 78 & 85 & 77 & 113 & 85 & 65 & 102 \\
\hline 500 & 101 & 89 & 94 & 79 & 84 & 78 & 113 & 84 & 64 & 100 \\
\hline 506 & 56 & 80 & 91 & 80 & 84 & 79 & 113 & 84 & 67 & 104 \\
\hline 512 & 68 & 57 & 97 & 81 & 85 & 79 & 116 & 85 & 65 & 112 \\
\hline 517 & 98 & 53 & 96 & 82 & 89 & 81 & 117 & 89 & 67 & 111 \\
\hline 523 & 106 & 53 & 100 & 83 & 91 & 82 & 118 & 91 & 69 & 111 \\
\hline
\end{tabular}


THERMOCOUPLE AND DISKS AT SPRINKLER AND DETECTOR LOCATIONS - ${ }^{\circ} \mathrm{C}$

\begin{tabular}{|c|c|c|c|c|c|c|c|c|c|c|}
\hline TIME & $T 24$ & D25 & T26 & $\mathrm{D} 27$ & T28 & D29 & PAN A & PAN B & PAN C & PAN D \\
\hline 529 & 107 & 56 & 100 & 84 & 89 & 82 & 124 & 89 & 70 & 113 \\
\hline 535 & 107 & 64 & 103 & 86 & 92 & 83 & 127 & 92 & 71 & 115 \\
\hline 540 & 107 & 71 & 103 & 87 & 95 & 84 & 125 & 95 & 70 & 118 \\
\hline 546 & 111 & $\pi$ & 99 & 87 & 95 & 86 & 127 & 95 & 72 & 118 \\
\hline 552 & 114 & 82 & 108 & 88 & 96 & 87 & 129 & 96 & 75 & 120 \\
\hline 557 & 114 & 87 & 106 & 89 & 98 & 88 & 128 & 98 & 75 & 124 \\
\hline 563 & 118 & 90 & 109 & 91 & 98 & 89 & 131 & 98 & $\pi$ & 122 \\
\hline 569 & 123 & 95 & 110 & 92 & 101 & 90 & 134 & 101 & 77 & 124 \\
\hline 575 & 122 & 97 & 108 & 93 & 104 & 92 & 128 & 104 & 78 & 125 \\
\hline 580 & 38 & 44 & 38 & 69 & 22 & 22 & 34 & 22 & 79 & 35 \\
\hline 586 & 42 & 42 & 38 & 46 & 21 & 22 & 36 & 21 & 73 & 42 \\
\hline 592 & 38 & 39 & 33 & 38 & 21 & 21 & 33 & 21 & 61 & 42 \\
\hline 598 & 37 & 36 & 31 & 34 & 20 & 21 & 31 & 20 & 56 & 42 \\
\hline 603 & 31 & 33 & 30 & 32 & 21 & 21 & 30 & 21 & 52 & 40 \\
\hline 609 & 33 & 32 & 29 & 31 & 20 & 21 & 29 & 20 & 48 & 41 \\
\hline 615 & 31 & 32 & 28 & 30 & 20 & 20 & 29 & 20 & 45 & 47 \\
\hline 620 & 29 & 30 & 28 & 29 & 20 & 20 & 28 & 20 & 43 & 44 \\
\hline 626 & 29 & 29 & 28 & 28 & 21 & 21 & 27 & 21 & 41 & 46 \\
\hline 632 & 27 & 28 & 27 & 28 & 20 & 21 & 27 & 20 & 40 & 42 \\
\hline 638 & 27 & 27 & 26 & 27 & 21 & 21 & 26 & 21 & 39 & 44 \\
\hline 643 & 27 & 27 & 27 & 27 & 20 & 21 & 26 & 20 & 38 & 42 \\
\hline 649 & 29 & 27 & 26 & 26 & 20 & 20 & 26 & 20 & 35 & 42 \\
\hline 655 & 26 & 27 & 26 & 26 & 20 & 20 & 25 & 20 & 36 & 39 \\
\hline 661 & 26 & 26 & 26 & 26 & 21 & 21 & 25 & 21 & 36 & 34 \\
\hline 666 & 27 & 26 & 25 & 25 & 20 & 20 & 25 & 20 & 36 & 33 \\
\hline
\end{tabular}


CLOSED DOOR TEST

THERMOCOUPLE AND DISKS AT SPRINKLER AND DETECTOR LOCATIONS - ${ }^{\circ} \mathrm{C}$

\begin{tabular}{|c|c|c|c|c|c|c|c|c|c|c|}
\hline TIME & T24 & D25 & T26 & D27 & T28 & D29 & PAN A & PAN B & PAN C & PAN D \\
\hline 672 & 25 & 25 & 25 & 25 & 20 & $20^{\circ}$ & 25 & 20 & 36 & 32 \\
\hline 678 & 25 & 25 & 24 & 25 & 20 & 20 & 25 & 20 & 35 & 34 \\
\hline 683 & 24 & 24 & 25 & 25 & 20 & 20 & 24 & 20 & 35 & 32 \\
\hline 689 & 25 & 24 & 24 & 24 & 20 & 20 & 24 & 20 & 34 & 29 \\
\hline 695 & 24 & 24 & 24 & 24 & 20 & 20 & 23 & 20 & 34 & 26 \\
\hline 701 & 24 & 24 & 24 & 24 & 20 & 20 & 24 & 20 & 33 & 27 \\
\hline 706 & 24 & 24 & 24 & 24 & 20 & 20 & 23 & 20 & 33 & 28 \\
\hline 712 & 24 & 24 & 24 & 24 & 20 & 20 & 24 & 20 & 33 & 28 \\
\hline 718 & 24 & 24 & 24 & 24 & 20 & 20 & 23 & 20 & 33 & 25 \\
\hline 724 & 24 & 24 & 23 & 23 & 20 & 20 & 23 & 20 & 32 & 25 \\
\hline 729 & 23 & 23 & 24 & 23 & 20 & 21 & 23 & 20 & 31 & 25 \\
\hline 735 & 24 & 23 & 24 & 23 & 29 & 21 & 24 & 21 & 31 & 25 \\
\hline 749 & 24 & 23 & 23 & 23 & 21 & 21 & 23 & 21 & 32 & 25 \\
\hline 746 & 24 & 23 & 23 & 23 & 29 & 21 & 23 & 21 & 32 & 25 \\
\hline
\end{tabular}


CLOSED DOOR PAIVACY CURTAIN TEST

THERMOCOUPLES AND DISKS AT SPRINKLER AND DETECTOR LOCATIONS - ${ }^{\circ} \mathrm{C}$

\begin{tabular}{|c|c|c|c|c|c|c|c|c|c|c|}
\hline TIME & T14 & D15 & $T 16$ & D17 & T18 & D19 & $T 20$ & $\mathrm{D} 21$ & T22 & $\mathrm{D} 23$ \\
\hline-54 & 24 & 24 & 24 & 24 & 25 & 25 & 24 & 24 & 24 & 24 \\
\hline-48 & 24 & 24 & 25 & 24 & 25 & 25 & 24 & 24 & 24 & 24 \\
\hline-42 & 24 & 24 & 25 & 24 & 25 & 25 & 24 & 24 & 24 & 24 \\
\hline-37 & 24 & 24 & 25 & 24 & 25 & 25 & 24 & 24 & 24 & 24 \\
\hline .31 & 24 & 24 & 25 & 24 & 25 & 25 & 24 & 24 & 24 & 24 \\
\hline-25 & 24 & 24 & 25 & 24 & 25 & 25 & 24 & 24 & 24 & 24 \\
\hline-19 & 24 & 24 & 25 & 24 & 25 & 25 & 24 & 24 & 24 & 24 \\
\hline-14 & 24 & 24 & 25 & 24 & 25 & 25 & 24 & 24 & 24 & 24 \\
\hline-8 & 24 & 24 & 25 & 24 & 25 & 25 & 24 & 24 & 24 & 24 \\
\hline-2 & 25 & 24 & 25 & 24 & 25 & 25 & 24 & 24 & 24 & 24 \\
\hline 3 & 25 & 24 & 25 & 24 & 25 & 25 & 24 & 24 & 24 & 24 \\
\hline 8 & 25 & 24 & 27 & 25 & 25 & 25 & 24 & 24 & 25 & 25 \\
\hline 14 & 25 & 24 & 30 & 26 & 25 & 25 & 25 & 24 & 27 & 25 \\
\hline 20 & 26 & 25 & 30 & 27 & 25 & 25 & 27 & 25 & 26 & 25 \\
\hline 26 & 27 & 25 & 31 & 27 & 26 & 25 & 27 & 25 & 27 & 25 \\
\hline 31 & 28 & 25 & 31 & 28 & 28 & 25 & 28 & 25 & 28 & 26 \\
\hline 37 & 28 & 26 & 32 & 29 & 28 & 26 & 29 & 26 & 28 & 26 \\
\hline 43 & 28 & 26 & 34 & 30 & 29 & 26 & 29 & 26 & 28 & 27 \\
\hline 49 & 29 & 26 & 34 & 31 & 29 & 27 & 30 & 27 & 28 & 27 \\
\hline 54 & 30 & 27 & 36 & 32 & 31 & 27 & 30 & 27 & 29 & 27 \\
\hline 60 & 30 & 27 & 37 & 32 & 31 & 28 & 31 & 28 & 30 & 28 \\
\hline 66 & 31 & 28 & 37 & 33 & 32 & 28 & 31 & 28 & 30 & 28 \\
\hline 72 & 32 & 28 & 38 & 34 & 32 & 28 & 32 & 28 & 31 & 29 \\
\hline 77 & 33 & 29 & 39 & 35 & 32 & 29 & 32 & 29 & 32 & 29 \\
\hline 83 & 34 & 30 & 39 & 36 & 32 & 29 & 33 & 29 & 31 & 29 \\
\hline
\end{tabular}


CLOSED DOOR PRIVACY CURTAIN TEST

\begin{tabular}{|c|c|c|c|c|c|c|c|c|c|c|}
\hline TIME & $T 14$ & D15 & T16 & 017 & T18 & D19 & T20 & D21 & T22 & $\mathrm{D} 23$ \\
\hline 89 & 34 & 30 & 38 & 36 & 33 & 30 & 34 & 30 & 32 & 30 \\
\hline 95 & 34 & 31 & 40 & 37 & 34 & 30 & 34 & 30 & 33 & 31 \\
\hline 100 & 35 & 31 & 41 & 38 & 34 & 30 & 35 & 31 & 33 & 31 \\
\hline 106 & 35 & 32 & 42 & 39 & 35 & 31 & 36 & 31 & 34 & 32 \\
\hline 112 & 36 & 32 & 44 & 40 & 36 & 32 & 35 & 32 & 35 & 32 \\
\hline 118 & 38 & 33 & 45 & 41 & 37 & 32 & 36 & 32 & 35 & 33 \\
\hline 123 & 38 & 34 & 45 & 42 & 37 & 33 & 37 & 33 & 36 & 33 \\
\hline 129 & 38 & 34 & 46 & 42 & 37 & 33 & 38 & 33 & 37 & 34 \\
\hline 135 & 39 & 35 & 47 & 43 & 37 & 33 & 38 & 34 & 37 & 35 \\
\hline 141 & 39 & 36 & 48 & 44 & 38 & 34 & 38 & 34 & 38 & 35 \\
\hline 146 & 40 & 36 & 49 & 45 & 39 & 35 & 39 & 35 & 38 & 36 \\
\hline 152 & 41 & 37 & 50 & 46 & 39 & 35 & 40 & 35 & 40 & 37 \\
\hline 158 & 41 & 37 & 51 & 47 & 41 & 36 & 41 & 36 & 42 & 38 \\
\hline 164 & 42 & 38 & 51 & 47 & 42 & 36 & 42 & 37 & 41 & 39 \\
\hline 169 & 43 & 39 & 51 & 48 & 42 & 37 & 42 & 37 & 40 & 39 \\
\hline 175 & 43 & 39 & 53 & 49 & 44 & 38 & 44 & 38 & 43 & 40 \\
\hline 181 & 44 & 40 & 54 & 50 & 44 & 38 & 45 & 39 & 45 & 41 \\
\hline 187 & 45 & 40 & 55 & 51 & 45 & 39 & 46 & 40 & 44 & 42 \\
\hline 192 & 45 & 41 & 56 & 51 & 45 & 40 & 46 & 40 & 44 & 42 \\
\hline 198 & 46 & 42 & 56 & 52 & 47 & 41 & 47 & 41 & 43 & 43 \\
\hline 204 & 47 & 42 & 58 & 53 & 46 & 41 & 47 & 42 & 46 & 43 \\
\hline 210 & 47 & 43 & 59 & 54 & 47 & 42 & 48 & 42 & 45 & 44 \\
\hline 215 & 48 & 44 & 59 & 55 & 47 & 42 & 48 & 43 & 47 & 45 \\
\hline 221 & 49 & 44 & 60 & 56 & 48 & 43 & 49 & 43 & 47 & 45 \\
\hline 227 & 50 & 45 & 60 & 56 & 47 & 43 & 49 & 44 & 52 & 47 \\
\hline
\end{tabular}


CLOSED DOOR PRIVACY CURTAIN TEST

THERMOCOUPLES AND DISKS AT SPRINKLER AND DETECTOR LOCATIONS - ${ }^{\circ} \mathrm{C}$

\begin{tabular}{|c|c|c|c|c|c|c|c|c|c|c|}
\hline TIME & $T_{14}$ & D15 & $\mathrm{T} 16$ & D17 & T18 & D19 & $T 20$ & D21 & T22 & $\mathrm{D} 23$ \\
\hline 233 & 49 & 45 & 61 & 57 & 48 & 44 & 50 & 45 & 50 & 47 \\
\hline 238 & 49 & 46 & 62 & 58 & 50 & 44 & 51 & 45 & 51 & 48 \\
\hline 244 & 51 & 47 & 66 & 60 & 50 & 45 & 51 & 46 & 50 & 49 \\
\hline 250 & 52 & 47 & 65 & 60 & 51 & 45 & 51 & 47 & 48 & 49 \\
\hline 256 & 53 & 48 & 65 & 61 & 51 & 46 & 52 & 47 & 50 & 50 \\
\hline 261 & 53 & 48 & 67 & 62 & 51 & 46 & 53 & 48 & 51 & 50 \\
\hline 267 & 53 & 49 & 67 & 63 & 52 & 47 & 53 & 48 & 52 & 51 \\
\hline 273 & 54 & 50 & 67 & 63 & 54 & 48 & 54 & 49 & 53 & 51 \\
\hline 279 & 55 & 50 & 66 & 64 & 54 & 48 & 54 & 49 & 54 & 52 \\
\hline 284 & 56 & 51 & 69 & 64 & 54 & 49 & 55 & 50 & 55 & 53 \\
\hline 290 & 55 & 51 & 69 & 65 & 54 & 49 & 56 & 51 & 56 & 53 \\
\hline 296 & 56 & 52 & 70 & 66 & 54 & 49 & 56 & 51 & 56 & 54 \\
\hline 301 & 59 & 53 & 70 & 66 & 55 & 50 & 57 & 52 & 56 & 54 \\
\hline 307 & 59 & 54 & 71 & 67 & 55 & 50 & 56 & 52 & 57 & 55 \\
\hline 313 & 59 & 54 & 74 & 69 & 56 & 51 & 57 & 53 & 60 & 56 \\
\hline 319 & 61 & 55 & 75 & 69 & 57 & 51 & 59 & 53 & 62 & 57 \\
\hline 324 & 61 & 56 & 75 & 70 & 59 & 52 & 60 & 54 & 63 & 58 \\
\hline 330 & 62 & 57 & 75 & 71 & 59 & 53 & 62 & 55 & 62 & 59 \\
\hline 336 & 64 & 57 & 79 & 73 & 60 & 54 & 63 & 56 & 66 & 61 \\
\hline 342 & 63 & 58 & 78 & 73 & 60 & 54 & 61 & 56 & 66 & 62 \\
\hline 348 & 65 & 59 & 82 & 75 & 61 & 55 & 62 & 57 & 67 & 63 \\
\hline 353 & 65 & 60 & 79 & 75 & 63 & 55 & 63 & 58 & 64 & 63 \\
\hline 359 & 65 & 60 & 79 & 76 & 62 & 56 & 66 & 59 & 67 & 64 \\
\hline 365 & 65 & 61 & 83 & 77 & 63 & 57 & 65 & 59 & 66 & 65 \\
\hline 371 & 66 & 61 & 79 & 77 & 64 & 57 & 66 & 60 & 67 & 66 \\
\hline
\end{tabular}


CLOSED DOOR PRIVACY CURTAIN TEST

THERMOCOUPLES AND DISKS AT SPRINKLER AND DETECTOR LOCATIONS . ${ }^{\circ} \mathrm{C}$

\begin{tabular}{|c|c|c|c|c|c|c|c|c|c|c|}
\hline TIME & $\mathrm{T} 14$ & D15 & T16 & D17 & T18 & D19 & T2O & D21 & T22 & D23 \\
\hline 376 & 67 & 62 & 83 & 78 & 64 & 58 & .65 & 60 & 65 & 66 \\
\hline 382 & 68 & 63 & 85 & 80 & 65 & 58 & 67 & 61 & 69 & 67 \\
\hline 388 & 70 & 64 & 86 & 80 & 64 & 59 & 68 & 62 & 72 & 68 \\
\hline 394 & 71 & 65 & 86 & 81 & 66 & 59 & 69 & 62 & 70 & 68 \\
\hline 399 & 71 & 65 & 84 & 82 & 67 & 60 & 70 & 63 & 71 & 69 \\
\hline 405 & 72 & 66 & 90 & 84 & 67 & 60 & 70 & 64 & 70 & 70 \\
\hline 411 & 71 & 66 & 89 & 84 & 68 & 61 & 70 & 64 & 70 & 70 \\
\hline 496 & 73 & 67 & 91 & 85 & 68 & 62 & 79 & 65 & 75 & 71 \\
\hline 422 & 73 & 68 & 89 & 84 & 69 & 62 & 71 & 66 & 76 & 72 \\
\hline 428 & 77 & 69 & 94 & 86 & 71 & 63 & 72 & 66 & 78 & 74 \\
\hline 434 & 77 & 70 & 94 & 88 & 71 & 64 & 73 & 67 & 80 & 75 \\
\hline 439 & 77 & 71 & 98 & 90 & 74 & 65 & 75 & 67 & 79 & 76 \\
\hline 445 & 78 & 71 & 99 & 92 & 75 & 66 & 74 & 68 & 82 & 77 \\
\hline 451 & 79 & 72 & 98 & 92 & 73 & 66 & 78 & 69 & 79 & 78 \\
\hline 457 & 80 & 73 & 97 & 93 & 76 & 67 & 77 & 70 & 81 & 79 \\
\hline 462 & 79 & 74 & 100 & 94 & 76 & 68 & 77 & 71 & 83 & 80 \\
\hline 468 & 80 & 74 & 97 & 94 & 75 & 68 & 78 & 72 & 80 & 80 \\
\hline 474 & 80 & 75 & 96 & 94 & 74 & 69 & 77 & 72 & 80 & 80 \\
\hline 480 & 79 & 75 & 96 & 94 & 75 & 69 & 77 & 72 & 81 & 81 \\
\hline 485 & 78 & 75 & 97 & 94 & 76 & 69 & 75 & 72 & 81 & 81 \\
\hline 491 & 78 & 75 & 93 & 93 & 76 & 69 & 77 & 72 & 79 & 81 \\
\hline 497 & 79 & 75 & 93 & 92 & 75 & 70 & 76 & 73 & 81 & 81 \\
\hline 503 & 79 & 75 & 92 & 92 & 75 & 70 & 76 & 73 & 82 & 81 \\
\hline 508 & 79 & 75 & 94 & 92 & 74 & 70 & 76 & 73 & 86 & 82 \\
\hline 594 & 78 & 75 & 94 & 92 & 74 & 70 & 77 & 73 & 80 & 82 \\
\hline
\end{tabular}


THERMOCOUPLES AND DISKS AT SPRINKLER AND DETECTOR LOCATIONS - ${ }^{\circ} \mathrm{C}$

\begin{tabular}{|c|c|c|c|c|c|c|c|c|c|c|}
\hline TIME & $T 14$ & D15 & T16 & D17 & T18 & D19 & T20 & $\mathrm{D} 21$ & T22 & $\mathrm{D} 23$ \\
\hline 520 & 80 & 76 & 95 & 92 & 76 & 70 & 77 & 73 & 81 & 82 \\
\hline 526 & 77 & 75 & 95 & 92 & 76 & 70 & 78 & 74 & 85 & 82 \\
\hline 531 & 79 & 75 & 99 & 94 & 76 & 70 & 77 & 74 & 83 & 83 \\
\hline 537 & 80 & 76 & 98 & 94 & 74 & 70 & 81 & 74 & 84 & 84 \\
\hline 543 & 82 & 76 & 99 & 95 & 78 & 71 & 80 & 75 & 86 & 84 \\
\hline 549 & 82 & 77 & 99 & 95 & 76 & 71 & 80 & 75 & 86 & 84 \\
\hline 554 & 84 & 78 & 101 & 97 & 80 & 72 & 81 & 76 & 87 & 85 \\
\hline 560 & 83 & 78 & 104 & 98 & 82 & 72 & 83 & 76 & 89 & 86 \\
\hline 566 & 84 & 79 & 102 & 98 & 82 & 73 & 81 & 77 & 88 & 87 \\
\hline 572 & 41 & 63 & 104 & 99 & 79 & 74 & 81 & 77 & 91 & 87 \\
\hline 577 & 44 & 55 & 103 & 99 & 82 & 74 & 84 & 78 & 93 & 88 \\
\hline 583 & 64 & 54 & 107 & 100 & 83 & 75 & 85 & 79 & 92 & 89 \\
\hline 589 & 79 & 59 & 109 & 101 & 81 & 75 & 85 & 79 & 93 & 90 \\
\hline 595 & 80 & 64 & 106 & 102 & 86 & 76 & 86 & 80 & 96 & 92 \\
\hline 600 & 81 & 68 & 110 & 104 & 82 & 76 & 87 & 81 & 97 & 93 \\
\hline 606 & 83 & 71 & 109 & 104 & 86 & 77 & 88 & 81 & 94 & 93 \\
\hline 612 & 84 & 74 & 109 & 105 & 86 & 78 & 87 & 82 & 96 & 94 \\
\hline 618 & 87 & 77 & 113 & 106 & 87 & 78 & 86 & 82 & 99 & 95 \\
\hline 623 & 87 & 80 & 913 & 107 & 88 & 79 & 88 & 83 & 100 & 95 \\
\hline 629 & 86 & 81 & 113 & 108 & 89 & 80 & 89 & 83 & 103 & 97 \\
\hline 635 & 89 & 83 & 117 & 110 & 87 & 80 & 90 & 84 & 105 & 98 \\
\hline 641 & 92 & 85 & 117 & 111 & 91 & 81 & 94 & 85 & 106 & 100 \\
\hline 646 & 97 & 87 & 118 & 113 & 92 & 82 & 94 & 86 & 104 & 101 \\
\hline 652 & 97 & 88 & 119 & 113 & 95 & 84 & 95 & 87 & 105 & 102 \\
\hline 658 & 98 & 90 & 122 & 115 & 94 & 84 & 98 & 89 & 109 & 103 \\
\hline
\end{tabular}




\begin{tabular}{|c|c|c|c|c|c|c|c|c|c|c|}
\hline \multicolumn{11}{|c|}{ THERMOCOUPLES AND DISKS AT SPRINKLER AND DETECTOR LOCATIONS - ${ }^{\circ} \mathrm{C}$} \\
\hline TIME & T14 & D15 & T16 & D17 & T18 & D19 & T20 & $\mathrm{D} 21$ & T22 & $\mathrm{D} 23$ \\
\hline 664 & 101 & 91 & 123 & 117 & 94 & 85 & 98 & 89 & 105 & 104 \\
\hline 669 & 103 & 93 & 128 & 119 & 98 & 86 & 101 & 90 & 113 & 106 \\
\hline 675 & 103 & 94 & 125 & 120 & 99 & 87 & 104 & 92 & 118 & 109 \\
\hline 681 & 107 & 96 & 131 & 122 & 98 & 88 & 104 & 93 & 121 & 111 \\
\hline 687 & 106 & 97 & 131 & 123 & 102 & 89 & 105 & 95 & 116 & 112 \\
\hline 692 & 111 & 100 & 129 & 124 & 101 & 90 & 106 & 96 & 121 & 114 \\
\hline 698 & 111 & 101 & 137 & 128 & 104 & 92 & 109 & 98 & 118 & 114 \\
\hline 704 & 114 & 103 & 141 & 131 & 107 & 93 & 111 & 99 & 123 & 116 \\
\hline 710 & 113 & 104 & 141 & 133 & 113 & 95 & 112 & 101 & 120 & 117 \\
\hline 716 & 114 & 105 & 143 & 135 & 911 & 97 & 114 & 102 & 127 & 119 \\
\hline 721 & 116 & 107 & 144 & 136 & 191 & 98 & 114 & 104 & 128 & 120 \\
\hline 727 & 117 & 108 & 145 & 138 & 111 & 99 & 113 & 105 & 131 & 123 \\
\hline 733 & 119 & 109 & 148 & 140 & 110 & 100 & 117 & 107 & 131 & 124 \\
\hline 739 & 121 & 119 & 148 & 149 & 113 & 101 & 118 & 108 & 129 & 125 \\
\hline 745 & 124 & 113 & 153 & 144 & 115 & 102 & 118 & 109 & 135 & 127 \\
\hline 751 & 126 & 114 & 153 & 145 & 120 & 104 & 120 & 110 & 137 & 130 \\
\hline 757 & 127 & 116 & 155 & 147 & 117 & 105 & 119 & 111 & 135 & 131 \\
\hline 763 & 124 & 114 & 51 & 198 & 42 & 49 & 61 & 63 & 123 & 130 \\
\hline 769 & 62 & 103 & 42 & 46 & 37 & 36 & 46 & 46 & 118 & 126 \\
\hline 775 & 46 & 92 & 39 & 41 & 35 & 34 & 38 & 42 & 101 & 116 \\
\hline 780 & 41 & 82 & 39 & 40 & 34 & 33 & 44 & 41 & 101 & 112 \\
\hline 786 & 39 & 73 & 39 & 37 & 34 & 33 & 43 & 41 & 90 & 106 \\
\hline 792 & 39 & 68 & 35 & 36 & 33 & 33 & 36 & 38 & 87 & 99 \\
\hline 798 & 39 & 63 & 37 & 36 & 34 & 33 & 41 & 39 & 84 & 82 \\
\hline 804 & 38 & 59 & 37 & 35 & 33 & 32 & 39 & 39 & 86 & 77 \\
\hline
\end{tabular}


THERMOCOUPLES AND DISKS AT SPRINKLER AND DETECTOR LOCATIONS - ${ }^{\circ} \mathrm{C}$

\begin{tabular}{|c|c|c|c|c|c|c|c|c|c|c|}
\hline TIME & $T 14$ & D15 & T16 & D17 & $T 18$ & D19 & $T 20$ & D21 & T22 & $\mathrm{D} 23$ \\
\hline 810 & 37 & 55 & 36 & 35 & 33 & 32 & 36 & 38 & 83 & 78 \\
\hline 816 & 37 & 52 & 35 & 34 & 32 & 32 & 38 & 38 & 79 & 71 \\
\hline 822 & 37 & 49 & 36 & 34 & 33 & 32 & 39 & 38 & 76 & 67 \\
\hline 827 & 36 & 47 & 36 & 35 & 32 & 31 & 38 & 37 & 80 & 70 \\
\hline 833 & 36 & 45 & 34 & 34 & 32 & 31 & 37 & 37 & 79 & 72 \\
\hline 839 & 36 & 43 & 34 & 33 & 32 & 31 & 38 & 37 & 78 & 73 \\
\hline 845 & 36 & 42 & 34 & 33 & 32 & 31 & 40 & 37 & 73 & 72 \\
\hline 851 & 35 & 41 & 34 & 33 & 32 & 31 & 38 & 37 & 81 & 74 \\
\hline 857 & 35 & 40 & 35 & 33 & 32 & 31 & 37 & 36 & 71 & 73 \\
\hline 862 & 35 & 39 & 34 & 33 & 32 & 31 & 36 & 36 & 78 & 74 \\
\hline 868 & 35 & 38 & 33 & 33 & 31 & 31 & 35 & 35 & 80 & 75 \\
\hline 874 & 35 & 38 & 35 & 33 & 32 & 31 & 40 & 36 & 73 & 74 \\
\hline 880 & 35 & 37 & 34 & 33 & 32 & 31 & 33 & 35 & 76 & 74 \\
\hline 886 & 35 & 36 & 34 & 33 & 32 & 31 & 40 & 36 & 76 & 74 \\
\hline 892 & 35 & 36 & 35 & 33 & 32 & 31 & 37 & 36 & 75 & 74 \\
\hline 898 & 35 & 35 & 34 & 33 & 32 & 31 & 35 & 35 & 64 & 73 \\
\hline 904 & 35 & 35 & 34 & 33 & 31 & 31 & 36 & 35 & 73 & 73 \\
\hline 909 & 35 & 35 & 34 & 33 & 31 & 31 & 37 & 36 & 72 & 72 \\
\hline 915 & 34 & 35 & 33 & 32 & 31 & 31 & 36 & 36 & 73 & 72 \\
\hline 921 & 34 & 35 & 34 & 32 & 31 & 31 & 36 & 36 & 74 & 72 \\
\hline 927 & 33 & 34 & 33 & 32 & 31 & 30 & 34 & 35 & 71 & 71 \\
\hline 933 & 34 & 34 & 34 & 32 & 31 & 31 & 35 & 34 & 74 & 71 \\
\hline 939 & 33 & 34 & 32 & 32 & 31 & 30 & 33 & 33 & 70 & 70 \\
\hline 945 & 33 & 34 & 34 & 32 & 31 & 30 & 33 & 34 & 68 & 72 \\
\hline 951 & 33 & 33 & 33 & 32 & 31 & 31 & 37 & 34 & 75 & 72 \\
\hline
\end{tabular}


CLOSED DOOR PRIVACY CURTAIN TEST

\begin{tabular}{|c|c|c|c|c|c|c|c|c|c|c|}
\hline \multicolumn{11}{|c|}{ THERMOCOUPLES AND DISKS AT SPRINKLER AND DETECTOR LOCATIONS - ${ }^{\circ} \mathrm{C}$} \\
\hline TIME & T14 & D15 & T16 & 017 & T18 & D19 & T20 & D21 & T22 & D23 \\
\hline 957 & 33 & 33 & 33 & 32 & 31 & $31 \cdot$ & 34 & 34 & 72 & 72 \\
\hline 962 & 33 & 33 & 34 & 32 & 32 & 31 & 36 & 34 & 72 & 71 \\
\hline 968 & 34 & 33 & 35 & 33 & 32 & 32 & 36 & 35 & 71 & 71 \\
\hline 974 & 34 & 33 & 34 & 33 & 33 & 33 & 35 & 35 & 68 & 70 \\
\hline 980 & 34 & 34 & 33 & 33 & 33 & 33 & 33 & 34 & 67 & 69 \\
\hline 986 & 34 & 34 & 33 & 33 & 33 & 33 & 34 & 34 & 67 & 68 \\
\hline 992 & 34 & 34 & 33 & 33 & 33 & 33 & 33 & 33 & 61 & 65 \\
\hline 997 & 34 & 34 & 34 & 33 & 34 & 33 & 34 & 34 & 55 & 62 \\
\hline 1003.0 & 34 & 34 & 34 & 33 & 34 & 33 & 34 & 34 & 54 & 60 \\
\hline 1009.0 & 33 & 33 & 34 & 34 & 34 & 34 & 34 & 34 & 52 & 58 \\
\hline 1015.0 & 33 & 33 & 34 & 34 & 34 & 34 & 34 & 34 & 49 & 56 \\
\hline 1021.0 & 34 & 33 & 34 & 34 & 34 & 34 & 34 & 34 & 48 & 54 \\
\hline 1027.0 & 34 & 34 & 34 & 34 & 34 & 34 & 34 & 34 & 47 & 53 \\
\hline 1032.0 & 33 & 34 & 34 & 34 & 34 & 34 & 34 & 34 & 47 & 52 \\
\hline 1038.0 & 34 & 34 & 34 & 34 & 34 & 34 & 34 & 34 & 47 & 51 \\
\hline 1044.0 & 34 & 34 & 34 & 34 & 34 & 34 & 33 & 34 & 48 & 50 \\
\hline 1050.0 & 34 & 34 & 34 & 34 & 34 & 34 & 34 & 34 & 47 & 49 \\
\hline 1056.0 & 34 & 34 & 34 & 34 & 34 & 34 & 34 & 34 & 46 & 48 \\
\hline 1062.0 & 34 & 34 & 34 & 34 & 34 & 34 & 34 & 34 & 47 & 48 \\
\hline 1067.0 & 34 & 34 & 34 & 34 & 34 & 34 & 34 & 34 & 45 & 47 \\
\hline 1073.0 & 34 & 34 & 34 & 34 & 34 & 34 & 34 & 34 & 47 & 47 \\
\hline 1079.0 & 34 & 34 & 34 & 34 & 34 & 34 & 34 & 34 & 43 & 46 \\
\hline 1085.0 & 34 & 34 & 34 & 34 & 34 & 34 & 34 & 34 & 43 & 45 \\
\hline 1098.0 & 34 & 34 & 34 & 34 & 34 & 34 & 33 & 34 & 43 & 44 \\
\hline 1097.0 & 34 & 34 & 34 & 34 & 34 & 34 & 33 & 33 & 42 & 43 \\
\hline
\end{tabular}


THERMOCOUPLES AND DISKS AT SPRINKLER AND DETECTOR LOCATIONS - ${ }^{\circ} \mathrm{C}$

\begin{tabular}{|c|c|c|c|c|c|c|c|c|c|c|}
\hline TME & $T_{14}$ & D15 & T16 & D17 & $T 18$ & D19 & $T 20$ & D21 & $\mathrm{T} 22$ & D23 \\
\hline 1102.0 & 34 & 34 & 34 & 34 & 34 & 34 & 33 & 33 & 41 & 42 \\
\hline 1108.0 & 34 & 34 & 34 & 34 & 34 & 34 & 34 & 34 & 40 & 42 \\
\hline 1114.0 & 34 & 34 & 34 & 34 & 34 & 34 & 33 & 34 & 41 & 41 \\
\hline 1120.0 & 34 & 34 & 34 & 34 & 34 & 34 & 33 & 33 & 40 & 41 \\
\hline 1126.0 & 33 & 34 & 34 & 34 & 34 & 34 & 33 & 33 & 41 & 40 \\
\hline 1132.0 & 33 & 33 & 34 & 34 & 34 & 34 & 33 & 33 & 40 & 40 \\
\hline 1137.0 & 33 & 33 & 34 & 34 & 34 & 34 & 33 & 33 & 41 & 40 \\
\hline 1143.0 & 34 & 34 & 34 & 34 & 34 & 33 & 33 & 33 & 39 & 39 \\
\hline 1149.0 & 33 & 33 & 34 & 34 & 33 & 34 & 33 & 33 & 39 & 39 \\
\hline 1155.0 & 33 & 33 & 34 & 34 & 34 & 34 & 33 & 33 & 39 & 39 \\
\hline 1161.0 & 34 & 33 & 34 & 34 & 34 & 34 & 33 & 33 & 39 & 39 \\
\hline 1167.0 & 33 & 33 & 33 & 34 & 34 & 34 & 33 & 33 & 38 & 38 \\
\hline 1172.0 & 34 & 33 & 34 & 34 & 34 & 34 & 33 & 33 & 38 & 38 \\
\hline 1178.0 & 34 & 33 & 33 & 34 & 33 & 33 & 33 & 33 & 37 & 38 \\
\hline 1184.0 & 33 & 33 & 34 & 34 & 34 & 33 & 33 & 33 & 38 & 38 \\
\hline 1190.0 & 33 & 33 & 33 & 34 & 34 & 33 & 33 & 33 & 37 & 37 \\
\hline 1196.0 & 33 & 33 & 33 & 33 & 33 & 33 & 33 & 33 & 38 & 37 \\
\hline 1202.0 & 33 & 33 & 33 & 33 & 34 & 33 & 33 & 33 & 37 & 37 \\
\hline 1207.0 & 32 & 33 & 33 & 33 & 33 & 33 & 33 & 33 & 36 & 37 \\
\hline 1213.0 & 33 & 33 & 33 & 33 & 33 & 33 & 33 & 33 & 36 & 36 \\
\hline 1219.0 & 33 & 33 & 33 & 33 & 33 & 33 & 33 & 33 & 37 & 36 \\
\hline 1225.0 & 33 & 33 & 33 & 33 & 33 & 33 & 33 & 33 & 36 & 36 \\
\hline 1231.0 & 33 & 33 & 33 & 33 & 33 & 33 & 33 & 33 & 36 & 36 \\
\hline 1237.0 & 33 & 33 & 33 & 33 & 33 & 33 & 33 & 33 & 36 & 36 \\
\hline 1242.0 & 33 & 33 & 33 & 33 & 33 & 33 & 33 & 33 & 35 & 36 \\
\hline
\end{tabular}


THERMOCOUPLES AND DISKS AT SPRINKLER AND DETECTOR LOCATIONS - ${ }^{\circ} \mathrm{C}$

\begin{tabular}{llllllllllll}
\hline$\pi M E$ & $T 14$ & $D 15$ & $T 16$ & $D 17$ & $T 18$ & $D 19$ & $T 20$ & $D 21$ & $T 22$ & $D 23$ \\
\hline \hline 1248.0 & 33 & 33 & 33 & 33 & 33 & 33 & 33 & 33 & 34 & 35 \\
1254.0 & 33 & 33 & 33 & 33 & 33 & 33 & 33 & 33 & 35 & 35 \\
1260.0 & 33 & 33 & 33 & 33 & 33 & 33 & 33 & 33 & 34 & 35 \\
1266.0 & 33 & 33 & 33 & 33 & 33 & 33 & 33 & 33 & 34 & 35 \\
1272.0 & 33 & 33 & 33 & 33 & 33 & 33 & 33 & 33 & 34 & 35 \\
1277.0 & 33 & 33 & 33 & 33 & 33 & 33 & 33 & 33 & 34 & 35 \\
1283.0 & 32 & 32 & 33 & 33 & 33 & 33 & 33 & 33 & 35 & 35 \\
1289.0 & 33 & 32 & 33 & 33 & 33 & 33 & 33 & 33 & 35 & 35 \\
1295.0 & 32 & 32 & 33 & 33 & 33 & 33 & 33 & 33 & 33 & 35 \\
1301.0 & 32 & 32 & 33 & 33 & 33 & 33 & 33 & 33 & 34 & 34 \\
1307.0 & 32 & 32 & 33 & 33 & 33 & 33 & 33 & 33 & 34 & 34 \\
1312.0 & 32 & 32 & 33 & 33 & 33 & 33 & 33 & 33 & 34 & 34
\end{tabular}


THERMOCOUPLES AND DISKS AT SPRINKLER AND DETECTOR LOCATIONS - ${ }^{\circ} \mathrm{C}$

\begin{tabular}{|c|c|c|c|c|c|c|c|c|c|c|}
\hline ПМЕ & T24 & $\mathrm{D} 25$ & T26 & $\mathrm{D} 27$ & T28 & D29 & PAN A & PAN B & PAN C & PAN D \\
\hline-54 & 24 & 24 & 24 & 24 & 24 & 24 & 24 & 24 & 24 & 24 \\
\hline-48 & 25 & 24 & 24 & 24 & 24 & 24 & 24 & 24 & 24 & 24 \\
\hline-42 & 25 & 24 & 24 & 24 & 24 & 24 & 24 & 24 & 24 & 24 \\
\hline-37 & 25 & 24 & 24 & 24 & 24 & 24 & 24 & 24 & 24 & 24 \\
\hline-31 & 25 & 24 & 24 & 24 & 24 & 24 & 24 & 24 & 24 & 24 \\
\hline-25 & 25 & 24 & 24 & 24 & 24 & 24 & 24 & 24 & 24 & 24 \\
\hline-19 & 24 & 24 & 24 & 24 & 24 & 24 & 24 & 24 & 24 & 24 \\
\hline-14 & 24 & 24 & 24 & 24 & 24 & 24 & 24 & 24 & 24 & 24 \\
\hline-8 & 25 & 24 & 24 & 24 & 24 & 24 & 24 & 24 & 24 & 24 \\
\hline-2 & 25 & 24 & 24 & 24 & 24 & 24 & 24 & 24 & 24 & 24 \\
\hline 3 & 25 & 24 & 24 & 24 & 24 & 24 & 24 & 24 & 24 & 24 \\
\hline 8 & 25 & 24 & 24 & 24 & 24 & 24 & 24 & 24 & 24 & 24 \\
\hline 14 & 25 & 24 & 24 & 24 & 24 & 24 & 26 & 24 & 24 & 25 \\
\hline 20 & 25 & 25 & 24 & 24 & 24 & 24 & 27 & 24 & 24 & 27 \\
\hline 26 & 25 & 25 & 24 & 24 & 24 & 24 & 28 & 24 & 24 & 27 \\
\hline 31 & 26 & 25 & 26 & 25 & 24 & 24 & 29 & 24 & 24 & 28 \\
\hline 37 & 27 & 25 & 26 & 25 & 24 & 24 & 30 & 24 & 24 & 29 \\
\hline 43 & 27 & 25 & 27 & 25 & 25 & 24 & 31 & 25 & 24 & 29 \\
\hline 49 & 28 & 26 & 27 & 26 & 25 & 24 & 31 & 25 & 25 & 30 \\
\hline 54 & 30 & 26 & 28 & 26 & 26 & 25 & 33 & 26 & 25 & 30 \\
\hline 60 & 30 & 27 & 28 & 26 & 25 & 25 & 34 & 25 & 25 & 31 \\
\hline 66 & 31 & 27 & 29 & 27 & 26 & 25 & 34 & 26 & 26 & 31 \\
\hline 72 & 31 & 27 & 29 & 27 & 27 & 25 & 34 & 27 & 26 & 32 \\
\hline 77 & 31 & 28 & 29 & 27 & 26 & 25 & 35 & 26 & 26 & 32 \\
\hline 83 & 32 & 28 & 30 & 28 & 26 & 25 & 34 & 26 & 26 & 33 \\
\hline
\end{tabular}


CLOSED DOOR PRIVACY CURTAIN TEST

THERMOCOUPLES AND DISKS AT SPRINKLER AND DETECTOR LOCATIONS - ${ }^{\circ} \mathrm{C}$

\begin{tabular}{|c|c|c|c|c|c|c|c|c|c|c|}
\hline TIME & T24 & D25 & T26 & D27 & T28 & D29 & PAN A & PAN B & PAN C & PAN D \\
\hline 89 & 32 & 29 & 30 & 28 & 26 & 25 & 35 & 26 & 26 & 34 \\
\hline 95 & 33 & 29 & 30 & 28 & 27 & 26 & 37 & 27 & 27 & 34 \\
\hline 100 & 34 & 29 & 31 & 29 & 28 & 26 & 37 & 28 & 27 & 35 \\
\hline 106 & 34 & 30 & 32 & 29 & 27 & 26 & 39 & 27 & 27 & 36 \\
\hline 112 & 35 & 30 & 32 & 29 & 28 & 26 & 39 & 28 & 28 & 35 \\
\hline 118 & 35 & 31 & 33 & 30 & 28 & 27 & 40 & 28 & 28 & 36 \\
\hline 123 & 36 & 32 & 33 & 30 & 28 & 27 & 41 & 28 & 28 & 37 \\
\hline 129 & 37 & 32 & 34 & 31 & 28 & 27 & 41 & 28 & 28 & 38 \\
\hline 135 & 38 & 33 & 34 & 31 & 29 & 27 & 42 & 29 & 29 & 38 \\
\hline 141 & 38 & 33 & 35 & 32 & 28 & 28 & 42 & 28 & 30 & 38 \\
\hline 146 & 39 & 34 & 35 & 32 & 29 & 28 & 44 & 29 & 30 & 39 \\
\hline 152 & 39 & 34 & 36 & 33 & 29 & 28 & 44 & 29 & 30 & 40 \\
\hline 158 & 41 & 35 & 37 & 33 & 30 & 28 & 45 & 30 & 29 & 41 \\
\hline 164 & 40 & 35 & 37 & 34 & 30 & 29 & 45 & 30 & 30 & 42 \\
\hline 169 & 49 & 36 & 38 & 34 & 31 & 29 & 45 & 31 & 31 & 42 \\
\hline 175 & 41 & 36 & 39 & 35 & 33 & 29 & 46 & 33 & 31 & 44 \\
\hline 181 & 42 & 37 & 39 & 35 & 33 & 30 & 47 & 33 & 32 & 45 \\
\hline 187 & 43 & 38 & 40 & 36 & 35 & 31 & 48 & 35 & 32 & 46 \\
\hline 192 & 43 & 38 & 40 & 36 & 35 & 31 & 50 & 35 & 32 & 46 \\
\hline 198 & 45 & 39 & 41 & 37 & 34 & 31 & 50 & 34 & 32 & 47 \\
\hline 204 & 44 & 39 & 42 & 37 & 33 & 32 & 52 & 33 & 33 & 47 \\
\hline 210 & 45 & 40 & 42 & 38 & 35 & 32 & 52 & 35 & 33 & 48 \\
\hline 215 & 44 & 40 & 43 & 39 & 35 & 32 & 53 & 35 & 33 & 48 \\
\hline 221 & 45 & 40 & 44 & 39 & 35 & 32 & 53 & 35 & 34 & 49 \\
\hline 227 & 46 & 41 & 43 & 39 & 35 & 33 & 52 & 35 & 34 & 49 \\
\hline
\end{tabular}


THERMOCOUPLES AND DISKS AT SPRINKLER AND DETECTOR LOCATIONS - ${ }^{\circ} \mathrm{C}$

\begin{tabular}{|c|c|c|c|c|c|c|c|c|c|c|}
\hline TIME & $T 24$ & D25 & T26 & $D 27$ & T28 & D29 & PAN A & PAN B & PAN C & PAN D \\
\hline 233 & 47 & 42 & 44 & 40 & 36 & 33 & 54 & 36 & 35 & 50 \\
\hline 238 & 48 & 42 & 45 & 40 & 36 & 33 & 55 & 36 & 35 & 51 \\
\hline 244 & 49 & 43 & 45 & 41 & 39 & 34 & 57 & 39 & 35 & 51 \\
\hline 250 & 51 & 44 & 45 & 41 & 40 & 34 & 57 & 40 & 35 & 51 \\
\hline 256 & 50 & 44 & 45 & 42 & 40 & 34 & 57 & 40 & 36 & 52 \\
\hline 261 & 49 & 45 & 46 & 42 & 40 & 35 & 58 & 40 & 37 & 53 \\
\hline 267 & 50 & 45 & 47 & 43 & 38 & 35 & 58 & 38 & 36 & 53 \\
\hline 273 & 51 & 45 & 47 & 43 & 39 & 35 & 59 & 39 & 37 & 54 \\
\hline 279 & 50 & 46 & 47 & 43 & 39 & 35 & 58 & 39 & 37 & 54 \\
\hline 284 & 52 & 46 & 48 & 44 & 40 & 36 & 58 & 40 & 37 & 55 \\
\hline 290 & 54 & 47 & 48 & 44 & 41 & 36 & 60 & 41 & 38 & 56 \\
\hline 296 & 54 & 47 & 49 & 45 & 43 & 37 & 61 & 43 & 37 & 56 \\
\hline 301 & 55 & 48 & 50 & 45 & 43 & 37 & 62 & 43 & 38 & 57 \\
\hline 307 & 54 & 48 & 50 & 46 & 43 & 38 & 63 & 43 & 38 & 56 \\
\hline 313 & 56 & 49 & 51 & 46 & 40 & 38 & 65 & 40 & 38 & 57 \\
\hline 319 & 56 & 50 & 51 & 47 & 42 & 38 & 64 & 42 & 40 & 59 \\
\hline 324 & 57 & 50 & 53 & 48 & 44 & 39 & 67 & 44 & 41 & 60 \\
\hline 330 & 57 & 51 & 53 & 48 & 44 & 39 & 66 & 44 & 41 & 62 \\
\hline 336 & 58 & 51 & 53 & 49 & 46 & 40 & 70 & 46 & 41 & 63 \\
\hline 342 & 60 & 52 & 53 & 49 & 47 & 41 & 69 & 47 & 41 & 61 \\
\hline 348 & 61 & 53 & 55 & 50 & 46 & 41 & 70 & 46 & 43 & 62 \\
\hline 353 & 60 & 53 & 55 & 50 & 48 & 42 & 69 & 48 & 42 & 63 \\
\hline 359 & 60 & 54 & 56 & 51 & 48 & 42 & 69 & 48 & 43 & 66 \\
\hline 365 & 61 & 54 & 56 & 51 & 48 & 43 & 71 & 48 & 44 & 65 \\
\hline 371 & 63 & 55 & 58 & 52 & 49 & 43 & 69 & 49 & 43 & 66 \\
\hline
\end{tabular}


CLOSED DOOR PRIVACY CURTAIN TEST

THERMOCOUPLES AND DISKS AT SPRINKLER AND DETECTOR LOCATIONS - ${ }^{\circ} \mathrm{C}$

\begin{tabular}{|c|c|c|c|c|c|c|c|c|c|c|}
\hline TME & T24 & $\mathrm{D} 25$ & T26 & D27 & T28 & $\mathrm{D} 29$ & PAN A & PAN B & PAN C & PAN D \\
\hline 376 & 63 & 55 & 59 & 53 & 48 & 44 & 73 & 48 & 42 & 65 \\
\hline 382 & 64 & 56 & 60 & 54 & 51 & 45 & 72 & 51 & 44 & 67 \\
\hline 388 & 65 & 57 & 60 & 54 & 49 & 45 & 75 & 49 & 44 & 68 \\
\hline 394 & 65 & 57 & 60 & 55 & 49 & 45 & 73 & 49 & 44 & 69 \\
\hline 399 & 67 & 58 & 59 & 55 & 51 & 46 & 76 & 51 & 43 & 70 \\
\hline 405 & 67 & 59 & 60 & 55 & 50 & 47 & 79 & 50 & 48 & 70 \\
\hline 411 & 65 & 59 & 61 & 56 & 51 & 47 & 79 & 51 & 47 & 70 \\
\hline 416 & 65 & 59 & 61 & 56 & 51 & 47 & 80 & 51 & 47 & 71 \\
\hline 422 & 68 & 60 & 63 & 57 & 50 & 48 & 79 & 50 & 48 & 71 \\
\hline 428 & 68 & 61 & 64 & 58 & 51 & 49 & 83 & 51 & 47 & 72 \\
\hline 434 & 70 & 61 & 64 & 58 & 52 & 49 & 85 & 52 & 49 & 73 \\
\hline 439 & 72 & 62 & 65 & 59 & 52 & 50 & 86 & 52 & 48 & 75 \\
\hline 445 & 73 & 64 & 67 & 60 & 54 & 51 & 87 & 54 & 50 & 74 \\
\hline 451 & 74 & 65 & 69 & 61 & 54 & 51 & 86 & 54 & 50 & 78 \\
\hline 457 & 76 & 66 & 68 & 62 & 57 & 52 & 85 & 57 & 49 & $\pi$ \\
\hline 462 & 73 & 66 & 70 & 63 & 57 & 52 & 86 & 57 & 49 & $\pi$ \\
\hline 468 & 76 & 67 & 70 & 63 & 56 & 53 & 86 & 56 & 51 & 78 \\
\hline 474 & 76 & 67 & 70 & 64 & 55 & 53 & 83 & 55 & 50 & $\pi$ \\
\hline 480 & 76 & 68 & 6 & 64 & 56 & 53 & 85 & 56 & 50 & $\pi$ \\
\hline 485 & 75 & 68 & 70 & 65 & 56 & 54 & 84 & 56 & 52 & 75 \\
\hline 491 & 75 & 68 & 71 & 65 & 55 & 53 & 82 & 55 & 52 & $\pi$ \\
\hline 497 & 75 & 68 & 70 & 66 & 54 & 54 & 83 & 54 & 52 & 76 \\
\hline 503 & 77 & 69 & 69 & 65 & 56 & 54 & 83 & 56 & 53 & 76 \\
\hline 508 & 75 & 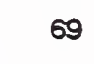 & 69 & 66 & 55 & 54 & 84 & 55 & 51 & 76 \\
\hline 514 & 75 & 69 & 69 & 65 & 56 & 54 & 85 & 56 & 52 & 77 \\
\hline
\end{tabular}


THERMOCOUPLES AND DISKS AT SPRINKLER AND DETECTOR LOCATIONS - ${ }^{\circ} \mathrm{C}$

\begin{tabular}{|c|c|c|c|c|c|c|c|c|c|c|}
\hline TIME & $T 24$ & $\mathrm{D} 25$ & T26 & $\mathrm{D} 27$ & T28 & $\mathrm{D} 29$ & PAN A & PAN B & PAN C & PAN D \\
\hline 520 & 75 & 69 & 69 & 65 & 58 & 55 & 84 & 58 & 52 & 77 \\
\hline 526 & 77 & 69 & 70 & 66 & 59 & 55 & 84 & 59 & 53 & 78 \\
\hline 531 & 76 & 69 & 70 & 66 & 57 & 55 & 86 & 57 & 52 & 77 \\
\hline 537 & 78 & 70 & 71 & 66 & 56 & 55 & 88 & 56 & 52 & 81 \\
\hline 543 & $\pi$ & 70 & 72 & 67 & 58 & 55 & 89 & 58 & 52 & 80 \\
\hline 549 & 78 & 70 & 72 & 67 & 57 & 56 & 89 & 57 & 53 & 80 \\
\hline 554 & 82 & 72 & 72 & 67 & 59 & 56 & 89 & 59 & 54 & 81 \\
\hline 560 & 79 & 72 & 74 & 68 & 58 & 57 & 92 & 58 & 52 & 83 \\
\hline 566 & 84 & 73 & 74 & 68 & 60 & 57 & 91 & 60 & 55 & 81 \\
\hline 572 & 79 & 73 & 74 & 69 & 61 & 57 & 91 & 61 & 54 & 81 \\
\hline 577 & 80 & 73 & 74 & 69 & 59 & 58 & 91 & 59 & 55 & 84 \\
\hline 583 & 83 & 74 & 76 & 70 & 58 & 58 & 93 & 58 & 54 & 85 \\
\hline 589 & 83 & 74 & 75 & 70 & 60 & 58 & 95 & 60 & 56 & 85 \\
\hline 595 & 81 & 74 & 77 & 71 & 60 & 59 & 94 & 60 & 56 & 86 \\
\hline 600 & 84 & 75 & 79 & 71 & 61 & 59 & 96 & 61 & 57 & 87 \\
\hline 606 & 83 & 76 & 77 & 72 & 60 & 59 & 97 & 60 & 58 & 88 \\
\hline 612 & 86 & 76 & 79 & 72 & 60 & 60 & 99 & 60 & 56 & 87 \\
\hline 618 & 89 & 78 & 81 & 73 & 61 & 60 & 100 & 61 & 59 & 86 \\
\hline 623 & 89 & 78 & 81 & 74 & 62 & 61 & 102 & 62 & 59 & 88 \\
\hline 629 & 88 & 79 & 82 & 75 & 65 & 61 & 102 & 65 & 60 & 89 \\
\hline 635 & 90 & 80 & 84 & 76 & 63 & 62 & 101 & 63 & 59 & 90 \\
\hline 641 & 90 & 80 & 84 & 76 & 63 & 62 & 104 & 63 & 60 & 94 \\
\hline 646 & 92 & 81 & 85 & 77 & 66 & 63 & 105 & 66 & 62 & 94 \\
\hline 652 & 93 & 83 & 85 & 78 & 66 & 63 & 107 & 66 & 62 & 95 \\
\hline 658 & 93 & 83 & 86 & 79 & 65 & 64 & 109 & 65 & 65 & 98 \\
\hline
\end{tabular}


THERMOCOUPLES AND DISKS AT SPRINKLER AND DETECTOR LOCATIONS - ${ }^{\circ} \mathrm{C}$

\begin{tabular}{|c|c|c|c|c|c|c|c|c|c|c|}
\hline TME & T24 & $\mathrm{D} 25$ & T26 & $\mathrm{D} 27$ & $T 28$ & $\mathrm{D} 29$ & PAN A & PAN B & PAN C & PAN D \\
\hline 664 & 95 & 84 & 86 & 80 & 66 & 65 & 111 & 66 & 65 & 98 \\
\hline 669 & 94 & 85 & 88 & 80 & 68 & 65 & 112 & 68 & 64 & 101 \\
\hline 675 & 98 & 86 & 87 & 81 & 69 & 66 & 119 & 69 & 65 & 104 \\
\hline 681 & 100 & 87 & 91 & 82 & 69 & 67 & 116 & 69 & 68 & 104 \\
\hline 687 & 105 & 89 & 92 & 83 & 70 & 68 & 119 & 70 & 68 & 105 \\
\hline 692 & 101 & 90 & 93 & 84 & 70 & 68 & 121 & 70 & 68 & 106 \\
\hline 698 & 104 & 91 & 96 & 85 & 72 & 69 & 123 & 72 & 70 & 109 \\
\hline 704 & 105 & 92 & 96 & 87 & 72 & 70 & 126 & 72 & 68 & 111 \\
\hline 710 & 104 & 93 & 99 & 88 & 74 & 71 & 127 & 74 & 72 & 112 \\
\hline 716 & 108 & 95 & 100 & 90 & 75 & 72 & 130 & 75 & 72 & 114 \\
\hline 721 & 108 & 96 & 102 & 91 & $\pi 7$ & 73 & 130 & $\pi$ & 72 & 114 \\
\hline 727 & 113 & 98 & 102 & 92 & 75 & 74 & 132 & 75 & 74 & 113 \\
\hline 733 & 115 & 99 & 103 & 93 & $\pi$ & 75 & 133 & $\pi$ & 73 & 117 \\
\hline 739 & 116 & 101 & 104 & 94 & 81 & 76 & 133 & 81 & 75 & 118 \\
\hline 745 & 117 & 102 & 107 & 96 & 79 & $\pi 7$ & 136 & 79 & 73 & 118 \\
\hline 751 & 114 & 103 & 109 & 97 & 82 & 78 & 135 & 82 & 76 & 120 \\
\hline 757 & 118 & 105 & 106 & 98 & 81 & 79 & 137 & 81 & 78 & 119 \\
\hline 763 & 96 & 104 & 107 & 97 & 26 & 28 & 42 & 26 & 78 & 61 \\
\hline 769 & 75 & 96 & 71 & 91 & 30 & 31 & 39 & 30 & $\pi 7$ & 46 \\
\hline 775 & 71 & 89 & 59 & 82 & 29 & 30 & 37 & 29 & 68 & 38 \\
\hline 780 & 62 & 82 & 51 & 75 & 28 & 29 & 36 & 28 & 59 & 44 \\
\hline 786 & 56 & 76 & 49 & 69 & 29 & 30 & 35 & 29 & 57 & 43 \\
\hline 792 & 44 & 68 & 45 & 63 & 29 & 31 & 35 & 29 & 54 & 36 \\
\hline 798 & 44 & 62 & 43 & 58 & 29 & 30 & 35 & 29 & 52 & 41 \\
\hline 804 & 44 & 58 & 41 & 54 & 29 & 31 & 34 & 29 & 49 & 39 \\
\hline
\end{tabular}


CLOSED DOOR PRIVACY CURTAIN TEST

THERMOCOUPLES AND DISKS AT SPRINKLER AND DETECTOR LOCATIONS $\cdot{ }^{\circ} \mathrm{C}$

\begin{tabular}{|c|c|c|c|c|c|c|c|c|c|c|}
\hline TIME & $T 24$ & 025 & T26 & 027 & T28 & 029 & PAN A & PAN B & PAN C & PAN D \\
\hline 810 & 42 & 54 & 42 & 51 & 29 & 30 & 34 & 29 & 48 & 36 \\
\hline 816 & 41 & 51 & 40 & 47 & 28 & 30 & 34 & 28 & 47 & 38 \\
\hline 822 & 42 & 48 & 39 & 45 & 28 & 30 & 34 & 28 & 45 & 39 \\
\hline 827 & 41 & 47 & 38 & 43 & 28 & 29 & 34 & 28 & 44 & 38 \\
\hline 833 & 39 & 45 & 39 & 42 & 28 & 30 & 33 & 28 & 42 & 37 \\
\hline 839 & 39 & 43 & 38 & 41 & 28 & 29 & 33 & 28 & 42 & 38 \\
\hline 845 & 39 & 43 & 38 & 40 & 28 & 30 & 33 & 28 & 41 & 40 \\
\hline 851 & 39 & 42 & 38 & 39 & 28 & 29 & 33 & 28 & 40 & 38 \\
\hline 857 & 40 & 42 & 38 & 39 & 28 & 29 & 33 & 28 & 40 & 37 \\
\hline 862 & 39 & 41 & 38 & 38 & 29 & 30 & 33 & 29 & 39 & 36 \\
\hline 868 & 38 & 41 & 37 & 38 & 29 & 30 & 33 & 29 & 39 & 35 \\
\hline 874 & 38 & 40 & 37 & 37 & 29 & 30 & 33 & 29 & 39 & 40 \\
\hline 880 & 43 & 41 & 39 & 37 & 29 & 30 & 33 & 29 & 38 & 33 \\
\hline 886 & 39 & 40 & 37 & 37 & 29 & 30 & 33 & 29 & 38 & 40 \\
\hline 892 & 38 & 40 & 37 & 36 & 29 & 30 & 33 & 29 & 38 & 37 \\
\hline 898 & 39 & 40 & 36 & 36 & 29 & 29 & 33 & 29 & 38 & 35 \\
\hline 904 & 37 & 39 & 36 & 36 & 28 & 29 & 33 & 28 & 38 & 36 \\
\hline 909 & 37 & 39 & 36 & 35 & 28 & 29 & 32 & 28 & 38 & 37 \\
\hline 915 & 38 & 39 & 36 & 35 & 28 & 29 & 32 & 28 & 37 & 36 \\
\hline 921 & 38 & 38 & 36 & 35 & 28 & 29 & 32 & 28 & 37 & 36 \\
\hline 927 & 39 & 39 & 36 & 35 & 28 & 29 & 32 & 28 & 37 & 34 \\
\hline 933 & 37 & 38 & 35 & 35 & 28 & 29 & 32 & 28 & 37 & 35 \\
\hline 939 & 40 & 39 & 36 & 35 & 28 & 29 & 32 & 28 & 37 & 33 \\
\hline 945 & 38 & 38 & 36 & 35 & 28 & 29 & 32 & 28 & 36 & 33 \\
\hline 951 & 36 & 38 & 36 & 35 & 28 & 29 & 32 & 28 & 36 & 37 \\
\hline
\end{tabular}




\begin{tabular}{|c|c|c|c|c|c|c|c|c|c|c|}
\hline \multicolumn{11}{|c|}{ THERMOCOUPLES AND DISKS AT SPRINKLER AND DETECTOR LOCATIONS - ${ }^{\circ} \mathrm{C}$} \\
\hline TIME & T24 & D25 & T26 & $\mathrm{D} 27$ & T28 & D29 & PAN A & PAN B & PAN C & PAN D \\
\hline 957 & 40 & 38 & 36 & 35 & 29 & 29 & 32 & 29 & 36 & 34 \\
\hline 962 & 40 & 37 & 35 & 34 & 30 & 31 & 32 & 30 & 36 & 36 \\
\hline 968 & 39 & 38 & 36 & 35 & 31 & 32 & 33 & 31 & 36 & 36 \\
\hline 974 & 40 & 38 & 38 & 35 & 32 & 32 & 33 & 32 & 36 & 35 \\
\hline 980 & 40 & 38 & 36 & 35 & 32 & 32 & 33 & 32 & 36 & 33 \\
\hline 986 & 36 & 37 & 35 & 35 & 32 & 33 & 33 & 32 & 36 & 34 \\
\hline 992 & 35 & 37 & 35 & 35 & 32 & 33 & 33 & 32 & 36 & 33 \\
\hline 997 & 35 & 36 & 35 & 35 & 33 & 33 & 33 & 33 & 36 & 34 \\
\hline 1003 & 34 & 36 & 34 & 34 & 33 & 33 & 33 & 33 & 36 & 34 \\
\hline 1009 & 35 & 35 & 34 & 34 & 33 & 33 & 33 & 33 & 36 & 34 \\
\hline 1015 & 35 & 35 & 34 & 34 & 33 & 33 & 33 & 33 & 36 & 34 \\
\hline 1021 & 35 & 35 & 35 & 34 & 33 & 33 & 34 & 33 & 35 & 34 \\
\hline 1027 & 34 & 35 & 34 & 34 & 33 & 33 & 33 & 33 & 35 & 34 \\
\hline 1032 & 34 & 35 & 34 & 34 & 33 & 33 & 33 & 33 & 35 & 34 \\
\hline 1038 & 34 & 35 & 34 & 34 & 33 & 33 & 33 & 33 & 35 & 34 \\
\hline 1044 & 35 & 35 & 34 & 34 & 33 & 33 & 34 & 33 & 35 & 33 \\
\hline 1050 & 34 & 34 & 34 & 34 & 33 & 33 & 33 & 33 & 35 & 34 \\
\hline 1056 & 34 & 34 & 34 & 34 & 33 & 33 & 33 & 33 & 34 & 34 \\
\hline 1062 & 35 & 34 & 34 & 34 & 33 & 33 & 33 & 33 & 34 & 34 \\
\hline 1067 & 34 & 34 & 34 & 34 & 33 & 33 & 33 & 33 & 34 & 34 \\
\hline 1073 & 34 & 34 & 34 & 34 & 33 & 33 & 33 & 33 & 34 & 34 \\
\hline 1079 & 34 & 34 & 34 & 34 & 33 & 33 & 33 & 33 & 34 & 34 \\
\hline 1085 & 34 & 34 & 34 & 34 & 33 & 33 & 33 & 33 & 34 & 34 \\
\hline 1091 & 34 & 34 & 34 & 34 & 33 & 33 & 33 & 33 & 34 & 33 \\
\hline 1097 & 34 & 34 & - 34 & 34 & 33 & 33 & 33 & 33 & 34 & 33 \\
\hline
\end{tabular}


CLOSED DOOR PRIVACY CURTAIN TEST

THERMOCOUPLES AND DISKS AT SPRINKLER AND DETECTOR LOCATIONS - ${ }^{\circ} \mathrm{C}$

\begin{tabular}{|c|c|c|c|c|c|c|c|c|c|c|}
\hline TIME & T24 & D25 & T26 & D27 & T28 & D29 & PAN A & PAN B & PAN C & PAN D \\
\hline 1102 & 34 & 34 & 34 & 34 & 33 & 33 & 33 & 33 & 34 & 33 \\
\hline 1108 & 34 & 34 & 34 & 34 & 33 & 33 & 33 & 33 & 34 & 34 \\
\hline 1114 & 34 & 34 & 34 & 34 & 33 & 33 & 33 & 33 & 34 & 33 \\
\hline 1120 & 34 & 34 & 34 & 34 & 33 & 33 & 33 & 33 & 34 & 33 \\
\hline 1126 & 34 & 34 & 34 & 34 & 33 & 33 & 33 & 33 & 34 & 33 \\
\hline 1132 & 34 & 34 & 34 & 34 & 33 & 33 & 33 & 33 & 34 & 33 \\
\hline 1137 & 34 & 34 & 34 & 34 & 33 & 33 & 33 & 33 & 33 & 33 \\
\hline 1143 & 34 & 34 & 34 & 34 & 33 & 33 & 33 & 33 & 33 & 33 \\
\hline 1149 & 34 & 34 & 34 & 34 & 33 & 33 & 33 & 33 & 33 & 33 \\
\hline$\$ 155$ & 34 & 34 & 34 & 34 & 33 & 33 & 33 & 33 & 34 & 33 \\
\hline 1161 & 34 & 34 & 34 & 34 & 33 & 33 & 33 & 33 & 33 & 33 \\
\hline 1167 & 34 & 34 & 34 & 34 & 33 & 33 & 33 & 33 & 33 & 33 \\
\hline 1172 & 34 & 34 & 34 & 33 & 33 & 33 & 33 & 33 & 33 & 33 \\
\hline 1178 & 34 & 34 & 33 & 33 & 33 & 33 & 33 & 33 & 33 & 33 \\
\hline 1184 & 34 & 34 & 34 & 34 & 33 & 33 & 33 & 33 & 32 & 33 \\
\hline 1190 & 34 & 34 & 34 & 34 & 33 & 33 & 33 & 33 & 32 & 33 \\
\hline 1196 & 34 & 34 & 33 & 33 & 33 & 33 & 33 & 33 & 33 & 33 \\
\hline 1202 & 34 & 34 & 33 & 33 & 33 & 33 & 33 & 33 & 33 & 33 \\
\hline 1207 & 34 & 34 & 33 & 33 & 33 & 33 & 33 & 33 & 33 & 33 \\
\hline 1213 & 34 & 34 & 33 & 33 & 33 & 33 & 33 & 33 & 33 & 33 \\
\hline 1219 & 33 & 33 & 33 & 33 & 33 & 33 & 33 & 33 & 33 & 33 \\
\hline 1225 & 34 & 33 & 33 & 33 & 33 & 33 & 33 & 33 & 33 & 33 \\
\hline 1231 & 33 & 33 & 33 & 33 & 33 & 33 & 33 & 33 & 32 & 33 \\
\hline 1237 & 33 & 33 & 33 & 33 & 33 & 33 & 33 & 33 & 32 & 33 \\
\hline 1242 & 33 & 33 & 33 & 33 & 33 & 33 & 33 & 33 & 32 & 33 \\
\hline
\end{tabular}


CLOSED DOOR PRIVACY CURTAIN TEST

THERMOCOUPLES AND DISKS AT SPRINKLER AND DETECTOR LOCATIONS - ${ }^{\circ} \mathrm{C}$

\begin{tabular}{llllllllllll}
\hline TME & T24 & D25 & T26 & D27 & T28 & D29 & PAN A & PAN B & PAN C & PAN D \\
\hline 1248 & 33 & 33 & 33 & 33 & 33 & 33 & 33 & 33 & 32 & 33 \\
1254 & 33 & 33 & 33 & 33 & 33 & 33 & 33 & 33 & 32 & 33 \\
1260 & 33 & 33 & 33 & 33 & 33 & 33 & 33 & 33 & 32 & 33 \\
1266 & 33 & 33 & 33 & 33 & 33 & 33 & 33 & 33 & 32 & 33 \\
1272 & 33 & 33 & 33 & 33 & 33 & 33 & 33 & 33 & 32 & 33 \\
1277 & 33 & 33 & 33 & 33 & 33 & 33 & 33 & 33 & 32 & 33 \\
1283 & 33 & 33 & 33 & 33 & 33 & 33 & 33 & 33 & 32 & 33 \\
1289 & 33 & 33 & 33 & 33 & 33 & 33 & 33 & 33 & 32 & 33 \\
1295 & 33 & 33 & 33 & 33 & 33 & 33 & 33 & 33 & 32 & 33 \\
1309 & 33 & 33 & 33 & 33 & 33 & 33 & 33 & 33 & 32 & 33 \\
1307 & 33 & 33 & 33 & 33 & 33 & 33 & 33 & 33 & 32 & 33 \\
1312 & 33 & 33 & 33 & 33 & 33 & 33 & 33 & 33 & 32 & 33
\end{tabular}


OPEN DOOR PRIVACY CURTAIN TEST

THERMOCOUPLES AND DISKS AT SPRINKLER AND DETECTOR LOCATIONS - ${ }^{\circ} \mathrm{C}$

\begin{tabular}{|c|c|c|c|c|c|c|c|c|c|c|}
\hline TIME & $\mathrm{T} 14$ & D15 & T16 & D17 & T18 & D19 & $\mathrm{T} 20$ & D21 & T22 & $\mathrm{D} 23$ \\
\hline-42 & 25 & 25 & 25 & 25 & 26 & 25 & 24 & 25 & 25 & 25 \\
\hline-34 & 25 & 25 & 25 & 25 & 26 & 25 & 25 & 25 & 25 & 25 \\
\hline-29 & 25 & 25 & 25 & 25 & 26 & 25 & 25 & 25 & 25 & 25 \\
\hline-23 & 25 & 25 & 25 & 25 & 26 & 25 & 25 & 25 & 25 & 25 \\
\hline-17 & 25 & 25 & 25 & 25 & 26 & 25 & 25 & 25 & 25 & 25 \\
\hline-11 & 25 & 25 & 25 & 25 & 25 & 25 & 25 & 25 & 25 & 25 \\
\hline-6 & 25 & 25 & 25 & 25 & 25 & 25 & 25 & 25 & 25 & 25 \\
\hline-0 & 25 & 25 & 25 & 25 & 26 & 25 & 25 & 25 & 25 & 25 \\
\hline 5 & 25 & 25 & 25 & 25 & 26 & 25 & 25 & 25 & 25 & 25 \\
\hline 10 & 25 & 25 & 28 & 26 & 26 & 25 & 25 & 25 & 28 & 25 \\
\hline 16 & 26 & 25 & 31 & 27 & 26 & 25 & 26 & 25 & 28 & 26 \\
\hline 22 & 28 & 25 & 32 & 28 & 26 & 25 & 28 & 26 & 29 & 26 \\
\hline 28 & 28 & 26 & 33 & 28 & 28 & 26 & 28 & 26 & 30 & 27 \\
\hline 33 & 29 & 26 & 34 & 29 & 29 & 26 & 29 & 26 & 30 & 27 \\
\hline 39 & 29 & 27 & 36 & 30 & 29 & 27 & 30 & 27 & 32 & 28 \\
\hline 45 & 30 & 27 & 37 & 31 & 30 & 27 & 30 & 27 & 32 & 29 \\
\hline 50 & 31 & 27 & 37 & 32 & 32 & 28 & 31 & 28 & 34 & 29 \\
\hline 56 & 31 & 28 & 38 & 33 & 32 & 28 & 32 & 29 & 35 & 30 \\
\hline 62 & 31 & 28 & 41 & 35 & 32 & 28 & 33 & 29 & 35 & 31 \\
\hline 68 & 33 & 29 & 42 & 36 & 33 & 29 & 33 & 30 & 36 & 32 \\
\hline 73 & 34 & 30 & 44 & 37 & 34 & 30 & 34 & 30 & 36 & 32 \\
\hline 79 & 35 & 30 & 44 & 38 & 35 & 30 & 35 & 31 & 37 & 33 \\
\hline 85 & 37 & 31 & 45 & 39 & 35 & 31 & 35 & 31 & 38 & 34 \\
\hline 91 & 37 & 32 & 45 & 40 & 35 & 31 & 35 & 32 & 39 & 34 \\
\hline 97 & 37 & 33 & 47 & 41 & 36 & 31 & 37 & 32 & 38 & 35 \\
\hline
\end{tabular}


OPEN DOOR PRIVACY CURTAIN TEST

THERMOCOUPLES AND DISKS AT SPRINKLER AND DETECTOR LOCATIONS - ${ }^{\circ} \mathrm{C}$

\begin{tabular}{|c|c|c|c|c|c|c|c|c|c|c|}
\hline TIME & T14 & D15 & T16 & D17 & $T 18$ & D19 & T20 & D21 & T22 & D23 \\
\hline 102 & 39 & 33 & 48 & 42 & 37 & 32 & 36 & 33 & 40 & 36 \\
\hline 108 & 39 & 34 & 48 & 43 & 37 & 32 & 37 & 33 & 41 & 36 \\
\hline 114 & 40 & 35 & 47 & 44 & 38 & 33 & 38 & 34 & 43 & 37 \\
\hline 119 & 40 & 35 & 48 & 44 & 38 & 33 & 39 & 35 & 42 & 38 \\
\hline 125 & 41 & 36 & 49 & 45 & 39 & 34 & 39 & 35 & 43 & 39 \\
\hline 131 & 42 & 37 & 49 & 46 & 40 & 34 & 39 & 35 & 44 & 39 \\
\hline 137 & 42 & 37 & 52 & 47 & 41 & 35 & 41 & 36 & 45 & 40 \\
\hline 142 & 43 & 38 & 53 & 48 & 40 & 35 & 41 & 37 & 44 & 41 \\
\hline 148 & 44 & 39 & 53 & 49 & 41 & 36 & 42 & 37 & 44 & 41 \\
\hline 154 & 45 & 40 & 55 & 50 & 42 & 37 & 43 & 38 & 47 & 42 \\
\hline 160 & 46 & 40 & 58 & 51 & 42 & 37 & 43 & 38 & 46 & 43 \\
\hline 165 & 47 & 41 & 57 & 52 & 43 & 37 & 44 & 39 & 49 & 44 \\
\hline 171 & 47 & 42 & 58 & 53 & 43 & 38 & 44 & 40 & 50 & 45 \\
\hline 177 & 47 & 42 & 59 & 54 & 44 & 38 & 46 & 40 & 49 & 45 \\
\hline 182 & 48 & 43 & 58 & 54 & 45 & 39 & 46 & 41 & 50 & 46 \\
\hline 188 & 49 & 44 & 59 & 55 & 44 & 39 & 46 & 42 & 51 & 47 \\
\hline 194 & 49 & 44 & 60 & 56 & 46 & 40 & 46 & 42 & 53 & 47 \\
\hline 200 & 49 & 45 & 61 & 56 & 46 & 40 & 47 & 43 & 51 & 48 \\
\hline 205 & 50 & 45 & 62 & 57 & 46 & 41 & 48 & 43 & 52 & 48 \\
\hline 211 & 51 & 46 & $\varsigma 2$ & 58 & 49 & 42 & 48 & 44 & 53 & 49 \\
\hline 217 & 51 & 46 & 64 & 58 & 49 & 43 & 49 & 44 & 58 & 50 \\
\hline 223 & 52 & 47 & 65 & 59 & 49 & 43 & 51 & 45 & 56 & 51 \\
\hline 228 & 53 & 48 & 65 & 60 & 50 & 44 & 51 & 46 & 58 & 52 \\
\hline 234 & 54 & 49 & 70 & 62 & 51 & 44 & 52 & 47 & 58 & 53 \\
\hline 240 & 55 & 49 & 67 & 62 & 51 & 45 & 53 & 47 & 59 & 54 \\
\hline
\end{tabular}


OPEN DOOR PRIVACY CURTAIN TEST

THERMOCOUPLES AND DISKS AT SPRINKLER AND DETECTOR LOCATIONS - ${ }^{\circ} \mathrm{C}$

\begin{tabular}{|c|c|c|c|c|c|c|c|c|c|c|}
\hline TIME & $T 14$ & D15 & $T 16$ & D17 & $T 18$ & D19 & T20 & D21 & T22 & D23 \\
\hline 246 & 56 & 50 & 71 & 64 & 52 & 45 & 53 & 48 & 58 & 54 \\
\hline 251 & 57 & 51 & 70 & 65 & 52 & 46 & 52 & 48 & 61 & 55 \\
\hline 257 & 57 & 52 & 70 & 65 & 54 & 47 & 56 & 49 & 61 & 56 \\
\hline 263 & 58 & 52 & 72 & 66 & 55 & 48 & 57 & 50 & 61 & 57 \\
\hline 268 & 59 & 53 & 73 & 67 & 57 & 49 & 56 & 51 & 64 & 58 \\
\hline 274 & 60 & 54 & 74 & 68 & 58 & 49 & 58 & 52 & 63 & 58 \\
\hline 280 & 61 & 54 & 74 & 69 & 57 & 50 & 59 & 52 & 68 & 60 \\
\hline 286 & 63 & 55 & 79 & 71 & 58 & 51 & 59 & 53 & 66 & 61 \\
\hline 291 & 62 & 56 & 77 & 71 & 59 & 51 & 60 & 54 & 67 & 62 \\
\hline 297 & 63 & 57 & 78 & 72 & 60 & 52 & 59 & 54 & 67 & 63 \\
\hline 303 & 64 & 57 & 80 & 74 & 59 & 53 & 61 & 55 & 67 & 63 \\
\hline 308 & 65 & 58 & 79 & 74 & 56 & 52 & 61 & 56 & 68 & 64 \\
\hline 314 & 67 & 59 & 81 & 75 & 60 & 53 & 62 & 56 & 70 & 65 \\
\hline 320 & 68 & 60 & 85 & $\pi 7$ & 62 & 54 & 64 & 57 & 71 & 66 \\
\hline 326 & 69 & 61 & 87 & 78 & 65 & 55 & 64 & 58 & 74 & 67 \\
\hline 331 & 70 & 62 & 86 & 79 & 66 & 56 & 65 & 59 & 75 & 68 \\
\hline 337 & 70 & 63 & 87 & 80 & 66 & 57 & 66 & 59 & 75 & 69 \\
\hline 343 & 72 & 64 & 87 & 82 & 68 & 58 & 68 & 60 & 76 & 70 \\
\hline 349 & 72 & 65 & 89 & 82 & 67 & 59 & 67 & 61 & 76 & 71 \\
\hline 354 & 73 & 66 & 81 & 76 & 68 & 60 & 67 & 61 & 77 & 71 \\
\hline 360 & 72 & 66 & 88 & 78 & 67 & 60 & 69 & 62 & 83 & 73 \\
\hline 366 & 74 & 67 & 91 & 81 & 69 & 61 & 71 & 63 & 80 & 74 \\
\hline 372 & 74 & 68 & 90 & 82 & 70 & 62 & 72 & 64 & 82 & 75 \\
\hline 377 & 76 & 68 & 90 & 84 & 70 & 62 & 73 & 65 & 81 & 76 \\
\hline 383 & $\pi 7$ & 69 & 95 & 86 & 70 & 63 & 72 & 66 & 87 & 78 \\
\hline
\end{tabular}


THERMOCOUPLES AND DISKS AT SPRINKLER AND DETECTOR LOCATIONS - ${ }^{\circ} \mathrm{C}$

\begin{tabular}{|c|c|c|c|c|c|c|c|c|c|c|}
\hline TIME & T14 & D15 & T16 & $\mathrm{D} 17$ & T18 & D19 & T20 & D21 & T22 & D23 \\
\hline 389 & $\pi$ & 70 & 94 & 87 & 74 & 64 & 74 & 67 & 83 & 78 \\
\hline 395 & 78 & 71 & 98 & 89 & 73 & 64 & 74 & 68 & 84 & 79 \\
\hline 400 & 77 & 71 & 98 & 90 & 72 & 65 & 74 & 68 & 85 & 80 \\
\hline 406 & 80 & 72 & 98 & 91 & 74 & 65 & 75 & 69 & 83 & 80 \\
\hline 412 & 81 & 73 & 94 & 91 & 75 & 66 & 78 & 70 & 85 & 80 \\
\hline 417 & 79 & 73 & 96 & 91 & 74 & 66 & 76 & 70 & 84 & 80 \\
\hline 423 & 79 & 74 & 95 & 91 & 73 & 66 & 75 & 70 & 84 & 81 \\
\hline 429 & 78 & 73 & 90 & 90 & 72 & 67 & 74 & 70 & 84 & 81 \\
\hline 435 & 76 & 73 & 91 & 90 & 71 & 66 & 75 & 70 & 79 & 80 \\
\hline 440 & 75 & 73 & 90 & 89 & 73 & 67 & $\pi$ & 71 & 81 & 80 \\
\hline 446 & 76 & 73 & 95 & 89 & 73 & 67 & 74 & 71 & 84 & 81 \\
\hline 452 & 76 & 73 & 95 & 90 & 72 & 67 & 75 & 71 & 83 & 81 \\
\hline 458 & $\pi$ & 73 & 92 & 89 & 72 & 67 & 75 & 71 & 82 & 80 \\
\hline 463 & 78 & 73 & 91 & 89 & 70 & 67 & 75 & 71 & 84 & 81 \\
\hline 469 & 79 & 73 & 95 & 90 & 75 & 67 & 76 & 72 & 87 & 82 \\
\hline 475 & 79 & 73 & 100 & 92 & 76 & 68 & 78 & 72 & 87 & 83 \\
\hline 480 & 80 & 74 & 101 & 93 & 76 & 69 & $\pi$ & 73 & 87 & 83 \\
\hline 486 & 82 & 75 & 103 & 95 & 78 & 69 & 78 & 73 & 87 & 83 \\
\hline 492 & 82 & 75 & 101 & 95 & 79 & 70 & 79 & 74 & 89 & 84 \\
\hline 498 & 82 & 76 & 100 & 96 & 78 & 70 & 80 & 74 & 92 & 86 \\
\hline 504 & 82 & 76 & 104 & 97 & 78 & 71 & 80 & 75 & 93 & 87 \\
\hline 509 & 83 & $\pi$ & 106 & 98 & 76 & 71 & 82 & 76 & 95 & 88 \\
\hline 515 & 85 & $\pi$ & 107 & 99 & 80 & 72 & 83 & 76 & 93 & 89 \\
\hline 521 & 88 & 79 & 110 & 101 & 82 & 73 & 86 & 78 & 95 & 89 \\
\hline 526 & 87 & 79 & 108 & 102 & 84 & 73 & 86 & 78 & 97 & 90 \\
\hline
\end{tabular}


OPEN DOOR PRIVACY CURTAIN TEST

THERMOCOUPLES AND DISKS AT SPRINKLER AND DETECTOR LOCATIONS - ${ }^{\circ} \mathrm{C}$

\begin{tabular}{|c|c|c|c|c|c|c|c|c|c|c|}
\hline TIME & T14 & D15 & T16 & D17 & T18 & D19 & T20 & $\mathrm{D} 21$ & T22 & D23 \\
\hline 532 & 87 & 80 & 110 & 103 & 85 & 74 & 85 & 79 & 95 & 91 \\
\hline 538 & 89 & 81 & 112 & 104 & 85 & 75 & 87 & 80 & 99 & 93 \\
\hline 544 & 70 & 71 & 112 & 105 & 87 & 76 & 91 & 82 & 102 & 94 \\
\hline 549 & 58 & 52 & 111 & 106 & 87 & 77 & 91 & 83 & 103 & 95 \\
\hline 555 & 86 & 51 & 118 & 108 & 90 & 78 & 93 & 84 & 105 & 97 \\
\hline 561 & 90 & 57 & 116 & 110 & 91 & 79 & 92 & 85 & 106 & 99 \\
\hline 567 & 90 & 63 & 122 & 112 & 90 & 80 & 93 & 86 & 107 & 100 \\
\hline 573 & 91 & 69 & 120 & 113 & 92 & 81 & 94 & 86 & 105 & 101 \\
\hline 579 & 92 & 74 & 120 & 114 & 93 & 82 & 99 & 88 & 107 & 102 \\
\hline 584 & 93 & 77 & 122 & 115 & 93 & 83 & 97 & 89 & 111 & 104 \\
\hline 590 & 95 & 81 & 123 & 116 & 95 & 81 & 98 & 90 & 107 & 104 \\
\hline 596 & 95 & 83 & 122 & 117 & 93 & 82 & 98 & 91 & 111 & 105 \\
\hline 602 & 97 & 85 & 122 & 117 & 95 & 84 & 101 & 92 & 114 & 107 \\
\hline 608 & 101 & 87 & 127 & 119 & 96 & 84 & 102 & 93 & 114 & 108 \\
\hline 614 & 102 & 89 & 126 & 120 & 99 & 86 & 104 & 95 & 114 & 109 \\
\hline 619 & 101 & 91 & 130 & 122 & 102 & 87 & 103 & 95 & 121 & 111 \\
\hline 625 & 103 & 92 & 133 & 123 & 100 & 88 & 103 & 96 & 116 & 111 \\
\hline 631 & 106 & 94 & 133 & 125 & 100 & 89 & 106 & 97 & 115 & 112 \\
\hline 637 & 104 & 95 & 134 & 126 & 101 & 90 & 106 & 98 & 119 & 113 \\
\hline 643 & 109 & 97 & 136 & 128 & 99 & 90 & 104 & 98 & 120 & 113 \\
\hline 649 & 107 & 98 & 134 & 128 & 98 & 91 & 105 & 98 & 119 & 114 \\
\hline 655 & 110 & 99 & 137 & 129 & 102 & 92 & 109 & 99 & 123 & 116 \\
\hline 660 & 110 & 100 & 143 & 132 & 104 & 92 & 110 & 101 & 120 & 116 \\
\hline 666 & 108 & 100 & 142 & 133 & 106 & 94 & 109 & 101 & 123 & 117 \\
\hline 672 & 112 & 102 & 142 & 134 & 106 & 94 & 108 & 101 & 123 & 118 \\
\hline
\end{tabular}


OPEN DOOR PRIVACY CURTAIN TEST

\begin{tabular}{|c|c|c|c|c|c|c|c|c|c|c|}
\hline \multicolumn{11}{|c|}{ THERMOCOUPLES AND DISKS AT SPRINKLER AND DETECTOR LOCATIONS - ${ }^{\circ} \mathrm{C}$} \\
\hline TME & T14 & D15 & T16 & D17 & T18 & D19 & T20 & D21 & T22 & $\mathrm{D} 23$ \\
\hline 678 & 115 & 103 & 144 & 136 & 106 & 95 & 110 & 102 & 123 & 119 \\
\hline 684 & 114 & 104 & 145 & 136 & 105 & 95 & 111 & 103 & 124 & 120 \\
\hline 690 & 114 & 105 & 142 & 137 & 108 & 96 & 111 & 104 & 127 & 121 \\
\hline 695 & 117 & 106 & 142 & 138 & 108 & 97 & 109 & 104 & 123 & 121 \\
\hline 701 & 114 & 106 & 143 & 138 & 107 & 97 & 113 & 104 & 130 & 122 \\
\hline 707 & 117 & 107 & 149 & 140 & 106 & 97 & 111 & 105 & 128 & 123 \\
\hline 713 & 117 & 108 & 149 & 141 & 110 & 99 & 116 & 106 & 133 & 125 \\
\hline 719 & 118 & 108 & 145 & 140 & 113 & 100 & 116 & 107 & 130 & 125 \\
\hline 725 & 115 & 108 & 143 & 140 & 110 & 100 & 115 & 108 & 131 & 126 \\
\hline 730 & 114 & 108 & 146 & 141 & 112 & 101 & 116 & 108 & 131 & 127 \\
\hline 736 & 116 & 108 & 149 & 142 & 111 & 101 & 116 & 109 & 129 & 127 \\
\hline 742 & 118 & 109 & 147 & 142 & 111 & 101 & 115 & 109 & 131 & 127 \\
\hline 748 & 117 & 109 & 153 & 145 & 112 & 101 & 117 & 109 & 132 & 128 \\
\hline 754 & 116 & 110 & 138 & 142 & 112 & 102 & 117 & 110 & 130 & 128 \\
\hline 759 & 116 & 109 & 148 & 143 & 115 & 103 & 117 & 110 & 128 & 128 \\
\hline 765 & 119 & 110 & 149 & 143 & 114 & 103 & 116 & 110 & 132 & $\$ 29$ \\
\hline 771 & 120 & 111 & 151 & 144 & 112 & 104 & 120 & 111 & 134 & 130 \\
\hline 777 & 123 & 112 & 148 & 145 & 109 & 103 & 120 & 112 & 137 & 130 \\
\hline 783 & 122 & 112 & 153 & 146 & 113 & 104 & 119 & 112 & 137 & 131 \\
\hline 789 & 123 & 113 & 156 & 148 & 115 & 104 & 123 & 113 & 140 & 133 \\
\hline 794 & 122 & 114 & 153 & 148 & 116 & 105 & 120 & 114 & 140 & 135 \\
\hline 800 & 120 & 114 & 149 & 147 & 112 & 105 & 121 & 114 & 140 & 135 \\
\hline 806 & 122 & 114 & 156 & 148 & 116 & 105 & 125 & 115 & 142 & 136 \\
\hline 812 & 123 & 114 & 151 & 148 & 118 & 106 & 125 & 116 & 142 & 136 \\
\hline 818 & 115 & 111 & 144 & 132 & 40 & 59 & 71 & 77 & 140 & 137 \\
\hline
\end{tabular}


THERMOCOUPLES AND DISKS AT SPRINKLER AND DETECTOR LOCATIONS - ${ }^{\circ} \mathrm{C}$

\begin{tabular}{|c|c|c|c|c|c|c|c|c|c|c|}
\hline TIME & $T 14$ & D15 & $T 16$ & D17 & $T 18$ & D19 & $T_{20}$ & D21 & T22 & $\mathrm{D} 23$ \\
\hline 823 & 38 & 67 & 128 & 82 & 40 & 43 & 93 & 78 & 125 & 134 \\
\hline 829 & 37 & 45 & 104 & 60 & 42 & 44 & 92 & 83 & 118 & 128 \\
\hline 835 & 36 & 39 & 50 & 56 & 35 & 40 & 91 & 86 & 109 & 123 \\
\hline 841 & 35 & 37 & 49 & 50 & 35 & 37 & 86 & 85 & 97 & 115 \\
\hline 847 & 36 & 36 & 49 & 49 & 34 & 36 & 86 & 84 & 93 & 110 \\
\hline 853 & 35 & 35 & 48 & 48 & 34 & 35 & 72 & 80 & 83 & 102 \\
\hline 858 & 34 & 35 & 47 & 47 & 39 & 36 & 66 & 76 & 76 & 96 \\
\hline 864 & 35 & 35 & 45 & 45 & 34 & 34 & 67 & 72 & 73 & 90 \\
\hline 870 & 34 & 35 & 43 & 43 & 33 & 34 & 55 & 68 & 65 & 84 \\
\hline 876 & 33 & 34 & 44 & 44 & 33 & 33 & 49 & 65 & 62 & 79 \\
\hline 882 & 33 & 34 & 44 & 43 & 34 & 33 & 55 & 62 & 63 & 75 \\
\hline 888 & 33 & 34 & 44 & 43 & 34 & 34 & 52 & 56 & 60 & 71 \\
\hline 894 & 33 & 33 & 42 & 42 & 32 & 32 & 55 & 55 & 62 & 69 \\
\hline 899 & 32 & 33 & 43 & 42 & 31 & 32 & 45 & 54 & 59 & 66 \\
\hline 905 & 32 & 33 & 42 & 41 & 33 & 32 & 48 & 54 & 59 & 64 \\
\hline 911 & 32 & 33 & 41 & 41 & 39 & 31 & 48 & 53 & 54 & 61 \\
\hline 917 & 32 & 32 & 40 & 40 & 31 & 31 & 46 & 49 & 55 & 60 \\
\hline 923 & 31 & 32 & 38 & 39 & 31 & 31 & 46 & 47 & 53 & 58 \\
\hline 929 & 31 & 32 & 34 & 36 & 31 & 31 & 48 & 46 & 54 & 57 \\
\hline 935 & 32 & 32 & 34 & 35 & 30 & 31 & 49 & 45 & 48 & 55 \\
\hline 940 & 32 & 32 & 34 & 34 & 31 & 31 & 46 & 44 & 48 & 54 \\
\hline 946 & 31 & 32 & 34 & 34 & 31 & 31 & 39 & 43 & 49 & 53 \\
\hline 952 & 31 & 32 & 33 & 34 & 31 & 31 & 40 & 42 & 50 & 52 \\
\hline 958 & 31 & 31 & 33 & 34 & 39 & 31 & 46 & 43 & 51 & 52 \\
\hline 964 & 31 & 31 & 32 & 33 & 31 & 31 & 39 & 41 & 51 & 52 \\
\hline
\end{tabular}


OPEN DOOR PRIVACY CURTAIN TEST

THERMOCOUPLES AND DISKS AT SPRINKLER AND DETECTOR LOCATIONS - ${ }^{\circ} \mathrm{C}$

\begin{tabular}{|c|c|c|c|c|c|c|c|c|c|c|}
\hline TIME & T14 & D15 & T16 & D17 & T18 & D19 & T20 & $\mathrm{D} 21$ & T22 & D23 \\
\hline 970 & 31 & 31 & 32 & 33 & 31 & 31 & 42 & 41 & 48 & 51 \\
\hline 976 & 31 & 31 & 32 & 33 & 30 & 30 & 40 & 40 & 50 & 50 \\
\hline 982 & 31 & 31 & 32 & 32 & 30 & 30 & 39 & 39 & 48 & 50 \\
\hline 987 & 31 & 31 & 32 & 32 & 30 & 30 & 37 & 38 & 45 & 49 \\
\hline 993 & 31 & 31 & 32 & 32 & 31 & 31 & 41 & 38 & 47 & 48 \\
\hline 999 & 31 & 31 & 33 & 32 & 31 & 31 & 42 & 38 & 46 & 48 \\
\hline 1005 & 32 & 31 & 33 & 33 & 32 & 32 & 40 & 38 & 47 & 47 \\
\hline 1011 & 32 & 32 & 33 & 33 & 33 & 32 & 38 & 37 & 42 & 47 \\
\hline 1017 & 32 & 32 & 34 & 33 & 33 & 33 & 36 & 37 & 45 & 46 \\
\hline 1023 & 33 & 32 & 34 & 34 & 34 & 34 & 35 & 36 & 43 & 45 \\
\hline 1028 & 34 & 33 & 35 & 34 & 35 & 35 & 36 & 36 & 44 & 45 \\
\hline 1034 & 34 & 33 & 35 & 35 & 35 & 35 & 36 & 36 & 42 & 45 \\
\hline 1040 & 35 & 34 & 35 & 35 & 35 & 35 & 36 & 36 & 42 & 44 \\
\hline 1046 & 35 & 34 & 36 & 36 & 36 & 36 & 35 & 35 & 42 & 44 \\
\hline 1052 & 35 & 35 & 36 & 36 & 36 & 36 & 35 & 35 & 43 & 43 \\
\hline 1058 & 35 & 35 & 36 & 36 & 36 & 36 & 36 & 36 & 42 & 43 \\
\hline 1064 & 35 & 35 & 37 & 37 & 36 & 36 & 36 & 36 & 41 & 42 \\
\hline 1069 & 35 & 35 & 37 & 37 & 36 & 37 & 36 & 36 & 41 & 42 \\
\hline 1075 & 36 & 35 & 37 & 37 & 37 & 37 & 36 & 36 & 41 & 42 \\
\hline 1081 & 37 & 36 & 37 & 37 & 36 & 37 & 36 & 36 & 42 & 42 \\
\hline 1087 & 36 & 36 & 37 & 37 & 37 & 37 & 38 & 37 & 41 & 42 \\
\hline 1093 & 36 & 36 & 37 & 37 & 36 & 37 & 37 & 37 & 40 & 42 \\
\hline 1099 & 37 & 36 & 37 & 37 & 36 & 37 & 37 & 37 & 40 & 41 \\
\hline 9104 & 36 & 36 & 37 & 38 & 37 & 37 & 36 & 37 & 41 & 41 \\
\hline 1110 & 37 & 36 & 37 & 38 & 37 & 37 & 36 & 37 & 40 & 41 \\
\hline
\end{tabular}


THERMOCOUPLES AND DISKS AT SPRINKLER AND DETECTOR LOCATIONS - ${ }^{\circ} \mathrm{C}$

\begin{tabular}{|c|c|c|c|c|c|c|c|c|c|c|}
\hline TIME & $\mathrm{T} 14$ & D15 & T16 & D17 & T18 & D19 & T20 & $\mathrm{D} 21$ & T22 & $\mathrm{D} 23$ \\
\hline 1116 & 37 & 36 & 37 & 38 & 37 & 37 & 36 & 36 & 39 & 40 \\
\hline 1122 & 37 & 37 & 37 & 38 & 37 & 37 & 36 & 36 & 40 & 40 \\
\hline 1128 & 36 & 37 & 38 & 38 & 37 & 37 & 36 & 36 & 39 & 40 \\
\hline 1134 & 36 & 37 & 38 & 38 & 37 & 37 & 37 & 37 & 40 & 40 \\
\hline 1139 & 36 & 36 & 38 & 38 & 37 & 37 & 37 & 37 & 39 & 40 \\
\hline 1145 & 37 & 37 & 38 & 38 & 37 & 38 & 37 & 37 & 40 & 40 \\
\hline 1151 & 37 & 37 & 38 & 38 & 37 & 38 & 37 & 37 & 38 & 39 \\
\hline 1157 & 37 & 37 & 38 & 38 & 38 & 38 & 37 & 37 & 39 & 39 \\
\hline 1163 & 37 & 37 & 38 & 38 & 38 & 38 & 37 & 37 & 39 & 39 \\
\hline 1169 & 37 & 37 & 38 & 38 & 37 & 37 & 36 & 36 & 39 & 39 \\
\hline 1175 & 37 & 37 & 38 & 38 & 37 & 37 & 36 & 36 & 39 & 39 \\
\hline 1180 & 37 & 37 & 38 & 38 & 37 & 37 & 37 & 37 & 38 & 39 \\
\hline 1186 & 37 & 37 & 38 & 38 & 37 & 37 & 37 & 37 & 39 & 39 \\
\hline 1192 & 37 & 37 & 38 & 38 & 38 & 38 & 37 & 37 & 38 & 38 \\
\hline 1198 & 37 & 37 & 38 & 38 & 37 & 38 & 37 & 37 & 38 & 38 \\
\hline 1204 & 37 & 37 & 38 & 38 & 37 & 38 & 37 & 37 & 38 & 38 \\
\hline$i 210$ & 38 & 37 & 38 & 38 & 38 & 38 & 37 & 37 & 38 & 38 \\
\hline 1215 & 38 & 37 & 38 & 38 & 38 & 38 & 37 & 37 & 38 & 38 \\
\hline 1221 & 38 & 37 & 38 & 38 & 37 & 38 & 37 & 37 & 38 & 38 \\
\hline 1227 & 38 & 38 & 38 & 38 & 38 & 38 & 37 & 37 & 38 & 38 \\
\hline 1233 & 38 & 38 & 38 & 38 & 37 & 38 & 37 & 37 & 38 & 38 \\
\hline 1239 & 37 & 38 & 38 & 38 & 37 & 38 & 37 & 37 & 38 & 38 \\
\hline 1245 & 37 & 38 & 38 & 38 & 38 & 38 & 37 & 37 & 38 & 38 \\
\hline 1251 & 37 & 37 & 38 & 38 & 38 & 38 & 37 & 37 & 38 & 38 \\
\hline 1256 & 37 & 37 & 38 & 38 & 37 & 38 & 37 & 37 & 38 & 38 \\
\hline
\end{tabular}


OPEN DOOR PRIVACY CURTAIN TEST

THERMOCOUPLES AND DISKS AT SPRINKLER AND DETECTOR LOCATIONS $\cdot{ }^{\circ} \mathrm{C}$

\begin{tabular}{|c|c|c|c|c|c|c|c|c|c|c|}
\hline TIME & $T 14$ & D15 & T16 & D17 & T18 & D19 & $T 20$ & D21 & T22 & D23 \\
\hline 1262 & 37 & 37 & 38 & 38 & 37 & 37 & 37 & 37 & 38 & 38 \\
\hline 1268 & 37 & 37 & 38 & 38 & 37 & 38 & 37 & 37 & 38 & 38 \\
\hline 1274 & 37 & 37 & 38 & 38 & 38 & 38 & 37 & 37 & 38 & 38 \\
\hline 1280 & 37 & 37 & 38 & 38 & 37 & 38 & 37 & 37 & 38 & 38 \\
\hline 1286 & 37 & 37 & 38 & 38 & 38 & 38 & 37 & 37 & 38 & 38 \\
\hline 1291 & 37 & 37 & 38 & 38 & 38 & 38 & 37 & 37 & 38 & 38 \\
\hline 1297 & 37 & 37 & 38 & 38 & 38 & 38 & 37 & 37 & 38 & 38 \\
\hline 1303 & 38 & 37 & 38 & 38 & 37 & 38 & 37 & 37 & 38 & 38 \\
\hline 1309 & 37 & 37 & 38 & 38 & 38 & 38 & 37 & 37 & 38 & 38 \\
\hline 1315 & 37 & 37 & 38 & 38 & 37 & 38 & 37 & 37 & 38 & 38 \\
\hline 1321 & 37 & 37 & 38 & 38 & 37 & 37 & 37 & 37 & 37 & 38 \\
\hline 1327 & 37 & 37 & 38 & 38 & 37 & 37 & 37 & 37 & 37 & 38 \\
\hline 1332 & 37 & 37 & 38 & 38 & 37 & 38 & 37 & 37 & 37 & 38 \\
\hline 1338 & 37 & 37 & 38 & 38 & 37 & 38 & 37 & 37 & 38 & 37 \\
\hline 1344 & 37 & 37 & 38 & 38 & 37 & 38 & 37 & 37 & 37 & 38 \\
\hline 1350 & 37 & 37 & 38 & 38 & 37 & 37 & 37 & 37 & 37 & 37 \\
\hline 1356 & 37 & 37 & 38 & 38 & 37 & 38 & 37 & 37 & 37 & 37 \\
\hline 1362 & 37 & 38 & 38 & 38 & 37 & 37 & 37 & 37 & 37 & 37 \\
\hline 1367 & 37 & 37 & 38 & 38 & 37 & 37 & 37 & 37 & 37 & 37 \\
\hline 1373 & 37 & 37 & 38 & 38 & 37 & 38 & 37 & 37 & 38 & 38 \\
\hline 1379 & 37 & 37 & 38 & 38 & 38 & 38 & 37 & 37 & 37 & 37 \\
\hline 1385 & 37 & 37 & 38 & 38 & 37 & 38 & 37 & 37 & 37 & 37 \\
\hline 1391 & 37 & 37 & 38 & 38 & 37 & 38 & 37 & 37 & 37 & 37 \\
\hline 1397 & 37 & 37 & 38 & 38 & 37 & 38 & 37 & 37 & 37 & 37 \\
\hline 1402 & 37 & 37 & 38 & 38 & 37 & 38 & 37 & 37 & 37 & 37 \\
\hline
\end{tabular}


OPEN DOOR PRIVACY CURTAIN TEST

THERMOCOUPLES AND DISKS AT SPRINKLER AND DETECTOR LOCATIONS - ${ }^{\circ} \mathrm{C}$

\begin{tabular}{|c|c|c|c|c|c|c|c|c|c|c|}
\hline TME & $\mathrm{T} 14$ & D15 & T16 & D17 & $\mathrm{T} 18$ & D19 & $\mathrm{T} 20$ & $\mathrm{D} 21$ & T22 & $\mathrm{D} 23$ \\
\hline 1408 & 37 & 37 & 38 & 38 & 37 & 37 & 37 & 37 & 38 & 37 \\
\hline 1414 & 37 & 37 & 38 & 38 & 38 & 38 & 37 & 37 & 37 & 37 \\
\hline 1420 & 37 & 37 & 38 & 38 & 38 & 38 & 37 & 37 & 38 & 37 \\
\hline 1426 & 37 & 37 & 38 & 38 & 38 & 38 & 37 & 37 & 37 & 37 \\
\hline 1432 & 37 & 37 & 38 & 38 & 38 & 38 & 37 & 37 & 37 & 37 \\
\hline 1437 & 37 & 37 & 38 & 38 & 38 & 38 & 37 & 37 & 37 & 37 \\
\hline 1443 & 37 & 37 & 38 & 38 & 37 & 38 & 37 & 37 & 37 & 37 \\
\hline 1449 & 37 & 37 & 38 & 38 & 38 & 38 & 37 & 37 & 37 & 37 \\
\hline 1455 & 38 & 38 & 38 & 38 & 37 & 38 & 37 & 37 & 37 & 37 \\
\hline 1461 & 37 & 38 & 38 & 38 & 37 & 37 & 37 & 37 & 37 & 37 \\
\hline 1467 & 37 & 37 & 38 & 38 & 37 & 37 & 37 & 37 & 37 & 37 \\
\hline 1473 & 37 & 37 & 38 & 38 & 37 & 37 & 37 & 37 & 37 & 37 \\
\hline 1479 & 37 & 38 & 38 & 38 & 37 & 37 & 37 & 37 & 38 & 37 \\
\hline 1484 & 37 & 37 & 38 & 38 & 37 & 37 & 37 & 37 & 37 & 37 \\
\hline 1490 & 37 & 37 & 38 & 38 & 37 & 37 & 37 & 37 & 38 & 37 \\
\hline 1496 & 37 & 37 & 38 & 38 & 37 & 37 & 37 & 37 & 37 & 37 \\
\hline 1502 & 37 & 37 & 38 & 38 & 37 & 38 & 37 & 37 & 37 & 37 \\
\hline 1508 & 37 & 37 & 38 & 38 & 38 & 38 & 37 & 37 & 37 & 37 \\
\hline 1514 & 37 & 37 & 38 & 38 & 37 & 38 & 37 & 37 & 37 & 37 \\
\hline 1519 & 37 & 38 & 38 & 38 & 37 & 37 & 37 & 37 & 37 & 37 \\
\hline 1525 & 37 & 37 & 38 & 38 & 37 & 37 & 37 & 37 & 37 & 37 \\
\hline 1531 & 37 & 37 & 38 & 38 & 37 & 37 & 37 & 37 & 37 & 37 \\
\hline 1537 & 37 & 37 & 38 & 38 & 37 & 37 & 37 & 37 & 37 & 37 \\
\hline 1543 & 37 & 37 & 38 & 38 & 38 & 38 & 37 & 37 & 37 & 37 \\
\hline 1549 & 38 & 38 & 38 & 38 & 37 & 38 & 37 & 37 & 37 & 37 \\
\hline
\end{tabular}


OPEN DOOR PRIVACY CURTAIN TEST

THERMOCOUPLES AND DISKS AT SPRINKLER AND DETECTOR LOCATIONS - ${ }^{\circ} \mathrm{C}$

\begin{tabular}{llllllllllll}
\hline TME & T14 & D15 & T16 & D17 & T18 & D19 & T20 & D21 & T22 & D23 \\
\hline \hline 1554 & 37 & 38 & 38 & 38 & 38 & 38 & 37 & 37 & 37 & 37 \\
1560 & 37 & 37 & 38 & 38 & 37 & 37 & 37 & 37 & 37 & 37 \\
1566 & 38 & 38 & 38 & 38 & 37 & 38 & 37 & 37 & 37 & 37 \\
1572 & 37 & 37 & 38 & 38 & 37 & 37 & 37 & 37 & 37 & 37 \\
1578 & 37 & 37 & 38 & 38 & 37 & 37 & 37 & 37 & 37 & 37 \\
1584 & 37 & 37 & 38 & 38 & 37 & 37 & 37 & 37 & 38 & 37 \\
1590 & 37 & 37 & 38 & 38 & 37 & 37 & 37 & 37 & 37 & 37 \\
1595 & 37 & 37 & 38 & 38 & 37 & 37 & 36 & 36 & 37 & 37 \\
1601 & 37 & 37 & 38 & 38 & 37 & 37 & 37 & 36 & 37 & 37 \\
1607 & 38 & 38 & 38 & 38 & 37 & 37 & 37 & 36 & 37 & 37 \\
1613 & 37 & 38 & 38 & 38 & 37 & 37 & 37 & 37 & 37 & 37 \\
1619 & 37 & 38 & 38 & 38 & 37 & 37 & 37 & 37 & 37 & 37 \\
1625 & 37 & 37 & 38 & 38 & 37 & 37 & 37 & 36 & 37 & 37
\end{tabular}


OPEN DOOR PRIVACY CURTAIN TEST

THERMOCOUPLES AND DISKS AT SPRINKLER AND DETECTOR LOCATIONS - ${ }^{\circ} \mathrm{C}$

\begin{tabular}{|c|c|c|c|c|c|c|c|c|c|c|}
\hline TIME & $T 24$ & D25 & T26 & D27 & $T 28$ & D29 & PAN A & PAN B & PAN C & PAN D \\
\hline-42 & 25 & 25 & 25 & 25 & 24 & 24 & 24 & 24 & 26 & 24 \\
\hline-34 & 25 & 25 & 25 & 25 & 24 & 24 & 25 & 24 & 26 & 25 \\
\hline-29 & 25 & 25 & 25 & 25 & 24 & 24 & 25 & 24 & 26 & 25 \\
\hline-23 & 25 & 25 & 25 & 25 & 25 & 24 & 25 & 25 & 26 & 25 \\
\hline-17 & 25 & 25 & 25 & 25 & 24 & 24 & 25 & 24 & 26 & 25 \\
\hline-11 & 25 & 25 & 25 & 25 & 24 & 24 & 25 & 24 & 26 & 25 \\
\hline .6 & 25 & 25 & 25 & 25 & 24 & 24 & 24 & 24 & 26 & 25 \\
\hline-0 & 25 & 25 & 25 & 25 & 24 & 24 & 24 & 24 & 26 & 25 \\
\hline 5 & 25 & 25 & 25 & 25 & 24 & 24 & 25 & 24 & 26 & 25 \\
\hline 10 & 25 & 25 & 25 & 25 & 24 & 24 & 26 & 24 & 26 & 25 \\
\hline 16 & 25 & 25 & 25 & 25 & 24 & 24 & 28 & 24 & 26 & 26 \\
\hline 22 & 26 & 25 & 25 & 25 & 25 & 24 & 29 & 25 & 26 & 28 \\
\hline 28 & 27 & 25 & 27 & 25 & 25 & 24 & 30 & 25 & 26 & 28 \\
\hline 33 & 28 & 25 & 28 & 25 & 26 & 24 & 30 & 26 & 26 & 29 \\
\hline 39 & 28 & 25 & 28 & 26 & 26 & 24 & 31 & 26 & 26 & 30 \\
\hline 45 & 29 & 25 & 29 & 26 & 27 & 24 & 32 & 27 & 26 & 30 \\
\hline 50 & 30 & 26 & 30 & 27 & 28 & 25 & 33 & 28 & 26 & 31 \\
\hline 56 & 30 & 26 & 30 & 27 & 28 & 25 & 33 & 28 & 26 & 32 \\
\hline$\sigma 2$ & 31 & 26 & 31 & 27 & 28 & 25 & 35 & 28 & 27 & 33 \\
\hline 68 & 31 & 26 & 31 & 28 & 30 & 25 & 37 & 30 & 27 & 33 \\
\hline 73 & 32 & 27 & 32 & 28 & 31 & 25 & 38 & 31 & 28 & 34 \\
\hline 79 & 32 & 27 & 32 & 29 & 31 & 26 & 38 & 31 & 28 & 35 \\
\hline 85 & 33 & 27 & 33 & 29 & 32 & 26 & 38 & 32 & 28 & 35 \\
\hline 91 & 34 & 27 & 34 & 30 & 33 & 26 & 39 & 33 & 29 & 35 \\
\hline 97 & 34 & 28 & 35 & 30 & 33 & 27 & $40^{\circ}$ & 33 & 29 & 37 \\
\hline
\end{tabular}


OPEN DOOR PRIVACY CURTAIN TEST

THERMOCOUPLES AND DISKS AT SPRINKLER AND DETECTOR LOCATIONS - ${ }^{\circ} \mathrm{C}$

\begin{tabular}{|c|c|c|c|c|c|c|c|c|c|c|}
\hline TTME & T24 & $\mathrm{D} 25$ & T26 & $\mathrm{D} 27$ & $T 28$ & D29 & PAN A & PAN B & PAN C & PAN D \\
\hline 102 & 34 & 28 & 35 & 31 & 33 & 27 & 40 & 33 & 29 & 36 \\
\hline 108 & 35 & 28 & 35 & 31 & 33 & 27 & 41 & 33 & 29 & 37 \\
\hline 114 & 35 & 29 & 36 & 32 & 35 & 28 & 41 & 35 & 29 & 38 \\
\hline 119 & 38 & 29 & 36 & 32 & 35 & 28 & 42 & 35 & 30 & 39 \\
\hline 125 & 37 & 29 & 38 & 33 & 35 & 28 & 43 & 35 & 30 & 39 \\
\hline 131 & 37 & 29 & 38 & 33 & 35 & 29 & 44 & 35 & 30 & 39 \\
\hline 137 & 38 & 30 & 38 & 34 & 36 & 29 & 44 & 36 & 30 & 41 \\
\hline 142 & 39 & 30 & 38 & 34 & 37 & 29 & 45 & 37 & 31 & 41 \\
\hline 148 & 38 & 30 & 39 & 35 & 38 & 30 & 47 & 38 & 31 & 42 \\
\hline 954 & 39 & 31 & 39 & 35 & 38 & 30 & 47 & 38 & 31 & 43 \\
\hline 160 & 40 & 31 & 40 & 36 & 39 & 31 & 48 & 39 & 33 & 43 \\
\hline 165 & 40 & 31 & 41 & 36 & 40 & 39 & 48 & 40 & 33 & 44 \\
\hline 171 & 41 & 32 & 42 & 37 & 40 & 32 & 48 & 40 & 33 & 44 \\
\hline 177 & 41 & 32 & 42 & 37 & 41 & 32 & 48 & 41 & 34 & 46 \\
\hline 182 & 42 & 32 & 42 & 38 & 41 & 32 & 49 & 41 & 34 & 46 \\
\hline 188 & 42 & 33 & 42 & 38 & 42 & 33 & 51 & 42 & 34 & 46 \\
\hline 194 & 43 & 34 & 44 & 39 & 42 & 33 & 51 & 42 & 34 & 46 \\
\hline 200 & 44 & 34 & 45 & 39 & 43 & 34 & 51 & 43 & 35 & 47 \\
\hline 205 & 44 & 34 & 46 & 40 & 43 & 34 & 52 & 43 & 35 & 48 \\
\hline 211 & 44 & 35 & 46 & 40 & 44 & 34 & 53 & 44 & 35 & 48 \\
\hline 217 & 45 & 35 & 47 & 41 & 44 & 35 & 54 & 44 & 35 & 49 \\
\hline 223 & 45 & 35 & 47 & 42 & 44 & 35 & 55 & 44 & 35 & 51 \\
\hline 228 & 47 & 36 & 48 & 42 & 44 & 36 & 55 & 44 & 36 & 51 \\
\hline 234 & 48 & 36 & 48 & 43 & 45 & 36 & 58 & 45 & 37 & 52 \\
\hline 240 & 48 & 37 & 50 & 44 & 46 & 36 & 57 & 46 & 37 & 53 \\
\hline
\end{tabular}


THERMOCOUPLES AND DISKS AT SPRINKLER AND DETECTOR LOCATIONS - ${ }^{\circ} \mathrm{C}$

\begin{tabular}{|c|c|c|c|c|c|c|c|c|c|c|}
\hline TME & T24 & D25 & T26 & $\mathrm{D} 27$ & T28 & D29 & PAN A & PAN B & PAN C & PAN D \\
\hline 246 & 51 & 37 & 52 & 44 & 46 & 37 & 59 & 46 & 37 & 53 \\
\hline 251 & 51 & 38 & 52 & 45 & 47 & 37 & 59 & 47 & 38 & 52 \\
\hline 257 & 51 & 38 & 54 & 46 & 49 & 38 & 59 & 49 & 39 & 56 \\
\hline 263 & 52 & 39 & 53 & 46 & 49 & 38 & 61 & 49 & 38 & 57 \\
\hline 268 & 55 & 39 & 52 & 47 & 49 & 39 & 61 & 49 & 39 & 56 \\
\hline 274 & 53 & 40 & 55 & 48 & 50 & 39 & 62 & 50 & 40 & 58 \\
\hline 280 & 53 & 40 & 55 & 48 & 51 & 40 & 64 & 51 & 41 & 59 \\
\hline 286 & 54 & 41 & 56 & 49 & 51 & 40 & 65 & 51 & 41 & 59 \\
\hline 291 & 55 & 41 & 57 & 50 & 53 & 41 & 65 & 53 & 41 & 60 \\
\hline 297 & 57 & 42 & 59 & 50 & 53 & 41 & 66 & 53 & 41 & 59 \\
\hline 303 & 56 & 42 & 58 & 51 & 53 & 42 & 69 & 53 & 42 & 61 \\
\hline 308 & 57 & 43 & 58 & 52 & 54 & 42 & 68 & 54 & 42 & 61 \\
\hline 314 & 57 & 43 & 60 & 52 & 54 & 43 & 70 & 54 & 43 & $\sigma 2$ \\
\hline 320 & 58 & 44 & 60 & 53 & 55 & 43 & 70 & 55 & 43 & 64 \\
\hline 326 & 59 & 44 & 63 & 54 & 57 & 44 & 72 & 57 & 44 & 64 \\
\hline 331 & 60 & 45 & 63 & 55 & 57 & 44 & 71 & 57 & 44 & 65 \\
\hline 337 & 62 & 45 & 63 & 55 & 58 & 45 & 74 & 58 & 45 & 66 \\
\hline 343 & $\curvearrowleft 2$ & 46 & 63 & 56 & 60 & 46 & 76 & 60 & 47 & 68 \\
\hline 349 & 63 & 46 & 64 & 56 & 60 & 46 & 76 & 60 & 46 & 67 \\
\hline 354 & 64 & 47 & 66 & 57 & 62 & 47 & 73 & 62 & 47 & 67 \\
\hline 360 & 66 & 47 & 66 & 58 & 60 & 48 & 74 & 60 & 47 & 69 \\
\hline 366 & 67 & 48 & 67 & 59 & 61 & 48 & $\pi$ & 61 & 49 & 71 \\
\hline 372 & 66 & 49 & 68 & 59 & 64 & 49 & $\pi$ & 64 & 48 & 72 \\
\hline 377 & 68 & 49 & 69 & 60 & 62 & 49 & 78 & 62 & 49 & 73 \\
\hline 383 & 67 & 50 & 69 & 61 & 64 & 50 & 79 & 64 & 49 & 72 \\
\hline
\end{tabular}


OPEN DOOR PRIVACY CURTAIN TEST

THERMOCOUPLES AND DISKS AT SPRINKLER AND DETECTOR LOCATIONS $\cdot{ }^{\circ} \mathrm{C}$

\begin{tabular}{|c|c|c|c|c|c|c|c|c|c|c|}
\hline TMME & $T 24$ & D25 & T26 & D27 & T28 & D29 & PAN A & PAN B & PAN C & PAN D \\
\hline 389 & 67 & 50 & 70 & 62 & 65 & 50 & 80 & 65 & 49 & 74 \\
\hline 395 & 67 & 51 & 70 & 62 & 65 & 51 & 83 & 65 & 51 & 74 \\
\hline 400 & 67 & 51 & 70 & 63 & 66 & 52 & 83 & 66 & 50 & 74 \\
\hline 406 & 67 & 51 & 71 & 63 & 66 & 52 & 82 & 66 & 50 & 75 \\
\hline 412 & 68 & 52 & 72 & 64 & 67 & 53 & 82 & 67 & 51 & 78 \\
\hline 417 & 69 & 52 & 71 & 64 & 67 & 53 & 83 & 67 & 53 & 76 \\
\hline 423 & 69 & 52 & 72 & 64 & 66 & 54 & 81 & 66 & 53 & 75 \\
\hline 429 & 67 & 53 & 71 & 65 & 67 & 54 & 77 & 67 & 52 & 74 \\
\hline 435 & 66 & 53 & 71 & 65 & 66 & 54 & 80 & 66 & 52 & 75 \\
\hline 440 & 67 & 53 & 70 & 65 & 65 & 54 & 79 & 65 & 52 & $\pi$ \\
\hline 446 & $\omega$ & 54 & 69 & 65 & 65 & 55 & 79 & 65 & 51 & 74 \\
\hline 452 & 68 & 54 & 70 & 65 & 66 & 55 & 81 & 66 & 51 & 75 \\
\hline 458 & 69 & 54 & 70 & 65 & 65 & 55 & 79 & 65 & 52 & 75 \\
\hline 463 & 70 & 54 & 71 & 65 & 65 & 55 & 80 & 65 & 52 & 75 \\
\hline 469 & 69 & 55 & 72 & 65 & 68 & 56 & 83 & 68 & 52 & 76 \\
\hline 475 & 68 & 55 & 72 & 65 & 67 & 56 & 86 & 67 & 52 & 78 \\
\hline 480 & 70 & 55 & 74 & 66 & 68 & 56 & 85 & 68 & 54 & $\pi$ \\
\hline 486 & 71 & 55 & 75 & 67 & 69 & 57 & 88 & 69 & 53 & 78 \\
\hline 492 & 70 & 56 & 72 & 67 & 69 & 57 & 86 & 69 & 54 & 79 \\
\hline 498 & 72 & 56 & 74 & 67 & 70 & 57 & 87 & 70 & 56 & 80 \\
\hline 504 & 74 & 57 & 76 & 68 & 69 & 58 & 90 & 69 & 55 & 80 \\
\hline 509 & 75 & 57 & 78 & 68 & 72 & 58 & 90 & 72 & 54 & 82 \\
\hline 515 & 72 & 57 & 80 & 69 & 71 & 58 & 92 & 71 & 57 & 83 \\
\hline 521 & 72 & 58 & 79 & 70 & 72 & 59 & 91 & 72 & 57 & 86 \\
\hline 526 & 76 & 58 & 81 & 71 & 74 & 59 & 94 & 74 & 57 & 86 \\
\hline
\end{tabular}


OPEN DOOR PAIVACY CURTAIN TEST

THERMOCOUPLES AND DISKS AT SPRINKLER AND DETECTOR LOCATIONS - ${ }^{\circ} \mathrm{C}$

\begin{tabular}{|c|c|c|c|c|c|c|c|c|c|c|}
\hline TIME & T24 & D25 & T26 & 027 & T28 & D29 & PAN A & PAN B & PAN C & PAN D \\
\hline 532 & $\pi$ & 59 & 83 & 72 & 74 & 60 & 94 & 74 & 59 & 85 \\
\hline 538 & $\pi$ & 59 & 82 & 72 & 74 & 60 & 96 & 74 & 58 & 87 \\
\hline 544 & 81 & 60 & 80 & 73 & 76 & 61 & 96 & 76 & 59 & 91 \\
\hline 549 & 79 & 61 & 82 & 73 & $\pi$ & 62 & 99 & $\pi$ & 60 & 91 \\
\hline 555 & 81 & 61 & 84 & 74 & 78 & 62 & 100 & 78 & 60 & 93 \\
\hline 561 & 82 & 62 & 89 & 76 & $\pi$ & 63 & 100 & 77 & 61 & 92 \\
\hline 567 & 83 & 63 & 88 & 77 & 78 & 63 & 102 & 78 & 63 & 93 \\
\hline 573 & 83 & 63 & 90 & 78 & 80 & 64 & 104 & 80 & 61 & 94 \\
\hline 579 & 82 & 64 & 88 & 79 & 81 & 64 & 103 & 81 & 62 & 99 \\
\hline 584 & 86 & 64 & 89 & 79 & 83 & 65 & 105 & 83 & 63 & 97 \\
\hline 590 & 84 & 65 & 91 & 80 & 82 & 66 & 103 & 82 & 64 & 98 \\
\hline 596 & 86 & 66 & 89 & 81 & 82 & 66 & 104 & 82 & 65 & 98 \\
\hline 602 & 85 & 66 & 93 & 82 & 82 & 67 & 105 & 82 & 65 & 101 \\
\hline 608 & 88 & 67 & 91 & 82 & 83 & 67 & 110 & 83 & 65 & 102 \\
\hline 614 & 89 & 68 & 95 & 83 & 83 & 68 & 109 & 83 & 67 & 104 \\
\hline 619 & 93 & 69 & 95 & 84 & 84 & 68 & 109 & 84 & 67 & 103 \\
\hline 625 & 88 & 69 & 96 & 85 & 86 & 69 & 111 & 86 & 67 & 103 \\
\hline 631 & 92 & 70 & 97 & 86 & 86 & 70 & 113 & 86 & 67 & 106 \\
\hline 637 & 95 & $\approx 2$ & 98 & 86 & 86 & 70 & 117 & 86 & 68 & 106 \\
\hline 643 & 96 & 61 & 98 & 87 & 88 & 71 & 115 & 88 & 68 & 104 \\
\hline 649 & 99 & 63 & 103 & 88 & 89 & 72 & 115 & 89 & 70 & 105 \\
\hline 655 & 98 & 65 & 101 & 89 & 92 & 73 & 117 & 92 & 70 & 109 \\
\hline 660 & 98 & 67 & 103 & 90 & 90 & 73 & 122 & 90 & 72 & 110 \\
\hline 666 & 99 & 68 & 100 & 91 & 92 & 74 & 120 & 92 & 71 & 109 \\
\hline 672 & 100 & 70 & 103 & 91 & 94 & 75 & 124 & 94 & 73 & 108 \\
\hline
\end{tabular}


OPEN DOOR PRIVACY CURTAIN TEST

THERMOCOUPLES AND DISKS AT SPRINKLER AND DETECTOR LOCATIONS - ${ }^{\circ} \mathrm{C}$

\begin{tabular}{|c|c|c|c|c|c|c|c|c|c|c|}
\hline TIME & T24 & $D 25$ & T26 & $\mathrm{D} 27$ & T28 & D29 & PAN A & PAN B & PAN C & PAN D \\
\hline 678 & 102 & 71 & 107 & 92 & 92 & 76 & 122 & 92 & 73 & 110 \\
\hline 684 & 101 & 73 & 106 & 93 & 93 & 76 & 121 & 93 & 74 & 111 \\
\hline 690 & 102 & 74 & 106 & 94 & 95 & $\pi$ & 125 & 95 & 75 & 111 \\
\hline 695 & 105 & 75 & 108 & 95 & 97 & 78 & 122 & 97 & 74 & 109 \\
\hline 701 & 103 & 76 & 107 & 96 & 93 & 78 & 122 & 93 & 74 & 113 \\
\hline 707 & 104 & 78 & 110 & 96 & 94 & 79 & 127 & 94 & 72 & 111 \\
\hline 713 & 105 & 79 & 112 & 98 & 98 & 80 & 128 & 98 & 74 & 116 \\
\hline 719 & 106 & 79 & 111 & 98 & 95 & 80 & 126 & 95 & 78 & 116 \\
\hline 725 & 104 & 80 & 108 & 98 & 96 & 80 & 124 & 96 & 76 & 115 \\
\hline 730 & 108 & 81 & 107 & 98 & 98 & 81 & 127 & 98 & 77 & 116 \\
\hline 736 & 105 & 81 & 109 & 99 & 99 & 81 & 132 & 99 & $\pi$ & 116 \\
\hline 742 & 107 & 82 & 111 & 99 & 96 & 82 & 126 & 96 & $\pi$ & 115 \\
\hline 748 & 106 & 82 & 112 & 100 & 97 & 82 & 130 & 97 & 78 & 117 \\
\hline 754 & 111 & 83 & 108 & 100 & 97 & 83 & 124 & 97 & 77 & 117 \\
\hline 759 & 106 & 83 & 111 & 100 & 98 & 83 & 128 & 98 & 79 & 117 \\
\hline 765 & 110 & 84 & 111 & 100 & 100 & 84 & 130 & 100 & 77 & 116 \\
\hline 77 & 111 & 84 & 113 & 101 & 98 & 84 & 133 & 98 & 79 & 120 \\
\hline 777 & 112 & 85 & 115 & 102 & 97 & 85 & 127 & 97 & 79 & 120 \\
\hline 783 & 111 & 86 & 112 & 102 & 99 & 85 & 136 & 99 & 81 & 119 \\
\hline 789 & 114 & 86 & 113 & 102 & 102 & 86 & 134 & 102 & 82 & 123 \\
\hline 794 & 116 & 88 & 117 & 103 & 103 & 87 & 130 & 103 & 83 & 120 \\
\hline 800 & 112 & 88 & 117 & 104 & 102 & 87 & 134 & 102 & 80 & 121 \\
\hline 806 & 115 & 89 & 116 & 104 & 103 & 87 & 135 & 103 & 79 & 125 \\
\hline 812 & 118 & 90 & 116 & 105 & 101 & 88 & 132 & 101 & 83 & 125 \\
\hline 818 & 52 & 79 & 92 & 97 & 0 & - & 40 & 32 & 84 & 71 \\
\hline
\end{tabular}


OPEN DOOR PRIVACY CURTAIN TEST

THERMOCOUPLES AND DISKS AT SPRINKLER AND DETECTOR LOCATIONS - ${ }^{\circ} \mathrm{C}$

\begin{tabular}{|c|c|c|c|c|c|c|c|c|c|c|}
\hline TME & $T 24$ & $\mathrm{D} 25$ & T26 & $\mathrm{D} 27$ & T28 & D29 & PAN A & PAN B & PAN C & PAN D \\
\hline 823 & 54 & 71 & 40 & 59 & 0 & - & 42 & 34 & 86 & 93 \\
\hline 829 & 66 & 67 & 39 & 46 & 0 & - & 38 & 33 & 78 & 92 \\
\hline 835 & 49 & 51 & 38 & 40 & 0 & - & 36 & 33 & 70 & 91 \\
\hline 841 & 45 & 44 & 37 & 37 & 0 & - & 35 & 32 & 63 & 86 \\
\hline 847 & 43 & 42 & 36 & 37 & 32 & --- & 34 & 32 & 56 & 86 \\
\hline 853 & 39 & 38 & 36 & 36 & 33 & - & 34 & 33 & 52 & 72 \\
\hline 858 & 37 & 37 & 36 & 36 & 32 & - & 36 & 32 & 49 & 66 \\
\hline 864 & 36 & 36 & 35 & 35 & 32 & - & 34 & 32 & 48 & 67 \\
\hline 870 & 34 & 35 & 35 & 35 & 31 & - & 33 & 31 & 45 & 55 \\
\hline 876 & 35 & 35 & 34 & 35 & 30 & - & 33 & 30 & 43 & 49 \\
\hline 882 & 34 & 34 & 34 & 34 & 30 & --- & 33 & 30 & 43 & 55 \\
\hline 888 & 33 & 35 & 33 & 34 & 30 & - & 32 & 30 & 42 & 52 \\
\hline 894 & 33 & 34 & 34 & 34 & 29 & - & 32 & 29 & 42 & 55 \\
\hline 899 & 33 & 33 & 33 & 34 & 29 & - & 31 & 29 & 42 & 45 \\
\hline 905 & 32 & 33 & 33 & 33 & 29 & - & 32 & 29 & 42 & 48 \\
\hline 911 & 33 & 32 & 31 & 33 & 29 & - & 31 & 29 & 42 & 48 \\
\hline 917 & 31 & 32 & 32 & 32 & 29 & - & 31 & 29 & 40 & 46 \\
\hline 923 & 32 & 32 & 31 & 32 & 30 & - & 31 & 30 & 40 & 46 \\
\hline 929 & 32 & 32 & 32 & 32 & 30 & - & 31 & 30 & 39 & 48 \\
\hline 935 & 31 & 31 & 32 & 32 & 30 & - & 31 & 30 & 39 & 49 \\
\hline 940 & 32 & 31 & 32 & 32 & 30 & - & 31 & 30 & 38 & 46 \\
\hline 946 & 32 & 32 & 32 & 32 & 30 & - & 31 & 30 & 37 & 39 \\
\hline 952 & 31 & 31 & 32 & 32 & 30 & - & 31 & 30 & 37 & 40 \\
\hline 958 & 31 & 31 & 32 & 32 & 29 & - & 31 & 29 & 38 & 46 \\
\hline 964 & 31 & 31 & 32 & 32 & 29 & - & 30 & 29 & 38 & 39 \\
\hline
\end{tabular}




\section{THERMOCOUPLES AND DISKS AT SPRINKLER AND DETECTOR LOCATIONS - ${ }^{\circ} \mathrm{C}$}

\begin{tabular}{|c|c|c|c|c|c|c|c|c|c|c|}
\hline TMME & T24 & D25 & T26 & D27 & T28 & D29 & PAN A & PAN B & PAN C & PAN D \\
\hline 970 & 30 & 31 & 32 & 32 & 29 & - & 30 & 29 & 37 & 42 \\
\hline 976 & 32 & 31 & 31 & 32 & 29 & - & 30 & 29 & 35 & 40 \\
\hline 982 & 30 & 31 & 31 & 32 & 29 & - & 30 & 29 & 36 & 30 \\
\hline 987 & 33 & 31 & 31 & 31 & 30 & - & 31 & 30 & 36 & 37 \\
\hline 993 & 31 & 31 & 31 & 31 & 31 & - & 31 & 31 & 36 & 41 \\
\hline 999 & 32 & 31 & 31 & 31 & 32 & - & 31 & 32 & 36 & 42 \\
\hline 1005 & 31 & 31 & 31 & 31 & 34 & - & 32 & 34 & 35 & 40 \\
\hline 1011 & 32 & 32 & 32 & 31 & 35 & - & 33 & 35 & 33 & 38 \\
\hline 1017 & 33 & 32 & 32 & 32 & 36 & - & 34 & 36 & 33 & 36 \\
\hline 1023 & 33 & 33 & 33 & 32 & 37 & - & 34 & 37 & 34 & 35 \\
\hline 1028 & 34 & 33 & 33 & 32 & 38 & - & 35 & 38 & 34 & 36 \\
\hline 1034 & 34 & 34 & 33 & 33 & 38 & - & 35 & 38 & 34 & 36 \\
\hline 1040 & 34 & 34 & 34 & 33 & 39 & - & 36 & 39 & 35 & 36 \\
\hline 1046 & 35 & 34 & 34 & 33 & 0 & - & 36 & 39 & 35 & 35 \\
\hline 1052 & 35 & 35 & 34 & 34 & 0 & - & 36 & 39 & 34 & 35 \\
\hline 1058 & 35 & 35 & 34 & 34 & 0 & - & 36 & 38 & 34 & 36 \\
\hline 1064 & 35 & 35 & 35 & 34 & 0 & - & 36 & 39 & 33 & 36 \\
\hline 1069 & 35 & 35 & 35 & 34 & 0 & - & 36 & 39 & 33 & 36 \\
\hline 1075 & 35 & 35 & 35 & 35 & 0 & - & 36 & 39 & 33 & 36 \\
\hline 1081 & 36 & 35 & 35 & 35 & 0 & - & 37 & 39 & 34 & 36 \\
\hline 1087 & 36 & 35 & 35 & 35 & 0 & - & 37 & 39 & 34 & 38 \\
\hline 1093 & 36 & 36 & 35 & 35 & 0 & - & 37 & 39 & 35 & 37 \\
\hline 1099 & 36 & 36 & 35 & 35 & 0 & - & 37 & 39 & 34 & 37 \\
\hline 1104 & 35 & 36 & 35 & 35 & 0 & - & 37 & 39 & 34 & 36 \\
\hline 1110 & 36 & 36 & 35 & 35 & 0 & - & 37 & 39 & 33 & 36 \\
\hline
\end{tabular}


THERMOCOUPLES AND DISKS AT SPRINKLER AND DETECTOR LOCATIONS - ${ }^{\circ} \mathrm{C}$

\begin{tabular}{|c|c|c|c|c|c|c|c|c|c|c|}
\hline TIME & T24 & D25 & T26 & $\mathrm{D} 27$ & T28 & D29 & PAN A & PAN B & PAN C & PAN D \\
\hline 1116 & 35 & 36 & 35 & 35 & 0 & $\cdots$ & 37 & 39 & 33 & 36 \\
\hline 1122 & 36 & 36 & 35 & 35 & 0 & - & 37 & 39 & 33 & 36 \\
\hline 1128 & 36 & 36 & 36 & 35 & 0 & -- & 37 & 39 & 33 & 36 \\
\hline 1134 & 36 & 36 & 35 & 35 & 0 & $-\infty$ & 37 & 40 & 33 & 37 \\
\hline 1139 & 36 & 36 & 35 & 35 & 0 & --. & 37 & 40 & 33 & 37 \\
\hline 1145 & 36 & 36 & 34 & 35 & 0 & - & 37 & 40 & 33 & 37 \\
\hline 1151 & 36 & 36 & 35 & 35 & 0 & -- & 37 & 40 & 33 & 37 \\
\hline 1157 & 36 & 36 & 35 & 35 & 0 & - & 37 & 40 & 33 & 37 \\
\hline 1163 & 36 & 36 & 36 & 35 & 0 & - & 37 & 40 & 33 & 37 \\
\hline 1169 & 36 & 36 & 36 & 36 & 0 & - & 38 & 40 & 33 & 36 \\
\hline 1175 & 36 & 36 & 36 & 36 & 0 & - & 38 & 40 & 33 & 36 \\
\hline 1180 & 36 & 36 & 36 & 36 & 0 & - & 37 & 40 & 33 & 37 \\
\hline 1186 & 36 & 36 & 36 & 36 & 0 & --. & 38 & 40 & 33 & 37 \\
\hline 1192 & 37 & 36 & 36 & 36 & 0 & - & 38 & 40 & 33 & 37 \\
\hline 1198 & 36 & 36 & 36 & 36 & 0 & - & 38 & 40 & 32 & 37 \\
\hline 1204 & 37 & 36 & 36 & 36 & 0 & - & 38 & 40 & 32 & 37 \\
\hline 1210 & 36 & 36 & 36 & 36 & 0 & - & 38 & 39 & 34 & 37 \\
\hline 1215 & 37 & 36 & 36 & 36 & 0 & - & 38 & 40 & 33 & 37 \\
\hline 1221 & 36 & 37 & 36 & 36 & 0 & -- & 38 & 40 & 33 & 37 \\
\hline 1227 & 36 & 36 & 36 & 36 & 0 & - & 38 & 40 & 33 & 37 \\
\hline 1233 & 37 & 37 & 36 & 36 & 0 & - & 38 & 40 & 33 & 37 \\
\hline 1239 & 36 & 36 & 36 & 36 & 0 & - & 38 & 40 & 33 & 37 \\
\hline 1245 & 36 & 36 & 36 & 36 & 0 & - & 38 & 40 & 33 & 37 \\
\hline 1251 & 37 & 37 & 36 & 36 & 0 & - & 38 & 40 & 33 & 37 \\
\hline 1256 & 36 & 37 & 35 & 36 & 0 & - & 38 & 40 & 33 & 37 \\
\hline
\end{tabular}


THERMOCOUPLES AND DISKS AT SPRINKLER AND DETECTOR LOCATIONS - ${ }^{\circ} \mathrm{C}$

\begin{tabular}{|c|c|c|c|c|c|c|c|c|c|c|}
\hline TME & T24 & D25 & T26 & D27 & T28 & D29 & PAN A & PAN B & PAN C & PAN D \\
\hline 1262 & 36 & 36 & 36 & 36 & 0 & - & 38 & 40 & 33 & 37 \\
\hline 1268 & 37 & 37 & 36 & 36 & 0 & - & 38 & 40 & 33 & 37 \\
\hline 1274 & 36 & 37 & 36 & 36 & 0 & - & 38 & 40 & 32 & 37 \\
\hline 1280 & 36 & 36 & 36 & 36 & 0 & - & 38 & 40 & 33 & 37 \\
\hline 1286 & 37 & 37 & 36 & 36 & 0 & - & 38 & 40 & 33 & 37 \\
\hline 1291 & 36 & 37 & 36 & 36 & 0 & 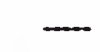 & 38 & 40 & 33 & 37 \\
\hline 1297 & 37 & 37 & 36 & 36 & 0 & - & 38 & 40 & 34 & 37 \\
\hline 1303 & 37 & 37 & 36 & 36 & 0 & - & 38 & 40 & 33 & 37 \\
\hline 1309 & 36 & 36 & 36 & 36 & 0 & - & 38 & 40 & 33 & 37 \\
\hline 1315 & 37 & 36 & 36 & 36 & 0 & - & 38 & 40 & 33 & 37 \\
\hline 1321 & 36 & 36 & 35 & 36 & 0 & - & 38 & 40 & 32 & 37 \\
\hline 1327 & 37 & 37 & 36 & 36 & 0 & - & 38 & 40 & 33 & 37 \\
\hline 1332 & 36 & 36 & 35 & 36 & 0 & $\ldots$ & 37 & 39 & 32 & 37 \\
\hline 1338 & 37 & 37 & 36 & 36 & 0 & - & 38 & 40 & 32 & 37 \\
\hline 1344 & 36 & 37 & 36 & 36 & 0 & - & 38 & 40 & 33 & 37 \\
\hline 1350 & 36 & 36 & 36 & 36 & 0 & - & 38 & 40 & 32 & 37 \\
\hline 1356 & 37 & 37 & 36 & 36 & 0 & - & 38 & 40 & 33 & 37 \\
\hline 1362 & 36 & 36 & 35 & 36 & 0 & - & 38 & 39 & 32 & 37 \\
\hline 1367 & 36 & 36 & 35 & 35 & 0 & - & 38 & 40 & 32 & 37 \\
\hline 1373 & 36 & 36 & 35 & 35 & 0 & - & 38 & 39 & 32 & 37 \\
\hline 1379 & 36 & 36 & 35 & 35 & 0 & - & 38 & 39 & 32 & 37 \\
\hline 1385 & 37 & 36 & 36 & 36 & 0 & - & 38 & 40 & 32 & 37 \\
\hline 1391 & 36 & 36 & 35 & 35 & 0 & - & 38 & 40 & 32 & 37 \\
\hline 1397 & 37 & 36 & 35 & 35 & 0 & - & 38 & 39 & 32 & 37 \\
\hline 1402 & 36 & 36 & 35 & 35 & 0 & 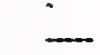 & 38 & 40 & 32 & 37 \\
\hline
\end{tabular}


THERMOCOUPLES AND DISKS AT SPRINKLER AND DETECTOR LOCATIONS - ${ }^{\circ} \mathrm{C}$

\begin{tabular}{|c|c|c|c|c|c|c|c|c|c|c|}
\hline TIME & $T 24$ & $D 25$ & T26 & D27 & T28 & D29 & PAN A & PAN B & PAN C & PAN D \\
\hline 1408 & 36 & 36 & 35 & 35 & 0 & - & 38 & 39 & 32 & 37 \\
\hline 1414 & 37 & 36 & 36 & 35 & 0 & - & 38 & 39 & 32 & 37 \\
\hline 1420 & 36 & 36 & 35 & 35 & 0 & - & 38 & 39 & 33 & 37 \\
\hline 1426 & 36 & 36 & 36 & 35 & 0 & - & 38 & 39 & 33 & 37 \\
\hline 1432 & 36 & 36 & 35 & 36 & 0 & - & 37 & 39 & 33 & 37 \\
\hline 1437 & 36 & 36 & 35 & 35 & 0 & $\cdots$ & 38 & 39 & 32 & 37 \\
\hline 1443 & 37 & 37 & 36 & 36 & 0 & --. & 38 & 40 & 32 & 37 \\
\hline 1449 & 36 & 36 & 36 & 36 & 0 & - & 37 & 38 & 32 & 37 \\
\hline 1455 & 37 & 37 & 36 & 36 & 0 & - & 38 & 39 & 32 & 37 \\
\hline 1461 & 36 & 36 & 36 & 36 & 0 & - & 38 & 39 & 33 & 37 \\
\hline 1467 & 36 & 36 & 36 & 36 & 0 & & 38 & 39 & 32 & 37 \\
\hline 1473 & 37 & 36 & 36 & 36 & 0 & - & 38 & 40 & 34 & 37 \\
\hline 1479 & 36 & 36 & 35 & 36 & 0 & - & 38 & 39 & 33 & 37 \\
\hline 1484 & 36 & 36 & 35 & 36 & 0 & - & 38 & 39 & 32 & 37 \\
\hline 1490 & 36 & 36 & 36 & 36 & 0 & - & 38 & 39 & 32 & 37 \\
\hline 1496 & 36 & 36 & 35 & 36 & 0 & - & 38 & 39 & 32 & 37 \\
\hline 1502 & 36 & 36 & 36 & 36 & 0 & - & 38 & 39 & 32 & 37 \\
\hline 1508 & 36 & 36 & 35 & 35 & 0 & - & 38 & 39 & 32 & 37 \\
\hline 1514 & 36 & 36 & 36 & 35 & 0 & - & 38 & 39 & 32 & 37 \\
\hline 1519 & 36 & 36 & 35 & 36 & 0 & - & 38 & 39 & 33 & 37 \\
\hline 1525 & 36 & 36 & 36 & 36 & 0 & - & 38 & 39 & 32 & 37 \\
\hline 1531 & 37 & 36 & 36 & 36 & 0 & - & 37 & 39 & 32 & 37 \\
\hline 1537 & 36 & 36 & 36 & 36 & 0 & - & 37 & 39 & 32 & 37 \\
\hline 1543 & 36 & 36 & 36 & 36 & 0 & - & 38 & 39 & 32 & 37 \\
\hline 1549 & 37 & 37 & 36 & 36 & 0 & - & 38 & 39 & 32 & 37 \\
\hline
\end{tabular}


OPEN DOOR PRIVACY CURTAIN TEST

THERMOCOUPLES AND DISKS AT SPRINKLER AND DETECTOR LOCATIONS - ${ }^{\circ} \mathrm{C}$

\begin{tabular}{lllllllllll}
\hline TME & T24 & D25 & T26 & D27 & T28 & D29 & PAN A & PAN B & PAN C & PAN D \\
\hline \hline 1554 & 36 & 37 & 36 & 36 & 0 & - & 38 & 39 & 32 & 37 \\
1560 & 37 & 37 & 36 & 36 & 0 & - & 37 & 39 & 32 & 37 \\
1566 & 37 & 37 & 36 & 36 & 0 & - & 37 & 39 & 32 & 37 \\
1572 & 36 & 36 & 36 & 36 & 0 & - & 37 & 39 & 32 & 37 \\
1578 & 36 & 36 & 35 & 36 & 0 & - & 38 & 39 & 33 & 37 \\
1584 & 36 & 36 & 36 & 36 & 0 & - & 37 & 39 & 33 & 37 \\
1590 & 36 & 36 & 35 & 36 & 0 & - & 37 & 39 & 32 & 37 \\
1595 & 36 & 36 & 36 & 36 & 0 & - & 38 & 39 & 32 & 36 \\
1601 & 36 & 36 & 35 & 36 & 0 & - & 37 & 39 & 32 & 37 \\
1607 & 36 & 36 & 35 & 35 & 0 & - & 38 & 39 & 31 & 37 \\
1613 & 36 & 36 & 36 & 35 & 0 & -36 & 37 & 39 & 31 & 37 \\
1619 & 36 & 36 & 36 & 36 & 0 & -36 & 37 & 39 & 31 & 37 \\
1625 & 36 & 36 & 36 & 36 & 0 & -37 & 39 & 32 & 37
\end{tabular}


THERMOCOUPLES AND DISKS AT SPRINKLER AND DETECTOR LOCATIONS $-{ }^{\circ} \mathrm{C}$

\begin{tabular}{|c|c|c|c|c|c|c|c|c|c|c|}
\hline TIME & $\mathrm{T} 14$ & D15 & T16 & D17 & T18 & D19 & T20 & $\mathrm{D} 21$ & T22 & D23 \\
\hline-53 & 29 & 27 & 29 & 26 & 29 & 27 & 28 & 27 & 28 & 27 \\
\hline-49 & 27 & 29 & 26 & 30 & 27 & 29 & 27 & 29 & 27 & 29 \\
\hline-46 & 27 & 29 & 26 & 30 & 27 & 29 & 27 & 28 & 27 & 29 \\
\hline-43 & 29 & 27 & 29 & 26 & 28 & 27 & 28 & 27 & 29 & 27 \\
\hline-40 & 28 & 27 & 28 & 27 & 28 & 28 & 27 & 28 & 28 & 28 \\
\hline .37 & 29 & 27 & 29 & 26 & 28 & 27 & 28 & 27 & 29 & 27 \\
\hline-35 & 27 & 29 & 26 & 30 & 27 & 29 & 27 & 28 & 27 & 29 \\
\hline-32 & 28 & 27 & 28 & 27 & 28 & 28 & 28 & 28 & 28 & 28 \\
\hline-29 & 27 & 29 & 26 & 30 & 27 & 29 & 27 & 28 & 27 & 29 \\
\hline-26 & 28 & 27 & 28 & 27 & 28 & 28 & 28 & 28 & 28 & 28 \\
\hline-23 & 29 & 27 & 29 & 26 & 29 & 27 & 28 & 27 & 28 & 27 \\
\hline-20 & 27 & 29 & 26 & 30 & 27 & 29 & 27 & 28 & 27 & 29 \\
\hline-17 & 29 & 27 & 28 & 26 & 28 & 27 & 28 & 27 & 28 & 27 \\
\hline-15 & 29 & 27 & 29 & 26 & 29 & 27 & 28 & 27 & 29 & 27 \\
\hline-12 & 28 & 27 & 28 & 26 & 29 & 27 & 29 & 27 & 29 & 27 \\
\hline-9 & 29 & 27 & 28 & 26 & 28 & 27 & 28 & 27 & 28 & 27 \\
\hline-6 & 29 & 27 & 29 & 26 & 29 & 27 & 29 & 27 & 29 & 27 \\
\hline-3 & 28 & 28 & 28 & 28 & 29 & 28 & 28 & 27 & 29 & 27 \\
\hline-0 & 29 & 27 & 29 & 26 & 29 & 27 & 28 & 27 & 28 & 27 \\
\hline 1 & 28 & 27 & 29 & 26 & 29 & 27 & 29 & 27 & 30 & 27 \\
\hline 4 & 28 & 28 & 28 & 27 & 29 & 28 & 29 & 27 & 30 & 27 \\
\hline 7 & 27 & 29 & 28 & 30 & 28 & 29 & 28 & 28 & 28 & 29 \\
\hline 10 & 28 & 27 & 30 & 27 & 28 & 28 & 29 & 28 & 29 & 27 \\
\hline 13 & 27 & 28 & 30 & 29 & 28 & 28 & 29 & 28 & 29 & 27 \\
\hline 16 & 28 & 28 & 29 & 30 & 27 & 29 & 28 & 29 & 28 & 29 \\
\hline
\end{tabular}


CLOSED DOOR SHIELDED FIRE TEST

THERMOCOUPLES AND DISKS AT SPRINKLER AND DETECTOR LOCATIONS - ${ }^{\circ} \mathrm{C}$

\begin{tabular}{|c|c|c|c|c|c|c|c|c|c|c|}
\hline TIME & T14 & D15 & T16 & D17 & T18 & D19 & T20 & D21 & T22 & $\mathrm{D} 23$ \\
\hline 19 & 28 & 28 & 30 & 29 & 28 & 28 & 28 & 28 & 29 & 27 \\
\hline 22 & 28 & 29 & 30 & 30 & 28 & 29 & 28 & 28 & 30 & 29 \\
\hline 24 & 29 & 29 & 31 & 30 & 28 & 29 & 29 & 29 & 29 & 30 \\
\hline 27 & 29 & 28 & 31 & 29 & 29 & 28 & 29 & 28 & 30 & 28 \\
\hline 30 & 29 & 29 & 31 & 31 & 29 & 29 & 29 & 29 & 29 & 30 \\
\hline 33 & 31 & 28 & 32 & 28 & 30 & 28 & 30 & 29 & 31 & 29 \\
\hline 36 & 31 & 28 & 34 & 28 & 31 & 28 & 31 & 28 & 32 & 28 \\
\hline 39 & 29 & 29 & 32 & 32 & 30 & 29 & 30 & 29 & 30 & 31 \\
\hline 41 & 31 & 28 & 34 & 29 & 31 & 29 & 32 & 29 & 32 & 29 \\
\hline 44 & 29 & 30 & 32 & 32 & 31 & 30 & 31 & 30 & 31 & 31 \\
\hline 47 & 39 & 28 & 35 & 30 & 33 & 29 & 32 & 29 & 32 & 29 \\
\hline 50 & 31 & 28 & 35 & 30 & 32 & 29 & 32 & 29 & 32 & 29 \\
\hline 53 & 31 & 30 & 34 & 32 & 32 & 30 & 32 & 30 & 32 & 30 \\
\hline 56 & 31 & 29 & 34 & 31 & 32 & 30 & 32 & 30 & 32 & 30 \\
\hline 59 & 31 & 31 & 34 & 33 & 32 & 31 & 32 & 31 & 32 & 31 \\
\hline 61 & 31 & 31 & 35 & 34 & 32 & 30 & 32 & 30 & 33 & 31 \\
\hline 64 & 31 & 31 & 34 & 35 & 31 & 31 & 32 & 31 & 32 & 32 \\
\hline 67 & 33 & 29 & 37 & 31 & 33 & 30 & 34 & 30 & 34 & 30 \\
\hline 70 & 31 & 31 & 35 & 35 & 32 & 32 & 32 & 32 & 32 & 33 \\
\hline 73 & 33 & 30 & 38 & 33 & 33 & 31 & 33 & 31 & 33 & 31 \\
\hline 76 & 33 & 30 & 39 & 34 & 32 & 31 & 33 & 32 & 33 & 33 \\
\hline 79 & 34 & 30 & 40 & 33 & 32 & 30 & 33 & 31 & 35 & 31 \\
\hline 82 & 33 & 30 & 38 & 34 & 33 & 39 & 33 & 32 & 35 & 33 \\
\hline 84 & 34 & 30 & 39 & 34 & 33 & 31 & 34 & 32 & 35 & 32 \\
\hline 87 & 35 & 31 & 39 & 34 & 34 & 31 & 35 & 31 & 37 & 32 \\
\hline
\end{tabular}


CLOSED DOOR SHIELDED FIRE TEST

THERMOCOUPLES AND DISKS AT SPRINKLER AND DETECTOR LOCATIONS - ${ }^{\circ} \mathrm{C}$

\begin{tabular}{|c|c|c|c|c|c|c|c|c|c|c|}
\hline TME & $T 14$ & D15 & T16 & D17 & T18 & D19 & T20 & D21 & T22 & $\mathrm{D} 23$ \\
\hline 90 & 34 & 31 & 39 & 34 & 33 & 31 & 34 & 32 & 37 & 33 \\
\hline 93 & 35 & 31 & 40 & 34 & 34 & 31 & 35 & 31 & 37 & 33 \\
\hline 96 & 33 & 32 & 38 & 37 & 34 & 32 & 34 & 33 & 36 & 35 \\
\hline 99 & 35 & 31 & 41 & 35 & 36 & 31 & 35 & 32 & 38 & 34 \\
\hline 101 & 35 & 32 & 41 & 35 & 36 & 32 & 36 & 32 & 39 & 34 \\
\hline 104 & 35 & 31 & 41 & 35 & 37 & 32 & 36 & 32 & 40 & 34 \\
\hline 107 & 36 & 32 & 41 & 36 & 36 & 33 & 37 & 33 & 39 & 35 \\
\hline 110 & 35 & 32 & 42 & 37 & 36 & 33 & 37 & 33 & 39 & 35 \\
\hline 113 & 33 & 34 & 39 & 40 & 34 & 34 & 35 & 34 & 38 & 38 \\
\hline 116 & 34 & 34 & 41 & 40 & 35 & 34 & 36 & 35 & 40 & 38 \\
\hline 118 & 35 & 33 & 43 & 38 & 37 & 33 & 38 & 34 & 40 & 36 \\
\hline 121 & 34 & 34 & 42 & 40 & 37 & 34 & 37 & 34 & 40 & 38 \\
\hline 124 & 34 & 34 & 41 & 41 & 37 & 35 & 37 & 35 & 41 & 39 \\
\hline 127 & 35 & 35 & 41 & 41 & 37 & 35 & 38 & 36 & 39 & 39 \\
\hline 130 & 35 & 35 & 42 & 41 & 37 & 35 & 38 & 36 & 40 & 40 \\
\hline 133 & 35 & 35 & 43 & 42 & 37 & 36 & 38 & 36 & 40 & 40 \\
\hline 136 & 37 & 34 & 45 & 40 & 39 & 35 & 40 & 35 & 42 & 38 \\
\hline 138 & 36 & 36 & 44 & 43 & 38 & 36 & 38 & 37 & 40 & 40 \\
\hline 141 & 38 & 34 & 46 & 39 & 39 & 35 & 41 & 35 & 43 & 38 \\
\hline 144 & 38 & 34 & 47 & 40 & 39 & 35 & 40 & 36 & 44 & 39 \\
\hline 147 & 38 & 35 & 46 & 41 & 39 & 35 & 40 & 36 & 42 & 39 \\
\hline 150 & 38 & 35 & 46 & 42 & 39 & 36 & 41 & 38 & 43 & 41 \\
\hline 153 & 40 & 35 & 46 & 41 & 40 & 36 & 42 & 37 & 43 & 40 \\
\hline 156 & 40 & 35 & 48 & 41 & 41 & 36 & 42 & 37 & 45 & 40 \\
\hline 158 & 40 & 35 & 48 & 41 & 40 & 36 & 42 & 38 & 47 & 41 \\
\hline
\end{tabular}


CLOSED DOOR SHIELDED FIRE TEST

THERMOCOUPLES AND DISKS AT SPRINKLER AND DETECTOR LOCATIONS - ${ }^{\circ} \mathrm{C}$

\begin{tabular}{|c|c|c|c|c|c|c|c|c|c|c|}
\hline TTME & T14 & D15 & T16 & D17 & T18 & D19 & $\mathrm{T} 20$ & D21 & T22 & D23 \\
\hline 161 & 41 & 36 & 46 & 41 & 40 & 37 & 41 & 38 & 45 & 41 \\
\hline 164 & 40 & 36 & 47 & 42 & 42 & 37 & 42 & 38 & 46 & 42 \\
\hline 167 & 40 & 36 & 49 & 42 & 42 & 37 & 43 & 38 & 47 & 41 \\
\hline 170 & 39 & 38 & 49 & 45 & 41 & 38 & 42 & 39 & 45 & 43 \\
\hline 173 & 40 & 38 & 48 & 45 & 42 & 38 & 42 & 40 & 45 & 42 \\
\hline 176 & 40 & 39 & 47 & 46 & 42 & 39 & 42 & 40 & 46 & 44 \\
\hline 179 & 40 & 38 & 49 & 46 & 41 & 39 & 43 & 41 & 45 & 45 \\
\hline 181 & 41 & 39 & 49 & 47 & 43 & 40 & 44 & 41 & 46 & 45 \\
\hline 184 & 41 & 39 & 47 & 47 & 43 & 40 & 43 & 41 & 49 & 45 \\
\hline 187 & 41 & 40 & 49 & 48 & 42 & 40 & 43 & 41 & 47 & 46 \\
\hline 190 & 41 & 40 & 49 & 48 & 42 & 40 & 44 & 41 & 48 & 46 \\
\hline 193 & 43 & 39 & 52 & 46 & 44 & 39 & 45 & 40 & 50 & 44 \\
\hline 196 & 42 & 41 & 49 & 49 & 43 & 41 & 44 & 42 & 48 & 47 \\
\hline 199 & 44 & 39 & 51 & 46 & 44 & 40 & 45 & 41 & 49 & 45 \\
\hline 201 & 44 & 40 & 51 & 48 & 43 & 41 & 45 & 42 & 49 & 47 \\
\hline 204 & 44 & 40 & 53 & 47 & 44 & 40 & 46 & 42 & 53 & 46 \\
\hline 207 & 43 & 41 & 52 & 50 & 44 & 42 & 45 & 43 & 50 & 49 \\
\hline 210 & 45 & 41 & 52 & 49 & 44 & 41 & 45 & 43 & 52 & 49 \\
\hline 213 & 44 & 41 & 52 & 50 & 45 & 42 & 46 & 43 & 53 & 49 \\
\hline 216 & 46 & 41 & 55 & 48 & 45 & 41 & 46 & 43 & 51 & 48 \\
\hline 219 & 46 & 41 & 57 & 49 & 48 & 41 & 48 & 43 & 53 & 48 \\
\hline 221 & 47 & 41 & 56 & 49 & 48 & 41 & 48 & 43 & 53 & 48 \\
\hline 224 & 47 & 41 & 57 & 49 & 47 & 42 & 48 & 43 & 53 & 49 \\
\hline 227 & 47 & 43 & 56 & 53 & 46 & 43 & 46 & 45 & 52 & 51 \\
\hline 230 & 48 & 42 & 58 & 50 & 48 & 42 & 48 & 44 & 56 & 50 \\
\hline
\end{tabular}


CLOSED DOOR SHIELDED FIRE TEST

THERMOCOUPLES AND DISKS AT SPRINKLER AND DETECTOR LOCATIONS - ${ }^{\circ} \mathrm{C}$

\begin{tabular}{|c|c|c|c|c|c|c|c|c|c|c|}
\hline TIME & T14 & D15 & $T 16$ & D17 & $T 18$ & D19 & $T 20$ & D21 & T22 & $\mathrm{D} 23$ \\
\hline 233 & 49 & 43 & 58 & 51 & 47 & 43 & 49 & 44 & 55 & 51 \\
\hline 236 & 48 & 43 & 59 & 52 & 48 & 43 & 49 & 45 & 55 & 51 \\
\hline 238 & 50 & 43 & 59 & 52 & 49 & 43 & 50 & 45 & 55 & 51 \\
\hline 241 & 50 & 44 & 61 & 53 & 50 & 44 & 51 & 45 & 55 & 51 \\
\hline 244 & 48 & 46 & 60 & 56 & 47 & 45 & 49 & 47 & 53 & 53 \\
\hline 247 & 49 & 46 & 61 & 57 & 47 & 45 & 48 & 47 & 55 & 53 \\
\hline 250 & 50 & 46 & 59 & 56 & 47 & 45 & 49 & 46 & 58 & 52 \\
\hline 253 & 51 & 45 & 61 & 54 & 47 & 44 & 51 & 46 & 57 & 52 \\
\hline 256 & 49 & 47 & 60 & 58 & 47 & 46 & 49 & 47 & 58 & 55 \\
\hline 258 & 50 & 47 & 61 & 58 & 49 & 46 & 53 & 48 & 58 & 55 \\
\hline 261 & 52 & 46 & 62 & 55 & 49 & 45 & 52 & 47 & 60 & 54 \\
\hline 264 & 52 & 47 & 63 & 56 & 50 & 45 & 53 & 48 & 61 & 54 \\
\hline 267 & 51 & 48 & 60 & 59 & 50 & 46 & 52 & 49 & 61 & 57 \\
\hline 270 & 51 & 49 & 62 & 60 & 49 & 47 & 51 & 49 & 58 & 57 \\
\hline 273 & 52 & 47 & 63 & 57 & 51 & 46 & 53 & 49 & 64 & 56 \\
\hline 275 & 51 & 49 & 63 & 59 & 50 & 47 & 52 & 49 & 65 & 58 \\
\hline 278 & 50 & 49 & 61 & 61 & 50 & 48 & 53 & 50 & 63 & 60 \\
\hline 281 & 51 & 50 & 62 & 61 & 50 & 48 & 54 & 51 & 63 & 60 \\
\hline 284 & 54 & 48 & 63 & 58 & 52 & 47 & 58 & 50 & 66 & 59 \\
\hline 287 & 52 & 49 & 64 & 60 & 53 & 48 & 55 & 52 & 64 & 61 \\
\hline 290 & 53 & 50 & 68 & 62 & 53 & 49 & 56 & 52 & 66 & 62 \\
\hline 293 & 56 & 49 & 68 & 60 & 55 & 48 & 57 & 51 & 68 & 61 \\
\hline 295 & 55 & 50 & 69 & 62 & 54 & 49 & 58 & 53 & 68 & 63 \\
\hline 298 & 55 & 51 & 69 & 64 & 55 & 50 & 58 & 53 & 69 & 64 \\
\hline 301 & 56 & 50 & 70 & 63 & 56 & 50 & 58 & 54 & 70 & 64 \\
\hline
\end{tabular}


CLOSED DOOR SHIELDED FIRE TEST

THERMOCOUPLES AND DISKS AT SPRINKLER AND DETECTOR LOCATIONS - ${ }^{\circ} \mathrm{C}$

\begin{tabular}{|c|c|c|c|c|c|c|c|c|c|c|}
\hline TIME & T14 & D15 & $T 16$ & 017 & T18 & D19 & T20 & D21 & T22 & $\mathrm{D} 23$ \\
\hline 304 & 57 & 51 & 72 & 63 & 56 & 50 & 61 & 54 & 71 & 64 \\
\hline 307 & 57 & 51 & 72 & 63 & 59 & 50 & 62 & 54 & 69 & 64 \\
\hline 310 & 58 & 51 & 72 & 64 & 58 & 51 & 63 & 56 & 72 & 66 \\
\hline 313 & 57 & 53 & 74 & 67 & 59 & 53 & 61 & 57 & 71 & 67 \\
\hline 315 & 59 & 52 & 78 & 66 & 61 & 52 & 64 & 56 & 73 & 66 \\
\hline 318 & 57 & 54 & 76 & 70 & 60 & 54 & 62 & 58 & 72 & 59 \\
\hline 321 & 60 & 55 & 76 & 71 & 60 & 54 & 63 & 59 & 73 & 69 \\
\hline 324 & 63 & 54 & 79 & 68 & 62 & 54 & 65 & 58 & 77 & 68 \\
\hline 327 & 60 & 56 & 79 & 72 & 62 & 55 & 66 & 59 & 79 & 70 \\
\hline 330 & 61 & 57 & $\pi$ & 73 & 63 & 56 & 64 & 60 & 76 & 72 \\
\hline 332 & 65 & 56 & 79 & 71 & 64 & 56 & 66 & 60 & 79 & 71 \\
\hline 335 & 63 & 58 & 83 & 76 & 62 & 57 & 66 & 62 & 78 & 74 \\
\hline 338 & 66 & 57 & 87 & 74 & 63 & 56 & 69 & 61 & 80 & 72 \\
\hline 341 & 64 & 60 & 85 & 78 & 62 & 58 & 66 & 63 & 80 & 76 \\
\hline 344 & 65 & 60 & 83 & 78 & 64 & 59 & 68 & 63 & 82 & 76 \\
\hline 347 & 65 & 61 & 86 & 79 & 64 & 59 & 68 & 63 & 86 & 78 \\
\hline 350 & 68 & 61 & 89 & 80 & 65 & 59 & 70 & 64 & 84 & $\pi$ \\
\hline 353 & 67 & 62 & 88 & 82 & 65 & 60 & 70 & 65 & 88 & 80 \\
\hline 355 & 70 & 61 & 91 & 79 & 68 & 59 & 73 & 65 & 89 & 79 \\
\hline 358 & 70 & 62 & 92 & 80 & 66 & 60 & 72 & 66 & 88 & 80 \\
\hline 361 & 70 & 62 & 93 & 81 & 71 & 61 & 73 & 66 & 92 & 81 \\
\hline 364 & 74 & 63 & 93 & 82 & 70 & 62 & 74 & 67 & 88 & 82 \\
\hline 367 & 73 & 63 & 44 & 64 & 71 & 62 & 76 & 68 & 90 & 82 \\
\hline 370 & 71 & .66 & 43 & 63 & 70 & 64 & 74 & 69 & 89 & 85 \\
\hline 372 & 74 & 65 & 46 & 58 & 73 & 63 & $\pi$ & 69 & 89 & 84 \\
\hline
\end{tabular}


CLOSED DOOR SHIELDED FIRE TEST

THERMOCOUPLES AND DISKS AT SPRINKLER AND DETECTOR LOCATIONS - ${ }^{\circ} \mathrm{C}$

\begin{tabular}{|c|c|c|c|c|c|c|c|c|c|c|}
\hline TME & $T 14$ & D15 & $T 16$ & D17 & $T 18$ & D19 & T20 & D21 & T22 & $\mathrm{D} 23$ \\
\hline 375 & 75 & 67 & 78 & 60 & 71 & 64 & 77 & 70 & 92 & 86 \\
\hline 378 & 74 & 67 & 91 & 64 & 74 & 65 & 78 & 71 & 93 & 88 \\
\hline 381 & 73 & 66 & 91 & 65 & 78 & 65 & 80 & 71 & 96 & 87 \\
\hline 384 & 71 & 68 & 93 & 71 & 75 & 67 & 77 & 72 & 96 & 89 \\
\hline 387 & 73 & 69 & 98 & 75 & 74 & 67 & 77 & 72 & 94 & 89 \\
\hline 390 & 76 & 68 & 97 & 74 & 76 & 67 & 80 & 72 & 99 & 88 \\
\hline 392 & 74 & 70 & 91 & 79 & 75 & 69 & 80 & 74 & 96 & 91 \\
\hline 395 & 73 & 69 & 93 & 79 & 74 & 69 & 77 & 74 & 95 & 90 \\
\hline 398 & 73 & 70 & 96 & 83 & 74 & 69 & 78 & 75 & 93 & 91 \\
\hline 401 & 74 & 71 & 94 & 84 & 73 & 70 & 78 & 75 & 99 & 93 \\
\hline 404 & 73 & 71 & 94 & 85 & 75 & 70 & 78 & 75 & 97 & 93 \\
\hline 407 & 76 & 70 & 94 & 83 & 76 & 70 & 82 & 75 & 93 & 92 \\
\hline 409 & 75 & 72 & 90 & 87 & 74 & 71 & 79 & 76 & 89 & 92 \\
\hline 412 & 77 & 70 & 92 & 84 & 76 & 69 & 80 & 75 & 91 & 90 \\
\hline 415 & 75 & 71 & 89 & 86 & 76 & 71 & 78 & 76 & 89 & 92 \\
\hline 418 & 76 & 70 & 88 & 84 & 75 & 70 & 79 & 75 & 88 & 90 \\
\hline 421 & 74 & 70 & 88 & 83 & 77 & 70 & 79 & 75 & 88 & 89 \\
\hline 424 & 74 & 70 & 91 & 84 & 78 & 71 & 78 & 75 & 94 & 90 \\
\hline 427 & 73 & 72 & 90 & 87 & $\pi$ & 72 & 79 & 77 & 93 & 92 \\
\hline 429 & 74 & 70 & 95 & 85 & 77 & 71 & 80 & 75 & 91 & 89 \\
\hline 432 & 74 & 72 & 92 & 88 & 75 & 73 & 80 & 77 & 91 & 92 \\
\hline 435 & 75 & 73 & 94 & 89 & 75 & 73 & 81 & $\pi$ & 93 & 92 \\
\hline 438 & 76 & 71 & 95 & 87 & 76 & 72 & 82 & 76 & 94 & 90 \\
\hline 441 & 74 & 72 & 94 & 89 & 74 & 72 & 81 & $\pi$ & 95 & 91 \\
\hline 444 & 75 & 73 & 94 & 91 & 76 & 73 & 80 & 78 & 92 & 92 \\
\hline
\end{tabular}


CLOSED DOOR SHIELDED FIRE TEST

THERMOCOUPLES AND DISKS AT SPRINKLER AND DETECTOR LOCATIONS . ${ }^{\circ} \mathrm{C}$

\begin{tabular}{|c|c|c|c|c|c|c|c|c|c|c|}
\hline TME & T14 & 015 & T16 & D17 & T18 & D19 & T20 & $\mathrm{D} 21$ & T22 & $\mathrm{D} 23$ \\
\hline 447 & 75 & 74 & 93 & 91 & 77 & 73 & 80 & 78 & 91 & 92 \\
\hline 449 & 75 & 74 & 92 & 91 & 75 & 74 & 82 & 79 & 93 & 93 \\
\hline 452 & 76 & 72 & 100 & 89 & 77 & 73 & 84 & 79 & 97 & 93 \\
\hline 455 & 77 & 74 & 95 & 92 & 78 & 74 & 84 & 80 & 94 & 94 \\
\hline 458 & 78 & 72 & 97 & 89 & 81 & 73 & 84 & 78 & 97 & 92 \\
\hline 461 & 75 & 74 & 93 & 93 & 80 & 75 & 84 & 80 & 96 & 94 \\
\hline 464 & 76 & 74 & 94 & 92 & 78 & 74 & 84 & 80 & 94 & 94 \\
\hline 466 & 77 & 73 & 99 & 91 & 79 & 74 & 86 & 80 & 99 & 95 \\
\hline 469 & 28 & 73 & 101 & 91 & 80 & 74 & 86 & 80 & 97 & 93 \\
\hline 472 & 79 & 73 & 101 & 92 & 79 & 74 & 88 & 80 & 100 & 94 \\
\hline 475 & 80 & 74 & 103 & 93 & 80 & 74 & 87 & 81 & 103 & 95 \\
\hline 478 & 80 & 74 & 104 & 93 & 81 & 74 & 87 & 81 & 101 & 95 \\
\hline 481 & 79 & 76 & 101 & 96 & 81 & 76 & 88 & 83 & 97 & 96 \\
\hline 484 & 80 & 76 & 101 & 98 & 84 & $\pi$ & 87 & 83 & 96 & 97 \\
\hline 486 & 80 & 76 & 105 & 98 & 83 & $\pi 7$ & 86 & 83 & 99 & 97 \\
\hline 489 & 82 & 76 & 107 & 98 & 84 & $\pi 7$ & 90 & 83 & 103 & 97 \\
\hline 492 & 81 & $\pi$ & 104 & 99 & 83 & 78 & 87 & 84 & 102 & 99 \\
\hline 495 & 81 & 77 & 102 & 98 & 85 & 78 & 90 & 84 & 102 & 97 \\
\hline 498 & 83 & 78 & 104 & 101 & 84 & 79 & 90 & 85 & 106 & 101 \\
\hline 501 & 84 & $\pi$ & 105 & 97 & 86 & 78 & 93 & 85 & 104 & 99 \\
\hline 504 & 82 & 78 & 104 & 101 & 85 & 79 & 91 & 86 & 103 & 101 \\
\hline 506 & 83 & $\pi$ & 107 & 99 & 87 & 79 & 93 & 86 & 105 & 100 \\
\hline 509 & 83 & 78 & 110 & 104 & 86 & 80 & 92 & 87 & 104 & 102 \\
\hline 512 & 86 & 80 & 110 & 103 & 86 & 80 & 94 & 87 & 105 & 101 \\
\hline 515 & 88 & 81 & 109 & 104 & 85 & 81 & 93 & 88 & 106 & 103 \\
\hline
\end{tabular}


CLOSED DOOR SHIELDED FIRE TEST

THERMOCOUPLES AND DISKS AT SPRINKLER AND DETECTOR LOCATIONS $-{ }^{\circ} \mathrm{C}$

\begin{tabular}{|c|c|c|c|c|c|c|c|c|c|c|}
\hline TIME & T14 & D15 & T16 & D17 & T18 & D19 & T20 & $\mathrm{D} 21$ & T22 & $\mathrm{D} 23$ \\
\hline 518 & 87 & 82 & 115 & 106 & 88 & 82 & 94 & 89 & 107 & 104 \\
\hline 521 & 90 & 82 & 116 & 105 & 88 & 81 & 96 & 89 & 109 & 102 \\
\hline 523 & 89 & 82 & 116 & 105 & 89 & 82 & 96 & 89 & 110 & 103 \\
\hline 526 & 87 & 83 & 118 & 108 & 91 & 83 & 95 & 89 & 113 & 105 \\
\hline 529 & 91 & 83 & 124 & 107 & 93 & 83 & 97 & 89 & 112 & 104 \\
\hline 532 & 94 & 84 & 121 & 108 & 96 & 84 & 99 & 90 & 111 & 105 \\
\hline 535 & 90 & 85 & 120 & 112 & 91 & 85 & 96 & 92 & 112 & 108 \\
\hline 538 & 49 & 84 & 126 & 111 & 91 & 84 & 102 & 91 & 112 & 105 \\
\hline 540 & 60 & 85 & 124 & 112 & 94 & 84 & 101 & 92 & 116 & 107 \\
\hline 543 & 67 & 87 & 127 & 115 & 94 & 86 & 102 & 94 & 111 & 109 \\
\hline 546 & 78 & 88 & 126 & 117 & 92 & 86 & 103 & 95 & 118 & 110 \\
\hline 549 & $\pi$ & 89 & 127 & 119 & 95 & 87 & 100 & 96 & 114 & 111 \\
\hline 552 & 82 & 90 & 129 & 118 & 99 & 88 & 102 & 95 & 115 & 110 \\
\hline 555 & 84 & 90 & 125 & 119 & 99 & 89 & 106 & 97 & 116 & 112 \\
\hline 558 & 91 & 91 & 126 & 120 & 100 & 90 & 107 & 98 & 119 & 113 \\
\hline 560 & 98 & 92 & 136 & 121 & 103 & 90 & 108 & 98 & 123 & 113 \\
\hline 563 & 99 & 93 & 135 & 122 & 103 & 91 & 107 & 99 & 121 & 114 \\
\hline 566 & 102 & 94 & 132 & 122 & 104 & 92 & 107 & 99 & 124 & 115 \\
\hline 569 & 101 & 93 & 132 & 121 & 105 & 92 & 112 & 100 & 126 & 116 \\
\hline 572 & 102 & 95 & 136 & 124 & 102 & 94 & 110 & 101 & 122 & 117 \\
\hline 575 & 103 & 96 & 135 & 125 & 104 & 94 & 108 & 101 & 122 & 117 \\
\hline 578 & 103 & 95 & 136 & 124 & 104 & 94 & 110 & 101 & 127 & 118 \\
\hline 580 & 103 & 97 & 138 & 127 & 104 & 95 & 109 & 102 & 123 & 120 \\
\hline 583 & 103 & 97 & 133 & 127 & 106 & 96 & 112 & 103 & 125 & 120 \\
\hline 586 & 103 & 97 & 139 & 127 & 108 & 96 & 113 & 104 & 129 & 121 \\
\hline
\end{tabular}


CLOSED DOOR SHIELDED FIRE TEST

THERMOCOUPLES AND DISKS AT SPRINKLER AND DETECTOR LOCATIONS - ${ }^{\circ} \mathrm{C}$

\begin{tabular}{|c|c|c|c|c|c|c|c|c|c|c|}
\hline TIME & T14 & D15 & T16 & D17 & T18 & D19 & T20 & $\mathrm{D} 21$ & T22 & 023 \\
\hline 589 & 105 & 98 & 138 & 126 & 110 & 97 & 116 & 104 & 128 & 120 \\
\hline 592 & 105 & 98 & 137 & 127 & 112 & 98 & 113 & 105 & 129 & 121 \\
\hline 595 & 102 & 99 & 141 & 131 & 110 & 100 & 112 & 106 & 132 & 125 \\
\hline 597 & 103 & 100 & 139 & 132 & 109 & 100 & 113 & 106 & 131 & 125 \\
\hline 600 & 106 & 101 & 136 & 132 & 111 & 101 & 117 & 107 & 130 & 125 \\
\hline 603 & 108 & 100 & 150 & 131 & 112 & 101 & 114 & 107 & 136 & 125 \\
\hline 606 & 110 & 103 & 144 & 135 & 114 & 102 & 116 & 109 & 133 & 128 \\
\hline 609 & 110 & 103 & 148 & 135 & 115 & 103 & 120 & 110 & 132 & 126 \\
\hline 612 & 109 & 103 & 143 & 136 & 117 & 104 & 119 & 111 & 130 & 128 \\
\hline 615 & 112 & 103 & 147 & 135 & 118 & 104 & 117 & 110 & 132 & 127 \\
\hline 617 & 113 & 105 & 150 & 139 & 115 & 106 & 120 & 112 & 132 & 130 \\
\hline 620 & 117 & 105 & 151 & 138 & 115 & 105 & 121 & 112 & 136 & 129 \\
\hline 623 & 117 & 106 & 157 & 139 & 117 & 105 & 117 & 112 & 140 & 130 \\
\hline 626 & 116 & 108 & 156 & 144 & 116 & 108 & 122 & 114 & 141 & 133 \\
\hline 629 & 117 & 107 & 152 & 141 & 117 & 107 & 122 & 114 & 142 & 132 \\
\hline 632 & 117 & 108 & 156 & 143 & 119 & 108 & 122 & 115 & 144 & 135 \\
\hline 635 & 119 & 109 & 162 & 144 & 122 & 108 & 122 & 114 & 145 & 134 \\
\hline 637 & 122 & 110 & 155 & 144 & 121 & 109 & 124 & 115 & 145 & 135 \\
\hline 640 & 120 & 111 & 163 & 148 & 117 & 110 & 125 & 117 & 141 & 137 \\
\hline 643 & 121 & 113 & 156 & 148 & 121 & 111 & 127 & 117 & 148 & 137 \\
\hline 646 & 123 & 113 & 156 & 149 & 124 & 112 & 124 & 118 & 143 & 139 \\
\hline 649 & 123 & 115 & 156 & 150 & 122 & 113 & 127 & 119 & 144 & 139 \\
\hline 652 & 123 & 115 & 156 & 150 & 124 & 113 & 128 & 120 & 141 & 139 \\
\hline 654 & 124 & 114 & 164 & 149 & 123 & 113 & 124 & 119 & 142 & 137 \\
\hline 657 & 123 & 115 & 165 & 151 & 124 & 114 & 124 & 119 & 142 & 139 \\
\hline
\end{tabular}


THERMOCOUPLES AND DISKS AT SPRINKLER AND DETECTOR LOCATIONS - ${ }^{\circ} \mathrm{C}$

\begin{tabular}{|c|c|c|c|c|c|c|c|c|c|c|}
\hline TME & T14 & D15 & $T_{16}$ & D17 & $T_{18}$ & D19 & T2O & D21 & T22 & D23 \\
\hline 660 & 124 & 117 & 164 & 155 & 123 & 115 & 125 & 121 & 146 & 141 \\
\hline 663 & 125 & 116 & 162 & 152 & 126 & 114 & 128 & 120 & 141 & 138 \\
\hline 666 & 125 & 118 & 165 & 156 & 124 & 116 & 128 & 122 & 142 & 141 \\
\hline 669 & 125 & 118 & 167 & 156 & 128 & 116 & 131 & 123 & 148 & 141 \\
\hline 672 & 124 & 118 & 166 & 157 & 127 & 117 & 131 & 123 & 149 & 142 \\
\hline 674 & 125 & 119 & 165 & 157 & 126 & 117 & 133 & 124 & 146 & 142 \\
\hline 677 & 121 & 119 & 159 & 158 & 128 & 119 & 130 & 125 & 144 & 143 \\
\hline 680 & 127 & 120 & 162 & 158 & 125 & 118 & 128 & 124 & 145 & 143 \\
\hline 683 & 128 & 120 & 168 & 159 & 128 & 120 & 129 & 125 & 143 & 143 \\
\hline 686 & 131 & 120 & 160 & 155 & 129 & 119 & 133 & 124 & 146 & 142 \\
\hline 689 & 127 & 122 & 161 & 158 & 128 & 120 & 134 & 126 & 148 & 145 \\
\hline 692 & 127 & 121 & 164 & 156 & 129 & 120 & 133 & 126 & 148 & 144 \\
\hline 694 & 127 & 120 & 163 & 156 & 128 & 120 & 131 & 126 & 153 & 144 \\
\hline 697 & 101 & 104 & 110 & 158 & 46 & 79 & 94 & 94 & 146 & 145 \\
\hline 700 & 53 & 66 & 114 & 150 & 49 & 62 & 103 & 93 & 132 & 140 \\
\hline 703 & 49 & 60 & 117 & 148 & 48 & 62 & 105 & 96 & 118 & 139 \\
\hline 706 & 45 & 54 & 93 & 143 & 52 & 60 & 105 & 97 & 119 & 134 \\
\hline 709 & 46 & 47 & 99 & 136 & 54 & 56 & 103 & 97 & 110 & 130 \\
\hline 711 & 42 & 49 & 85 & 133 & 51 & 58 & 93 & 97 & 103 & 128 \\
\hline 714 & 44 & 44 & 89 & 125 & 54 & 53 & 90 & 95 & 102 & 122 \\
\hline 717 & 45 & 44 & 86 & 122 & 52 & 54 & 85 & 94 & 94 & 119 \\
\hline 720 & 45 & 42 & $\pi 7$ & 117 & 53 & 53 & 82 & 92 & 88 & 114 \\
\hline 723 & 41 & 45 & 87 & 114 & 47 & 52 & 81 & 91 & 94 & 112 \\
\hline 726 & 41 & 45 & 87 & 112 & 48 & 52 & 81 & 90 & 91 & 109 \\
\hline 728 & 44 & 41 & 83 & 106 & 50 & 50 & 75 & 87 & 86 & 104 \\
\hline
\end{tabular}


THERMOCOUPLES AND DISKS AT SPRINKLER AND DETECTOR LOCATIONS $-{ }^{\circ} \mathrm{C}$

\begin{tabular}{|c|c|c|c|c|c|c|c|c|c|c|}
\hline TIME & $T 14$ & 015 & $T 16$ & 017 & T18 & D19 & T20 & D21 & T22 & $\mathrm{D} 23$ \\
\hline 731 & 41 & 44 & 79 & 106 & 47 & 52 & 77 & 87 & 88 & 103 \\
\hline 734 & 43 & 41 & 70 & 100 & 50 & 49 & 78 & 84 & 86 & 98 \\
\hline 737 & 40 & 44 & 74 & 98 & 45 & 49 & 75 & 84 & 82 & 96 \\
\hline 740 & 40 & 44 & 83 & 99 & 47 & 50 & 75 & 83 & 83 & 96 \\
\hline 743 & 40 & 44 & 62 & 95 & 45 & 50 & 70 & 81 & 77 & 93 \\
\hline 746 & 44 & 40 & 65 & 89 & 49 & 45 & 70 & 78 & 79 & 89 \\
\hline 748 & 44 & 40 & 65 & 87 & 51 & 46 & 70 & 77 & 74 & 86 \\
\hline 751 & 39 & 44 & 77 & 90 & 45 & 50 & 64 & 75 & 69 & 86 \\
\hline 754 & 44 & 40 & 76 & 84 & 49 & 45 & 66 & 74 & 70 & 82 \\
\hline 757 & 41 & 43 & 67 & 84 & 49 & 48 & 68 & 74 & 70 & 82 \\
\hline 760 & 43 & 39 & 63 & 76 & 51 & 45 & 66 & 71 & 67 & 78 \\
\hline 763 & 43 & 40 & 57 & 74 & 47 & 44 & 63 & 70 & 64 & 76 \\
\hline 766 & 39 & 43 & 55 & 75 & 46 & 48 & 64 & 70 & 65 & 77 \\
\hline 768 & 38 & 43 & 54 & 73 & 44 & 47 & 63 & 70 & 67 & 76 \\
\hline 771 & 41 & 41 & 51 & 70 & 46 & 45 & 62 & 68 & 63 & 73 \\
\hline 774 & 43 & 39 & 55 & 67 & 47 & 43 & 60 & 66 & 62 & 71 \\
\hline$\pi 77$ & 39 & 43 & 45 & 68 & 42 & 46 & 58 & 66 & 61 & 72 \\
\hline 780 & 41 & 40 & 51 & 63 & 46 & 44 & 58 & 64 & 63 & 69 \\
\hline 783 & 41 & 39 & 44 & 60 & 46 & 44 & 60 & 64 & 61 & 70 \\
\hline 786 & 42 & 38 & 47 & 55 & 46 & 42 & 55 & 62 & 60 & 66 \\
\hline 788 & 41 & 40 & 43 & 53 & 45 & 44 & 57 & 62 & 63 & 67 \\
\hline 791 & 41 & 38 & 42 & 51 & 45 & 41 & 57 & 60 & 59 & 64 \\
\hline 794 & 38 & 42 & 39 & 53 & 40 & 43 & 53 & 61 & 59 & 66 \\
\hline 797 & 41 & 38 & 43 & 48 & 46 & 40 & 57 & 59 & 61 & 63 \\
\hline 800 & 37 & 42 & 39 & 50 & 38 & 45 & 54 & 60 & 60 & 65 \\
\hline
\end{tabular}


CLOSED DOOR SHIELDED FIRE TEST

THERMOCOUPLES AND DISKS AT SPRINKLER AND DETECTOR LOCATIONS - ${ }^{\circ} \mathrm{C}$

\begin{tabular}{|c|c|c|c|c|c|c|c|c|c|c|}
\hline TME & $T 14$ & D15 & T16 & D17 & $T 18$ & Dis & T20 & D21 & T22 & $\mathrm{D} 23$ \\
\hline 803 & 37 & 41 & 40 & 47 & 41 & 44 & 54 & 59 & 58 & 64 \\
\hline 806 & 37 & 41 & 39 & 47 & 40 & 44 & 52 & 58 & 55 & 63 \\
\hline 808 & 37 & 41 & 41 & 46 & 39 & 43 & 55 & 58 & 53 & 61 \\
\hline 811 & 37 & 41 & 41 & 47 & 40 & 43 & 56 & 58 & 61 & 62 \\
\hline 814 & 39 & 38 & 42 & 43 & 41 & 40 & 54 & 57 & 58 & 61 \\
\hline 817 & 40 & 37 & 45 & 43 & 44 & 39 & 55 & 56 & 57 & 59 \\
\hline 820 & 40 & 37 & 47 & 46 & 42 & 39 & 58 & 56 & 65 & 60 \\
\hline 823 & 36 & 40 & 44 & 49 & 41 & 42 & 58 & 57 & 61 & 61 \\
\hline 826 & 36 & 40 & 46 & 51 & 37 & 41 & 54 & 57 & 61 & 61 \\
\hline 829 & 39 & 36 & 41 & 45 & 44 & 38 & 56 & 55 & 60 & 59 \\
\hline 832 & 35 & 40 & 40 & 47 & 40 & 43 & 56 & 57 & 57 & 60 \\
\hline 834 & 35 & 40 & 38 & 46 & 39 & 42 & 52 & 56 & 56 & 60 \\
\hline 837 & 36 & 39 & 38 & 44 & 41 & 43 & 55 & 56 & 58 & 60 \\
\hline 840 & 35 & 40 & 37 & 44 & 38 & 43 & 51 & 55 & 55 & 59 \\
\hline 843 & 36 & 39 & 39 & 43 & 39 & 41 & 51 & 54 & 56 & 59 \\
\hline 846 & 38 & 38 & 40 & 40 & 41 & 39 & 55 & 54 & 59 & 57 \\
\hline 849 & 37 & 38 & 40 & 40 & 41 & 39 & 52 & 54 & 58 & 57 \\
\hline 852 & 36 & 39 & 38 & 41 & 40 & 41 & 53 & 54 & 55 & 58 \\
\hline 854 & 39 & 35 & 42 & 39 & 41 & 37 & 54 & 53 & 54 & 56 \\
\hline 857 & 38 & 35 & 41 & 38 & 40 & 36 & 53 & 53 & 53 & 55 \\
\hline 860 & 39 & 35 & 40 & 38 & 41 & 36 & 53 & 53 & 52 & 55 \\
\hline 863 & 38 & 35 & 40 & 38 & 42 & 37 & 52 & 52 & 51 & 54 \\
\hline 866 & 39 & 35 & 41 & 38 & 41 & 36 & 52 & 52 & 51 & 54 \\
\hline 869 & 39 & 35 & 41 & 37 & 38 & 35 & 50 & 51 & 51 & 53 \\
\hline 872 & 39 & 35 & 41 & 37 & 41 & 35 & 50 & 51 & 51 & 52 \\
\hline
\end{tabular}


THERMOCOUPLES AND DISKS AT SPRINKLER AND DETECTOR LOCATIONS - ${ }^{\circ} \mathrm{C}$

\begin{tabular}{|c|c|c|c|c|c|c|c|c|c|c|}
\hline TIME & T14 & D15 & T16 & D17 & T18 & D19 & T20 & $D 21$ & T22 & $\mathrm{D} 23$ \\
\hline 875 & 34 & 39 & 37 & 41 & 35 & 40 & 48 & 52 & 50 & 54 \\
\hline 877 & 37 & 36 & 39 & 38 & 41 & 37 & 50 & 51 & 54 & 53 \\
\hline 880 & 34 & 39 & 36 & 41 & 38 & 41 & 48 & 51 & 49 & 53 \\
\hline 883 & 34 & 39 & 36 & 41 & 37 & 41 & 47 & 51 & 51 & 54 \\
\hline 886 & 38 & 36 & 40 & 38 & 40 & 37 & 47 & 50 & 49 & 51 \\
\hline 889 & 35 & 39 & 36 & 41 & 36 & 40 & 45 & 50 & 45 & 52 \\
\hline 892 & 34 & 39 & 36 & 41 & 36 & 40 & 44 & 50 & 45 & 51 \\
\hline 895 & 38 & 36 & 39 & 38 & 40 & 37 & 45 & 48 & 46 & 49 \\
\hline 898 & 35 & 39 & 36 & 40 & 37 & 40 & 45 & 49 & 46 & 51 \\
\hline 901 & 35 & 39 & 36 & 41 & 37 & 41 & 45 & 49 & 46 & 51 \\
\hline 903 & 38 & 35 & 39 & 36 & 41 & 37 & 46 & 47 & 46 & 48 \\
\hline 906 & 38 & 34 & 39 & 36 & 41 & 36 & 46 & 46 & 46 & 48 \\
\hline 909 & 38 & 35 & 39 & 36 & 40 & 36 & 44 & 46 & 44 & 47 \\
\hline 912 & 38 & 35 & 38 & 36 & 40 & 36 & 44 & 46 & 46 & 47 \\
\hline 915 & 38 & 34 & 39 & 35 & 40 & 35 & 44 & 45 & 45 & 46 \\
\hline 918 & 38 & 34 & 39 & 35 & 38 & 34 & 44 & 45 & 46 & 46 \\
\hline 921 & 38 & 34 & 39 & 35 & 39 & 34 & 46 & 45 & 48 & 46 \\
\hline 924 & 38 & 34 & 39 & 35 & 41 & 35 & 45 & 45 & 46 & 46 \\
\hline 926 & 37 & 34 & 39 & 35 & 41 & 35 & 46 & 45 & 47 & 46 \\
\hline 929 & 37 & 34 & 39 & 35 & 40 & 35 & 44 & 44 & 45 & 45 \\
\hline 932 & 33 & 38 & 35 & 39 & 35 & 38 & 43 & 45 & 45 & 47 \\
\hline 935 & 33 & 38 & 34 & 39 & 35 & 39 & 43 & 46 & 45 & 47 \\
\hline 938 & 34 & 36 & 36 & 37 & 37 & 36 & 42 & 44 & 45 & 45 \\
\hline 941 & 32 & 38 & 33 & 39 & 36 & 39 & 43 & 45 & 43 & 47 \\
\hline 944 & 33 & 38 & 34 & 39 & 36 & 40 & 43 & 45 & 42 & 46 \\
\hline
\end{tabular}


CLOSED DOOR SHIELDED FIRE TEST

THERMOCOUPLES AND DISKS AT SPRINKLER AND DETECTOR LOCATIONS - ${ }^{\circ} \mathrm{C}$

\begin{tabular}{|c|c|c|c|c|c|c|c|c|c|c|}
\hline TIME & $\mathrm{T}_{14}$ & D15 & T16 & D17 & T18 & 019 & T2O & $D 21$ & T22 & D23 \\
\hline 947 & 35 & 36 & 36 & 37 & 37 & 38 & 44 & 45 & 43 & 46 \\
\hline 950 & 36 & 34 & 37 & 35 & 36 & 34 & 44 & 44 & 46 & 44 \\
\hline 953 & 36 & 34 & 37 & 34 & 36 & 34 & 44 & 44 & 45 & 44 \\
\hline 956 & 33 & 37 & 33 & 38 & 35 & 37 & 42 & 45 & 43 & 46 \\
\hline 959 & 32 & 37 & 34 & 39 & 35 & 38 & 42 & 44 & 42 & 46 \\
\hline 961 & 36 & 33 & 38 & 34 & 37 & 33 & 44 & 43 & 44 & 44 \\
\hline 964 & 32 & 37 & 34 & 38 & 33 & 37 & 42 & 44 & 43 & 46 \\
\hline 967 & 36 & 33 & 37 & 34 & 37 & 34 & 41 & 43 & 43 & 43 \\
\hline 970 & 36 & 33 & 37 & 34 & 38 & 34 & 43 & 42 & 44 & 43 \\
\hline 973 & 36 & 33 & 38 & 34 & 38 & 34 & 42 & 42 & 43 & 43 \\
\hline 976 & 36 & 32 & 38 & 33 & 39 & 34 & 43 & 42 & 44 & 43 \\
\hline 979 & 36 & 32 & 38 & 34 & 39 & 34 & 43 & 42 & 44 & 43 \\
\hline 981 & 32 & 36 & 33 & 37 & 35 & 37 & 41 & 43 & 42 & 44 \\
\hline 984 & 32 & 36 & 33 & 37 & 34 & 38 & 41 & 43 & 41 & 44 \\
\hline 987 & 36 & 33 & 37 & 33 & 38 & 34 & 43 & 42 & 44 & 42 \\
\hline 990 & 32 & 36 & 34 & 37 & 35 & 37 & 42 & 43 & 43 & 44 \\
\hline 993 & 32 & 36 & 34 & 38 & 33 & 37 & 42 & 43 & 44 & 45 \\
\hline 996 & 32 & 36 & 34 & 37 & 33 & 36 & 43 & 43 & 44 & 45 \\
\hline 999 & 33 & 36 & 33 & 37 & 32 & 36 & 42 & 43 & 44 & 45 \\
\hline 1002 & 32 & 36 & 33 & 37 & 32 & 36 & 42 & 43 & 43 & 45 \\
\hline 1004 & 36 & 32 & 37 & 33 & 39 & 32 & 44 & 42 & 45 & 43 \\
\hline 1007 & 31 & 36 & 33 & 38 & 34 & 37 & 42 & 43 & 43 & 45 \\
\hline 1010 & 35 & 33 & 36 & 34 & 37 & 35 & 42 & 43 & 43 & 44 \\
\hline 1013 & 32 & 35 & 34 & 37 & 33 & 37 & 41 & 43 & 41 & 44 \\
\hline 1016 & 32 & 36 & 33 & 37 & 32 & 36 & 41 & 43 & 41 & 44 \\
\hline
\end{tabular}


THERMOCOUPLES AND DISKS AT SPRINKLER AND DETECTOR LOCATIONS - ${ }^{\circ} \mathrm{C}$

\begin{tabular}{|c|c|c|c|c|c|c|c|c|c|c|}
\hline TIME & T14 & D15 & T16 & D17 & $\mathrm{T} 18$ & D19 & T20 & $\mathrm{D} 21$ & T22 & $\mathrm{D} 23$ \\
\hline 1019 & 34 & 34 & 35 & 36 & 34 & 35 & 41 & 42 & 41 & 44 \\
\hline 1022 & 36 & 32 & 37 & 33 & 36 & 31 & 42 & 41 & 41 & 41 \\
\hline 1025 & 35 & 32 & 37 & 33 & 37 & 33 & 42 & 41 & 41 & 43 \\
\hline 1027 & 33 & 35 & 34 & 36 & 35 & 35 & 40 & 42 & 44 & 44 \\
\hline 1030 & 35 & 33 & 36 & 34 & 36 & 32 & 43 & 41 & 44 & 42 \\
\hline 1033 & 32 & 36 & 33 & 37 & 34 & 36 & 40 & 42 & 41 & 44 \\
\hline 1036 & 32 & 36 & 33 & 37 & 34 & 37 & 40 & 42 & 41 & 43 \\
\hline 1039 & 32 & 36 & 33 & 37 & 33 & 36 & 41 & 42 & 43 & 43 \\
\hline 1042 & 32 & 36 & 33 & 37 & 33 & 37 & 40 & 42 & 41 & 44 \\
\hline 1045 & 32 & 36 & 32 & 37 & 31 & 36 & 41 & 42 & 41 & 43 \\
\hline 1048 & 31 & 36 & 33 & 37 & 32 & 35 & 40 & 42 & 42 & 43 \\
\hline 1050 & 31 & 36 & 32 & 37 & 33 & 36 & 41 & 42 & 42 & 43 \\
\hline 1053 & 35 & 32 & 36 & 33 & 37 & 33 & 42 & 41 & 43 & 41 \\
\hline 1056 & 32 & 35 & 33 & 37 & 33 & 36 & 41 & 42 & 41 & 43 \\
\hline 1059 & 32 & 35 & 33 & 37 & 34 & 37 & 40 & 42 & 40 & 43 \\
\hline 1062 & 32 & 35 & 32 & 37 & 33 & 36 & 40 & 42 & 40 & 43 \\
\hline 1065 & 31 & 36 & 32 & 37 & 31 & 36 & 40 & 42 & 41 & 43 \\
\hline 1068 & 35 & 32 & 36 & 32 & 37 & 32 & 41 & 41 & 42 & 41 \\
\hline 1071 & 35 & 32 & 35 & 33 & 35 & 33 & 41 & 41 & 41 & 41 \\
\hline 1073 & 31 & 36 & 32 & 37 & 33 & 36 & 43 & 42 & 44 & 44 \\
\hline 1076 & 35 & 32 & 37 & 33 & 38 & 33 & 45 & 41 & 44 & 41 \\
\hline 1079 & 34 & 33 & 35 & 34 & 36 & 35 & 43 & 42 & 43 & 43 \\
\hline 1082 & 35 & 32 & 35 & 33 & 37 & 34 & 43 & 42 & 43 & 41 \\
\hline 1085 & 35 & 31 & 36 & 32 & 37 & 33 & 41 & 41 & 42 & 41 \\
\hline 1088 & 35 & 31 & 36 & 32 & 38 & 33 & 43 & 41 & 45 & 41 \\
\hline
\end{tabular}


CLOSED DOOR SHIELDED FIRE TEST

THERMOCOUPLES AND DISKS AT SPRINKLER AND DETECTOR LOCATIONS - ${ }^{\circ} \mathrm{C}$

\begin{tabular}{|c|c|c|c|c|c|c|c|c|c|c|}
\hline TIME & T14 & D15 & T16 & D17 & T18 & D19 & $T 20$ & D21 & T22 & $\mathrm{D} 23$ \\
\hline 1091 & 31 & 36 & 33 & 37 & 34 & 37 & 42 & 42 & 42 & 43 \\
\hline 1094 & 33 & 34 & 34 & 36 & 35 & 37 & 42 & 42 & 43 & 43 \\
\hline 1096 & 32 & 34 & 34 & 36 & 35 & 36 & 42 & 42 & 43 & 43 \\
\hline 1099 & 32 & 34 & 34 & 35 & 36 & 35 & 44 & 42 & 46 & 43 \\
\hline 1102 & 35 & 31 & 36 & 32 & 37 & 33 & 44 & 42 & 44 & 42 \\
\hline 1105 & 32 & 35 & 33 & 36 & 34 & 36 & 41 & 42 & 44 & 44 \\
\hline 1108 & 32 & 34 & 33 & 35 & 34 & 35 & 42 & 42 & 45 & 43 \\
\hline 1111 & 32 & 35 & 33 & 35 & 36 & 35 & 43 & 42 & 47 & 43 \\
\hline 1114 & 31 & 36 & 33 & 37 & 34 & 37 & 45 & 43 & 49 & 46 \\
\hline 1117 & 32 & 35 & 33 & 36 & 35 & 38 & 45 & 44 & 46 & 46 \\
\hline 1119 & 31 & 35 & 33 & 37 & 35 & 38 & 47 & 44 & 47 & 46 \\
\hline 1122 & 32 & 35 & 34 & 36 & 36 & 36 & 45 & 43 & 46 & 44 \\
\hline 1125 & 35 & 32 & 36 & 33 & 38 & 34 & 45 & 43 & 46 & 44 \\
\hline 1128 & 32 & 35 & 34 & 38 & 32 & 37 & 44 & 44 & 46 & 47 \\
\hline 1131 & 32 & 35 & 34 & 37 & 35 & 35 & 47 & 44 & 48 & 45 \\
\hline 1134 & 35 & 32 & 37 & 34 & 38 & 33 & 47 & 44 & 47 & 45 \\
\hline 1137 & 32 & 35 & 34 & 37 & 34 & 37 & 45 & 45 & 45 & 46 \\
\hline 1140 & 36 & 31 & 38 & 33 & 38 & 33 & 46 & 43 & 44 & 44 \\
\hline 1142 & 35 & 31 & 37 & 34 & 38 & 34 & 45 & 44 & 45 & 45 \\
\hline 1145 & 36 & 31 & 37 & 33 & 38 & 33 & 44 & 43 & 47 & 44 \\
\hline 1148 & 36 & 31 & 37 & 33 & 36 & 32 & 44 & 43 & 44 & 44 \\
\hline 1151 & 36 & 32 & 36 & 34 & 35 & 33 & 42 & 43 & 43 & 46 \\
\hline 1154 & 35 & 33 & 36 & 34 & 35 & 32 & 44 & 43 & 46 & 44 \\
\hline 1157 & 35 & 32 & 36 & 33 & 35 & 31 & 44 & 43 & 48 & 44 \\
\hline 1160 & 33 & 35 & 33 & 37 & 35 & 36 & 43 & 44 & 45 & 46 \\
\hline
\end{tabular}


CLOSED DOOR SHIELDED FIRE TEST

THERMOCOUPLES AND DISKS AT SPRINKLER AND DETECTOR LOCATIONS - ${ }^{\circ} \mathrm{C}$

\begin{tabular}{|c|c|c|c|c|c|c|c|c|c|c|}
\hline TIME & T14 & D15 & $T 16$ & D17 & T18 & D19 & T20 & D21 & T22 & $\mathrm{D} 23$ \\
\hline 1163 & 31 & 36 & 33 & 37 & 34 & 36 & 46 & 44 & 49 & 47 \\
\hline 1165 & 33 & 33 & 36 & 35 & 38 & 34 & 47 & 44 & 50 & 46 \\
\hline 1168 & 32 & 35 & 34 & 37 & 34 & 37 & 45 & 45 & 48 & 47 \\
\hline 1171 & 32 & 35 & 34 & 36 & 36 & 35 & 46 & 44 & 47 & 45 \\
\hline 1174 & 32 & 36 & 33 & 37 & 33 & 37 & 43 & 45 & 47 & 48 \\
\hline 1177 & 36 & 32 & 37 & 33 & 40 & 34 & 50 & 45 & 51 & 46 \\
\hline 1180 & 35 & 32 & 37 & 34 & 38 & 35 & 50 & 45 & 49 & 46 \\
\hline 1183 & 35 & 32 & 37 & 34 & 35 & 33 & 46 & 45 & 46 & 46 \\
\hline 1186 & 36 & 32 & 36 & 34 & 35 & 33 & 44 & 44 & 46 & 46 \\
\hline 1188 & 33 & 34 & 35 & 35 & 37 & 34 & 43 & 44 & 45 & 46 \\
\hline 1191 & 34 & 34 & 35 & 36 & 35 & 36 & 46 & 45 & 44 & 47 \\
\hline 1194 & 36 & 32 & 38 & 33 & 39 & 33 & 46 & 44 & 46 & 45 \\
\hline 1197 & 32 & 36 & 34 & 37 & 35 & 37 & 47 & 45 & 47 & 47 \\
\hline 1200 & 33 & 35 & 34 & 37 & 34 & 37 & 48 & 46 & 49 & 48 \\
\hline 1203 & 33 & 35 & 35 & 37 & 35 & 35 & 46 & 45 & 46 & 47 \\
\hline 1206 & 34 & 33 & 35 & 34 & 37 & 34 & 44 & 44 & 45 & 45 \\
\hline 1208 & 32 & 36 & 33 & 37 & 33 & 37 & 44 & 45 & 43 & 47 \\
\hline 1211 & 32 & 36 & 34 & 37 & 35 & 37 & 47 & 46 & 46 & 47 \\
\hline 1214 & 32 & 36 & 35 & 38 & 35 & 37 & 47 & 46 & 45 & 47 \\
\hline 1217 & 36 & 32 & 38 & 34 & 39 & 34 & 47 & 45 & 45 & 45 \\
\hline 1220 & 36 & 32 & 38 & 33 & 38 & 34 & 46 & 45 & 46 & 45 \\
\hline 1223 & 37 & 32 & 37 & 34 & 39 & 34 & 44 & 44 & 45 & 45 \\
\hline 1226 & 36 & 33 & 37 & 34 & 38 & 34 & 43 & 44 & 44 & 44 \\
\hline 1229 & 36 & 32 & 38 & 35 & 38 & 34 & 44 & 44 & 48 & 46 \\
\hline 1232 & 36 & 32 & 38 & 34 & 39 & 34 & 45 & 44 & 49 & 45 \\
\hline
\end{tabular}


CLOSED DOOR SHIELDED FIRE TEST

THERMOCOUPLES AND DISKS AT SPRINKLER AND DETECTOR LOCATIONS - ${ }^{\circ} \mathrm{C}$

\begin{tabular}{|c|c|c|c|c|c|c|c|c|c|c|}
\hline TIME & T14 & D15 & T16 & D17 & T18 & D19 & T20 & D21 & T22 & D23 \\
\hline 1234 & 33 & 35 & 36 & 37 & 36 & 35 & 44 & 44 & 46 & 46 \\
\hline 1237 & 33 & 35 & 34 & 37 & 35 & 36 & 46 & 45 & 46 & 46 \\
\hline 1240 & 33 & 34 & 36 & 36 & 36 & 35 & 46 & 44 & 46 & 46 \\
\hline 1243 & 34 & 34 & 37 & 35 & 38 & 35 & 48 & 45 & 46 & 45 \\
\hline 1246 & 32 & 36 & 33 & 38 & 33 & 37 & 43 & 45 & 44 & 47 \\
\hline 1249 & 33 & 36 & 34 & 37 & 36 & 36 & 45 & 45 & 45 & 46 \\
\hline 1252 & 33 & 35 & 34 & 36 & 37 & 35 & 45 & 44 & 48 & 45 \\
\hline 1255 & 33 & 35 & 34 & 36 & 36 & 37 & 45 & 45 & 46 & 47 \\
\hline 1257 & 33 & 35 & 34 & 37 & 36 & 37 & 44 & 45 & 45 & 47 \\
\hline 1260 & 33 & 36 & 34 & 37 & 36 & 38 & 46 & 45 & 47 & 47 \\
\hline 1263 & 36 & 32 & 37 & 33 & 40 & 35 & 47 & 44 & 53 & 47 \\
\hline 1266 & 36 & 32 & 38 & 34 & 40 & 35 & 48 & 45 & 51 & 47 \\
\hline 1269 & 36 & 32 & 39 & 34 & 39 & 35 & 50 & 45 & 49 & 47 \\
\hline 1272 & 35 & 32 & 37 & 34 & 38 & 35 & 48 & 45 & 47 & 46 \\
\hline 1275 & 32 & 36 & 35 & 38 & 37 & 38 & 46 & 46 & 45 & 48 \\
\hline 1278 & 33 & 35 & 34 & 37 & 34 & 38 & 44 & 46 & 46 & 48 \\
\hline 1280 & 36 & 32 & 37 & 34 & 37 & 33 & 46 & 45 & 50 & 46 \\
\hline 1283 & 32 & 36 & 34 & 38 & 35 & 37 & 43 & 46 & 47 & 48 \\
\hline 1286 & 32 & 36 & 34 & 37 & 36 & 38 & 46 & 46 & 48 & 48 \\
\hline 1289 & 32 & 36 & 34 & 37 & 36 & 38 & 48 & 46 & 49 & 48 \\
\hline 1292 & 32 & 37 & 34 & 39 & 34 & 38 & 47 & 46 & 49 & 49 \\
\hline 1295 & 36 & 32 & 39 & 34 & 36 & 33 & 50 & 46 & 51 & 48 \\
\hline 1298 & 36 & 32 & 38 & 34 & 39 & 34 & 49 & 46 & 49 & 47 \\
\hline 1301 & 32 & 36 & 34 & 39 & 35 & 39 & 47 & 47 & 47 & 49 \\
\hline 1303 & 36 & 32 & 38 & 34 & 39 & 35 & 48 & 46 & 47 & 47 \\
\hline
\end{tabular}


CLOSED DOOR SHIELDED FIRE TEST

THERMOCOUPLES AND DISKS AT SPRINKLER AND DETECTOR LOCATIONS - ${ }^{\circ} \mathrm{C}$

\begin{tabular}{llllllllllll}
\hline TIME & T14 & D15 & T16 & D17 & T18 & D19 & T20 & D21 & T22 & D23 \\
\hline 1306 & 36 & 33 & 38 & 34 & 38 & 34 & 48 & 46 & 49 & 47
\end{tabular}


CLOSED DOOR SHIELDED FIRE TEST

THERMOCOUPLES AND DISKS AT SPRINKLER AND DETECTOR LOCATION - ${ }^{\circ} \mathrm{C}$

\begin{tabular}{|c|c|c|c|c|c|c|c|c|c|c|}
\hline TIME & T24 & D25 & T26 & $\mathrm{D} 27$ & T28 & D29 & PAN A & PAN B & PAN C & PAN D \\
\hline .53 & 29 & 27 & 28 & 27 & - & $\cdots$ & 28 & 29 & 28 & 28 \\
\hline-49 & 26 & 28 & 27 & 28 & - & $\cdots$ & 28 & 24 & 28 & 27 \\
\hline-46 & 26 & 28 & 27 & 28 & - & - & 28 & 24 & 29 & 27 \\
\hline-43 & 29 & 27 & 29 & 27 & $\cdots$ & -- & 28 & 29 & 28 & 28 \\
\hline-40 & 27 & 28 & 27 & 28 & -- & -- & 27 & 24 & 30 & 27 \\
\hline-37 & 29 & 27 & 29 & 27 & $-\ldots$ & $\cdots$ & 28 & 29 & 27 & 28 \\
\hline-35 & 26 & 28 & 27 & 28 & -- & $\ldots$ & 28 & 24 & 29 & 27 \\
\hline-32 & 28 & 28 & 27 & 28 & $\cdots$ & - & 27 & 24 & 30 & 28 \\
\hline-29 & 26 & 28 & 27 & 28 & - & $\ldots$ & 27 & 24 & 29 & 27 \\
\hline-26 & 27 & 28 & 27 & 28 & $-\infty$ & - & 27 & 24 & 30 & 28 \\
\hline-23 & 29 & 27 & 28 & 27 & - & - & 27 & 28 & 29 & 28 \\
\hline-20 & 26 & 28 & 27 & 28 & $\cdots$ & - & 28 & 24 & 29 & 27 \\
\hline-17 & 29 & 28 & 28 & 27 & $\ldots$ & - & 27 & 26 & 29 & 28 \\
\hline-15 & 29 & 27 & 28 & 27 & --. & $\cdots$ & 28 & 28 & 28 & 28 \\
\hline-12 & 29 & 27 & 29 & 27 & - & - & 28 & 29 & 27 & 29 \\
\hline-9 & 29 & 28 & 28 & 27 & - & $\longrightarrow$ & 27 & 26 & 29 & 28 \\
\hline-6 & 29 & 27 & 28 & 27 & - & - & 28 & 28 & 28 & 29 \\
\hline-3 & 29 & 27 & 29 & 27 & $\cdots$ & - & 29 & 29 & 27 & 28 \\
\hline .0 & 29 & 27 & 28 & 27 & - & - & 27 & 27 & 29 & 28 \\
\hline 1 & 30 & 27 & 29 & 27 & - & - & 28 & 29 & 27 & 29 \\
\hline 4 & 29 & 27 & 29 & 27 & - & - & 29 & 29 & 27 & 29 \\
\hline 7 & 27 & 28 & 28 & 28 & $\ldots$ & $\ldots$ & 28 & 25 & 28 & 28 \\
\hline 10 & 29 & 27 & 28 & 27 & - & - & 29 & 29 & 27 & 29 \\
\hline 13 & 29 & 27 & 28 & 27 & - & $-\infty$ & 28 & 29 & 28 & 29 \\
\hline 16 & 26 & 29 & 27 & 29 & - & - & 28 & 23 & 29 & 28 \\
\hline
\end{tabular}


THERMOCOUPLES AND DISKS AT SPRINKLER AND DETECTOR LOCATION - ${ }^{\circ} \mathrm{C}$

\begin{tabular}{|c|c|c|c|c|c|c|c|c|c|c|}
\hline TIME & $T 24$ & $\mathrm{D} 25$ & T26 & $\mathrm{D} 27$ & T28 & D29 & PAN A & PAN B & PAN C & PAN D \\
\hline 19 & 29 & 28 & 28 & 27 & - & - & 30 & 28 & 27 & 28 \\
\hline 22 & 27 & 28 & 28 & 28 & - & - & 29 & 26 & 27 & 28 \\
\hline 24 & 27 & 29 & 27 & 29 & - & - & 29 & 24 & 29 & 29 \\
\hline 27 & 29 & 27 & 28 & 27 & - & - & 31 & 29 & 27 & 29 \\
\hline 30 & 26 & 29 & 27 & 29 & - & - & 29 & 24 & 29 & 29 \\
\hline 33 & 29 & 28 & 28 & 28 & - & - & 30 & 25 & 29 & 30 \\
\hline 36 & 30 & 27 & 29 & 27 & - & - & 31 & 30 & 27 & 31 \\
\hline 39 & 27 & 29 & 28 & 29 & - & - & 30 & 25 & 29 & 30 \\
\hline 41 & 30 & 28 & 29 & 28 & - & - & 31 & 29 & 29 & 32 \\
\hline 44 & 28 & 28 & 29 & 29 & - & - & 31 & 27 & 27 & 31 \\
\hline 47 & 30 & 27 & 30 & 27 & - & - & 32 & 31 & 28 & 32 \\
\hline 50 & 30 & 28 & 30 & 28 & - & - & 32 & 30 & 29 & 32 \\
\hline 53 & 29 & 28 & 30 & 28 & - & - & 33 & 31 & 28 & 32 \\
\hline 56 & 30 & 27 & 30 & 28 & - & - & 33 & 31 & 28 & 32 \\
\hline 59 & 28 & 28 & 30 & 29 & - & - & 32 & 29 & 28 & 32 \\
\hline 61 & 29 & 28 & 31 & 28 & - & - & 33 & 31 & 28 & 32 \\
\hline 64 & 28 & 29 & 29 & 29 & - & - & 32 & 26 & 30 & 32 \\
\hline 67 & 31 & 27 & 31 & 28 & - & - & 33 & 32 & 28 & 34 \\
\hline 70 & 28 & 29 & 29 & 30 & - & - & 32 & 27 & 30 & 32 \\
\hline 73 & 31 & 29 & 30 & 29 & - & - & 33 & 29 & 30 & 33 \\
\hline 76 & 30 & 29 & 30 & 30 & - & - & 33 & 27 & 30 & 33 \\
\hline 79 & 32 & 29 & 31 & 29 & - & - & 35 & 31 & 28 & 33 \\
\hline 82 & 30 & 29 & 30 & 30 & - & - & 33 & 27 & 31 & 33 \\
\hline 84 & 31 & 29 & 31 & 30 & - & - & 34 & 27 & 31 & 34 \\
\hline 87 & 32 & 29 & 33 & 29 & - & - & 34 & 31 & 31 & 35 \\
\hline
\end{tabular}


CLOSED DOOR SHIELDED FIRE TEST

THERMOCOUPLES AND DISKS AT SPRINKLER AND DETECTOR LOCATION - ${ }^{\circ} \mathrm{C}$

\begin{tabular}{|c|c|c|c|c|c|c|c|c|c|c|}
\hline TIME & T24 & D25 & T26 & $\mathrm{D} 27$ & T28 & D29 & PAN A & PAN B & PAN C & PAN D \\
\hline 90 & 31 & 30 & 32 & 30 & - & - & 34 & 28 & 31 & 34 \\
\hline 93 & 33 & 29 & 33 & 29 & - & $\cdots$ & 35 & 31 & 29 & 35 \\
\hline 96 & 31 & 30 & 32 & 31 & - & - & 34 & 27 & 31 & 34 \\
\hline 99 & 34 & 29 & 33 & 30 & - & - & 36 & 31 & 29 & 35 \\
\hline 101 & 34 & 29 & 34 & 30 & - & - & 36 & 34 & 29 & 36 \\
\hline 104 & 34 & 29 & 35 & 30 & - & - & 37 & 34 & 29 & 36 \\
\hline 107 & 34 & 30 & 34 & 31 & - & - & 35 & 31 & 31 & 37 \\
\hline 110 & 35 & 30 & 35 & 30 & - & - & 37 & 33 & 29 & 37 \\
\hline 113 & 33 & 31 & 34 & 32 & - & $\longrightarrow$ & 36 & 28 & 30 & 35 \\
\hline 116 & 32 & 32 & 34 & 33 & - & - & 36 & 28 & 31 & 36 \\
\hline 118 & 36 & 30 & 36 & 31 & - & - & 38 & 33 & 30 & 38 \\
\hline 121 & 35 & 31 & 35 & 32 & $\cdots$ & - & 38 & 33 & 30 & 37 \\
\hline 124 & 33 & 32 & 35 & 33 & - & - & 37 & 30 & 31 & 37 \\
\hline 127 & 33 & 32 & 35 & 33 & - & - & 38 & 30 & 30 & 38 \\
\hline 130 & 33 & 32 & 35 & 33 & - & - & 38 & 31 & 32 & 38 \\
\hline 133 & 34 & 33 & 36 & 34 & - & - & 37 & 30 & 32 & 38 \\
\hline 136 & 37 & 31 & 38 & 32 & - & - & 41 & 35 & 31 & 40 \\
\hline 138 & 34 & 33 & 36 & 34 & - & - & 38 & 30 & 33 & 38 \\
\hline 141 & 38 & 31 & 38 & 32 & - & - & 40 & 36 & 31 & 41 \\
\hline 144 & 38 & 32 & 38 & 33 & - & - & 40 & 34 & 31 & 40 \\
\hline 147 & 38 & 32 & 38 & 33 & - & - & 41 & 34 & 31 & 40 \\
\hline 150 & 36 & 33 & 37 & 35 & - & - & 40 & 31 & 34 & 41 \\
\hline 153 & 38 & 33 & 38 & 34 & - & - & 40 & 33 & 34 & 42 \\
\hline 156 & 38 & 32 & 39 & 34 & - & - & 40 & 36 & 33 & 42 \\
\hline 158 & 38 & 33 & 39 & 34 & - & - & 41 & 35 & 33 & 42 \\
\hline
\end{tabular}


THERMOCOUPLES AND DISKS AT SPRINKLER AND DETECTOR LOCATION - ${ }^{\circ} \mathrm{C}$

\begin{tabular}{|c|c|c|c|c|c|c|c|c|c|c|}
\hline TME & T24 & D25 & T26 & D27 & T28 & 029 & PAN A & PAN B & PAN C & PAN D \\
\hline 161 & 38 & 33 & 38 & 35 & - & - & 40 & 33 & 34 & 41 \\
\hline 164 & 38 & 34 & 40 & 35 & - & - & 40 & 34 & 35 & 42 \\
\hline 167 & 40 & 33 & 41 & 35 & - & - & 42 & 36 & 33 & 43 \\
\hline 170 & 39 & 34 & 40 & 35 & - & - & 43 & 37 & 32 & 42 \\
\hline 173 & 40 & 34 & 40 & 35 & - & - & 44 & 37 & 33 & 42 \\
\hline 176 & 38 & 35 & 39 & 37 & - & - & 44 & 36 & 33 & 42 \\
\hline 179 & 37 & 36 & 39 & 37 & - & - & 42 & 32 & 35 & 43 \\
\hline 181 & 38 & 35 & 41 & 37 & - & - & 44 & 37 & 33 & 44 \\
\hline 184 & 39 & 35 & 41 & 37 & - & - & 44 & 37 & 34 & 43 \\
\hline 187 & 39 & 36 & 41 & 38 & - & - & 44 & 35 & 35 & 43 \\
\hline 190 & 40 & 37 & 41 & 38 & - & - & 44 & 33 & 35 & 44 \\
\hline 193 & 43 & 35 & 43 & 37 & $\ldots$ & - & 45 & 37 & 34 & 45 \\
\hline 196 & 40 & 37 & 43 & 39 & - & - & 45 & 33 & 35 & 44 \\
\hline 199 & 43 & 36 & 44 & 37 & - & - & 46 & 38 & 34 & 45 \\
\hline 201 & 41 & 38 & 43 & 39 & - & - & 43 & 32 & 36 & 45 \\
\hline 204 & 44 & 37 & 43 & 38 & - & - & 46 & 37 & 34 & 46 \\
\hline 207 & 40 & 39 & 42 & 40 & - & - & 45 & 32 & 36 & 45 \\
\hline 210 & 41 & 38 & 42 & 40 & - & - & 44 & 34 & 36 & 45 \\
\hline 213 & 41 & 38 & 43 & 40 & - & - & 47 & 36 & 35 & 46 \\
\hline 216 & 43 & 38 & 43 & 40 & - & - & 47 & 38 & 37 & 46 \\
\hline 219 & 43 & 38 & 45 & 40 & - & - & 47 & 39 & 37 & 48 \\
\hline 221 & 45 & 38 & 46 & 39 & - & - & 48 & 40 & 36 & 48 \\
\hline 224 & 44 & 38 & 46 & 40 & - & - & 48 & 40 & 37 & 48 \\
\hline 227 & 41 & 40 & 45 & 42 & - & - & 48 & 35 & 38 & 46 \\
\hline 230 & 45 & 39 & 46 & 40 & - & - & 50 & 39 & 36 & 48 \\
\hline
\end{tabular}


CLOSED DOOR SHIELDED FIRE TEST

THERMOCOUPLES AND DISKS AT SPRINKLER AND DETECTOR LOCATION - ${ }^{\circ} \mathrm{C}$

\begin{tabular}{|c|c|c|c|c|c|c|c|c|c|c|}
\hline TME & T24 & D25 & T26 & $\mathrm{D} 27$ & T28 & D29 & PAN A & PAN B & PAN C & PAN D \\
\hline 233 & 45 & 39 & 45 & 41 & - & - & 49 & 38 & 38 & 49 \\
\hline 236 & 45 & 40 & 47 & 42 & - & - & 49 & 36 & 38 & 49 \\
\hline 238 & 46 & 40 & 47 & 41 & - & $\ldots$ & 51 & 40 & 38 & 50 \\
\hline 241 & 46 & 39 & 48 & 41 & - & $\cdots$ & 52 & 41 & 37 & 51 \\
\hline 244 & 43 & 41 & 46 & 43 & - & $\cdots$ & 51 & 35 & 38 & 49 \\
\hline 247 & 43 & 41 & 47 & 43 & - & - & 51 & 35 & 39 & 48 \\
\hline 250 & 45 & 41 & 49 & 42 & - & - & 51 & 41 & 37 & 49 \\
\hline 253 & 46 & 40 & 48 & 42 & - & $\ldots$ & 51 & 40 & 37 & 51 \\
\hline 256 & 44 & 42 & 47 & 44 & - & $-\cdots$ & 51 & 35 & 39 & 49 \\
\hline 258 & 47 & 42 & 48 & 43 & - & $\longrightarrow$ & 52 & 41 & 38 & 53 \\
\hline 261 & 49 & 41 & 48 & 43 & - & $\cdots$ & 51 & 41 & 39 & 52 \\
\hline 264 & 50 & 41 & 50 & 43 & $\omega$ & - & 52 & 43 & 38 & 53 \\
\hline 267 & 49 & 42 & 51 & 44 & - & - & 51 & 43 & 38 & 52 \\
\hline 270 & 47 & 43 & 51 & 46 & - & - & 51 & 39 & 40 & 51 \\
\hline 273 & 50 & 42 & 52 & 44 & - & $\longrightarrow$ & 53 & 43 & 38 & 53 \\
\hline 275 & 50 & 43 & 53 & 45 & - & - & 55 & 44 & 39 & 52 \\
\hline 278 & 49 & 44 & 51 & 47 & $\longrightarrow$ & $\cdots$ & 54 & 41 & 41 & 53 \\
\hline 281 & 50 & 45 & 53 & 47 & - & - & 54 & 41 & 39 & 54 \\
\hline 284 & 52 & 44 & 55 & 46 & - & - & 55 & 46 & 39 & 58 \\
\hline 287 & 51 & 46 & 53 & 48 & - & - & 53 & 41 & 42 & 55 \\
\hline 290 & 52 & 46 & 54 & 49 & - & - & 56 & 41 & 42 & 56 \\
\hline 293 & 53 & 45 & 56 & 48 & - & - & 56 & 45 & 43 & 57 \\
\hline 295 & 53 & 47 & 55 & 50 & - & - & 57 & 42 & 44 & 58 \\
\hline 298 & 53 & 47 & 58 & 50 & - & - & 57 & 44 & 44 & 58 \\
\hline 301 & 54 & 48 & 58 & 51 & $\ldots$ & $\ldots$ & 56 & 41 & 46 & 58 \\
\hline
\end{tabular}


CLOSED DOOR SHIELDED FIRE TEST

THERMOCOUPLES AND DISKS AT SPRINKLER AND DETECTOR LOCATION - ${ }^{\circ} \mathrm{C}$

\begin{tabular}{|c|c|c|c|c|c|c|c|c|c|c|}
\hline TIME & T24 & $\mathrm{D} 25$ & T26 & $\mathrm{D} 27$ & T28 & 029 & PAN A & PAN B & PAN C & PAN D \\
\hline 304 & 56 & 47 & 58 & 50 & - & - & 57 & 46 & 45 & 61 \\
\hline 307 & 59 & 47 & 60 & 50 & - & - & 59 & 49 & 44 & 62 \\
\hline 310 & 56 & 49 & 60 & 52 & - & - & 59 & 46 & 46 & 63 \\
\hline 313 & 57 & 50 & 60 & 53 & - & - & 61 & 44 & 46 & 61 \\
\hline 315 & 60 & 49 & 61 & 51 & - & - & 62 & 49 & 45 & 64 \\
\hline 318 & 56 & 51 & 60 & 54 & - & - & 63 & 44 & 47 & 62 \\
\hline 321 & 55 & 51 & 60 & 54 & - & - & 63 & 45 & 47 & 63 \\
\hline 324 & 60 & 50 & 61 & 53 & - & - & 66 & 50 & 45 & 65 \\
\hline 327 & 61 & 51 & 63 & 54 & - & - & 65 & 50 & 45 & 66 \\
\hline 330 & 61 & 53 & 62 & 55 & - & - & 67 & 45 & 45 & 64 \\
\hline 332 & 64 & 52 & 65 & 54 & - & - & 68 & 52 & 47 & 66 \\
\hline 335 & 60 & 53 & 66 & 57 & - & - & 69 & 47 & 48 & 66 \\
\hline 338 & 65 & 52 & 67 & 56 & - & -.- & 68 & 52 & 48 & 69 \\
\hline 341 & 62 & 55 & 66 & 58 & - & - & 67 & 46 & 50 & 66 \\
\hline 344 & 62 & 55 & 66 & 59 & - & - & 67 & 47 & 50 & 68 \\
\hline 347 & 65 & 55 & 68 & 59 & - & - & 69 & 51 & 49 & 68 \\
\hline 350 & 67 & 56 & 69 & 59 & - & - & 70 & 51 & 50 & 70 \\
\hline 353 & 65 & 57 & 69 & 61 & - & - & 69 & 49 & 51 & 70 \\
\hline 355 & 70 & 57 & 70 & 60 & - & - & 69 & 56 & 51 & 73 \\
\hline 358 & 72 & 58 & 70 & 61 & - & - & 71 & 53 & 52 & 72 \\
\hline 361 & 71 & 58 & 72 & 61 & - & - & 75 & 58 & 50 & 73 \\
\hline 364 & 71 & 60 & 71 & 62 & - & - & 73 & 55 & 53 & 74 \\
\hline 367 & 73 & 60 & 74 & 63 & - & - & 73 & 57 & 53 & 76 \\
\hline 370 & 70 & 62 & 73 & 65 & - & - & 72 & 53 & 54 & 74 \\
\hline 372 & 72 & 61 & 75 & 64 & - & - & 74 & 58 & 55 & $\pi$ \\
\hline
\end{tabular}


CLOSED DOOR SHIELDED FIRE TEST

THERMOCOUPLES AND DISKS AT SPRINKLER AND DETECTOR LOCATION - ${ }^{\circ} \mathrm{C}$

\begin{tabular}{|c|c|c|c|c|c|c|c|c|c|c|}
\hline TIME & T24 & D25 & T26 & D27 & T28 & D29 & PAN A & PAN B & PAN C & PAN D \\
\hline 375 & 71 & 62 & 76 & 66 & $\cdots$ & - & 75 & 56 & 55 & 77 \\
\hline 378 & 69 & 62 & 74 & 66 & $\cdots$ & - & 75 & 55 & 55 & 78 \\
\hline 381 & 73 & 62 & 77 & 65 & - & - & 76 & 60 & 53 & 80 \\
\hline 384 & 74 & 63 & 79 & 67 & - & -- & 78 & 64 & 53 & 77 \\
\hline 387 & 74 & 63 & 79 & 67 & - & - & 79 & 62 & 54 & 77 \\
\hline 390 & 77 & 63 & 79 & 68 & -- & - & 79 & 62 & 56 & 80 \\
\hline 392 & 73 & 64 & 78 & 69 & - & - & 76 & 57 & 56 & 80 \\
\hline 395 & 75 & 64 & 76 & 68 & - & - & 78 & 62 & 58 & 77 \\
\hline 398 & 75 & 66 & 78 & 70 & - & - & 80 & 62 & 58 & 78 \\
\hline 401 & 73 & 66 & 78 & 70 & - & - & 79 & 61 & 59 & 78 \\
\hline 404 & 75 & 67 & 78 & 71 & - & - & 79 & 60 & 59 & 78 \\
\hline 407 & 76 & 67 & 79 & 71 & - & - & 77 & 58 & 58 & 82 \\
\hline 409 & 75 & 67 & 78 & 72 & - & - & 78 & 60 & 59 & 79 \\
\hline 412 & 78 & 66 & 79 & 70 & $\cdots$ & - & 79 & 67 & 57 & 80 \\
\hline 415 & 74 & 68 & 78 & 72 & - & - & 77 & 62 & 60 & 78 \\
\hline 418 & 74 & 67 & 78 & 72 & - & - & 76 & 60 & 59 & 79 \\
\hline 421 & 74 & 67 & 78 & 71 & - & - & 77 & 62 & 57 & 79 \\
\hline 424 & 73 & 67 & 78 & 71 & - & - & 78 & 62 & 59 & 78 \\
\hline 427 & 72 & 68 & 76 & 73 & - & - & 77 & 60 & 58 & 79 \\
\hline 429 & 75 & 67 & 76 & 71 & - & - & 79 & 68 & 56 & 80 \\
\hline 432 & 73 & 69 & 77 & 73 & - & - & 80 & 62 & 57 & 80 \\
\hline 435 & 73 & 69 & 79 & 73 & - & - & 80 & 63 & 57 & 81 \\
\hline 438 & 75 & 68 & 79 & 72 & - & - & 81 & 66 & 57 & 82 \\
\hline 441 & 76 & 68 & 79 & 72 & - & - & 83 & 66 & 57 & 81 \\
\hline 444 & 73 & 70 & 77 & 73 & - & - & 81 & 63 & 58 & 80 \\
\hline
\end{tabular}


CLOSED DOOR SHIELDED FIRE TEST

THERMOCOUPLES AND DISKS AT SPRINKLER AND DETECTOR LOCATION - ${ }^{\circ} \mathrm{C}$

\begin{tabular}{|c|c|c|c|c|c|c|c|c|c|c|}
\hline TIME & T24 & D25 & T26 & $\mathrm{D} 27$ & T28 & $\mathrm{D} 29$ & PAN A & PAN B & PAN C & PAN D \\
\hline 447 & 75 & 70 & $\pi$ & 73 & - & - & 82 & 62 & 58 & 80 \\
\hline 449 & 73 & 70 & 76 & 73 & - & - & 81 & 61 & 59 & 82 \\
\hline 452 & 74 & 70 & 76 & 73 & - & - & 82 & 64 & 60 & 84 \\
\hline 455 & 74 & 70 & 78 & 74 & - & - & 83 & 62 & 60 & 84 \\
\hline 458 & 76 & 69 & 80 & 72 & - & $-\infty$ & 83 & 69 & 60 & 84 \\
\hline 461 & 74 & 70 & 80 & 74 & - & - & 82 & 65 & 62 & 84 \\
\hline 464 & 73 & 70 & 78 & 74 & - & - & 83 & 64 & 60 & 84 \\
\hline 466 & 75 & 70 & 81 & 74 & - & - & 84 & 65 & 61 & 86 \\
\hline 469 & 78 & 70 & 79 & 73 & - & - & 84 & 64 & 62 & 86 \\
\hline 472 & 78 & 70 & 81 & 73 & - & - & 84 & 65 & 60 & 88 \\
\hline 475 & 77 & 70 & 81 & 74 & - & - & 86 & 63 & 62 & 87 \\
\hline 478 & $\pi$ & 70 & 83 & 74 & - & - & 86 & 66 & 61 & 87 \\
\hline 481 & 78 & 71 & 81 & 74 & - & - & 87 & 66 & 62 & 88 \\
\hline 484 & 76 & 72 & 83 & 76 & - & - & 87 & 64 & 64 & 87 \\
\hline 486 & 76 & 71 & 84 & 75 & - & - & 89 & 71 & 62 & 86 \\
\hline 489 & 78 & 71 & 83 & 75 & - & - & 91 & 73 & 61 & 90 \\
\hline 492 & 78 & 73 & 82 & $\pi$ & - & - & 88 & 67 & 62 & 87 \\
\hline 495 & 80 & 71 & 85 & 76 & - & - & 90 & 70 & 62 & 90 \\
\hline 498 & 78 & 73 & 84 & $\pi$ & - & - & 89 & 64 & 64 & 90 \\
\hline 501 & 82 & 72 & 85 & 76 & - & - & 89 & 70 & 64 & 93 \\
\hline 504 & 79 & 74 & 86 & 78 & - & - & 91 & 66 & 65 & 91 \\
\hline 506 & 84 & 73 & 85 & 78 & - & - & 90 & 70 & 64 & 93 \\
\hline 509 & 80 & 74 & 86 & 78 & - & - & 92 & 70 & 67 & 92 \\
\hline 512 & 81 & 73 & 88 & 78 & - & - & 97 & 73 & 64 & 94 \\
\hline 515 & 83 & 75 & 86 & 80 & - & - & 95 & 67 & 67 & 93 \\
\hline
\end{tabular}


CLOSED DOOR SHIELDED FIRE TEST

THERMOCOUPLES AND DISKS AT SPRINKLER AND DETECTOR LOCATION - ${ }^{\circ} \mathrm{C}$

\begin{tabular}{|c|c|c|c|c|c|c|c|c|c|c|}
\hline TIME & $T 24$ & D25 & T26 & $\mathrm{D} 27$ & T28 & $\mathrm{D} 29$ & PAN A & PAN $B$ & PAN C & PAN D \\
\hline 518 & 82 & 76 & 88 & 80 & - & $\ldots$ & 97 & 67 & 67 & 94 \\
\hline 521 & 86 & 75 & 88 & 79 & - & $\ldots$ & 99 & 74 & 64 & 96 \\
\hline 523 & 88 & 75 & 88 & 79 & - & - & 99 & 74 & 67 & 96 \\
\hline 526 & 85 & 76 & 91 & 80 & - & - & 101 & 73 & 68 & 95 \\
\hline 529 & 88 & 76 & 94 & 80 & $\cdots$ & $\ldots$ & 103 & 76 & 68 & 97 \\
\hline 532 & 88 & $\pi$ & 93 & 82 & - & - & 101 & 74 & 69 & 99 \\
\hline 535 & 84 & 78 & 93 & 84 & - & -.. & 103 & 74 & 70 & 96 \\
\hline 538 & 89 & $\pi$ & 95 & 83 & - & - & 106 & 77 & 69 & 102 \\
\hline 540 & 91 & 78 & 95 & 83 & - & - & 107 & 76 & 69 & 101 \\
\hline 543 & 88 & 79 & 94 & 85 & - & - & 108 & 71 & 70 & 102 \\
\hline 546 & 90 & 80 & 93 & 86 & - & - & 108 & 72 & 71 & 103 \\
\hline 549 & 88 & 81 & 94 & 86 & $-\infty$ & - & 110 & 72 & 73 & 100 \\
\hline 552 & 92 & 80 & 95 & 85 & - & - & 110 & 78 & 71 & 102 \\
\hline 555 & 91 & 82 & 96 & 88 & - & - & 105 & 75 & 72 & 106 \\
\hline 558 & 94 & 83 & 98 & 88 & - & - & 109 & 76 & 73 & 107 \\
\hline 560 & 94 & 82 & 100 & 87 & - & - & 115 & 80 & 72 & 108 \\
\hline 563 & 92 & 83 & 99 & 88 & - & - & 114 & 81 & 73 & 107 \\
\hline 566 & 97 & 83 & 98 & 88 & - & - & 115 & 81 & 73 & 107 \\
\hline 569 & 97 & 84 & 99 & 88 & - & - & 113 & 81 & 75 & 112 \\
\hline 572 & 99 & 86 & 99 & 90 & - & - & 115 & 79 & 74 & 110 \\
\hline 575 & 102 & 85 & 102 & 90 & - & - & 116 & 84 & 75 & 108 \\
\hline 578 & 101 & 86 & 102 & 90 & - & - & 113 & 82 & 78 & 110 \\
\hline 580 & 94 & 87 & 102 & 92 & - & - & 116 & 83 & 79 & 109 \\
\hline 583 & 99 & 88 & 103 & 93 & - & - & 113 & 79 & 78 & 112 \\
\hline 586 & 96 & 88 & 104 & 93 & $\ldots$ & - & 118 & 80 & 76 & 113 \\
\hline
\end{tabular}


THERMOCOUPLES AND DISKS AT SPRINKLER AND DETECTOR LOCATION $-{ }^{\circ} \mathrm{C}$

\begin{tabular}{|c|c|c|c|c|c|c|c|c|c|c|}
\hline TIME & T24 & D25 & T26 & 027 & T28 & D29 & PAN A & PAN B & PAN C & PAN D \\
\hline 589 & 100 & 87 & 105 & 93 & - & - & 117 & 83 & 75 & 116 \\
\hline 592 & 100 & 88 & 106 & 93 & - & - & 119 & 88 & 75 & 113 \\
\hline 595 & 99 & 90 & 106 & 96 & - & - & 118 & 83 & 77 & 112 \\
\hline 597 & 101 & 90 & 109 & 96 & - & - & 122 & 88 & $\pi$ & 113 \\
\hline 600 & 100 & 90 & 110 & 97 & $\cdots$ & - & 122 & 88 & $\pi$ & 117 \\
\hline 603 & 105 & 90 & 109 & 96 & - & - & 126 & 88 & 79 & 114 \\
\hline 606 & 101 & 91 & 109 & 98 & - & - & 126 & 85 & 82 & 116 \\
\hline 609 & 103 & 91 & 109 & 98 & --- & - & 126 & 92 & 80 & 120 \\
\hline 612 & 102 & 92 & 108 & 99 & -- & -- & 126 & 88 & 83 & 119 \\
\hline 615 & 104 & 92 & 110 & 98 & - & -- & 128 & 94 & 82 & 117 \\
\hline 617 & 106 & 94 & 108 & 100 & - & $-\infty$ & 127 & 87 & 84 & 120 \\
\hline 620 & 105 & 93 & 110 & 100 & - & - & 130 & 85 & 85 & 121 \\
\hline 623 & 105 & 93 & 113 & 100 & - & - & 131 & 90 & 80 & 117 \\
\hline 626 & 107 & 95 & 111 & 102 & - & - & 131 & 90 & 83 & 122 \\
\hline 629 & 111 & 95 & 115 & 101 & - & - & 130 & 92 & 84 & 122 \\
\hline 632 & 106 & 97 & 116 & 103 & - & - & 131 & 91 & 85 & 122 \\
\hline 635 & 110 & 96 & 118 & 102 & --. & $-\cdots$ & 136 & 97 & 82 & 122 \\
\hline 637 & 112 & 96 & 117 & 103 & - & -- & 135 & 97 & 84 & 124 \\
\hline 640 & 112 & 99 & 117 & 106 & - & - & 137 & 90 & 88 & 125 \\
\hline 643 & 114 & 98 & 115 & 104 & - & - & 134 & 93 & 87 & 127 \\
\hline 646 & 117 & 101 & 115 & 106 & - & - & 134 & 90 & 89 & 124 \\
\hline 649 & 113 & 102 & 119 & 107 & - & - & 136 & 93 & 88 & 127 \\
\hline 652 & 115 & 101 & 118 & 107 & - & - & 137 & 93 & 83 & 128 \\
\hline 654 & 124 & 102 & 116 & 107 & - & - & 139 & 93 & 88 & 124 \\
\hline 657 & 119 & 102 & 117 & 108 & - & - & 137 & 93 & 89 & 124 \\
\hline
\end{tabular}


CLOSED DOOR SHIELDED FIRE TEST

THERMOCOUPLES AND DISKS AT SPRINKLER AND DETECTOR LOCATION - ${ }^{\circ} \mathrm{C}$

\begin{tabular}{|c|c|c|c|c|c|c|c|c|c|c|}
\hline TIME & T24 & D25 & T26 & D27 & $T 28$ & D29 & PAN A & PAN B & PAN C & PAN D \\
\hline 660 & 120 & 104 & 119 & 109 & - & - & 139 & 93 & 90 & 125 \\
\hline 663 & 119 & 103 & 119 & 108 & - & - & 138 & 98 & 92 & 128 \\
\hline 666 & 115 & 105 & 117 & 110 & - & - & 139 & 94 & 90 & 128 \\
\hline 669 & 117 & 104 & 117 & 109 & - & - & 141 & 94 & 87 & 131 \\
\hline 672 & 115 & 105 & 118 & 109 & - & - & 144 & 96 & 90 & 131 \\
\hline 674 & 120 & 105 & 120 & 109 & - & - & 141 & 101 & 88 & 133 \\
\hline 677 & 117 & 106 & 122 & 111 & - & - & 140 & 97 & 89 & 130 \\
\hline 680 & 118 & 106 & 119 & 110 & - & - & 144 & 101 & 88 & 128 \\
\hline 683 & 117 & 107 & 117 & 111 & - & - & 142 & 97 & 95 & 129 \\
\hline 686 & 119 & 105 & 119 & 110 & - & - & 141 & 101 & 93 & 133 \\
\hline 689 & 118 & 107 & 117 & 111 & - & - & 143 & 98 & 92 & 134 \\
\hline 692 & 117 & 108 & 123 & 112 & - & --. & 143 & 98 & 96 & 133 \\
\hline 694 & 118 & 107 & 123 & 111 & - & - & 142 & 97 & 94 & 131 \\
\hline 697 & 64 & 90 & 110 & 108 & - & - & 66 & 34 & 91 & 94 \\
\hline 700 & 72 & 86 & 95 & 103 & - & - & 53 & 39 & 91 & 103 \\
\hline 703 & 89 & 91 & 99 & 104 & - & - & 50 & 35 & 92 & 105 \\
\hline 706 & 89 & 88 & 96 & 100 & - & - & 54 & 40 & 86 & 105 \\
\hline 709 & $\pi 7$ & 85 & 78 & 95 & - & - & 47 & 40 & 79 & 103 \\
\hline 711 & 72 & 83 & 80 & 95 & - & - & 49 & 33 & 75 & 93 \\
\hline 714 & 75 & $\pi$ & 75 & 88 & - & - & 49 & 38 & 70 & 90 \\
\hline 717 & 55 & $\pi$ & 64 & 86 & - & - & 47 & 34 & 71 & 85 \\
\hline 720 & 55 & 73 & 61 & 79 & - & - & 50 & 35 & 67 & 82 \\
\hline 723 & 53 & 70 & 59 & 75 & - & - & 46 & 33 & 64 & 81 \\
\hline 726 & 53 & 67 & 58 & 72 & - & - & 46 & 33 & 63 & 81 \\
\hline 728 & 51 & 61 & 55 & 67 & - & - & 48 & 34 & 61 & 75 \\
\hline
\end{tabular}


THERMOCOUPLES AND DISKS AT SPRINKLER AND DETECTOR LOCATION $-{ }^{\circ} \mathrm{C}$

\begin{tabular}{|c|c|c|c|c|c|c|c|c|c|c|}
\hline TIME & T24 & D25 & T26 & $\mathrm{D} 27$ & T28 & D29 & PAN A & PAN B & PAN C & PAN D \\
\hline 731 & 49 & 60 & 54 & 66 & - & - & 47 & 33 & 59 & 77 \\
\hline 734 & 54 & 52 & 53 & 58 & - & - & 45 & 38 & 56 & 78 \\
\hline 737 & 50 & 52 & 53 & . 56 & - & - & 51 & 38 & 55 & 75 \\
\hline 740 & 44 & 50 & 48 & 56 & - & - & 52 & 34 & 54 & 75 \\
\hline 743 & 44 & 49 & 47 & 54 & - & - & 45 & 35 & 53 & 70 \\
\hline 746 & 45 & 44 & 48 & 49 & - & - & 46 & 39 & 54 & 70 \\
\hline 748 & 48 & 43 & 50 & 48 & - & - & 49 & 38 & 53 & 70 \\
\hline 751 & 43 & 47 & 46 & 50 & - & - & 52 & 34 & 51 & 64 \\
\hline 754 & 47 & 43 & 48 & 46 & - & - & 47 & 36 & 51 & 66 \\
\hline 757 & 42 & 47 & 43 & 49 & - & - & 46 & 32 & 51 & 68 \\
\hline 760 & 47 & 42 & 47 & 44 & - & - & 42 & 38 & 48 & 66 \\
\hline 763 & 46 & 41 & 46 & 42 & - & - & 42 & 38 & 48 & 63 \\
\hline 766 & 41 & 43 & 41 & 45 & - & - & 43 & 33 & 48 & 64 \\
\hline 768 & 42 & 43 & 43 & 45 & - & - & 44 & 33 & 46 & 63 \\
\hline 771 & 43 & 41 & 43 & 42 & - & - & 43 & 36 & 44 & 62 \\
\hline 774 & 46 & 41 & 43 & 41 & - & - & 40 & 36 & 46 & 60 \\
\hline 777 & 40 & 44 & 40 & 44 & - & - & 41 & 32 & 45 & 58 \\
\hline 780 & 43 & 40 & 42 & 40 & - & - & 42 & 36 & 44 & 58 \\
\hline 783 & 39 & 42 & 39 & 43 & - & - & 38 & 32 & 46 & 60 \\
\hline 786 & 45 & 40 & 43 & 39 & - & - & 41 & 36 & 42 & 55 \\
\hline 788 & 39 & 42 & 39 & 42 & - & - & 38 & 31 & 43 & 57 \\
\hline 791 & 42 & 38 & 43 & 39 & - & - & 41 & 36 & 42 & 57 \\
\hline 794 & 39 & 41 & 38 & 41 & - & - & 40 & 31 & 42 & 53 \\
\hline 797 & 43 & 37 & 41 & 38 & - & - & 41 & 36 & 43 & 57 \\
\hline 800 & 37 & 42 & 38 & 40 & - & - & 41 & 32 & 43 & 54 \\
\hline
\end{tabular}


CLOSED DOOR SHIELDED FIRE TEST

THERMOCOUPLES AND DISKS AT SPRINKLER AND DETECTOR LOCATION - ${ }^{\circ} \mathrm{C}$

\begin{tabular}{|c|c|c|c|c|c|c|c|c|c|c|}
\hline TIME & T24 & D25 & T26 & D27 & T28 & D29 & PAN A & PAN B & PAN C & PAN D \\
\hline 803 & 36 & 42 & 37 & 42 & - & - & 37 & 31 & 45 & 54 \\
\hline 806 & 36 & 41 & 36 & 41 & - & - & 38 & 31 & 44 & 52 \\
\hline 808 & 40 & 40 & 39 & 39 & - & - & 40 & 34 & 43 & 55 \\
\hline 811 & 36 & 40 & 38 & 39 & - & - & 40 & 33 & 42 & 56 \\
\hline 814 & 40 & 40 & 38 & 40 & - & - & 36 & 32 & 44 & 54 \\
\hline 817 & 41 & 37 & 41 & 37 & - & - & 39 & 35 & 43 & 55 \\
\hline 820 & 40 & 37 & 39 & 38 & - & -. & 36 & 34 & 44 & 58 \\
\hline 823 & 36 & 41 & 36 & 41 & - & - & 38 & 31 & 45 & 58 \\
\hline 826 & 36 & 41 & 35 & 41 & $=$ & - & 36 & 30 & 44 & 54 \\
\hline 829 & 41 & 36 & 40 & 36 & - & - & 39 & 35 & 43 & 56 \\
\hline 832 & 38 & 39 & 38 & 38 & $\longrightarrow$ & - & 40 & 34 & 41 & 56 \\
\hline 834 & 36 & 40 & 36 & 40 & - & - & 37 & 31 & 41 & 52 \\
\hline 837 & 35 & 40 & 36 & 40 & - & - & 35 & 30 & 41 & 55 \\
\hline 840 & 36 & 40 & 35 & 40 & - & - & 37 & 30 & 42 & 51 \\
\hline 843 & 37 & 38 & 38 & 38 & - & - & 39 & 32 & 40 & 51 \\
\hline 846 & 39 & 36 & 39 & 36 & - & - & 40 & 35 & 39 & 55 \\
\hline 849 & 41 & 37 & 40 & 36 & - & - & 39 & 33 & 39 & 52 \\
\hline 852 & 35 & 40 & 35 & 40 & - & - & 34 & 30 & 41 & 53 \\
\hline 854 & 41 & 36 & 40 & 36 & - & - & 38 & 34 & 39 & 54 \\
\hline 857 & 40 & 36 & 40 & 35 & - & - & 38 & 34 & 40 & 53 \\
\hline 860 & 41 & 36 & 39 & 36 & - & - & 36 & 34 & 41 & 53 \\
\hline 863 & 39 & 37 & 38 & 37. & - & - & 35 & 33 & 43 & 52 \\
\hline 866 & 40 & 36 & 39 & 36 & - & - & 36 & 34 & 41 & 52 \\
\hline 869 & 40 & 36 & 39 & 35 & - & - & 38 & 35 & 40 & 50 \\
\hline 872 & 39 & 36 & 39 & 35 & - & $\longrightarrow$ & 38 & 35 & 40 & 50 \\
\hline
\end{tabular}


THERMOCOUPLES AND DISKS AT SPRINKLER AND DETECTOR LOCATION - ${ }^{\circ} \mathrm{C}$

\begin{tabular}{|c|c|c|c|c|c|c|c|c|c|c|}
\hline TIME & T24 & D25 & T26 & $\mathrm{D} 27$ & $T 28$ & D29 & PAN A & PAN B & PAN C & PAN D \\
\hline 875 & 36 & 40 & 35 & 39 & - & - & 37 & 31 & 40 & 48 \\
\hline 877 & 39 & 35 & 38 & 35 & - & - & 39 & 35 & 40 & 50 \\
\hline 880 & 35 & 38 & 36 & 37 & - & - & 38 & 33 & 40 & 48 \\
\hline 883 & 34 & 39 & 34 & 39 & - & - & 35 & 31 & 41 & 47 \\
\hline 886 & 40 & 35 & 39 & 35 & - & $\ldots$ & 39 & 36 & 39 & 47 \\
\hline 889 & 34 & 40 & 33 & 39 & --. & - & 35 & 31 & 41 & 45 \\
\hline 892 & 34 & 39 & 34 & 38 & - & - & 36 & 32 & 42 & 44 \\
\hline 895 & 38 & 35 & 38 & 34 & - & - & 39 & 36 & 40 & 45 \\
\hline 898 & 34 & 39 & 34 & 39 & - & - & 35 & 31 & 42 & 45 \\
\hline 901 & 33 & 39 & 34 & 39 & - & - & 35 & 31 & 42 & 45 \\
\hline 903 & 38 & 35 & 37 & 35 & - & - & 35 & 34 & 42 & 46 \\
\hline 906 & 38 & 34 & 38 & 34 & - & - & 36 & 34 & 41 & 46 \\
\hline 909 & 37 & 34 & 36 & 34 & - & - & 35 & 34 & 41 & 44 \\
\hline 912 & 37 & 35 & 36 & 35 & - & - & 35 & 33 & 41 & 44 \\
\hline 915 & 38 & 33 & 37 & 34 & - & - & 36 & 34 & 41 & 44 \\
\hline 918 & 39 & 33 & 38 & 33 & - & - & 37 & 34 & 40 & 44 \\
\hline 921 & 38 & 34 & 37 & 34 & - & - & 36 & 34 & 40 & 46 \\
\hline 924 & 37 & 34 & 37 & 34 & - & - & 34 & 33 & 41 & 45 \\
\hline 926 & 38 & 33 & 38 & 33 & - & - & 36 & 34 & 40 & 46 \\
\hline 929 & 38 & 33 & 37 & 33 & - & - & 36 & 34 & 39 & 44 \\
\hline 932 & 33 & 37 & 34 & 37 & - & - & 36 & 30 & 38 & 43 \\
\hline 935 & 33 & 38 & 32 & 37 & - & - & 34 & 30 & 38 & 43 \\
\hline 938 & 36 & 33 & 36 & 33 & - & - & 37 & 33 & 37 & 42 \\
\hline 941 & 32 & 37 & 32 & 37 & - & - & 34 & 30 & 40 & 43 \\
\hline 944 & 32 & 37 & 33 & 36 & - & - & 33 & 30 & 40 & 43 \\
\hline
\end{tabular}


CLOSED DOOR SHIELDED FIRE TEST

THERMOCOUPLES AND DISKS AT SPRINKLER AND DETECTOR LOCATION - ${ }^{\circ} \mathrm{C}$

\begin{tabular}{|c|c|c|c|c|c|c|c|c|c|c|}
\hline TIME & T24 & $\mathrm{D} 25$ & T26 & $\mathrm{D} 27$ & T28 & $\mathrm{D} 29$ & PAN A & PAN B & PAN C & PAN D \\
\hline 947 & 33 & 36 & 33 & 36 & $\cdots$ & $\cdots$ & 32 & 29 & 41 & 44 \\
\hline 950 & 38 & 33 & 37 & 32 & - & - & 37 & 33 & 38 & 44 \\
\hline 953 & 35 & 34 & 35 & 34 & - & $\cdots$ & 33 & 31 & 38 & 44 \\
\hline 956 & 31 & 37 & 32 & 37 & 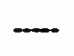 & - & 32 & 29 & 39 & 42 \\
\hline 959 & 32 & 37 & 32 & 37 & $\ldots$ & $\ldots$ & 33 & 29 & 39 & 42 \\
\hline 961 & 36 & 32 & 36 & 33 & - & - & 34 & 32 & 38 & 44 \\
\hline 964 & 31 & 37 & 32 & 37 & - & - & 33 & 28 & 39 & 42 \\
\hline 967 & 36 & 33 & 35 & 33 & $\cdots$ & - & 33 & 32 & 39 & 41 \\
\hline 970 & 37 & 32 & 36 & 31 & - & - & 36 & 34 & 38 & 43 \\
\hline 973 & 37 & 32 & 36 & 32 & - & - & 36 & 34 & 38 & 42 \\
\hline 976 & 36 & 31 & 36 & 32 & $\cdots$ & -- & 36 & 34 & 37 & 43 \\
\hline 979 & 37 & 32 & 36 & 32 & - & - & 34 & 33 & 37 & 43 \\
\hline 981 & 31 & 35 & 32 & 35 & $\cdots$ & -- & 35 & 30 & 37 & 41 \\
\hline 984 & 31 & 36 & 31 & 36 & - & - & 33 & 29 & 38 & 41 \\
\hline 987 & 35 & 31 & 36 & 31 & - & - & 36 & 33 & 36 & 43 \\
\hline 990 & 32 & 34 & 33 & 34 & - & - & 35 & 29 & 36 & 42 \\
\hline 993 & 31 & 36 & 31 & 36 & - & - & 32 & 28 & 38 & 42 \\
\hline 996 & 33 & 34 & 33 & 34 & - & - & 36 & 31 & 36 & 43 \\
\hline 999 & 32 & 36 & 31 & 36 & - & - & 31 & 28 & 37 & 42 \\
\hline 1002 & 31 & 36 & 31 & 36 & $-\infty$ & $\cdots$ & 32 & 28 & 38 & 42 \\
\hline 1004 & 36 & 32 & 35 & 32 & $\longrightarrow$ & - & 33 & 33 & 38 & 44 \\
\hline 1007 & 31 & 36 & 31 & 36 & $\cdots$ & - & 32 & 28 & 38 & 42 \\
\hline 1010 & 31 & 35 & 32 & 35 & - & - & 32 & 29 & 39 & 42 \\
\hline 1013 & 31 & 36 & 31 & 36 & - & - & 32 & 29 & 39 & 41 \\
\hline 1016 & 30 & 36 & 31 & 36 & $\longrightarrow$ & - & 32 & 28 & 39 & 41 \\
\hline
\end{tabular}


CLOSED DOOR SHIELDED FIRE TEST

THERMOCOUPLES AND DISKS AT SPRINKLER AND DETECTOR LOCATION - ${ }^{\circ} \mathrm{C}$

\begin{tabular}{|c|c|c|c|c|c|c|c|c|c|c|}
\hline TME & T24 & $\mathrm{D} 25$ & T26 & $\mathrm{D} 27$ & T28 & D29 & PAN A & PAN $B$ & PAN C & PAN D \\
\hline 1019 & 31 & 35 & 31 & 35 & - & - & 31 & 28 & 38 & 41 \\
\hline 1022 & 36 & 31 & 36 & 31 & - & - & 35 & 33 & 36 & 42 \\
\hline 1025 & 32 & 34 & 33 & 34 & - & - & 32 & 29 & 38 & 42 \\
\hline 1027 & 31 & 35 & 31 & 35 & - & - & 31 & 28 & 38 & 40 \\
\hline 1030 & 36 & 32 & 35 & 31 & - & - & 36 & 33 & 34 & 43 \\
\hline 1033 & 30 & 36 & 31 & 36 & - & - & 32 & 29 & 36 & 40 \\
\hline 1036 & 31 & 36 & 31 & 35 & - & - & 33 & 29 & 37 & 40 \\
\hline 1039 & 31 & 35 & 32 & 34 & - & - & 34 & 29 & 36 & 41 \\
\hline 1042 & 30 & 36 & 31 & 36 & - & - & 32 & 28 & 37 & 40 \\
\hline 1045 & 31 & 36 & 31 & 35 & - & - & 32 & 28 & 36 & 41 \\
\hline 1048 & 31 & 35 & 32 & 35 & - & - & 33 & 29 & 36 & 40 \\
\hline 1050 & 31 & 34 & 31 & 35 & - & - & 33 & 29 & 35 & 41 \\
\hline 1053 & 36 & 31 & 36 & 31 & - & - & 35 & 33 & 34 & 42 \\
\hline 1056 & 31 & 36 & 31 & 35 & - & - & 31 & 29 & 36 & 41 \\
\hline 1059 & 31 & 36 & 31 & 36 & - & - & 31 & 28 & 37 & 40 \\
\hline 1062 & 31 & 36 & 31 & 36 & - & - & 31 & 28 & 36 & 40 \\
\hline 1065 & 30 & 36 & 31 & 35 & - & - & 31 & 28 & 37 & 40 \\
\hline 1068 & 34 & 31 & 34 & 31 & - & - & 32 & 32 & 36 & 41 \\
\hline 1071 & 35 & 32 & 34 & 33 & - & - & 31 & 31 & 36 & 41 \\
\hline 1073 & 30 & 36 & 31 & 35 & - & - & 32 & 28 & 37 & 43 \\
\hline 1076 & 35 & 31 & 34 & 31 & - & - & 32 & 32 & 36 & 45 \\
\hline 1079 & 33 & 34 & 33 & 34 & - & - & 31 & 29 & 37 & 43 \\
\hline 1082 & 36 & 33 & 34 & 32 & - & - & 32 & 31 & 36 & 43 \\
\hline 1085 & 35 & 31 & 35 & 31 & - & - & 35 & 33 & 35 & 41 \\
\hline 1088 & 35 & 31 & 35 & 31 & - & - & 35 & 33 & 34 & 43 \\
\hline
\end{tabular}


CLOSED DOOR SHIELDED FIRE TEST

THERMOCOUPLES AND DISKS AT SPRINKLER AND DETECTOR LOCATION - ${ }^{\circ} \mathrm{C}$

\begin{tabular}{|c|c|c|c|c|c|c|c|c|c|c|}
\hline TIME & T24 & D25 & T26 & D27 & T28 & $\mathrm{D} 29$ & PAN A & PAN B & PAN C & PAN D \\
\hline 1091 & 31 & 34 & 32 & 34 & - & - & 34 & 30 & 34 & 42 \\
\hline 1094 & 31 & 35 & 31 & 35 & - & - & 31 & 28 & 36 & 42 \\
\hline 1096 & 34 & 33 & 34 & 32 & - & - & 35 & 33 & 34 & 42 \\
\hline 1099 & 34 & 32 & 34 & 31 & - & $\cdots$ & 35 & 32 & 34 & 44 \\
\hline 1102 & 36 & 31 & 34 & 31 & - & ---- & 34 & 32 & 34 & 44 \\
\hline 1105 & 32 & 34 & 33 & 33 & - & - & 35 & 31 & 34 & 41 \\
\hline 1108 & 32 & 32 & 33 & 31 & - & - & 35 & 32 & 35 & 42 \\
\hline 1111 & 34 & 32 & 34 & 31 & $\cdots$ & - & 35 & 32 & 35 & 43 \\
\hline 1114 & 31 & 35 & 31 & 35 & - & $\cdots$ & 33 & 29 & 36 & 45 \\
\hline 1117 & 31 & 36 & 31 & 36 & - & - & 31 & 28 & 37 & 45 \\
\hline 1119 & 31 & 36 & 31 & 35 & - & - & 32 & 28 & 37 & 47 \\
\hline 1122 & 36 & 32 & 35 & 32 & - & $\cdots$ & 36 & 32 & 35 & 45 \\
\hline 1125 & 35 & 33 & 34 & 33 & - & $\cdots$ & 32 & 32 & 36 & 45 \\
\hline 1128 & 32 & 36 & 31 & 36 & - & - & 31 & 28 & 36 & 44 \\
\hline 1131 & 36 & 32 & 35 & 32 & - & - & 35 & 32 & 34 & 47 \\
\hline 1134 & 37 & 33 & 35 & 32 & - & - & 32 & 32 & 36 & 47 \\
\hline 1137 & 32 & 36 & 31 & 36 & - & - & 31 & 28 & 36 & 45 \\
\hline 1140 & 36 & 31 & 36 & 31 & - & $\cdots$ & 35 & 32 & 35 & 46 \\
\hline 1142 & 35 & 33 & 34 & 33 & - & - & 32 & 30 & 37 & 45 \\
\hline 1145 & 36 & 32 & 35 & 31 & - & - & 32 & 33 & 37 & 44 \\
\hline 1148 & 36 & 32 & 35 & 31 & - & - & 35 & 33 & 35 & 44 \\
\hline 1151 & 32 & 35 & 32 & 35 & - & - & 32 & 29 & 37 & 42 \\
\hline 1154 & 36 & 32 & 36 & 31 & - & - & 36 & 33 & 34 & 44 \\
\hline 1157 & 36 & 32 & 35 & 31 & - & - & 36 & 33 & 34 & 44 \\
\hline 1160 & 31 & 36 & 31 & 36 & - & - & 31 & 28 & 36 & 43 \\
\hline
\end{tabular}


CLOSED DOOR SHIELDED FIRE TEST

THERMOCOUPLES AND DISKS AT SPRINKLER AND DETECTOR LOCATION - ${ }^{\circ} \mathrm{C}$

\begin{tabular}{|c|c|c|c|c|c|c|c|c|c|c|}
\hline TME & $T 24$ & $\mathrm{D} 25$ & T26 & 027 & T28 & $\mathrm{D} 29$ & PAN A & PAN B & PAN C & PAN D \\
\hline 1163 & 33 & 35 & 33 & 35 & - & - & 35 & 31 & 35 & 46 \\
\hline 1165. & 36 & 32 & 35 & 32 & - & - & 36 & 32 & 34 & 47 \\
\hline 1168 & 32 & 36 & 32 & 36 & - & - & 31 & 28 & 37 & 45 \\
\hline 1171 & 37 & 33 & 35 & 32 & - & - & 36 & 32 & 34 & 46 \\
\hline 1174 & 31 & 37 & 31 & 36 & - & - & 32 & 28 & 36 & 43 \\
\hline 1177 & 36 & 33 & 35 & 32 & - & - & 33 & 33 & 36 & 50 \\
\hline 1180 & 37 & 32 & 36 & 31 & - & - & 36 & 33 & 34 & 50 \\
\hline 1183 & 37 & 33 & 34 & 32 & $\ldots$ & - & 32 & 31 & 36 & 46 \\
\hline 1186 & 35 & 34 & 34 & 33 & - & - & 33 & 32 & 36 & 44 \\
\hline 1188 & 36 & 32 & 35 & 32 & - & - & 36 & 32 & 34 & 43 \\
\hline 1191 & 31 & 36 & 31 & 35 & - & - & 31 & 28 & 36 & 46 \\
\hline 1194 & 37 & 32 & 36 & 31 & - & - & 35 & 33 & 33 & 46 \\
\hline 1197 & 33 & 34 & 34 & 33 & - & - & 36 & 32 & 33 & 47 \\
\hline 1200 & 33 & 36 & 31 & 36 & - & - & 32 & 28 & 35 & 48 \\
\hline 1203 & 35 & 34 & 35 & 33 & - & - & 36 & 32 & 33 & 46 \\
\hline 1206 & 37 & 32 & 35 & 31 & - & - & 36 & 33 & 33 & 44 \\
\hline 1208 & 31 & 36 & 32 & 35 & - & - & 35 & 30 & 33 & 44 \\
\hline 1211 & 31 & 36 & 31 & 36 & - & - & 32 & 28 & 36 & 47 \\
\hline 1214 & 32 & 36 & 32 & 35 & - & - & 33 & 29 & 35 & 47 \\
\hline 1217 & 36 & 32 & 35 & 31 & - & - & 34 & 33 & 34 & 47 \\
\hline 1220 & 36 & 33 & 35 & 33 & - & - & 33 & 31 & 35 & 46 \\
\hline 1223 & 35 & 33 & 35 & 33 & - & - & 34 & 31 & 36 & 44 \\
\hline 1226 & 36 & 32 & 36 & 31 & - & - & 36 & 33 & 34 & 43 \\
\hline 1229 & 35 & 34 & 34 & 34 & - & - & 32 & 31 & 36 & 44 \\
\hline 1232 & 37 & 32 & 36 & 32 & - & - & 34 & 33 & 34 & 45 \\
\hline
\end{tabular}


CLOSED DOOR SHIELDED FIRE TEST

THERMOCOUPLES AND DISKS AT SPRINKLER AND DETECTOR LOCATION - ${ }^{\circ} \mathrm{C}$

\begin{tabular}{|c|c|c|c|c|c|c|c|c|c|c|}
\hline TIME & $T 24$ & $\mathrm{D} 25$ & T26 & $\mathrm{D} 27$ & $T 28$ & D29 & PAN A & PAN B & PAN C & PAN D \\
\hline 1234 & 34 & 33 & 35 & 32 & - & - & 36 & 32 & 33 & 44 \\
\hline 1237 & 34 & 33 & 34 & 32 & - & - & 36 & 32 & 33 & 46 \\
\hline 1240 & 37 & 33 & 35 & 32 & - & - & 36 & 33 & 33 & 46 \\
\hline 1243 & 36 & 33 & 35 & 32 & - & - & 36 & 32 & 33 & 48 \\
\hline 1246 & 31 & 37 & 31 & 36 & - & $\cdots$ & 33 & 28 & 35 & 43 \\
\hline 1249 & 35 & 34 & 34 & 33 & - & --- & 36 & 32 & 33 & 45 \\
\hline 1252 & 36 & 33 & 35 & 32 & --- & -- & 36 & 32 & 33 & 45 \\
\hline 1255 & 32 & 36 & 32 & 35 & - & - & 32 & 29 & 35 & 45 \\
\hline 1257 & 32 & 36 & 31 & 35 & - & - & 32 & 29 & 35 & 44 \\
\hline 1260 & 32 & 36 & 31 & 36 & - & - & 33 & 28 & 36 & 46 \\
\hline 1263 & 37 & 33 & 35 & 32 & - & - & 35 & 32 & 34 & 47 \\
\hline 1266 & 37 & 33 & 35 & 32 & - & - & 34 & 33 & 34 & 48 \\
\hline 1269 & 37 & 33 & 35 & 32 & - & - & 34 & 33 & 34 & 50 \\
\hline 1272 & 36 & 34 & 34 & 32 & - & $\cdots$ & 33 & 32 & 35 & 48 \\
\hline 1275 & 33 & 35 & 33 & 34 & - & - & 37 & 30 & 33 & 46 \\
\hline 1278 & 32 & 36 & 31 & 36 & - & - & 32 & 28 & 36 & 44 \\
\hline 1280 & 37 & 32 & 36 & 31 & - & - & 36 & 33 & 33 & 46 \\
\hline 1283 & 32 & 36 & 32 & 35 & - & - & 35 & 29 & 34 & 43 \\
\hline 1286 & 33 & 36 & 32 & 35 & - & - & 34 & 29 & 34 & 46 \\
\hline 1289 & 34 & 34 & 34 & 32 & - & - & 36 & 33 & 33 & 48 \\
\hline 1292 & 32 & 37 & 32 & 36 & - & - & 33 & 28 & 35 & 47 \\
\hline 1295 & 37 & 33 & 36 & 33 & - & - & 33 & 32 & 35 & 50 \\
\hline 1298 & 36 & 33 & 35 & 32 & - & - & 33 & 32 & 35 & 49 \\
\hline 1301 & 32 & 37 & 39 & 36 & - & - & 32 & 28 & 35 & 47 \\
\hline 1303 & 36 & 33 & 35 & 32 & - & - & 34 & 32 & 35 & 48 \\
\hline
\end{tabular}


CLOSED DOOR SHIELDED FIRE TEST

THERMOCOUPLES AND DISKS AT SPRINKLER AND DETECTOR LOCATION - ${ }^{\circ} \mathrm{C}$

\begin{tabular}{ccccccccccc}
\hline TIME & T24 & D25 & T26 & D27 & T28 & D29 & PAN A & PAN B & PAN C & PAN D \\
\hline 1306 & 37 & 32 & 36 & 31 & -3 & -3 & 33 & 33 & 33
\end{tabular}


NIST-114A

(REV. 3-90)

\section{U.S. DEPARTMENT OF COMMERCE \\ NATIONAL INSTITUTE OF STANDARDS AND TECHNOLOGY BIBLIOGRAPHIC DATA SHEET}

1. PUBUCATION OR AEPORT NUMBER NISTIR 5240

2 PERFOAMINO ORGANIZATION AEPOAT MUMBEA

3. PUBUCATION DATE

July 1993

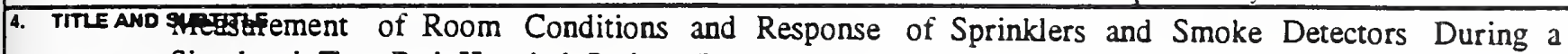

Simulated Two-Bed Hospital Patient Room Fire

5. AUTHOR(S)

Kathy A. Notarianni

6. PERFORMINO ORGANIZATION (IF JOINT OR OTHER THAN NIST, SEE INSTRUCTIONS)

U.S. DEPARTMENT OF COMMERCE

MATIONAL INSTITUTE OF STAMDARDS AND TECHNOLOCY

GATTHERSBURG, MD 20899

7. CONTRACT/ORANT NUMBER

8. TYPE OF REPORT AND PEAIOD COVERED

9. SPONSORINO ORGANIZATION MAME AND COMPLETE ADORESS (STREET, CITY, STATE, ZIP)

National Institutes of Health

Division of Engineering Services

Bethesda, MD 20892

10. SUPPLEMENTAAY NOTES

11. ABSTRACT (A 2OO-WORD OR LESS FACTUAL SUMMARY OF MOST SIGMIFICANT INFORMATION. IF DOCUMENT INCLUDES A SIGNIFICANT BIBUOGRAPHY OA UTERATURE SURVEY, MENTION IT HERE)

A series of experiments are reported in which a wood crib was burned within a simulated two-bed hospital patient room in order to measure the activation times of various types of quick and standard response sprinklers and ionization and photoelectric smoke detectors at several locations in the room simulating multiple options for protection of the space. Gas and surface temperatures, heat flux, carbon dioxide, carbon monoxide, and oxygen concentrations were continuously measured in order to access the tenability of the room. Of the parameters measured, temperature was the best indicator of the tenability of the space. Temperature at time of activation of the quick response sprinklers was at or below $77^{\circ} \mathrm{C}\left(171^{\circ} \mathrm{F}\right)$ at the 1.52 $\mathrm{m}\left(5 \mathrm{ft}\right.$.) level and at or below $48^{\circ} \mathrm{C}\left(118^{\circ} \mathrm{F}\right)$ at the $0.91 \mathrm{~m}(3 \mathrm{ft}$.) level between the patient beds, in all experiments conducted with the exception of the shielded fire scenario in which temperatures at the five and three foot levels reached $111^{\circ} \mathrm{C}(232$ $\left.{ }^{\circ} \mathrm{F}\right)$, and $78{ }^{\circ} \mathrm{C}\left(172{ }^{\circ} \mathrm{F}\right)$ respectively. The initial detector activation was received between 232-377 seconds prior to activation of the first sprinkler, and $552-722$ seconds prior to activation of the QR-EC sidewall sprinkler.

12. KEY WORDS (6 TO 12 ENTRIES; ALPHABETICAL ORDER; CAPTTALZE OMLY PAOPER NAMES; AND SEPARATE KEY WOADS BY SEMICOLONS) Sprinklers, fire tests, sprinkler response, smoke detectors, hospital fires, life safety 

